\title{
The Evolution of Distant Spiral Galaxies in the FORS Deep Field
}

\author{
Dissertation \\ zur Erlangung des Doktorgrades \\ der Mathematisch-Naturwissenschaftlichen Fakultäten \\ der Georg-August-Universität zu Göttingen
}

vorgelegt von

Asmus Böhm

aus Kassel

Göttingen 2003 
D7

Referent: Prof. Dr. K. J. Fricke

Korreferent: Prof. Dr. F. Kneer

Tag der mündlichen Prüfung: 5. Dez. 2003 


\section{Contents}

1 Introduction $\quad 7$

1.1 The Hierarchical Scenario . . . . . . . . . . . . . . . . . . . . 7

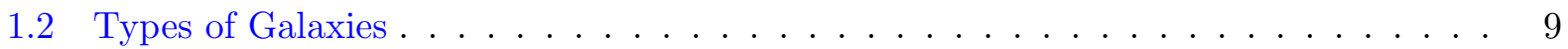

1.2 .1 Morphology . . . . . . . . . . . . . . . . . . . . 9 9

1.2 .2 Spectral Energy Distribution _. . . . . . . . . . . . . . . 10

1.2 .3 Kinematics . . . . . . . . . . . . . . . . . . 11

1.3 Scaling Relations . . . . . . . . . . . . . . . . . . . . . . . 12

1.4 Galaxy Evolution . . . . . . . . . . . . . . . . . . . . 14

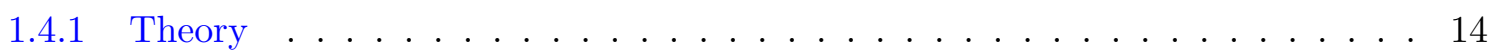

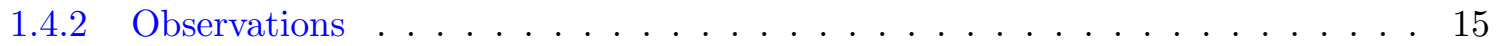

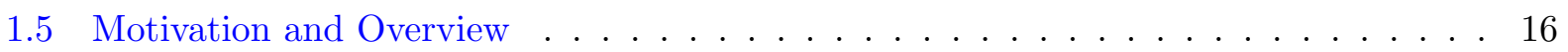

2 Construction of the Sample $\quad 19$

2.1 The FORS Deep Field . . . . . . . . . . . . . . . . . . . . . . . . 19

2.2 Instrument Configuration . . . . . . . . . . . . . . . . . 20

2.3 Basic Selection Criteria . . . . . . . . . . . . . . . . . . 21

2.3 .1 Apparent Brightness . . . . . . . . . . . . . . . . . . . 22

2.3.2 Spectrophotometric Type . . . . . . . . . . . . . . . . . . . . . . . . . . . . . 22

2.3.3 Structural Parameters . . . . . . . . . . . . . . . . . . . . . . . . . . . . . . . 22

2.3.4 Photometric Redshift . . . . . . . . . . . . . . . . . 23

2.4 1999: A Pilot Observation . . . . . . . . . . . . . . . . . 23

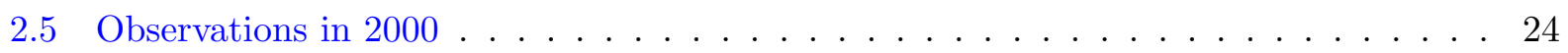

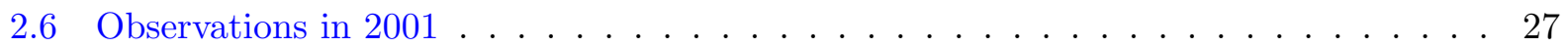

3 Data Reduction $\quad 31$

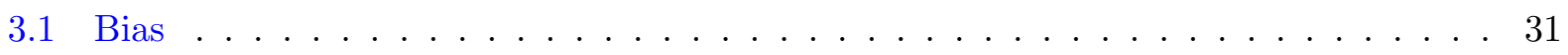

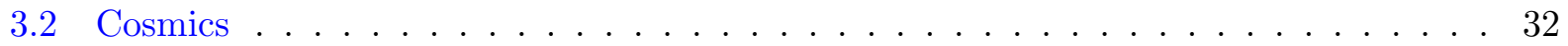

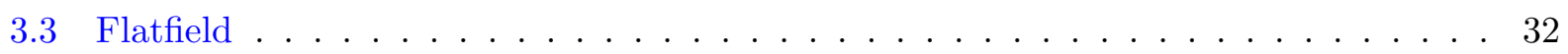

3.4 Rectification . . . . . . . . . . . . . . . . 33

3.5 Wavelength Calibration . . . . . . . . . . . . . . . 33

3.6 Sky Subtraction . . . . . . . . . . . . . . . . . . . . . . . . . . . . . . . . . . .

3.7 I-band Reference Frame . . . . . . . . . . . . . . . . . . . 36 
4 Spectrophotometric Analysis $\quad 39$

4.1 Redshift Distribution . . . . . . . . . . . . . . . . . . . . . . 39

4.2 Spectral Classification . . . . . . . . . . . . . . . . . . 42

4.3 Surface Brightness Profile Fitting . . . . . . . . . . . . . . . . . . . . 43

4.4 An HDF Simulation . . . . . . . . . . . . . . . . . . . . 46

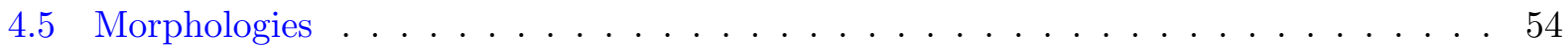

4.6 Luminosity Derivation . . . . . . . . . . . . . . . . . 60

4.6.1 Apparent Magnitudes . . . . . . . . . . . . . . . . 60 60

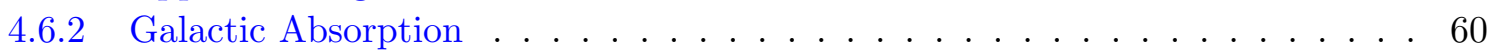

4.6 .3 Distance Modulus . . . . . . . . . . . . . . . . . . . 6 61 . . . . . . . .

4.6 .4 K-Correction . . . . . . . . . . . . . . . . 61

4.6 .5 Intrinsic Absorption . . . . . . . . . . . . . . . . . . 64

4.7 Luminosity Distribution and Errors . . . . . . . . . . . . . . . 66

$\begin{array}{lll}5 & \text { Kinematic Analysis } & 69\end{array}$

5.1 Rotation Curve Extraction . . . . . . . . . . . . . . . . 70

5.2 Simulated Rotation Curves . . . . . . . . . . . . . . . . . . 71

5.2 .1 The Intrinsic Shape . . . . . . . . . . . . . . . . . . . . 71

5.2 .2 The Modelling . . . . . . . . . . . . . . . . . . 73

5.2 .3 Error Budget . . . . . . . . . . . . . . . . . 78

5.2.4 Comparison between Different Emission Lines . . . . . . . . . . . . . . . 80

5.2.5 Comparison between Different Intrinsic RC Shapes . . . . . . . . . . . . . . 82

5.3 Distribution in $\mathrm{V}_{\max } \ldots \ldots \ldots \ldots \ldots \ldots \ldots \ldots \ldots$

5.4 Total Masses . . . . . . . . . . . . . . . . . . . . . . 85

6 Scaling Relations at Intermediate Redshift $\quad \mathbf{8 7}$

6.1 The Local Reference . . . . . . . . . . . . . . . . . . . . . . . 87

6.2 The Distant Tully-Fisher Relation . . . . . . . . . . . . . . . . . 89

6.3 The Distant Velocity-Size Relation . . . . . . . . . . . . . . . . . . 93

6.4 Discussion . . . . . . . . . . . . . . . . . . . . . . 95

6.4 .1 Test of Incompleteness . . . . . . . . . . . . . . . . . . 95

6.4.2 An Effect of the Color-Residual Relation? . . . . . . . . . . . . . . . . . 99

6.4.3 Test of Environmental Effects . . . . . . . . . . . . . . . . . 101

6.4.4 Star Formation Rates . . . . . . . . . . . . . . . . . . . 103

6.4.5 The Intrinsic RC Shape . . . . . . . . . . . . . . . . . . . . . 104

6.4.6 Intrinsic Absorption Correction . . . . . . . . . . . . . . . . . . . . 104

6.4.7 The Influence of Ground-Based Imaging . . . . . . . . . . . . . . . . . . . 105

6.5 Previous Studies . . . . . . . . . . . . . . . . . . . 105

6.6 Stellar Population Models . . . . . . . . . . . . . . . . . . . 107

6.7 Further Discussion and Conclusions . . . . . . . . . . . . . 107

7 Summary and Outlook $\quad 111$

$\begin{array}{ll}\text { A Spectrophotometric Data } & 115\end{array}$ 
$\begin{array}{lr}\text { B Kinematic Data } & 123\end{array}$

$\begin{array}{ll}\text { C Data on the Scaling Relations } & 125\end{array}$

$\begin{array}{lr}\text { References } & 127\end{array}$ 



\section{Deutsche Zusammenfassung}

Dieser Abschnitt verfolgt das Ziel, dem Leser das Verständnis der grundsätzlichen Fragestellungen, angewendeten Methoden und Schlussfolgerungen $\mathrm{zu}$ erleichtern, die in den folgenden Kapiteln ausführlich dargestellt werden. Die in Kapitel 7 gegebene Zusammenfassung setzt Kenntnisse in extragalaktischer Astrophysik voraus.

In den vergangenen Jahren hat eine Vielzahl von Beobachtungen, sowohl mit bodengebundenen als auch weltraumgestützten Teleskopen, in Kombination mit den Ergebnissen numerischer Simulationen, ein detailliertes Bild unseres Kosmos ergeben. Wichtige Parameter (wie z.B. das Weltalter), die noch vor rund 10 Jahren mit großen Unsicherheiten behaftet waren, konnten durch jüngste Untersuchungen z.T. sehr genau bestimmt werden.

Danach ist das heute beobachtbare Universum vor rund 14 Gigajahren aus einem dichten, heißen Urknall hervorgegangen. Der Kosmos hat auf großen räumlichen Skalen eine flache Metrik, d.h., die vierdimensionale Raumzeit ist weder hyperbolisch noch sphärisch gekrümmt. Die Expansionsrate des Universums nimmt jedoch nicht, wie ursprünglich angenommen, mit der Zeit ab, sondern zu, wobei die gegenwärtige Relativgeschwindigkeit entfernter Galaxien als Funktion ihrer Entfernung einer Hubble-Konstanten von $H_{0}=70 \mathrm{~km} \mathrm{~s}^{-1} \mathrm{Mpc}^{-1}$ entspricht $^{1}$. Die mittlere Dichte setzt sich nur zu $3 \%$ aus baryonischer Materie und zu rund $30 \%$ aus Dunkler Materie (DM) zusammen, der Hauptbeitrag von knapp 70\% rührt von der sog. Dunklen Energie her. Zwar ist bisher weder die Natur der DM (die rein gravitativ in Erscheinung tritt), noch jene der Dunklen Energie geklärt, die empirische Evidenz für ihre Existenz ist jedoch sehr hoch.

\footnotetext{
${ }^{1}$ Ein parsec (pc) entspricht einer Entfernung von rund 3.3 Lichtjahren oder $3.1 \cdot 10^{16} \mathrm{~m}$.
}

Aus dem Vorhandensein einer DM-Komponente können wichtige Schlussfolgerungen in Bezug auf die Strukturbildung im frühen Universum und deren Entwicklung mit fortschreitendem Weltalter gezogen werden. Kleinere Strukturen haben sich demnach aus primordialen Dichtefluktuationen als erste gebildet und im weiteren Zeitverlauf durch gravitative Wechselwirkung sukzessive größere Strukturen aufgebaut. Aus diesem hierarchischen Szenario lassen sich mit $N$-Körper-Simulationen Vorhersagen über die Entstehung und Entwicklung von einzelnen Galaxien und von größeren Systemen, wie Galaxienhaufen und Superhaufen, treffen.

Für das Verstehen der großräumigen Struktur auf Skalen $>1 \mathrm{Mpc}$, die im heutigen Kosmos beobachtet wird, hat sich das hierarchische Modell als sehr erfolgreich erwiesen. Auf der Skala einzelner Galaxien $(\sim 10 \mathrm{kpc})$ wurden hingegen teilweise Widersprüche zwischen theoretischen Vorhersagen und Beobachtungsergebnissen gefunden. So ist beispielsweise die in der Umgebung der Milchstraße beobachtete Anzahl der Galaxien sehr geringer Masse um Größenordnungen kleiner, als es sich in Simulationen ergibt. Auch weisen massereiche, lokale (d.h., heutige) Spiralgalaxien in N-Körper-Simulationen blauere Farben auf als massearme, während Beobachtungen das Gegenteil zeigen.

Das Verständnis der fundamentalen Prozesse, die bei der Galaxienentwicklung eine Rolle spielen — wobei sich der Begriff Entwicklung auf die zeitliche Änderung von verschiedenen Parametern wie Größe, Masse, Leuchtkraft, Spektraltyp, Metallgehalt etc. beziehen kann —, läßt sich insbesondere durch die Beobachtung von Galaxien vertiefen, die weit entfernt sind. Bedingt durch die lange Lichtlaufzeit blickt man dabei in frühere Epochen des Universums zurück. So entspricht z.B. eine kosmologische DopplerRotverschiebung von $z=0.5$ einer Rückblickzeit von rund 5 Gigajahren und repräsentiert damit ein Weltalter von nur rund $65 \%$ des heutigen Wertes. 
In den letzten Jahren haben sich verschiedene Studien mit der Untersuchung von Spiralgalaxien in solchen Entfernungen befasst, jedoch sind die dabei gefundenen Ergebnisse widersprüchlich. Insbesondere wurden Leuchtkraftentwicklungen für den Zeitraum der letzten Gigajahre abgeleitet, die um bis zu einem Faktor 6 voneinander abweichen! Ein zentrales Problem dieser Untersuchungen war dabei die geringe Größe der Datensätze von nur 10-20 Galaxien.

Das Ziel der vorliegenden Arbeit war es, Spektren und Direktbilder eines großen Samples von rund 100 Spiralgalaxien im Rotverschiebungsbereich $0.1 \leq z \leq 1.0$ zu gewinnen und deren Entwicklung z.B. in Leuchtkraft und Größe zu bestimmen. Mit diesem Datensatz, der mehr als das halbe Weltalter überstreicht, sollten die Vorhersagen des hierarchischen Szenarios quantitativ getestet und Gründe für die Diskrepanzen zwischen früheren Studien gefunden werden.

Ein wichtiges methodisches Hilfsmittel für diese Arbeit sind die sog. Skalenrelationen, durch die bestimmte Parameter von Galaxien miteinander korreliert sind. Das bekannteste Beispiel ist die Tully-Fisher-Relation (TFR), in der ein Zusammenhang zwischen der Leuchtkraft $L$ und der maximalen Rotationsgeschwindigkeit $V_{\max }$ (Erläuterung siehe unten) von Spiralgalaxien beobachtet wird. Die TFR bildet das zentrale Instrument in der vorliegenden Studie und soll daher im Folgenden eingehend erklärt werden.

Spiralgalaxien sind durch Rotation stabilisiert, d.h., Eigengravitation und Zentrifugalkräfte befinden sich im Gleichgewicht. Beobachtet man eine Spektrallinie im Spektrum einer Spiralgalaxie als Funktion des projizierten Abstandes von der Rotationsachse, stellt man daher $\mathrm{zu}$ einer Seite der Galaxienscheibe hin eine DopplerRotverschiebung, zur anderen Seite hin eine Blauverschiebung fest. Aus diesen Verschiebungen kann man die Rotationsgeschwindigkeit bestimmen (siehe Abb. 1).

Die Scheiben von Spiralgalaxien zeigen einen als Funktion des Radius exponentiell abfallenden

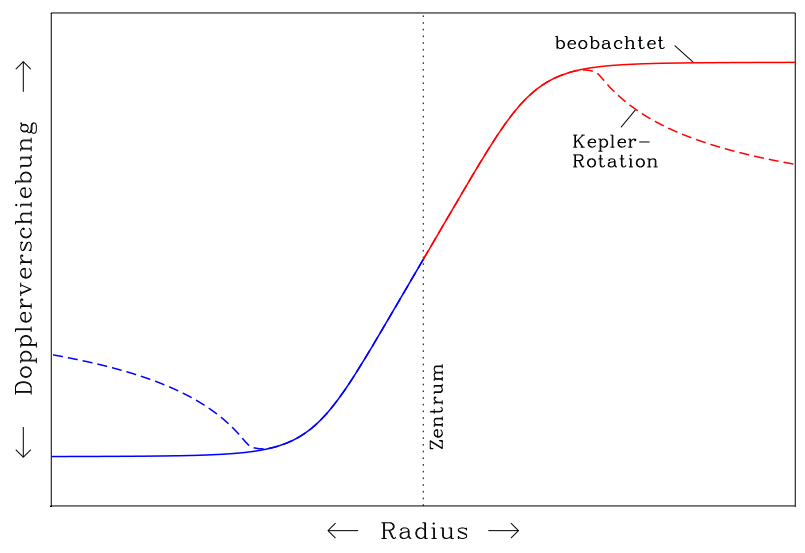

Abbildung 1: Schema der Rotationskurve einer Spiralgalaxie. Skizziert ist die Rot- bzw. Blauverschiebung einer Spektrallinie als Funktion des projizierten Abstandes von der Rotationsachse (gepunktete Linie). Zu großen Radien hin wäre für ein exponentiell abfallendes Massendichteprofil ein Abfall der Rotationsgeschwindigkeit mit $\sqrt{1 / r}$ zu erwarten (Kepler-Rotation, gestrichelte Linie), wohingegen sich in den Beobachtungen fast immer ein konstanter Wert zeigt, die maximale Rotationsgeschwindigkeit $V_{\max }$.

Helligkeitsverlauf $I \propto \mathrm{e}^{-r / r_{\mathrm{d}}}$; hierbei ist $r_{\mathrm{d}}$ ein Maß für die Scheibengröße, genannt Skalenlänge. Es läßt sich zeigen, daß für einen analogen Verlauf des Massendichteprofils die Rotationsgeschwindigkeit für Radien $0 \geq r \geq 2.2 r_{\mathrm{d}}$ linear auf ein Maximum ansteigen und im Bereich $r>2.2 r_{\mathrm{d}}$ mit $\sqrt{1 / r}$ abfallen müsste (KeplerRotation). Beobachtet wird jedoch in den meisten Fällen ein konstanter Wert $V_{\max }$, die maximale Rotationsgeschwindigkeit. Hieraus schließt man auf eine Komponente aus DM, die für große Radien die Massenverteilung dominiert und deren Masse linear mit dem Radius wächst. Mit numerischen Simulationen konnte gezeigt werden, daß die Gesamtmasse oder Virialmasse $M_{\text {vir }}$ einer Spiralgalaxie approximiert werden kann gemäß

$$
M_{\mathrm{vir}} \propto r_{\mathrm{d}} V_{\max }^{2}
$$

d.h., $V_{\max }$ kann als Massen-Indikator verwendet werden. 
Die TFR, also die Korrelation von $L$ und $V_{\max }$, lässt sich verstehen als eine Folge des Virialsatzes und der Stabilisation der Scheiben von Spiralgalaxien durch Rotation: Je massereicher eine Galaxie, desto schneller rotiert sie im zentrifugalen Gleichgewicht, und desto mehr stellare Masse enthält sie und ist daher leuchtkräftiger. Die Bedeutung der TFR für die Untersuchung der Entwicklung von Galaxien liegt nun darin, daß sich durch $N$-Körper-Simulationen auf der Basis des hierarchischen Szenarios Vorhersagen über die Form der TFR im früheren Universum machen lassen.

In einigen Simulationen wurde eine Entwicklung des Nullpunkts der TFR mit der Rückblickzeit gefunden, siehe Abb. 2. Dies entspricht einer Leuchtkraft von weit entfernten Spiralgalaxien, die bei gegebenem $V_{\max }$ (gegebener Masse) höher ist als bei Spiralen im lokalen Universum, bedingt durch eine jüngere Sternpopulation und dadurch einen höheren Anreil an massereichen, heißen Sternen mit kurzer Lebensdauer. Der Einfluß der jüngeren Sternpopulation wird dabei teilweise durch simultan ablaufende Prozesse kompensiert. So sind - dem Szenario einer hierarchischen Strukturbildung entsprechend - für ein gegebenes $V_{\max }$ die Scheiben weit entfernter Spiralen kleiner als im lokalen Universum, zudem ist der Gasanteil höher, da weniger Zeit für die Sternentstehung zur Verfügung stand. Das Gas trägt jedoch nur unwesentlich zur Leuchtkraft bei.

Eine konzeptionelle Schwierigkeit von $N$-KörperSimulationen besteht allerdings in ihrer geringen Auflösung. Bei der Modellierung von Galaxien haben die einzelnen Teilchen typischerweise rund $10^{6}$ Sonnenmassen. Für eine Berücksichtigung von Sternbildungsprozessen, Supernovae etc. müssen daher vereinfachende Parametrisierungen in die Rechnungen eingearbeitet werden.

Aus Beobachtungen geht hervor, daß manche Merkmale der Sternpopulationen in Galaxien von deren Masse abhängen. So zeigen z.B. massereiche Spiralgalaxien rötere Farben als

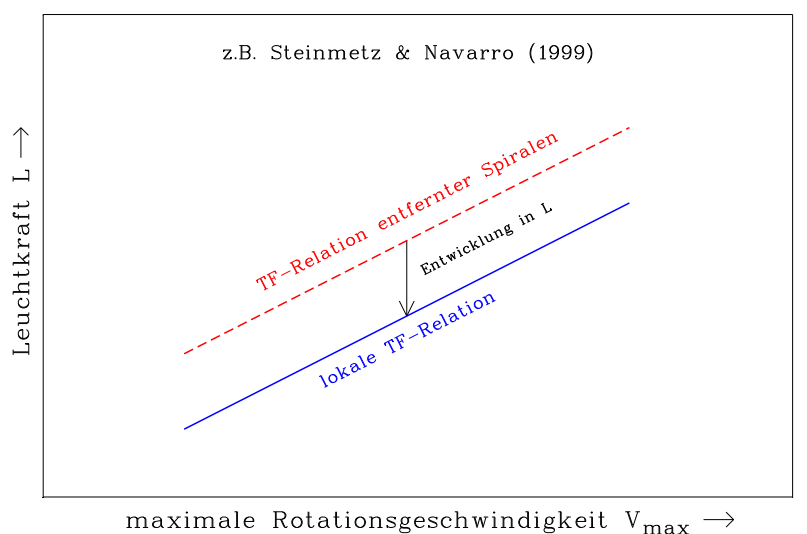

Abbildung 2: Entwicklung der Tully-Fisher-Relation, die in einigen $N$-Körper-Simulationen gefunden wurde. Weit entfernte Spiralgalaxien haben danach eine höhere Leuchtkraft als im lokalen Universum, dies bedingt einen Unterschied der Nullpunkte von entfernter und lokaler Tully-Fisher-Relation. Der Pfeil deutet die vorhergesagte Abnahme der Leuchtkraft für ein gegebenes $V_{\max }$ mit fortschreitendem Weltalter an. Nähere Erläuterungen siehe Text.

massearme. Dieser Zusammenhang konnte bislang noch nicht befriedigend aus den fundamentalen Randbedingungen von $N$-KörperSimulationen abgeleitet werden. Stattdessen war es notwendig, die Simulationen mit den spektrochemischen Eigenschaften heutiger Galaxien zu kalibrieren.

Einige Modelle, in denen die beobachtete Massenabhängigkeit der Farben oder des Metallgehalts (Häufigkeit der Elemente mit Ordnungszahlen $\geq 3$ ) parametrisiert wurde, sagen eine Änderung der Steigung der TFR mit wachsender Rückblickzeit voraus (siehe Abb. 3). Massearme, entfernte Spiralgalaxien wären demnach leuchtschwächer und massereiche, entfernte Spiralen leuchtkräftiger als im lokalen Universum. Dies lässt sich so verstehen, daß der bei entfernten Spiralgalaxien höhere Gasanteil und die geringere Scheibengröße sich bei massearmen Spiralgalaxien stärker auswirken als die jüngere Sternpopulation. Bei schnell rotierenden, also massereichen Spiralen ist die Situation umgekehrt, hier dominiert das geringere mittlere 


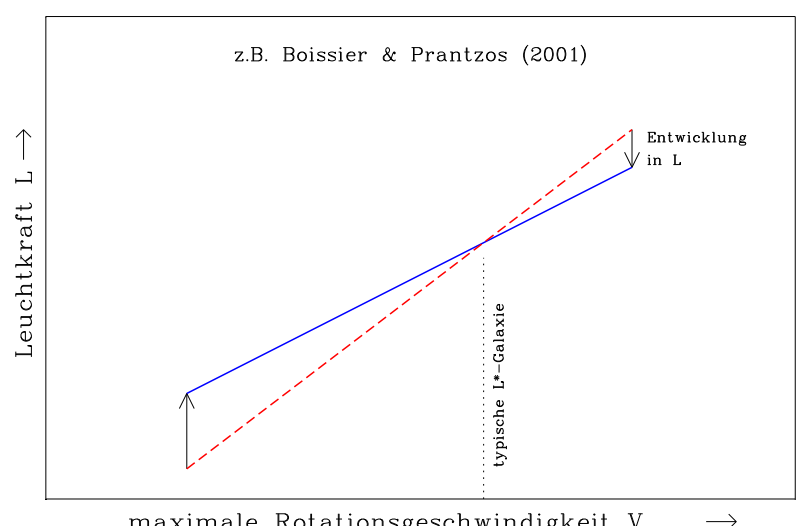

maximale Rotationsgeschwindigkeit $\mathrm{V}_{\max } \rightarrow$

Abbildung 3: Durch $N$-Körper-Simulationen vorhergesagte Entwicklung der Tully-FisherRelation (TFR) bei Berücksichtigung bestimmter massenabhängiger Prozesse. Die durchgezogene Linie repräsentiert die TFR von Spiralgalaxien im lokalen Universum, die gestrichelte Linie gibt die in den Simulationen gefundene TFR von weit entfernten Spiralen wieder. Massearme (langsam rotierende) Spiralgalaxien werden mit fortschreitendem Weltalter leuchtkräftiger, bei massereichen Galaxien wird eine Abnahme der Leuchtkraft prognostiziert. Galaxien etwa von der Masse der Milchstraße (sog. $L^{*}$-Galaxien) zeigen eine vernachlässigbare Entwicklung. Nähere Erläuterungen siehe Text.

Alter der Sterne.

Ein wesentliches Ziel der vorliegenden Arbeit war es also, diese eng mit dem hierarchischen Szenario verknüpften Vorhersagen zu testen. Die Vorteile gegenüber anderen Studien der Entwicklung entfernter Spiralgalaxien lassen sich dabei wie folgt zusammenfassen:

- Ein sehr umfangreicher Datensatz mit rund 100 Galaxien wurde beobachtet, während frühere Untersuchungen typischerweise nur 10-20 Objekte enthielten.

- Die hier vorgestellte Stichprobe deckt einen weiten Bereich von Massen und Spektraltypen ab, während in vielen früheren Arbeiten bestimmte Typen durch Auswahleffekte überrepräsentiert waren.

- Für den Hauptteil der Analyse wurden nur
Rotationskurven verwendet, welche die Region konstanter Rotationsgeschwindigkeit bei großen Radien überdecken. Dies ist wesentlich für eine verlässliche Messung von $V_{\max }$. In früheren Studien hingegen wurde die Qualität der Rotationskurven nicht berücksichtigt.

- Es wurde ein Konzept zur Korrektur der Beobachtungseffekte entwickelt, die bei der Messung von Rotationskurven weit entfernter Spiralgalaxien zum Tragen kommen. Insbesondere führt die geringe scheinbare Größe der Objekte zu einem starken „Ausschmieren" der Kurven durch die Luftunruhe (seeing) und den Einfluß der Spaltbreite.

Mit den gewonnenen Daten wurde unter anderem die Tully-Fisher-Relation bei einer mittleren Rotverschiebung von $\langle z\rangle \approx 0.5 \mathrm{kon}$ struiert, was einer Rückblickzeit von ca. 5 Gigajahren oder $35 \%$ des Weltalters entspricht, siehe Abb. 4. Die Leuchtkraft ist in dieser Darstellung durch die Absoluthelligkeit bei einer Wellenlänge von $\sim 430 \mathrm{~nm}$ ( $B$-band) im Ruhesystem wiedergegeben, wobei gilt:

$$
M_{B} \propto-\log L_{B}
$$

Im Gegensatz zu den Vorhersagen numerischer Simulationen, die auf dem hierarchischen Modell basieren, wird beobachtet, daß massearme (langsam rotierende), entfernte Spiralgalaxien leuchtkräftiger sind als ihre lokalen Gegenstücke, während massereiche Spiralen keine signifikante Leuchtkraftentwicklung zeigen. Die statistische Sicherheit dieses Befunds beträgt $>99 \%$.

Die Diskrepanzen zwischen den in früheren Studien abgeleiteten Entwicklungen der GalaxienLeuchtkraft lassen sich auf der Basis dieses Ergebnisses erklären als eine Kombination von Auswahleffekten und der Statistik kleiner Zahlen, da diese Arbeiten oft nur einen bestimmten Massenbereich abdeckten und aufgrund der kleinen Datensätze keine robuste Berechnung der 


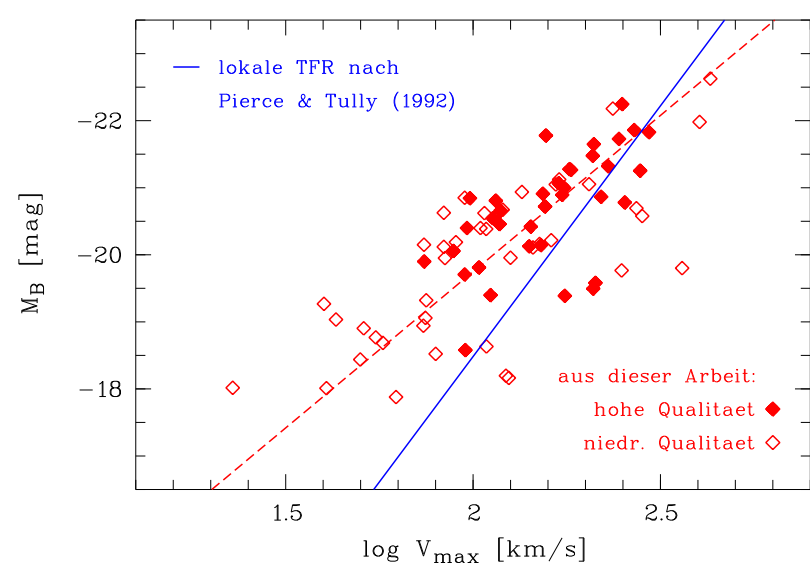

Abbildung 4: Tully-Fisher-Relation (TFR) von Spiralgalaxien aus der vorliegenden Arbeit, die eine mittlere Rotverschiebung von $\langle z\rangle \approx 0.5$ aufweisen. Volle Symbole entsprechen Objekten mit Rotationskurven hoher Qualität, die in den Bereich konstanter Rotationsgeschwindigkeit bei großen Radien reichen und daher robuste Werte für $V_{\max }$ liefern (volle Symbole); die gestrichelte Linie zeigt den Fit an diese Datenpunkte. Die durchgezogene Linie deutet die TFR von Spiralgalaxien im heutigen Kosmos an. Bei der TFR entfernter Galaxien wird eine geringere Steigung beobachtet, im Widerspruch zu den Vorhersagen numerischer Simulationen, die auf dem hierarchischen Szenario basieren.

Steigung der entfernten Tully-Fisher-Relation erlaubten. In manchen dieser Stichproben waren hauptsächlich massearme Galaxien enthalten, und mit der in der vorliegenden Arbeit gefundenen TFR läßt sich verstehen, warum in den entprechenden Studien auf eine starke Leuchtkraftentwicklung geschlossen wurde. Andere Untersuchungen berücksichtigten in erster Linie massereiche Spiralen und fanden daher nur moderate oder gar vernachlässigbare Leuchtkraftunterschiede zwischen lokalen und weit entfernten Galaxien.

Es wurden eine Reihe von Tests durchgeführt, um die Ergebnisse zu untermauern. So wird in Kapitel 6 unter anderem gezeigt, daß die geringe Steigung der entfernten Tully-FisherRelation nicht etwa auf einen Unvollständigkeitseffekt, Galaxienwechselwirkungen oder die be- grenzte räumliche Aufösung zurückgeführt werden kann.

Nach Ausschluß aller übrigen in Frage kommenden Effekte bleiben zwei mögliche Erklärungen für den Widerspruch zwischen den Beobachtungsergebnissen dieser Arbeit und den theoretischen Vorhersagen im Rahmen des hierarchischen Szenarios:

1. Die Sternbildung in massearmen Halos aus Dunkler Materie könnte über einen großen kosmischen Zeitraum hinweg durch den sog. UV-Hintergrund unterdrückt worden sein, der z.B. von Aktiven Galaktischen Kernen herrührt. Die Sterne in massearmen Spiralen wären dann erst bei Rotverschiebungen $z<1$ gebildet worden, was die starke Leuchtkraftentwicklung erklären könnte. Um eine Übereinstimmung mit den in dieser Arbeit abgeleiteten Virialmassen zu erreichen, müssten allerdings die in der Literatur angegebenen, theoretischen Massengrenzen für Halos mit unterdrückter Sternentstehung um rund zwei Größenordnungen (!) korrigiert werden.

2. Der Zusammenhang zwischen den Eigenschaften der Sternpopulation von Galaxien (d.h., ihrer baryonischen Materie) und ihrer Gesamtmasse (die von der Dunklen Materie dominiert wird) ist noch nicht hinreichend verstanden, um realistisch in den numerischen Simulationen berücksichtigt zu werden. Hinzu kommt das angesprochene Problem der geringen Massen-Auflösung. Möglicherweise werden zukünftige Fortschritte auf diesen Gebieten zu einer Modifikation des hierarchischen Modells selbst führen, denn — wie weiter oben angedeutet - es gibt auf kleinen räumlichen Skalen einige Beobachtungsbefunde, die mit der gegenwärtigen Theorie nur schwer in Einklang zu bringen sind. 
Deutsche Zusammenfassung 


\section{Chapter 1}

\section{Introduction}

During the last decade, astrophysics and cosmology have faced great scientific progress. Not only thanks to the improved computational power which led to detailed simulations of the universe on all spatial scales, but also due to the capability of the new generation of $10 \mathrm{~m}$-class telescopes like the Very Large Telescope (VLT, Chile) and Keck Observatory (Hawaii).

However, several topics remain puzzling. The paradigm of modern cosmology is the Cold Dark Matter hierarchical model. In this scenario, the vast majority of matter is non-luminous, nonbaryonic and interacts only gravitationally. It has been a very succesful tool for the understanding of structure formation in the early universe, in particular for quantitative modelling of the structure growth on large scales $(>1 \mathrm{Mpc})$.

Moreover, it offers a scheme for the evolution of galaxies (structures on the scales of $\mathrm{kpc}$ or tens of $\mathrm{kpc}$ ) by predicting that small-mass galaxies have formed first in the early cosmic stages, followed by an epoch of merger and accretion events during which larger galaxies were build up. Some aspects of this scenario have been confirmed by observations: e.g., spiral galaxies at high redshifts have smaller mean sizes than their present-day counterparts (e.g., Giallongo et al. 2001). But on the other hand, there also are topics where theory and observations are in contradiction. The situation is further complicated due to the fact that there are discrepant results among different observational studies. A detailed inventory on the current state of the de- bate will be given further below.

It was one of the basic motivations of this study to profundly test the hierarchical scenario with a large sample of galaxies that probes a significant fraction of the cosmic age and allows robust number statistics. Before the strategy of this thesis will be given in more detail, the basic concepts of the hierarchical model will be recalled (next section), as well as the main properties of galaxies in the local universe (section 1.2). Observational and theoretical studies revealed that some parameters of galaxies, like their luminosities and sizes, are tightly linked by so-called scaling relations. These will be described in section 1.3, and their application to studies of galaxy evolution is one of the major topics in section 1.4, where the current state of theory and observations will be addressed. Finally, the motivation of this study and a brief overview of the thesis will be the content of section 1.5.

\subsection{The Hierarchical Scenario}

The cosmological model which currently is widely favoured is that of a flat universe which is dominated by Cold Dark Matter (CDM) and Dark Energy. This cosmology is strongly supported by a variety of observational studies, which focussed on

- the Cosmic Microwave Background (more precisely, the location of the peaks in its angular power spectrum, see Spergel et al. 2003 for most recent results), 
- the Large Scale Structure of the universe (see Bahcall et al. 1999 for an overview),

- the Big Bang Nucleosynthesis (e.g., Burles \& Tytler 1998) and

- distant type Ia supernovae (e.g., Perlmutter et al. 1999)

Due to the common agreement on this model, it is often referred to as the "concordance" cosmology (e.g., Tegmark et al. 2001). In the formalism of Einstein's field equations, the Dark Energy can be associated with a non-zero cosmological constant $\Lambda$. For this reason, the concordance model often is abbreviated as " $\Lambda \mathrm{CDM}$ ". Normalised to the critical density $\varrho_{c}$, the observed values of the matter density $\Omega_{\mathrm{m}}$ and the density corresponding to the cosmological constant, $\Omega_{\Lambda}$, indicate a flat metric of the spacetime:

$$
\Omega_{\Lambda}+\Omega_{\mathrm{m}}=\Omega_{\mathrm{tot}}=1 .
$$

The contribution of baryonic matter to $\Omega_{\mathrm{m}}$ is only $\sim 10 \%$ in the concordance cosmology (e.g., Spergel et al. 2003).

Evidence for DM comes from a wide range of observations. E.g., the flat rotation curves of spiral galaxies have indicated a mass contribution of non-luminous matter (section 1.2.3). On Mpc-scales, clusters of galaxies yielded similar results. Under the assumption that the clusters are virialised systems, the velocities of the cluster member galaxies and the temperature of the hot, $\mathrm{X}$-ray emitting intra cluster gas are by far too large to be explained by the gravitational potential of the baryonic matter (e.g., Castillo-Morales \& Schindler 2003). Gravitational lensing events, where the light of high-redshifted objects is amplified (so-called strong lensing) or their shapes are distorted (weak lensing) by foreground clusters in the line-of-sight, also confirmed that the majority of the cluster masses consist of DM (ibid.).

One important consequence of a CDMdominated cosmology is that the Jeans mass, which is the upper limit on the mass of an overdense region that does not become gravitationally unstable, has a small value $\left(\sim 10^{6} M_{\odot}\right.$, which is of the order of globular stellar clusters, e.g., Longair 1998) in the early universe. After recombination, the baryonic matter fell into the DM potential wells, cooled via radiation and began to form stars. Large structures successively were build up from smaller structures. Such a framework of structure formation is called hierarchical merging or "bottom-up" universe.

This scenario has been very successful for the understanding of the observed large-scale structure of the universe (see, e.g., Peacock 2003 for a recent overview). These structures, which range from clusters and superclusters of galaxies to scales of $\sim 100 \mathrm{Mpc}$ (like the Great Wall, Geller \& Huchra 1989), and are separated by so-called voids which have a very low mass density, are well reproduced in $N$-body simulations that are based on CDM (e.g., Doroshkevich et al. 1999).

For the particular subject of individual galaxies, the hierarchical scenario predicts that low-mass galaxies have formed at first in the early cosmos and that larger galaxies consecutively were build up via merging or accretion of smaller systems. At least for elliptical galaxies (see next section), one fundamentally different scenario exists according to which these objects formed at high redshift and have undergone a passive evolution since then, meaning that they only evolved in terms of the stellar population. This "monolithic collapse" model (Eggen, Lynden-Bell \& Sandage 1962, Sandage 1986) will not be in the focus of this thesis which uses the data of spiral galaxies.

While some predictions of the hierarchical model for the evolution of individual galaxies are confirmed by observations, there also are various indications for shortcomings. Before the pros and cons are given in detail, the basic properties of galaxies in the local universe will be recalled in the next two sections. 


\subsection{Types of Galaxies}

Galaxies in the local universe can be devided basically into three categories: Ellipticals, spirals and irregular galaxies. The two former types show fundamental differences in their morphological shapes (the distributions of the luminous matter), spectra and kinematics. Irregular galaxies merely are characterised by the fact that they do not match the properties of ellipticals nor spirals. They often are gas-rich and of low mass.

In the follwing, three aspects which can be used to establish a classification scheme will be separately addressed (for a more detailed introduction see, e.g., Combes et al. 1995). Emphasis will be placed on the kinematics of spiral galaxies (section 1.2.3), because these were used as a basic tool for the determination of distant spiral evolution in this study.

\subsubsection{Morphology}

The classification of galaxies in terms of their morphology, or more precisely, the distribution of their luminous matter, has been initially introduced by E. Hubble (1926). It is also known as the "Hubble tuning fork", see Fig. 1.1.

Elliptical galaxies, which are sub-classified from E0 to E7 according to their axial ratio, basically feature a one-component shape and a luminosity profile that is proportional to $r^{1 / 4}$ (where $r$ is the galactocentric radius), known as the "de Vaucouleurs"-profile.

Spiral galaxies, on the other hand, basically contribute two components: A central bulge, characterised by a de Vaucouleurs profile, and a surrounding disk with an exponential profile proportional to $\mathrm{e}^{-r / r_{\mathrm{d}}}$. Here, $r_{\mathrm{d}}$ is the disk scale length, which gives the characteristic size of the disk component. Spirals are sub-classified from Sa to Sd (the latter sub-type is not shown in Fig. 1.1) according to the fractional size of the inner bulge and the size of the spiral arms. The presence of spiral structure is well understood

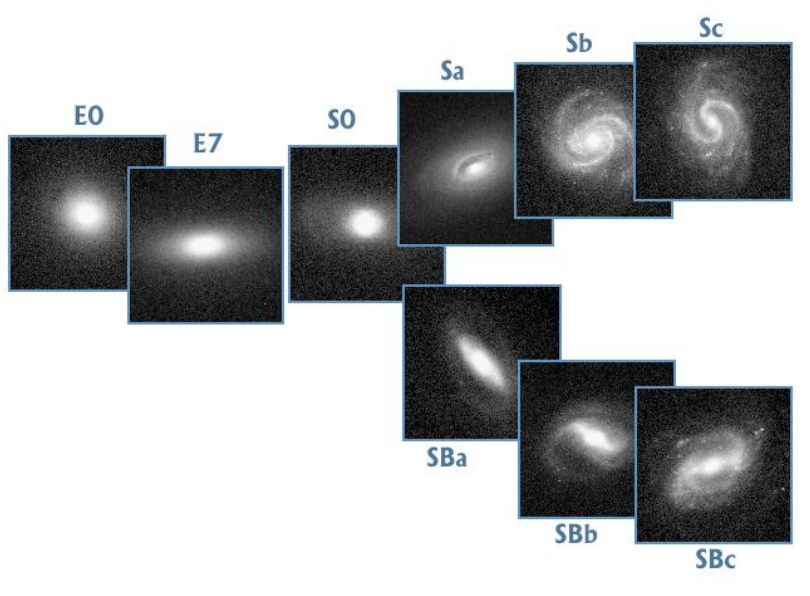

Figure 1.1: The Hubble "tuning fork" for galaxy morphology classification, ranging from early-type galaxies (ellipticals and S0 galaxies) on the left to late-type galaxies (spirals) on the right hand side. Spirals are sub-classified according to the fractional size of the central bulge component, which decreases from Sa to Sc, and the size of the spiral arms which increases from left to right. The presence of a central bar is denoted by a "B" in the lower panel. Very latetypes (Sd spirals and irregular/peculiar galaxies) are not displayed (C) Z. Frei and J. E. Gunn, Princeton University Press 1999).

in the framework of density wave theory (e.g., Binney \& Tremaine 1987). In the case of the presence of a central bar, the nomenclature is changed to $\mathrm{SBa} \ldots \mathrm{SBd}$.

A class of objects which do not fit in either of the above schemes are the irregular galaxies (not shown in Fig. 1.1, abbreviated Im). These include systems which are isolated, but have a peculiar isophotal shape, neither following a de Vaucouleurs nor an exponential profile, and also systems which clearly are in interaction with neighboring objects and are distorted via tidal forces or even undergo a merging event.

The so-called lenticular or S0 galaxies are located between ellipticals and spirals in the Hubble scheme. Ellipticals and S0 galaxies are also referrred to as early-type galaxies, spirals and irregulars as late-types. Morphologically, S0 galaxies feature disks like spirals, whereas kine- 
matically, they are early-type galaxies (see section 1.2.3).

It has been found that the fractional contributions of the various Hubble types to the galaxy population depend on the environment or, more precisely, the galaxy number density. In the field, spirals are the most frequent morphological type, whereas in clusters of galaxies, and in particular the cluster centers, the E/S0 types make up the major part of the population. This is known as the morphology-density relation (Dressler 1980). A more quantitative way of morphological classification is the bulge-to-total ratio $B / T$ which gives the contribution of the bulge component to the total luminosity. This parameter decreases from early to late-type galaxies (e.g., Efstathiou \& Silk 1983), with the highest values for ellipticals and the lowest values for late-type spirals and irregulars (reaching $B / T=0$ in the case of no detectable bulge).

A similar concept are the concentration index $C$ and the asymmetry index $A$ introduced by Abraham et al. (1996). The former is sensitive to the steepness of an objects luminosity profile and decreases from early to late-types, while the latter gives the deviation of a profile from symmetry and increases from early to late-types. An advantage of this classification scheme is its reliability for a morphological classification even at relatively low spatial resolution. It is therefore especially useful for distant galaxies of small apparent sizes and has been used in this thesis also, see chapter 4.5 for details.

\subsubsection{Spectral Energy Distribution}

The morphological Hubble sequence correlates tightly with the properties of the Spectral Energy Distribution (SED). On the average, the broad band colors become bluer from early to late-types. The central bulges of spirals, however, merely show colors which are typical for E/S0 galaxies, whereas their disks are bluer.

A similar trend is observed in the integrated spectra (in the following, only the rest-frame

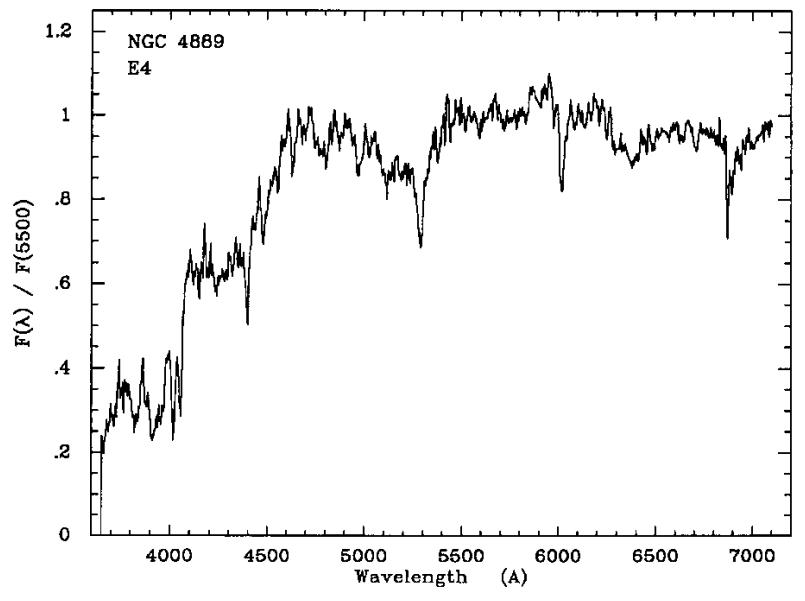

Figure 1.2: $\quad$ Spectrum of an elliptical galaxy from Kennicutt (1992a).

optical regime will be considered). While E/S0 galaxies feature strong absorption lines (in particular, the Balmer series, Ca II and $\mathrm{Mg}_{b}$ ) and only weak emission (if any), the strength of emission lines (most prominent typically are $\mathrm{H} \alpha$, [O II] 3727, H $\beta$ and [O III] 5007) on the mean increases from Sa to Im, whereas the absorption line strength decreases. While the strength of absorption is indicative for the metallicity (the fraction of elements heavier than $\mathrm{He}$ ) and the age of the stellar population, the emission line strength is correlated with the fraction of highmass stars in the stellar content. For earlytype spirals, $\mathrm{H} \alpha$ is the strongest emission line, whereas for very late-types, this mostly is the [O III] 5007 line, indicating a significant fraction of very young, hot stars with $>30 M_{\odot}$ (Bik et al. 2003). Examples for an elliptical, an Sc and an $\mathrm{Sm} / \mathrm{Im}$ spiral from the Kennicutt catalogue (1992a) are shown in Figs. 1.2 to 1.4. Note that these spectra were normalised to the flux at $5500 \AA$.

When the Star Formation History of the temperal evolution of the Star Formation Rate (SFR) $\psi(t)$ of a galaxy is approximated with an exponential law of the form $\psi(t) \propto \mathrm{e}^{-t / \tau}$, where $\tau$ is the characteristic e-folding time, then $\tau$ increases from early to late-types (Struck-Marcell 


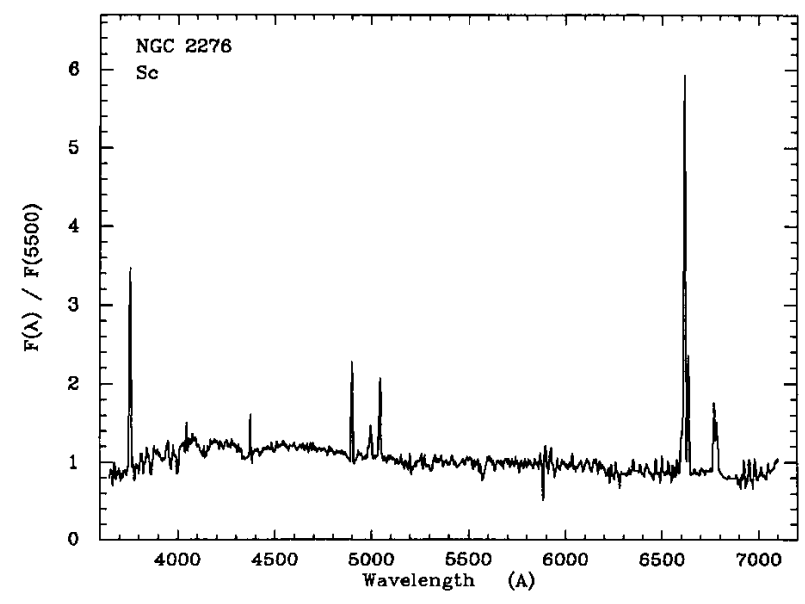

Figure 1.3: Spectrum of an Sc spiral from Kennicutt (1992a).

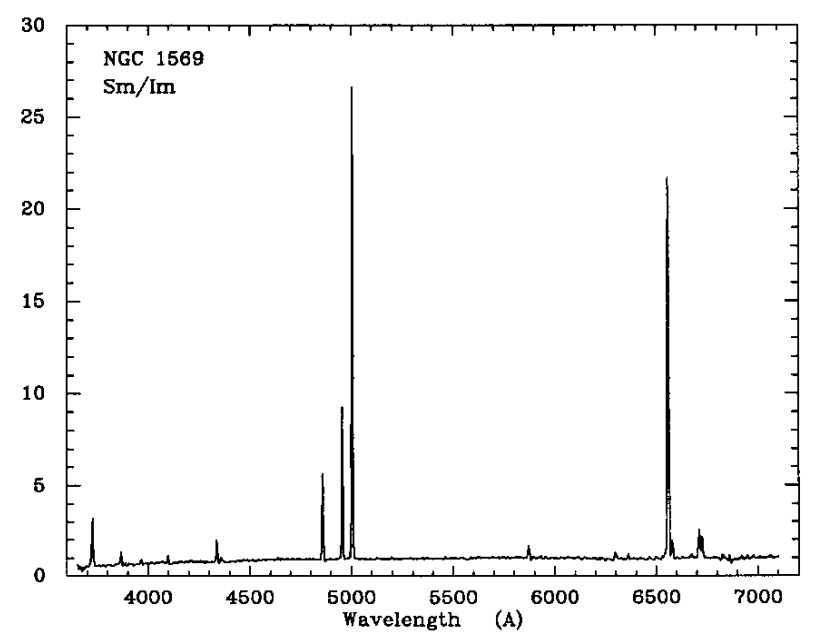

Figure 1.4: Spectrum of a very late-type galaxy from Kennicutt (1992a).

\& Tinsley 1978). This explains the color trend in the local Hubble sequence: The stellar population of early-type galaxies indicates a short starburst at high redshift, followed by a quiescent evolution during which the population becomes old and the short-lived blue, high-mass stars are destroyed in supernovae. On the other hand, the SFR of late-type galaxies shows a less steep decrease or may be even constant, which results in a non-negligible fraction of high-mass stars and thereby blue colors.

\subsubsection{Kinematics}

The kinematics of galaxies, i.e., the movement of their stars and gas, also show different classes. Since galaxies are virialised systems, their potential energy (self-gravitation) is counter-balanced by the kinetic energy of all mass particles. A galaxy may either be stabilised due to random motion or ordered rotation. The key parameter of the former is the velocity dispersion $\sigma_{\mathrm{v}}$, while the latter is characterised by the rotation velocity, $V_{\text {rot }}$. Both quantities usually are a function of galactocentric radius.

Early-type galaxies (ellipticals and S0 galaxies) and the bulges of spirals are observed to be stabilised by their velocity dispersion, which exceeds their rotation velocity at all radii. In other words, these systems are dynamically hot (Dynamically Hot Galaxies, DHGs, e.g., Dressler et al. 1987). Spiral galaxy disks, on the other hand, are stabilised due to rotation, hence dynamically cold. The source of the angular momentum may be tidal torques between neighboring density fluctuations during the process of disk formation (e.g., Silk 2000). Irregular galaxies usually show rotation, but their kinematics are not as symmetric as those of normal spirals, and peculiarities are more frequent (Sofue \& Rubin 2001).

The dependence of $V_{\text {rot }}$ on the radius has been firstly derived by Freeman (1970). For the case of a constant mass-to-light ratio and an infinitely thin disk, the exponential light profile directly transforms into an exponential mass surface density $\mu$ :

$$
\mu(r)=\mu_{c} \mathrm{e}^{-r / r_{\mathrm{d}}}
$$

Freeman showed that in the state of centrifugal equilibrium, $V_{\text {rot }}(r)$ linearly rises for increasing $r$, peaks at $r=2.2 r_{\mathrm{d}}$ and then converges to a Keplerian decline:

$$
V_{\text {rot }}\left(r>>2.2 r_{\mathrm{d}}\right) \propto r^{-1 / 2} .
$$

However, the situation is different for observed rotation velocities as a function of radius, called 


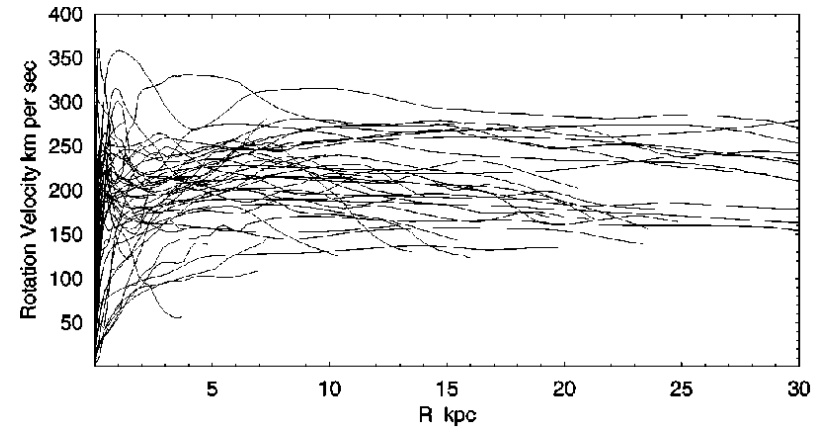

Figure 1.5: An overlay of many rotation curves of local spirals from Sofue \& Rubin (2001). The majority of the curves do not show a Keplerian decline, as would be expected for an exponential disk mass density, but a nearly constant rotation velocity out to the farthest measured points.

rotation curves (RCs). These curves are derived by a measurement of the Doppler shifts of spectral features due to the line-of-sight rotation velocity, either in the radio regime (using, e.g., the $21 \mathrm{~cm}$ line or $\mathrm{CO}$ ), the optical (based on the Balmer lines, [O II] or [O III] emission) or the near-infrared (e.g., to use the $\mathrm{H} \alpha$ emission of distant spirals).

Most observed RCs do not show a Keplerian decline, but a constant value $V_{\text {rot }}(r)=V_{\max }$ at large radii (see Fig. 1.5). This indicates a nonluminous mass component that dominates the mass distribution at large radii. In particular, a linear dependence of the total mass on the radius is implied: $M_{\text {tot }}(r) \propto r$.

The flat RCs of spirals have been one of the basic motivations for the introduction of Dark Matter. An alternative approach are the Modified Newtonian Dynamics (MOND, Milgrom 1983). In this hypothesis, the Newtonian law of gravity has to be modified in the regime of very small accelerations. Though MOND can be used to explain the flat RCs without DM, this is still an ad hoc theory. Moreover, it is not reliable to explain a range of large-scale phenomena like, e.g., the observed amount of gravitational lensing.

It should be emphasised that the correlation between morphological type, SED type and kine- matics, which has been described in the last three sections, is basically a characteristic of galaxies in the local universe. The debate on whether or not this correlation is also present at earlier cosmic epochs is still ongoing. E.g., observations at redshifts $z>1$ indicate that the fraction of peculiar morphologies is larger than in the local universe. This topic will be addressed in section 1.4.

In the following, a fundamental tool for the observation of galaxy evolution will be introduced.

\subsection{Scaling Relations}

In 1977, Tully and Fisher observed a correlation between the luminosity $L$ and the maximum rotation velocity $V_{\max }$ in a sample of nearby spiral galaxies. Since the luminosity is equivalent to the absolute magnitude $M \propto-\log L$, this Tully-Fisher Relation (TFR, Tully \& Fisher 1977) can be parameterised as

$$
M_{X}=a \log V_{\max }+b,
$$

where $X$ is the considered filter passband, $a$ is the slope and $b$ the zero point. The slope is negative for a TFR in this form; faster rotators feature higher luminosities than slow rotators. This can be understood as a combination of the virial theorem (remember that the disks are centrifugally supported) and a nearly constant mass-tolight ratio (e.g., Koda, Sofue \& Wada 2000b). The TFR slope $a$ is steeper for redder wavelengths of the passband $X$, mainly due to the bluer colors of slow rotators (e.g., Rubin et al. 1985). This will be a key factor for the interpretation of previous TFR studies on the basis of this thesis in chapter 6 . A striking feature of the TFR is its small scatter, which may have an intrinsic value of zero in the $K$-band (Verheijen 2001). However, the observed value of the scatter includes errors on, e.g., the disk inclination angle.

The TFR of a large sample of local spirals with systematic velocities $V_{\text {sys }}<15000 \mathrm{~km} / \mathrm{s}$ from 


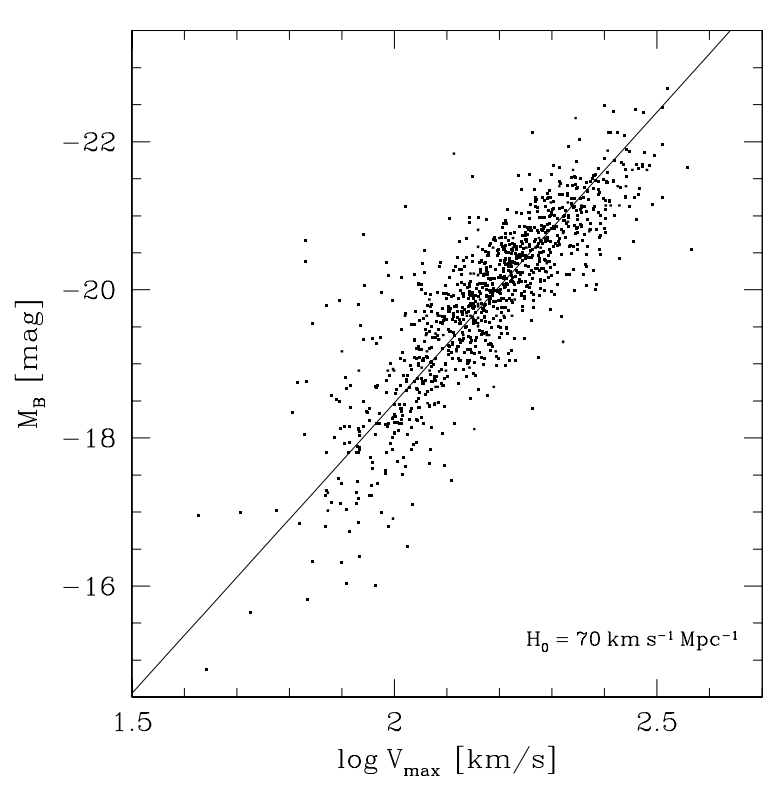

Figure 1.6: Tully-Fisher diagram of a large sample of local spirals from Haynes et al. (1999). The solid line denotes the bisector fit with a slope of $a=-7.85$.

Haynes et al. (1999) is shown in Fig. 1.6. A bisector fit (see section 6) to the 1097 objects yields a slope $a=-7.85$ and a zero point $b=-2.77$. Note that very faint objects with absolute magnitudes $M_{B}>-18$ are apparently underluminous for their maximum rotation velocity. This can be attributed to the high gass mass fraction in these galaxies (e.g., McGaugh et al. 2001), which contributes to the total mass and $V_{\max }$ (since $M_{\text {tot }} \propto V_{\text {max }}^{2}$, e.g., van den Bosch 2003), but not to the luminosity.

Within the last few years, the Tully-Fisher relation has been put into the framework of a Fundamental Plane (FP) for spiral galaxies that introduces the disk scale length $r_{\mathrm{d}}$ as a third parameter (e.g., Burstein et al. 1997). This is similiar to the FP of dynamically hot, early-type galaxies (e.g., Dressler et al. 1987), which correlates the velocity dispersion $\sigma_{\mathrm{v}}$, the effective radius $r_{\mathrm{e}}$ and the surface brightness within the effective radius, $\mu_{\mathrm{e}}$.

An illustration of the spiral FP is shown in Fig. 1.7, note that slightly different naming con-

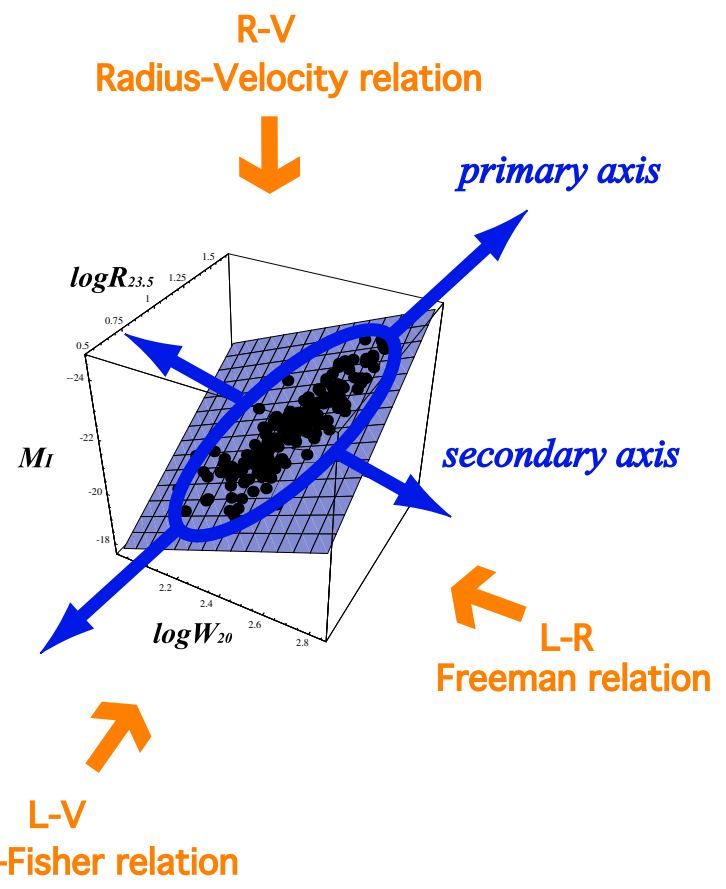

Figure 1.7: Three basic parameters of spiral galaxies are correlated via a Fundamental Plane (taken from Koda, Sofue \& Wada, 2000a). These are the luminosity (given here as the absolute $I$-band magnitude $M_{I}$ ), the maximum rotation velocity of the disk (given by the doppler-broadened $\mathrm{H}_{\mathrm{I}}$ linewidth) and the disk size (given here as the isophotal radius at an $I$-band surface brightness of $23.5 \mathrm{mag}$ ).

ventions and parameterisations are used in this graph. The three basic scaling relations of spirals, namely the TFR, the Freeman relation (correlating luminosity $L$ and disk size) and the velocity-size relation (between $V_{\max }$ and disk size) are projections of this FP. Within this plane, the distribution of spirals has an elongated shape, which may be understood in terms of two dominant parameters for disk galaxies, for example the mass and angular momentum (Koda, Sofue \& Wada 2000a).

For the matter of galaxy evolution, the scaling relations are powerful tools. Via a comparison between a distant sample of galaxies (which corresponds to an earlier cosmic epoch) and a local sample (representative for the present-day gal- 
axy population), scaling relations can be used to quantify the evolution of several parameters. With the velocity-size relation, e.g., it is possible to derive the evolution of disk sizes, which should be smaller for distant galaxies according to the hierarchical scenario.

The TFR, on the other hand, can be used to derive the evolution in luminosity. Due to the on average younger stellar populations - hence a higher fraction of massive, hot and luminous stars - at high redshifts, distant spirals should be more luminous for a given mass than their present-day counterparts. In particular, it is still an open question whether there is an evolution of the slope of the TFR with look-back time, which would be indicative for a mass-dependence of the luminosity evolution. Note that the scaling relations introduce an important advantage over studies of the luminosity function (the number density as a function of luminosity). An evolution of the latter with redshift only indicates an evolution of the luminosity density, but not necessarily of the luminosity of individual galaxies. In particular, in cannot be concluded whether distant galaxies are overluminous for their mass. The main part of the analysis in this thesis will be based on the TFR, nevertheless the velocitysize relation is used also. In the following, the current state of theory and observations in the field of galaxy evolution will be described, the latter with an emphasis on the results of TFR studies.

\subsection{Galaxy Evolution}

\subsubsection{Theory}

Theoretical studies mostly are based on numerical $N$-body calculations to simulate the structure growth from primordial density perturbations to the present-day universe. It has previously been noted that these simulations have been succesfully used to reproduce the Large Scale Structure. Moreover, the slope and scatter of the simulated local TFR were in good agreement with the observed values (e.g., Mo, Mao \& White 1998, Steinmetz \& Navarro 1999). In these studies, the TFR slope was predicted to remain constant with cosmic look-back time. However, the numerical zero points were offset by more than one magnitude from the observed ones. This was due to DM halos which had steeper density profiles at small radii then is implied by observations with high intrinsic spatial resolution. In effect, the simulated disks were too small and had too low values for the angular momentum (e.g., Navarro \& Steinmetz 2000). This is known as the "cuspy cores problem", one of the fundamental problems of the current CDM cosmology on small scales. A further example of its shortcomings is the "missing satellites problem". In the simulations, a large number of sub-clumps are produced in massive DM halos, which should result in a large number of small-mass satellite galaxies in the present-day universe. However, the observed number densities are orders of magnitude smaller than predicted. See, e.g., Sellwood \& Kosowsky (2001) for a review of these and other puzzling discrepancies between theory and observations.

A realistic modelling of the stellar content remains a challenge in $N$-body simulations, since individual stars are not resolved. Some studies have used parameterisations between stellar population properties and $V_{\max }$ to account for mass-dependent aspects of the chemospectrophotometric evolution.

For example, Boissier \& Prantzos (2001) used the "hybrid" approach (Jimenez et al. 1998) that relates the disk surface density to the properties of the associated DM Halo, and calibrated it to reproduce the observed colors of local spirals. Compared to these, the authors predict higher luminosities for large disks and lower luminosites for small disks at redshifts $z>0.4$. A similiar evolution is found by Ferreras \& Silk (2001). By modelling the mass-dependent chemical enrichment history of disk galaxies with the local TFR as a constraint, the authors find a TFR slope 
that increases with look-back time.

An important point about these models is that they generally need to be calibrated to the properties of local galaxies in order to be consistent with observations. Without this, the observed mass-dependence of the colors of spirals is not reproduced, in the sense that simulated massive spirals are too blue (e.g., Bell et al. 2003).

One important addition to the hierarchical scenario may arise from the so-called reionisation era. In the redshift regime $1<z<6$, a background of ultra-violet radiation from Active Galactic Nuclei (massive black holes surrounded by an accretion disk, see Osterbrock 1993 for a review) and starbursting galaxies could have suppressed the star formation in DM halos with masses below $10^{9} M_{\odot}$ (e.g., Babul \& Rees 1992). In effect, dwarf galaxies would have begun to form stars at relatively low redshifts $z<1$ and hence would have young stellar populations. This offers an explanation for the excess of faint, blue galaxies that is observed in galaxy number counts (see Ellis 1997 for an overview).

\subsubsection{Observations}

Straightforwardly, one would expect that in a hierarchical universe, the sizes of spiral disks decrease with increasing look-back time. This is in fact indicated by various observations (e.g., Giallongo et al. 2000, Mao, Mo \& White 1998). Moreover, the colors and morphologies of galaxies in the Hubble Deep Field North (Williams et al. 2000) indicate that the formation of the local Hubble sequence has taken place in the regime $1<z<2$ (Kajisawa \& Yamada 2001). Galaxies at redshifts $z>2$ are either peculiar — with one third of the total population undergoing merging events - or centrally concentrated "blobs". At redshifts $0.5<z<1$, the frequency of peculiar spirals is significantly higher than in the local universe (a recent review is given in van den Bergh 2002). All these findings fit well into the framework of hierarchical merging.

Based on the luminosity function in the regime $0<z<0.5$, Driver et al. (1996) estimated a mean decrase of $\sim 1.5 \mathrm{mag}$ in the rest-frame $B$ luminosity for very late-types. However, as previously stated, the TFR is a more powerful tool for the quantification of this evolution.

In the last decade, many studies of the local TFR have produced very large samples with $N_{\text {obj }} \approx 1000$ (e.g., Haynes et al. 1999), not only to derive the slope and scatter with high accuracy, but also to map the peculiar velocity field out to $c z \approx 15000 \mathrm{~km} \mathrm{~s}^{-1}$ (e.g., Mathewson \& Ford 1996). Other groups used spirals, partly with cepheid-calibrated distances, to measure the Hubble constant. For example, Sakai et al. (2000) derived a value of $H_{0}=(71 \pm 4) \mathrm{km} \mathrm{s}^{-1}$ with this method.

It is still matter of debate whether the TFR depends on galaxy number density, i.e., whether the field and cluster relations are different. The kinematics of cluster spirals could be disturbed due to gravitational interaction in close encounters of cluster members or via ram-pressure from the intra-cluster medium. In fact, RCs of cluster spirals are observed to be frequently asymmetric or truncated (e.g., Rubin, Waterman \& Kenney 1999). Although the main body of the data set for this thesis consists of field spirals, the question of a TFR dependence on environment will be subject to chapter 6.4.3.

At higher redshifts, robust measurements of rotation velocities become increasingly difficult. Partly because of the small apparent brightness of the galaxies, but also due to their small apparent size which limits the intrinsic spatial resolution. The latter introduces a beam smearing effect that has to be accounted for in the data analysis (see chapter 5.2 for details). Note that all of the studies which are cited in the following used spectroscopy in the optical and the near infrared. Radio observations of spiral kinematics are restricted to the low-redshift regime.

A couple of samples with $10-20$ objects in the range $0.25<\langle z\rangle<0.5$ were observed in the last years to estimate a possible evolution in luminos- 
ity by comparison to the local TFR. The results of these studies were, however, quite discrepant: Vogt et al. (1996, 1997), e.g., find only a modest increase in the rest-frame $B$ luminosity of $\Delta M_{B} \approx-0.5^{m}$ (remember that smaller absolute magnitudes correspond to larger luminosities). In contrast to this, Simard \& Pritchet (1998) and Rix et al. (1997) derive a much stronger brightening with $\Delta M_{B} \approx-2.0^{m}$. A study of 19 spirals by Milvang-Jensen et al. (2003) exhibits a value of $\Delta M_{B} \approx-0.5^{m}$ and shows evidence for an increase with redshift. Another sample of 19 spirals by Barden et al. (2003) which covers the high redshifts $0.6<z<1.5$ yields a value of $\Delta M_{B} \approx-1.1^{m}$.

It seems likely that some of these results are affected by the selection criteria. For example, Rix et al. selected galaxies on blue colors with $(B-R)_{\mathrm{obs}}<1.2^{m}$ and Simard \& Pritchet on strong [O II] emission with equivalent widths $>20 \AA$, while Vogt et al. partly chose large disks with $r_{\mathrm{d}}>3 \mathrm{kpc}$. On the one hand, strong emission lines (corresponding to a high signal-tonoise ratio) or large disks (introducing an improved sampling of the intrinsic rotation velocity field) simplify the analysis. But on the other hand, the samples are biased towards certain spiral sub-types. While a selection on blue colors or strong emission lines prefers late-type spirals, the large disk criterion leads to the overrepresentation of massive, early-type spirals. Additionally, due to the small samples, all these studies had to assume that the local TFR slope holds valid at intermediate redshift. A further discussion of this particular issue as well as the discrepancies in $\Delta M_{B}$ will follow in chapter 6.5.

Based on a larger data set from the DEEP Groth Strip Survey (Koo 2001) with $N_{\text {obj }} \approx 100$ spirals in the range $0.2<z<1.3$, Vogt $(2000,2001)$ finds a constant TFR slope and a negligible restframe $B$-band brightening of less than $0.2 \mathrm{mag}$. However, in a more recent publication from this group which investigates the luminositymetallicity relation, an evolution both in slope and zero point is observed (Kobulnicky et al. 2003). The luminosity offsets are largest at the low-luminosity end of the sample and smallest at the high-luminosity end. The authors argue that low-luminosity galaxies probably have undergone a decrease in luminosity, combined with an increase of the metallicity, in the last $\sim 8$ Gyrs. This is at variance with the results from Vogt $(2000,2001)$.

\subsection{Motivation and Overview}

Though the scaling relations are potentially powerful tools for the analysis of spiral galaxy evolution, most of the previous studies in this field have suffered either from small number statistics or from selection effects which introduced biases in the results. To avoid these shortcomings, the study described in this thesis was based on the FORS Deep Field (FDF, see chapter 2.1 and Appenzeller et al. 2000), a sky region which has been imaged with very long exposure times using the VLT. Thanks to the very deep multiband photometry of the FDF, it was feasible...

- to select galaxies for spectroscopy from a large field-of-view $\left(\sim 40 \mathrm{arcmin}^{2}\right)$ and thus from a large number of candidates.

- to use photometric redshifts in the selection process, i.e., the SED types and approximated distances of all candidates were known. Therefore, any biases arising from a specific selection (e.g., on blue colors) could be avoided. All spectral types, ranging from Sa to Sdm/Im, could be included.

- to construct a large sample $\left(N_{\mathrm{obj}} \approx 100\right)$ of galaxies covering redshifts up to $z=1$, corresponding to a look-back time of $>50 \%$ of the age of the universe $(\sim 14$ Gyrs according to recent studies, e.g., Spergel et al. 2003).

Using the VLT in Multi Object Spectroscopy mode, spectra of 129 spirals were taken in total, mainly in Oct./Sep. 2000 and Oct. 2001. The basic aims of this project were the following: 
- Derivation of spatially resolved rotation curves of the spirals.

- Construction of a robust TFR at intermediate redshift.

- Quantification of the luminosity evolution of the galaxies over a significant fraction of the age of the universe.

- Test of a potential evolution of the TFR slope with look-back time (which would point to a mass-dependency of the luminosity evolution); interpretation of the results in the framework of hierarchical merging.

- Test of a possible evolution of the disk sizes that is predicted by simulations based on the hierarchical scenario.

- To verify, whether the correlation between morphological, spectral and kinematical type - which characterises the Hubble sequence of local galaxies - is also valid for spirals at intermediate redshifts.

- Derivation of the total (virial) masses of the objects.

These issues are going to be adressed in chapters 4 to 6 . Before this, in chapter 2, it will be described how the FDF object catalogue was used to construct a sample for the VLT spectroscopy. Details of the telescope configuration and the observation strategy are given.

The spectroscopic data reduction steps are specified in chapter 3. Furthermore, the design of a special high-resolution image of the FDF for the purpose of light profile analysis is depicted.

All aspects of the spectrophotometric analysis are described in chapter 4 . This includes the redshift determination, SED classification, description of the program used for luminosity profile fitting and tests of the program, measurements of the structural parameters, morphological classification and the computation of the luminosities.
Chapter 5 comprises the kinematic analysis. Here, the extraction of the rotation curves and the derivation of $V_{\max }$ are described. The latter is achieved via a simulation of the intrinsic velocity fields, which is mandatory to account for the observational effects in the case of distant, apparently small spirals. It will be investigated whether different assumptions on the intrinsic rotation curve shape affect the results.

The evolution of the scaling relations is the main topic of chapter 6 . After a discussion of the appropriate local reference sample, the distant TFR and the velocity-size relation will be constructed. It will be shown that the distant TFR has a decreased slope with respect to samples of spirals in the local universe. Various tests are applied to confirm that this result is not due to systematic errors or peculiarities of the spirals in the data set. The tests include a potential incompleteness bias, galaxy-galaxy interactions, a comparison of various templates of intrinsic rotation curves, different conventions for the correction of intrinsic dust reddening, the impact of the spatial resolution etc. Discrepancies between previous observational studies will be explained on the basis of the findings, and the impact on the hierarchical scenario will be discussed.

The thesis is summarised in chapter 7 , which also comprises a brief outlook on the continuation of this project.

Unless otherwise stated, the concordance cosmology will be assumed throughout this thesis, i.e., a flat universe with $\Omega_{\mathrm{m}}=0.3, \Omega_{\Lambda}=0.7$ and $H_{0}=70 \mathrm{~km} \mathrm{~s}^{-1} \mathrm{Mpc}^{-1}$. These values are in compliance with the most recent analysis of the power spectrum of the Cosmic Microwave Background observed by WMAP (Wilkinson Microwave Anisotropy Probe, Spergel et al. 2003). 


\section{Chapter 2}

\section{Construction of the Sample}

It can already be concluded from the outline of the previous studies in the last chapter that any attempt to robustly derive the evolution of the scaling relations of spiral galaxies has to fulfill a couple of requirements. In particular, it is important to construct a sufficiently large sample $\left(N_{\text {obj }} \gg 20\right)$ and choose the selection criteria very carefully in order to avoid a bias towards certain galaxy sub-types.

The latter criterion is in turn tightly linked to the quality of the data on which the selection process is based. If the imaging data are restricted to two filters, only one color index will be available to discriminate between early and late-type galaxies. Rix et al. (1997), e.g., have chosen a constraint of $B-R<1.2$. If additional low-resolution spectroscopy is available, it will be feasible to select objects which show emission lines, as was the case in the study of Simard \& Pritchet (1998), who chose galaxies with [O II] equivalent widths above $20 \AA$ for their followup spectroscopy. Both these selection criteria result in data sets which mainly comprise latetype spirals. As will be shown in chapter 6.5, this introduces a bias towards low-mass galaxies with a large luminosity evolution over the past few Gyrs.

For these reasons, the TF sample was selected from a sky region with very deep, multi-band photometry, the FORS Deep Field (FDF). This way, it was possible to discriminate between ellipticals and spiral galaxies without neglecting early-type sprials. Furthermore, photometric redshifts were taken into account in the selection process to ensure the visibility of emission lines in the usable wavelength range of the spectrograph. And thirdly, the apparent magnitudes could be derived very accurately (with errors $\left.\sigma_{m} \ll 0.1^{m}\right)$ even for the faintest objects in the spectroscopic sample. Note that the Hubble Deep Fields (HDFs, Williams et al. 1996, 2000) were not appropriate for this project because of the small field-of-view (FOV): Based on a catalogue by Marleau \& Simard (1998) with structural parameters of more than 500 galaxies with $I<26$ from the HDF-North, less than 20 (!) spirals would meet the main selection criteria (see section 2.3).

\subsection{The FORS Deep Field}

It was the primary aim of the FDF project to perform multi-band imaging in the optical and near-infrared with visible limiting magnitudes comparable to the HDFs but with a substantially larger FOV. The main scientific driver was the construction of a cosmic census on galaxy evolution via a combination of the photometry with extensive follow-up spectroscopy. The instrument for the optical regime was the Focal Reducer / Low Dispersion Spectrograph (FORS, see Appenzeller et al. 1998 or www.eso.org/instruments/fors1/ for an overview) which has been designed and built in a collaboration of the Universitäts-Sternwarte Göttingen, the Landessternwarte Heidelberg and the Univer- 
sitätssternwarte München. Since 1999, two versions of the FORS instrument (named FORS1 \& FORS2) are mounted on two units of the Very Large Telescope (VLT) which is operated by the European Southern Observatory (ESO) at Cerro Paranal, Chile.

In return for their contributions, the three named institutes were given a pool of guaranteed observing time (so-called Guaranteed Time Observations, GTOs) by ESO. A substantial fraction of this GTO was reserved for the FDF project, which has been firstly outlined in Appenzeller et al. (2000).

In the search for a suitable sky area for the deep photometry, several criteria were combined. A rigid limit on the galactic extinction was set $\left(E(B-V)<0.02^{m}\right)$, and the field should be devoid of large, nearby galaxies, bright stars $\left(V<18^{m}\right)$ within the FOV and very bright stars $\left(V<5^{m}\right)$ within $5^{\circ}$. The two latter constraints ruled out the option to center on the HDF-S. To avoid the presence of a galaxy cluster, the field should not contain strong radio or $\mathrm{X}$-ray sources. The field which was chosen is located near the south galactic pole at $1^{\mathrm{h}} 6^{\mathrm{m}} 3.6^{\mathrm{s}}$, $-25^{\circ} 45^{\prime} 46^{\prime \prime}$ (J2000).

Approx. 10 GTO nights were eventually used for the FDF imaging in the optical, the rest of the allocated GTO for the FDF project was shared between several follow-up studies (e.g., the TF project or a study of high-redshift galaxies, see Mehlert et al. 2002, Noll et al. 2003).

The FDF imaging in $U, B, g, R$ and $I$ with FORS1+2 was performed between August 1999 and August 2000. Additional NIR observations in the $J$ and $K s$ bands with the New Technology Telescope on La Silla were gained in October 1999. The final photometric catalogue which is available to the public comprised 8753 objects and was described in Heidt et al. (2003). With a FOV of the coadded images of $\sim 40 \mathrm{arcmin}^{2}$, the FDF is approx. 8 times larger than the HDF-N.

Roughly three nights of dark time from the GTO pool were allocated to the TF project in total.
After a pilot observation in December 1999, the majority of the spectroscopy was performed in September and October 2000, followed by additional observations in October 2001. In the next two sections, the instrumental setups and selection techniques which are common between all observing runs will be described, whereas sections 2.4 to 2.6 will focus on the specific target sample characteristics and the observing conditions of the respective runs.

Roughly $30 \%$ of the observing time for the TF project were used for the construction of a distant field elliptical sample with $N_{\text {obj }} \approx 30$ within the FDF. For the sake of an effective filling of the MOS masks, these galaxies were observed simultaneously with the spirals. Since the kinematic analysis of early-type galaxies is, in contrast to spirals, based on absorption lines, the $\mathrm{S} / \mathrm{N}$ intended had to be 10 or higher (cf. next section). Therefore, faint early-types with apparent magnitudes $R>20.5^{m}$ were included in more than one setup and the individual spectra combined after data reduction. The data of these E/S0 galaxies were reduced by A. Böhm, B. Ziegler and A. Fritz. Some of the selection criteria for this subsample will be given below in brief, whereas the analysis of these objects is not the subject of this thesis and will be described elsewhere (Ziegler et al. 2003).

\subsection{Instrument Configuration}

As the aim of the project was the construction of a large spectroscopic sample within reasonable observing time, the Multi Object Spectroscopy Mode (MOS) was the best option. The Mask eXchangeable Unit (MXU) mode of FORS2 could not be used since it was not operationable in 2000 and the FDF observations in 2001 were restricted to the usage of FORS1. In MOS mode, 19 individually movable slits are used, while in MXU configuration, a mask with slits is cut prior to the spectroscopy.

For the configuration of the CCDs and the col- 
limator, the default values of the MOS mode were used, i.e. FORS was operated at standard resolution $(0.2 \mathrm{arcsec} / \mathrm{pixel})$ with a gain of $\sim 3 e^{-} / \mathrm{ADU}$ and the CCDs were read out in one-port mode. The quicker 4 -port readout mode ( $\sim 25 \mathrm{~s}$ vs. $\sim 92 \mathrm{~s}$ using one port) is appropriate mainly for direct imaging.

Both versions of FORS offer 19 slitlets in the MOS configuration, of which 9 have lengths of 22 arcsec, 8 have a lengths of 20 arcsec and the uppermost and lowermost slits have lengths of $\sim 11$ arcsec.

A variety of grisms is offered for spectrocopy with FORS. To achieve a medium resolution within the usable wavelength range appropriate for spirals at redshift $z \leq 1.0$, the grism $600 \mathrm{R}$ is optimal. For a slit position in the CCD center, a range $5200 \AA \leq \lambda \leq 7400 \AA$ is covered (see section 2.3.4 for a description of the correlation between slit position and wavelength window). The defaulted order separation filter GG435+81 was used.

Two other grisms with neighboring wavelength ranges were ruled out: Grism 600B had an upper wavelength limit that was too low $\left(\lambda_{\text {end }}=5900 \AA\right.$ in centered position $)$ to ensure the visibility of the [O II] doublet for highly redshifted spirals, whereas grism 600I reached into the near infra-red regime $\left(\lambda_{\text {start }}=6900 \AA\right.$ in centered position), i.e., the resulting spectra would have been heavily contaminated by atmosperic emission like, e.g., $\mathrm{OH}^{-}$-bands.

The slits could be set individually to widths between 0.3 arcsec and 60 arcsec. A fixed value of one arcsecond was chosen for all observations, yielding a resolution of $\mathrm{R} \approx 1200$ with the $600 \mathrm{R}$ grism. On the one hand, a smaller width would have increased the instrumental resolution, but on the other hand, a significant loss of flux then would have occured for seeing conditions above $\mathrm{FWHM} \approx 0.8$ arcsec, which roughly corresponds to the median at Paranal since 1998 according to the Differential Image Motion Monitor (DIMM). Based on the photometric redshifts catalogue, it was estimated that the majority $(\sim 95 \%)$ of the FDF spirals with $z \leq 1.0$ have an apparent brightness $R \leq 23^{m}$. Using the Exposure Time Calculator, an on-line tool maintained by ESO (www . eso.org/observing/etc/), an integration time of $\sim 2.5 \mathrm{hrs}$ was calculated to achieve an $\mathrm{S} / \mathrm{N} \geq 5$ in the emission lines for a typical spiral of brightness $R \approx 23^{m}$ with the configuration given above.

The pre-selection of the targets for spectroscopy was done within the ESO-MIDAS (Munich Image Data Analysis System) environment, whereas the final construction of the setups was performed with the FORS Instrumental Mask Simulator (FIMS). Catalogues with positions, magnitudes, structural parameters etc. of the target galaxies were created within MIDAS and used as input in the filling of the masks with the FIMS tool.

The output of the FIMS package consisted of three files per mask, defining, e.g., the position and orientation of the mask in the plane of the sky, coordinates of reference stars in the fieldof-view (for the positioning of the mask in the observations with an accuracy $<0.1$ arcsec), the positions of the individual slits etc. These output files in turn were used for the construction of the so-called Observing Blocks which act as input to the operating system of the telescope in the execution of the observations. For a more extensive description of the operation environment, see the "FORS1+2 User Manual" released by ESO.

\subsection{Basic Selection Criteria}

The input catalogues for the construction of the MOS setups were extracted from the photometric redshifts catalogue of the FDF. Since most of the direct imaging was performed from $\mathrm{Au}-$ gust to December 1999 and got reduced between January and May 2000 by three members of the FDF consortium (A. Böhm, J. Heidt and K. Jäger), the first catalogue with photometric redshifts which benefitted from the full data set 
became available in August 2000. Therefore, it was possible to use the deep catalogue firstly in the target selection process of the observations in September 2000, whereas the preparation of the single setup observed in December 1999 was restricted to a preliminary catalogue from October 1999. Targets for the MOS observations were pre-selected from these catalogues according to a combination of parameters which will be described in the following.

\subsubsection{Apparent Brightness}

Basically, the apparent brightness was the only selection criterion. All other parameters taken into account (following sections) were used solely to ensure the visibility of emission lines or to avoid face-on spirals or large geometric distortions. For the TF spirals, this limit in total magnitude was $R \leq 23^{m}$ as derived with the Mag_auto algorithm of the Source Extractor package (Bertin \& Arnouts 1996). Since a higher $\mathrm{S} / \mathrm{N}$ ratio was mandatory for the galaxies in the early-type sample, the limit was set to $R \leq 22^{m}$ for these objects.

\subsubsection{Spectrophotometric Type}

In the August 2000 release of the FDF photometric redshifts catalogue, estimated redshifts and spectrophotometric types of more than 3800 objects were given (Bender et al. 2001). A parameter called model represented the best-fitting Spectral Energy Distribution (SED) type of the respective objects. Each integer value of this parameter between 1 and 12 corresponded to a certain star formation rate e-folding time of the best-fitting SED template. Thanks to this information, it was possible to discriminate between late-type and early-type galaxies. Spirals were characterised by values model $\geq 3$, while E/S0 galaxies had values model $=1$ or $\operatorname{model}=2$.

Catalogue entries featuring model $=0$ could not be fitted properly via the photometric redshifts technique, but this was the case only for $11 \mathrm{ob}-$ jects with $R \leq 23^{m}$.

\subsubsection{Structural Parameters}

Contrary to the three other parameters $R$, model and $z_{\text {phot }}$, the position angles were not taken from the photometric redshifts catalogue. Instead, these and the inclination angles were measured in a coadded $I$-band frame of the 10 best-resolved FDF images, to ensure the highest possible accuracy. This frame had a PSF of 0.49 arcsec FWHM and a total integration time of $3000 \mathrm{~s}$.

Using the Source Extractor package, four parameters were derived for each $2 \sigma+$ detection in the reference frame. These were the apparent major and minor axes, $a$ and $b$, the position angle $\theta$ and the so-called star classification parameter, star. The latter represents the propability for a certain detection to be a point source or extended.

Inclinations (angle between the normal vector of the disk and the line-of-sight) were computed using the combined values of the apparent major and minor axes via (Heidmann et al. 1971)

$$
\left.\cos ^{2} i=\left((a / b)^{2}-q^{2}\right)\right) /\left(1-q^{2}\right) .
$$

Here, the factor $q$ is the assumed axial ratio for edge-on systems, in other words, the ratio between disk scale length and scale height. This factor $q$ was fixed to 0.2 , which is the observed value for typical spirals (e.g., Tully et al. 1998). Since one of the aims of the project was the derivation of spatially resolved rotation curves, face-on spirals (i.e. spirals with low inclinations) had to be rejected from the target catalogue. Face-on disks are observed perpendicular to their plane of rotation and no Doppler shifts can be measured in that case. Based on earlier studies of distant disk galaxies like, e.g., Vogt et al. $(1996,1997)$, the lower limit on the inclination was set to $i=40^{\circ}$.

The position angle $\theta$ denotes the orientation of the apparent major axis in the plane of the sky. 
Throughout this thesis, the convention will be that $\theta$ gives the angle between the apparent major axis and the horizontal axis, counted counterclockwise. To minimize the geometric distortions which arise from deviations (denoted by an angle $\delta$ ) between the major axis and the slit direction of a certain spiral, the target catalogues were split into subsets according to the position angles with a binning of $30^{\circ}$, i.e. the constraint to the misalignment was $\delta \leq 15^{\circ}$. In this manner, the complete range of orientations could be covered with six MOS masks which are rotated by modulo $30^{\circ}$.

E/S0 galaxies are, contrary to spirals, dynamically hot, i.e. the velocity dispersion exceeds the rotation velocity at all radii (e.g., Burstein et al. 1997). Moreover, these objects are mostly triaxial. For the MOS targets with early-type SEDs, neither inclinations nor position angles have therefore been taken into account as selection criteria. But, since a small fraction of earlytype galaxies in the photometric redshifts catalogue could be misclassified dwarf stars (Saglia 1999, priv. com.), it was mandatory to use structural parameters for a robust star/galaxy separation. Source Extractor detections which most probably were bonafide stellar objects featured star $\geq 0.9$ and $a \approx b \approx 2.5$ arcsec. Therefore, targets with star $\geq 0.9$ or $a \approx b \leq 3$ arcsec were rejected.

\subsubsection{Photometric Redshift}

Additionally to the SED parameter model, the photometric redshifts catalogue comprised the best-fitting photometric redshift $z_{\text {phot, }}$ its uncertainty $d z_{\text {phot }}$, the second best-fitting photometric redshift $z_{\text {phot2 }}$ and the probability $P$ that the latter was correct. According to the SED and the position of the respective targets within the FDF (or, more precisely, within the coordinate system of the MOS mask), these redshifts were taken into account in the target selection process to ensure the visibility of certain lines in the spectra. These lines will be specified further below.

An effect that has to be considered here is the correlation between the position of a slit and the wavelength range in the resulting spectrum. As has been stated before, for a slit that is centered on the X axis of the MOS mask, the starting and ending wavelengths with the grism R600 correspond to $\lambda_{\text {start }} \approx 5200 \AA$ and $\lambda_{\text {end }} \approx 7400 \AA$, respectively. However, the starting wavelength $\lambda_{\text {start }}$ and the ending wavelength $\lambda_{\text {end }}$ depend on the position of the slit on the $\mathrm{X}$ axis. If a slit is shifted to the extreme left hand side of the MOS mask, the covered wavelength range will be approx. $6300 \AA \leq \lambda \leq 8500 \AA$. On the other hand, for a slit position at the extreme right hand side of the mask, the range will be approx. $4100 \AA \leq \lambda \leq 6300 \AA$.

In the case of the target spirals, it was aimed to either cover the $[\mathrm{O}$ II] 3727 doublet, the $\mathrm{H} \beta$ or the [O III] 5007 emission lines with the spectra. $\mathrm{H} \alpha$ emission would be redshifted out of the range of the grism $600 \mathrm{R}$ at $z \approx 0.25$ even if the slit was positioned extremely on the left hand side of the mask. To gain at least the [O II] emission in the spectra, a few spirals with $R \leq 23$ and $z_{\text {phot }}>1.2$ were excluded from the catalogue. Moreover, it was checked for each spiral which was eventually selected for spectroscopy that the [O II] doublet would not exceed the wavelength range for the respective slit position.

For the early-type targets, a limit of $z \leq 0.6$ was chosen in order to include the $\mathrm{Mg}_{b}$ index at $5170 \AA$ in the spectra (for a study of the $\mathrm{Mg}-\sigma$ relation, see, e.g., Dressler et al. 1987). As an additional star/galaxy separation criterion, targets with insignificant photometric resdhift, i.e., $z_{\text {phot }}-d z_{\text {phot }} \leq 0$, were rejected.

\subsection{9: A Pilot Observation}

A first MOS setup was scheduled for observation with FORS1 in December 1999. Since at that time the full FDF imaging data was not yet reduced, the target selection had to be based 
on a coadded $1200 \mathrm{~s} R$-band frame with a PSF of 0.72 arcsec FWHM from the commissioning phase of FORS1. An even larger shortcoming than this limited resolution was that the selection process had to rely on a preliminary version of the photometric redshifts catalogue. Therefore, the constraints on inclination and slit misalignment had to be relaxed substantially to fill up the mask.

All slits were positioned exactly on the optical center of the galaxies. For this, the display cuts (the lower and upper limits for the color table) of the FIMS software were individually adjusted to display only the innermost, brightest regions. The same method was used for the selections in 2000 and 2001.

13 spirals, 3 early-type galaxies and 3 objects exceeding the FOV of the catalogue, i.e. galaxies without information on the SED or $z_{\text {phot }}$, were selected. This was inevitable for the slits on the edges of the mask (slits \#1, \#18 and \#19) since the catalogue only covered $\sim 6 \times 6$ sq.arcmin, whereas the MOS mask spanned $\sim 6.8 \times 6.8$ sq.arcmin. Based on the spectra of the three "anonymous" objects with respective $R$-band brightnesses of $19.1^{m}, 19.4^{m}$ and $20.8^{m}$, it turned out that they were earlytype galaxies.

The spectroscopy was split up into four exposures of $2250 \mathrm{~s}$ each to limit the number of cosmic ray events per frame. Furthermore, the individual frames could be compared to indentify the cosmics. During the observations, which were performed in Visitor Mode by C. Möllenhoff (Heidelberg) and K. Reinsch (Göttingen), the mean DIMM seeing was 0.66 arcsec.

This pilot oberservation eventually yielded only four rotation curves which could be used to derive the maximum rotation velocity. Nevertheless, it was valueable for the obervations to come since it could be observationally confirmed that an inclination of $40^{\circ}$ indeed was a reliable lower limit: Of the 9 spirals which were not appropriate for a derivation of $V_{\max }$, only two had $i>40^{\circ}$. One of these was a faint, early-type spiral (SED type Sb) with extremely low S/N of the emission lines, the other was a very late-type spiral (type Sdm) with a rotation curve showing strong kinematic perturbations.

\subsection{Observations in 2000}

In September and October 2000, two nights of FDF GTO time were allocted in total to the TF project. Thus, six MOS setups could be observed with FORS2 at an integration time of $2.5 \mathrm{hrs}$ each. Fortunately, the photometric catalogue based on the full imaging was available, and the reference frame with a PSF of 0.49 arcsec FWHM could be used in the preparation of the masks.

To achieve an effective filling of the MOS setups, the targets from the photometric redshifts catalogue were sub-divided in the following respects. Firstly, the spirals were grouped into subsets according to their position angles with a binning of $30^{\circ}$. This way, all orientations could be covered by one of the six MOS masks and any possible misalignment was limited to $15^{\circ}$.

Then, each set was splitted into three categories. Spirals which met all of the constraints described in section 2.3 were placed in the catalogue with the highest priority. Late-type galaxies which had an inclination in the range $30^{\circ} \leq i<40^{\circ}$ were put in a second catalogue. This relaxation of the lower limit was motivated by the difference between the inclinations computed via Eq. 2.1 with the apparent axis ratios derived using the Source Extractor and the inclinations determined by surface brightness profile fits (see chapter 4.3). Since the PSF, which tends to make a galaxies isophotes more round, could be accounted for only with the latter method, Extractor-based inclinations were on the mean $\sim 7^{\circ}$ lower than those measured with profile fits. However, it was not practical to perform these time-consuming fits for all target spirals as standard. Instead, the slightly reduced lower limit 
was used as given above.

As a third category, spirals which slightly exceeded the apparent brightness limit $\left(23^{m}<R \leq 23.3^{m}\right)$ were placed in separate catalogues. These were considered to have the lowest priority and were only used as "fill-up objects" if no other target galaxy was available for a certain MOS slit.

In this manner, three target spiral catalogues per mask, 18 in total, were constructed, comprising 70 galaxies of the highest priority, 35 within the relaxed inclination limits and a further 15 which fulfilled the relaxed brightness limit. Additionally, a list of 22 early-type targets was generated which was not sub-divided according to the structural parameters for the reasons outlined at the end of section 2.3.3.

The relatively small number of target spirals mainly was due to the small FOV of the deep photometric catalogue which corresponded to approx. $5.5 \times 5.5 \operatorname{arcmin}^{2}$. Therefore, the older versions of the catalogue which covered a larger fraction of the FDF were used additionally in the selection process to gain data on some galaxies in the field edges.

For the filling of each mask, the three spiral catalogues of the respective position angle range and the early-type catalogue were loaded into the FIMS software simultaneously and marked with different symbols. In this way, the final object selection could be performed timeefficiently. For the slits at the mask edges, however, the coverage of the catalogues was small or even null. Therefore, anonymous objects had to be selected in some cases (see below) as for the pilot study. Moreover, even in the inner regions of some masks a slit was devoid both of target spirals with the appropriate position angles and of early-type candidates. In these cases, either spirals with neighboring positions angles or bright anonymous objects from the outer FDF regions were selected.

Besides the correlation between slit position and wavelength range, the position of a target within the slit itself had to be considered. In the direction of dispersion, this was achieved by individually adjusting the cutvalues to display only the brightest, innermost regions of each galaxy. Combined with a magnified display of the respective object, the slits could be positioned on the optical center very precisely this way.

Basically, a centered position within the spatial direction of a would be desireable, because then the night sky emission could be determined very robustly from both sides of the target. An object's position along the spatial axis, however, was fixed by its coordinates within the FDF and the position and rotation angle of the mask, since all MOS slits could only be adjusted in the direction of the $\mathrm{X}$ (dispersion) axis. Therefore, both the exact rotation angle and the central position of a MOS mask were chosen carefully prior to the setting of the individual slits. This procedure benefitted from the simultaneous display of the four target catalogues also. In the case of one particular setup (covering the position angles $0^{\circ} \leq \theta \leq+30^{\circ}$ which corresponds to a "straightforward" mask rotation angle of $\alpha=-75^{\circ}$ ), the mask was rotated by an additional $0.3^{\circ}$ to achieve a better coverage of the high-priority targets. This mask was furthermore rotated by $180^{\circ}$, i.e. the final rotation angle corresponded to $\alpha=-104.7^{\circ}$, to adjust the observeable wavelength range to the photometric redshifts distribution of the targets.

Nevertheless, in each setup some galaxies had to be selected in spite of their position near the slit edges. This particular shortcoming of the MOS configuration would not have been present in MXU mode, where each slitlet has two spatial degrees of freedom but, as already stated, the latter mode was no option for the TF project spectroscopy. A complete MOS setup is shown in Fig. 2.1, and the positioning of an individual slit on a target spiral is illustrated by a magnified extraction of the same setup in Fig. 2.2.

An overview of the final 6 MOS setups is presented in Table 2.1. In total, 71 spirals, 28 early- 


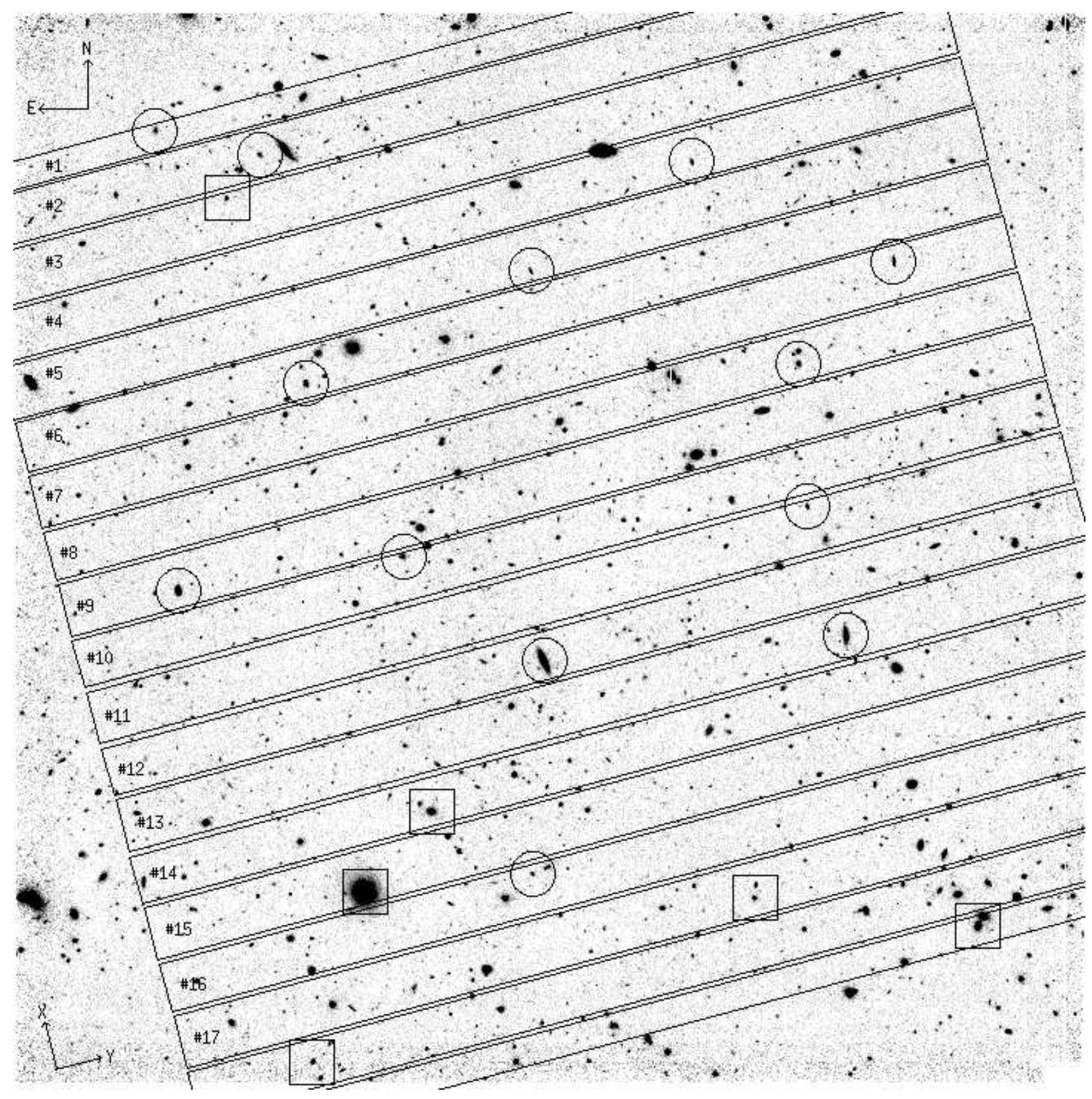

Figure 2.1: FORS2 MOS setup from the observations in 2000. The mask was rotated by $\alpha=+15^{\circ}$ to cover position angles between $-60^{\circ}$ and $-90^{\circ}$. North is up and east to the left, slit labels are given for \#1 to \#17. The small coordinate sytem in the lower left corner denotes the two axes in the resulting spectra, i.e. the direction of dispersion (X axis) and the spatial axis $(\mathrm{Y})$. Each rectangle indicates the physical region of the respective slit. For matters of clarity, the slitlets are not shown, see Fig. 2.2 for a visualization. The targets which were selected are indicated by circles (spirals) and squares (ellipticals), respectively. In some cases, in was inevitable to select galaxies which are located very close to the slit edge (slits \#1, \#3, \#10 and \#11). The displayed field-of-view corresponds to $7.22 \times 7.22 \mathrm{sq} . \operatorname{arcmin}$. 


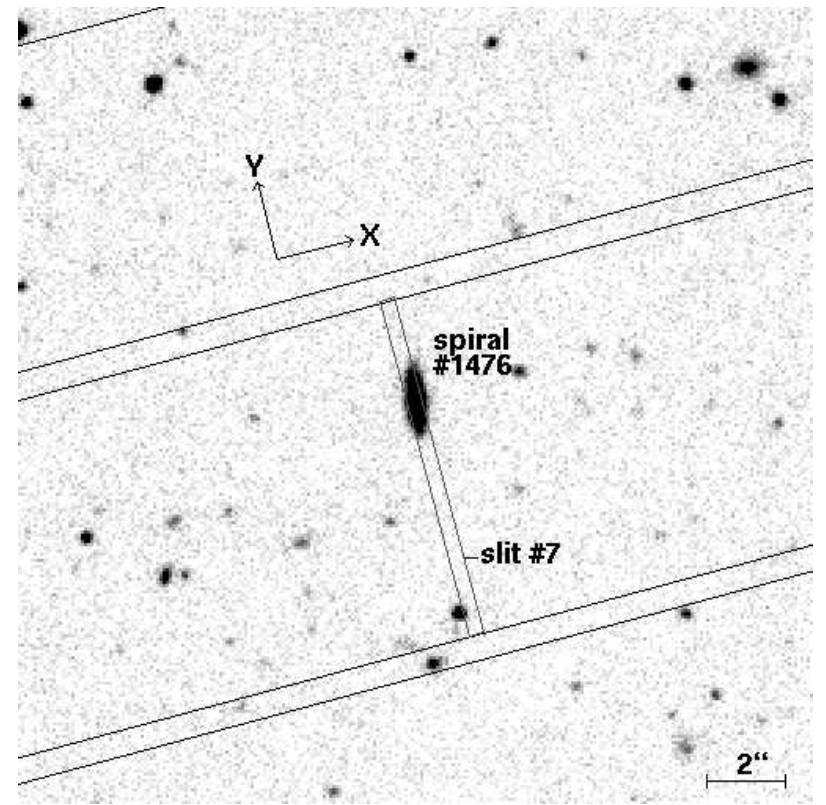

Figure 2.2: Magnification of Fig. 2.1 showing the region of slit \#7 centered on galaxy \#1476, a spiral of inclination $i=77^{\circ}$ at a position angle $\theta=-85^{\circ}$. Since the position angle of the slit is $\theta=-75^{\circ}$, corresponding to a rotation angle of the MOS mask of $\alpha=+15^{\circ}$, the misalignment angle of the spiral is $\delta=-10^{\circ}$. The object number corresponds to the galaxies ID in the FDF photometric catalogue presented in Heidt et al. (2003) and also to the IDs in the full data table in the appendix. Note that the apparent slight off-center position of the object is just due to the resolution, this image was created with the MIDAS package and does not show the original FIMS configuration.

type and 6 anonymous galaxies were observed. Note that these values are corrected for double hits: One spiral and eight early-type galaxies have been observed twice. In the case of the former, this was just due to the lack of alternatives for the filling of the certain slit, whereas for the latter, it was done to increase the $\mathrm{S} / \mathrm{N}$ of relatively faint ellipticals with $R \geq 20.5^{m}$.

Furthermore, the number of 71 spirals includes three galaxies which were not comprised by any version of the photometric redshifts catalogue but nevertheless clearly showed a late-type morphology with spiral arms. Two of the $28 \mathrm{E} / \mathrm{S} 0$ galaxies already were observed in 1999 .

Table 2.1: Overview of the selected targets in 2000. The columns give the covered range of position angles, the number of spirals, E/S0 galaxies and anonymous galaxies included in the individual MOS setups. Note that galaxies which were observed twice are counted only once for the total number of objects.

\begin{tabular}{|c|c|c|c|}
\hline$\theta$ range & Spirals & $\mathrm{E} / \mathrm{S} 0$ & anon \\
\hline $\begin{array}{r}-90^{\circ} /-60^{\circ} \\
-60^{\circ} /-30^{\circ} \\
-30^{\circ} \% 0^{\circ} \\
0^{\circ} /+30^{\circ} \\
+30^{\circ} /+60^{\circ} \\
+60^{\circ} /+90^{\circ}\end{array}$ & $\begin{array}{l}13 \\
14 \\
12 \\
12 \\
11 \\
10\end{array}$ & $\begin{array}{l}5 \\
5 \\
6 \\
5 \\
8 \\
7\end{array}$ & $\begin{array}{l}1 \\
- \\
1 \\
2 \\
- \\
2\end{array}$ \\
\hline total & 71 & 28 & 6 \\
\hline
\end{tabular}

Two of the MOS setups were observed by S. Noll (Heidelberg) in September 2000, the remaining four were observed by D. Mehlert (Heidelberg) in October 2000. In table 2.2, some of the observing conditions are listed. Each MOS setup was split into three exposure of $3000 \mathrm{~s}$, yielding a total integration time of $2.5 \mathrm{hrs}$. The seeing conditions ranged from good to excellent $\left(\mathrm{FWHM}<0.5^{\prime \prime}\right)$ and shortly raised above 1.0 arcsec only once during the observations of the fourth mask. To limit the loss of flux due to atmospheric absorption, the constraint on the airmass was set to $A \leq 2.0$.

Bias, dark, flatfield and wavelength calibration frames were taken by the ESO staff in the daytime.

\subsection{Observations in 2001}

In the beginning of 2001, an additional amount of GTO time was granted by ESO for the FDF 
Table 2.2: Overview of the observing conditions in 2000. The columns give the range of position angles, the observation date, the mean airmass and the mean DIMM seeing value of the six MOS masks.

\begin{tabular}{rrrl}
\hline \multicolumn{1}{c}{$\theta$ range } & \multicolumn{1}{c}{ date } & airm. & DIMM \\
& & & \\
\hline$-90^{\circ} /-60^{\circ}$ & $9 / 27 / 00$ & 1.21 & $0.51^{\prime \prime}$ \\
$-60^{\circ} /-30^{\circ}$ & $9 / 27 / 00$ & 1.33 & $0.43^{\prime \prime}$ \\
$-30^{\circ} / 0^{\circ}$ & $10 / 04 / 00$ & 1.40 & $0.81^{\prime \prime}$ \\
$0^{\circ} /+30^{\circ}$ & $10 / 05 / 00$ & 1.36 & $0.80^{\prime \prime}$ \\
$+30^{\circ} /+60^{\circ}$ & $10 / 05 / 00$ & 1.28 & $0.74^{\prime \prime}$ \\
$+60^{\circ} /+90^{\circ}$ & $10 / 06 / 00$ & 1.15 & $0.66^{\prime \prime}$ \\
& & & \\
\hline
\end{tabular}

collaboration. ESO decided to do so to compensate the significant time loss during the FDF imaging phases which had been caused by the El Niño phenomenon. The partly very bad seeing in the second half of 1999 had led to the necessity to redo a substantial fraction of the observations. One night of dark time from the new GTO pool was thankworthy allocated to the TF project by the P.I. of the FDF collaboration, Prof. I. Appenzeller (Heidelberg). Since the spectroscopy was scheduled to be performed in October 2001, this night corresponded to over eight hours of spectroscopy at an airmass $A \leq 2$, or to three MOS setups including the overhead time. The MXU mode was not available since the complete GTO pool was restricted by ESO to the usage of FORS1.

Indeed it was decided to keep the total integration time per setup unchanged, instead of, e.g., using the whole amount of observing time for just one or two masks. Nevertheless, since the vast majority of the high-priority $\mathrm{TF}$ targets had been observed already, it was clear that the follow-up spectroscopy was to be focussed on galaxies of either relatively low inclinations or faint brightnesses.

Since the reference $I$-band image which had been used in the preparation of the previous observations was a co-addition of FORS2 frames, it was not appropriate for the construction of the new MOS setups with FIMS. This was due to a small but nevertheless non-negligible difference in the spatial scales of the two FORS instruments which amounted to approx. 10 pixels over the total FOV. Moreover, a comparison between the positions of FDF stars in a FORS1 and a FORS2 frame revealed that the world coordinate systems were very slightly rotated against each other. Therefore, a second reference frame was constructed using the 10 best-resolved FORS1 $I$-band images with the same procedure as the FORS2 reference frame. The final image featured a PSF of 0.52 arcsec FWHM. Pixel and world coordinates in all catalogues then were transformed into the FORS1 coordiante system.

As a first step in the selection process, all late-type galaxies from either of the photometric redshifts catalogues fulfilling the constraints $R \leq 23.3^{m}, i \geq 24^{\circ}$ and $z_{\text {phot }}<1.2$ were merged into a single table. All targets which had been observed in 1999 or in 2000 were rejected. The considerable relaxation of the lower inclination limit was chosen to ensure that no spiral would be de-selected due to an underestimated inclination. Since an effective lower limit of $30^{\circ}$ was aimed for, a decrease to $24^{\circ}$ was chosen to compensate for the mean offset between the Source Extractor-based inclinations and the time-expensive surface brightness profile fits which took the seeing into account (see chapter 4.3).

Following this, all candidate objects were visually inspected to check their inclinations and position angles which were initially based on the Source Extractor output. For roughly half of the galaxies (mostly very faint objects), the inclinations or the position angles were considered to be inaccurate and surface brightness profile fits were performed to derive the exact structural parameters. In particular, all Extractorbased inclinations $i<30^{\circ}$ were verified. A limit 
of $i \geq 30^{\circ}$ to the confirmed values then yielded a target sample of 46 late-type galaxies.

It was to be expected that the constraint on the misalignment angles would have to be relaxed as well to gain a sufficient number of targets per MOS setup. However, the distribution of the position angles in the target sample revealed that this was not necessary: 35 of the 46 targets featured a position angle $\theta \geq 0^{\circ}$ and were distributed uniformly enough to yield at least 10 spiral targets within bins of $30^{\circ}$.

To put together a new target sample of ellipticals, the photometric redshifts catalogues were searched for early-type galaxies with $R \leq 22^{m}$ and $z_{\text {phot }}<0.6$ which had not yet been observed. In this search, objects from the southwestern corner of the FDF were neglected to avoid the outskirts of the cluster at $z=0.33$ (see chapters 4.1 and 6.4.3 for a discussion of this topic). 15 out of the 29 ellipticals with determined redshifts from the previous observations were probably cluster members, and it was the aim of the follow-up spectrocopy to only consider ellipticals in the field. However, after neglecting potential stellar objects, only two "new" targets remained. Eight bonafide field ellipticals with $R>20.5^{m}$ which already had been observed were additionally selected to increase the total integration time and thus the $\mathrm{S} / \mathrm{N}$ of the combined spectra.

39 spirals (of which one was already observed in 2000), 10 early-type and four unclassified galaxies were eventually selected for the spectroscopy. The given number of spirals includes seven objects exceeding the FOV of the target catalogues which showed a late-type morphology. Except one bright elliptical with $R=19.0^{m}$, all earlytype targets were observed two or three times including the spectroscopy in 1999/2000.

The limited number of spirals which were not covered by any of the previous MOS setups had an imprint on the apparent brightness distribution of the new target sample. In 2000, the median of all late-type galaxies was $\langle R\rangle \approx 21.8^{m}$, whereas the spirals selected in 2001 featured a value of $\langle R\rangle \approx 22.8^{m}$.

Along with a range of other FDF projects, the observations were carried out in Visitor Mode by J. Heidt (Heidelberg) in October 2001. The MOS setups again were splitted into three exposures with a total integration time of $9000 \mathrm{~s}$. An outline of the observing conditions is given in table 2.3. The seeing was slightly poorer than during Sep./Oct. 2000, but still in the subarcsecond regime.

Table 2.3: Overview of the observing conditions in 2001. The columns give the range of position angles, the observation date, the mean airmass and the mean DIMM seeing value of the three MOS masks.

\begin{tabular}{rccc}
\hline$\theta$ range & date & airm. & DIMM \\
\hline $0^{\circ} /+30^{\circ}$ & $10 / 12 / 01$ & 1.43 & $0.76^{\prime \prime}$ \\
$+30^{\circ} /+60^{\circ}$ & $10 / 14 / 01$ & 1.07 & $0.89^{\prime \prime}$ \\
$+60^{\circ} /+90^{\circ}$ & $10 / 12 / 01$ & 1.38 & $0.82^{\prime \prime}$ \\
\hline
\end{tabular}




\section{Chapter 3}

\section{Data Reduction}

The reduction of the optical multi-band imaging of the FDF, from which the apparent magnitudes of the TF spirals were derived, was performed mainly by five members of the FDF consortium (A. Böhm, J. Heidt, K. Jäger in 2000/2001, A. Gabasch und J. Heidt in 2002). A preliminary reduction of imaging data taken during the commissioning phase of FORS had been done in 1999 by J. Heidt and K. Jäger. The reduction of this large amount of data is described elsewhere (Heidt et al. 2003) and was not part of this thesis. Here, only the reduction steps of the medium-resolved spectroscopy for the TF project will be given. The only exception is the $I$-band reference frame which was especially created for the derivation of the structural parameters and the morphology of the TF spirals with the highest resolution which was achieved during the FDF imaging. For a description of how this image was constructed, see section 3.7.

The reduction procedure was implemented using the ESO-MIDAS (Munich Image Data Analysis System) environment. A variety of algorithms for data processing is provided within this package for applications like the emission line identification, wavelength calibration and rebinning of two-dimensional spectra. Nevertheless, special routines had to be developed in the MIDAS command language for a proper correction of the raw VLT spectra like, e.g., the distortions of the focal reducer (see section 3.4).

All the following sections will focus on the special aspects of VLT spectra reduc- tion. A theoretical review of data reduction will not be given here, see, e.g., Wagner (1992) or Volume B of the ESO-MIDAS User's Guide, Chapter 6 (available for download at www. eso.org/projects/esomidas/doc/). Furthermore, only the nine MOS setups which were observed in 2000 and 2001 will be considered. Since the pilot observation of a single mask with FORS1 in 1999 (see chapter 2.4) eventually yielded rotation curves of only four spirals, the reduction of these data will not be described here separately.

The individual stages of the reduction procedure will be discussed in the same order as they were applied to the data.

\subsection{Bias}

For all observing runs, the bias sets taken in oneport readout mode with low gain at the beginning and the end of the nights showed a very stable spatial variation of $\leq 2 \mathrm{ADU}$ at a median of $\sim 226$ ADU. This two-dimensional structure was time-independant to within $<0.1$ ADU. Therefore, all bias frames from the nights of the 2000 and 2001 observations were used to generate two masterbiases. This was done by additively normalizing the individual frames to the same median count rate, followed by a median-average of the two sets. In the histogram of the resulting FORS2 bias, 12 hot pixels were identified and replaced by the median. For a further decrease of the noise, the averaged biases 
were slightly smoothed using a Gaussian filter with $\sigma_{x, y}=3$ pixel which yielded a r.m.s. of $\sim 0.5 \mathrm{ADU}$ in both masterbiases.

In the median, the count rates of pre- and overscan were $\sim 1$ ADU below those in the physical $\mathrm{CCD}$ region of the bias frames. When the masterbiases were subtracted from the individual science, flatfield and wavelength calibration frames, this slight offset value was taken into account. Except for the bias frames, all images featured a difference of $\sim 0.4$ ADU between over- and prescan. Therefore, correction factors that had to be applied beyond the bias subtraction were derived via the overscan difference in masterbias and object frame.

Since the dark current was very uniform across the CCDs and amounted to less then 2 ADU per hour, an extra dark frame subtraction has been neglected.

\subsection{Cosmics}

Although the observation of each MOS setup was split into three exposures, a cosmic ray removal by a simple median-averaging of the frames was no option because the CCD positions of the individual galaxy spectra were not time-independant. If the zenithal distance was significantly varying during the observations of a mask, the spatial position of a spectrum changed by up to two pixel $(0.4$ arcsec) between the first and last exposure. This was also the reason why every slit exposure was reduced seperately.

For a removal of a fraction of the cosmic ray events, the MIDAS algorithm FILTER/COSMIC was applied to the complete MOS frames. To avoid any damage to real emission features from the galaxies, the parameters of the filter algorithm which tune the discrimination between physical objects and artificial features were set to moderate values. Furthermore, each frame and the map of removed pixels were visually inspected after the filtering. A second modest cosmics filter was used at the stage of the sky subtraction (see section 3.6). However, the final correction was performed by a purely manual removal of any contaminated pixels, if artificial features were indentified in a visual inspection of the completely reduced $1 \mathrm{D}$-spectra or the spatially resolved emission lines that were used for rotation curve extraction.

\section{$3.3 \quad$ Flatfield}

Spectroscopy flatfield (FF) through-mask exposures with FORS are taken with two sets of lamps switched on alternately. With the default exposure times ( $150 \mathrm{sec}$ with FORS2 and 200 sec with FORS1), the spectra typically have $\sim 15000$ ADU. Since these calibration images are purley "internal" flats, they are not contaminated by cosmics. However, a shortcoming of the frames is that either the upper or lower CCD half are not usable due to contaminating light from the gaps between the slits. To overcome this problem, the upper and lower halfs have to be normalized and median-averaged seperately before they can be re-combined to a masterflat. The FORS2 spectra taken in 2000 showed 7 columns of non-linearly decreased sensitivity (so-called "bad columns") which could not be corrected via flat-fielding. Instead, they were set to the mean of the adjacent columns.

Previous to the following reduction steps, the slit regions of calibration and science frames were individually extracted and further reduced like long-slit data. Since every exposure was processed seperately, $3 \times 19=57$ science spectra per MOS setup and $9 \times 57=513$ science spectra in total were reduced.

The FF slit exposures were approximated by a polynomial fit of sixth degree in dispersion direction (X axis) to account for the CCD response function. These fits were used for the normalisation of the flats prior to the correction of the pixel-to-pixel variations.

For a small fraction of the science spectra, it turned out that the flat-fielding introduced arti- 
ficial tilts along the spatial axis. This may have been due to the large differences in the typical count rates of the science and the flatfield spectra which amounted to two orders of magnitude. As can be observed in the direct imaging modes (see section 3.7), the large--scale sensitivity of the FORS detectors is not perfectly linear from $\sim 10^{2}$ to $\sim 10^{4}$ ADU. An artificial tilt across the direction of dispersion, however, affects the accuracy of the most critical reduction step, the night sky subtraction. Therefore, spectra which showed a variation by more than a few percent along the $\mathrm{Y}$ axis after flat-fielding were kept without a correction of pixel-to-pixel variations.

\subsection{Rectification}

In the next step, the geometric distortions caused by the focal reducer were corrected. For the slits at the bottom and top of the CCD, where the distortions were maximal, the curvature of spectral features corresponded to a displacement of up to 5-6 pixel both in $\mathrm{X}$ and $\mathrm{Y}$ direction, whereas slit \#10 in the middle of the chip was undistorted. The curvature of raw MOS spectra is visualized in Figs. 3.1 and 3.2, note that the identification numbers of the slits increase from top to bottom by convention.

A polynomial fit of second degree was fitted to the galaxy spectra in each slit to derive the curvature along the spatial axis. Based on this fit, the science and wavelength calibration spectra were rectified. This was done by re-binning the respective spectra in $\mathrm{Y}$ direction to a scale of 0.02 arcsec and shifting each column according to the fit either in the $+\mathrm{Y}$ direction in the case of slits \#11 to \#19 or the $-\mathrm{Y}$ direction for the slits \#1 to \#9. Tests confirmed that this procedure was flux-conserving to within a few percent.

To check the accuracy of the distortion correction, one can compare the fits to a galaxy spectrum before and after rectification. E.g., in the case of slit \#2 of one of the FORS1 masks which

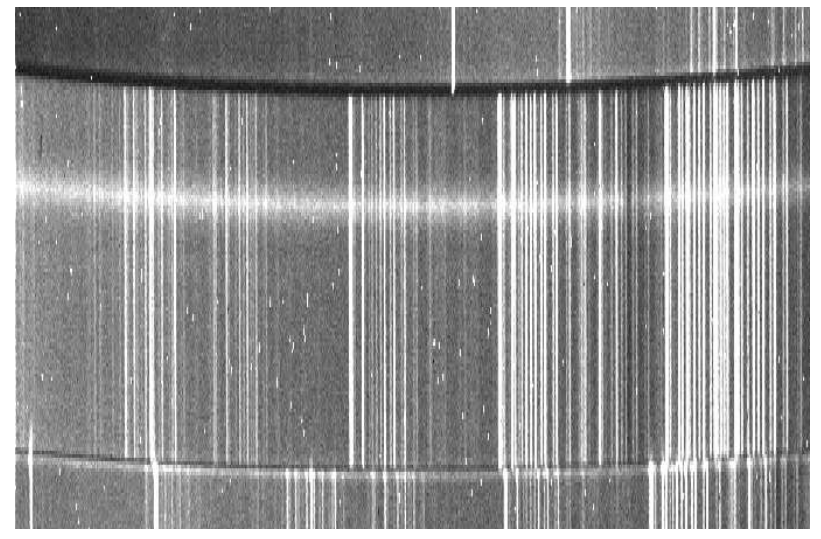

Figure 3.1: Upper part of a raw FORS1 MOS frame with 3000 seconds exposure time showing the region of slit \#2. Wavelength is increasing parallel to the $\mathrm{X}$ axis from left to right. A relatively bright $\left(R=19.94^{m}\right)$ galaxy spectrum is overlaying the night sky emission lines. Note that the magnification scale is larger along the $\mathrm{Y}$ axis to demonstrate the distortions by the focal reducer in this direction. The $\mathrm{X}$ axis corresponds to $\sim 2000$ pixel, the $\mathrm{Y}$ axis to $\sim 100$ pixel.

is displayed in Fig. 3.1, the scatter of the galaxy profile along the spatial axis was 1.59 pixel (corresponding to 0.32 arcsec) prior to rectification and 0.05 pixel (corresponding to 0.01 arcsec) afterwards.

The distortions along the $\mathrm{X}$ axis, i.e. the artificial tilts of the emission lines, were corrected during the wavelength calibration.

\subsection{Wavelength Calibration}

During ESO Periods 65 and 66 (i.e., during the observations in 2000), FORS wavelength calibration frames for grism R600 spectra were taken by default with $\mathrm{He}$, Ar and Ne lamps simultaneously switched on. The disadvantage of this combination of lamps is the almost total lack of lines below $5770 \AA$ : only two weak He emission features at $4713 \AA$ and $5015 \AA$ are present in this configuration. For slits which are positioned far to the right hand side of a MOS mask, i.e. for low starting wavelengths of the spectra, this in- 


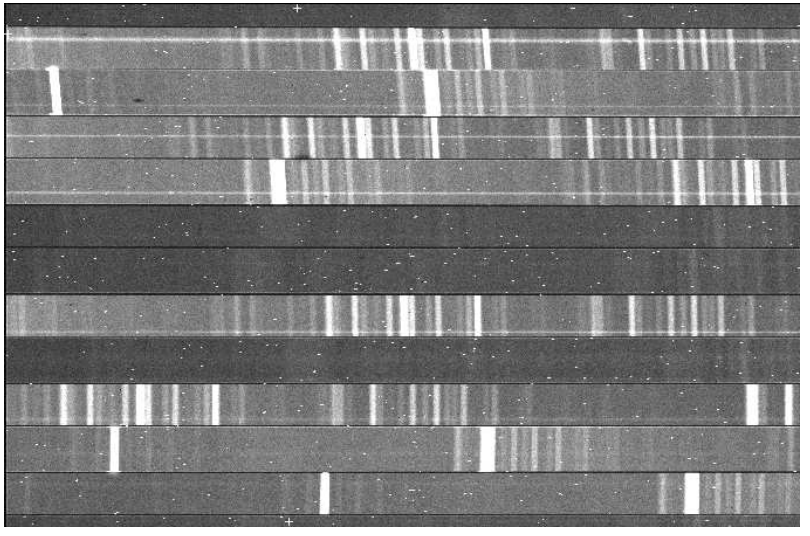

Figure 3.2: $\quad$ The same MOS frame as in Fig. 3.1 with a different magnification scale showing the slits $\# 1$ to $\# 12$. The $\mathrm{X}$ axis corresponds to $\sim 700$ pixel, the $\mathrm{Y}$ axis to $\sim 1300$ pixel. The curvature of the night sky emission lines in $\mathrm{X}$ direction is clearly visible in the uppermost slits, whereas slit \#10 in the center of the CCD is undistorted. Note that the apparent lack of night sky lines in slits \#1, \#6, \#7 and \#9 is just due to the displayed wavelength range. In the case of these four slits, the strong $5580 \AA$ feature is shifted out of the window.

duces large errors in the fits to the dispersion relations.

For this reason, the $\mathrm{HgCd}$ lamp has been used additionally to the $\mathrm{He}, \mathrm{Ar}$ and Ne lamps in the 2000 and 2001 observations. In Fig. 3.3 it is demonstrated how the number of usable emission features is increased this way.

The input catalogue which is used by the MIDAS routines for the calibration line search was thoroughly edited to ensure that it only contained emission features which are clearly detectable in the FORS spectra. This was done by comparing two calibration spectra (with very low starting and very high ending wavelengths, respectively) to the list of calibration lamp lines presented in the "FORS1+2 User Manual Issue 1.5" which was released by ESO in July 2000. A total of 45 lines were included in the final catalogue.

In the performance of the wavelength calibration, two of the most crucial parameters are the degrees of the polynomial fits to the dispersion

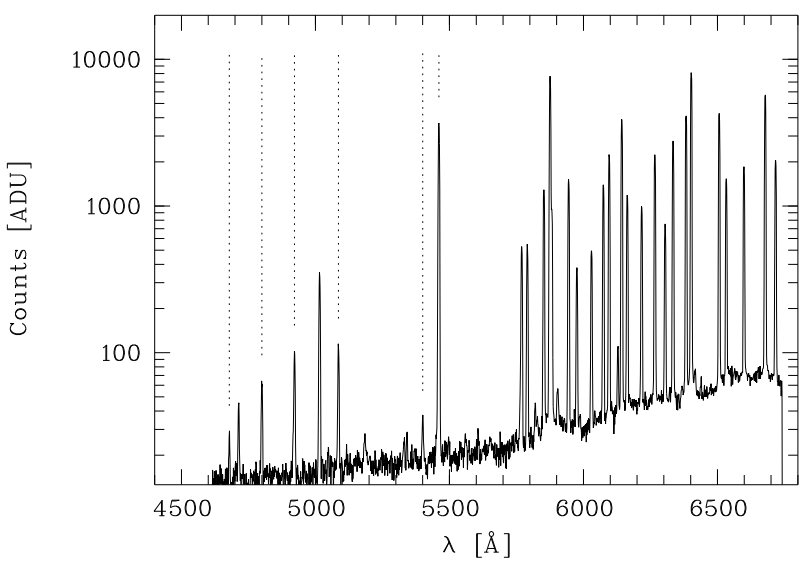

Figure 3.3: Wavelength calibration spectrum taken with FORS1. The dotted lines indicate the additional calibration lines below $\sim 5800 \AA$ by the $\mathrm{HgCd}$ lamp. The default combinaton of $\mathrm{He}, \mathrm{Ar}$ and Ne lamps only yields two lines in this wavelength range. Since the strengths of the displayed lines cover a broad range, the counts are given in logarithmic scale.

relation along the $\mathrm{X}$ axis and to the tilt of the emission lines along the $\mathrm{Y}$ axis, respectively. The fit degrees are held fixed for the entire spectrum (in the MIDAS algorithm RECTIFY/LONG), whereas the fit coefficients are assumed to be a function of position on the $\mathrm{Y}$ axis.

For the vast majority of the spectra, a third degree fit along the direction of dispersion yielded the best results. Various degrees of fits along the spatial axis were compared for the individual spectra. Here, first degree polynomials were used for roughly half of the spectra and second degree polynomials for the other half, respectively. In the case of two slits with extremely low starting wavelengths, the dispersion relations could not be fitted properly. These calibration problems were overcome by cutting off a portion of the spectra towards the low-wavelength end.

As a rule of thumb, one should aim for an r.m.s. of $0.1 \AA$ in the calibration process (e.g., Wagner 1992). The typical mean r.m.s. that was achieved with the FORS calibration spectra was 0.03-0.04 $\AA$ at a dispersion of $\sim 1.08 \AA /$ pixel. 


\subsection{Sky Subtraction}

The night sky subtraction was performed (using the MIDAS command SKYFIT/LONG) by fitting the sky emission column by column within windows on the $\mathrm{Y}$ axis that have to be defined by the user. An additional free parameter is the degree of the polynomial fit which is used to approximate the emission line intensity along the $\mathrm{Y}$ axis.

To quickly derive the appropriate individual windows on the $\mathrm{Y}$ axis for the night sky approximation, a small program was used. This routine automatically fitted and subtracted the night sky for every exposure of a MOS slit and derived the signal-to-noise ratios in the continua of onedimensional extractions of the resulting spectra. For objects which were roughly centered on a MOS slit, the maximum usable areas on both sides of the respective galaxy were used with a first or second degree fit. In the case of objects close to the edge of a slit, on the other hand, a zero degree fit was the best option. This was to be expected since for such a position, the night sky could only be determined properly on one side of the object. In effect, a higher degree polynomial fit resulted in large uncertainties of the coefficients and a decreased $\mathrm{S} / \mathrm{N}$ ratio.

A second modest cosmic ray removal was performed in the course of the night sky fitting. After subtraction of the fitted sky spectrum, the optical center of each galaxy continuum on the $\mathrm{Y}$ axis was determined by fitting a Gaussian profile. If necessary, the spectra were shifted along this axis by integer values (mostly one pixel, two pixels at maximum in a few cases) to achieve consistent optical centers in the exposures to within at least half a pixel, corresponding to 0.1 arcsec. A quadratic weighting was used in the final addition of the three slit exposures if their average DIMM seeing differed by more than $\sim 20$ percent.

One-dimensional extractions were derived by averging the rows within the FWHM of the fully reduced $2 \mathrm{D}$ spectra. Three examples of typical

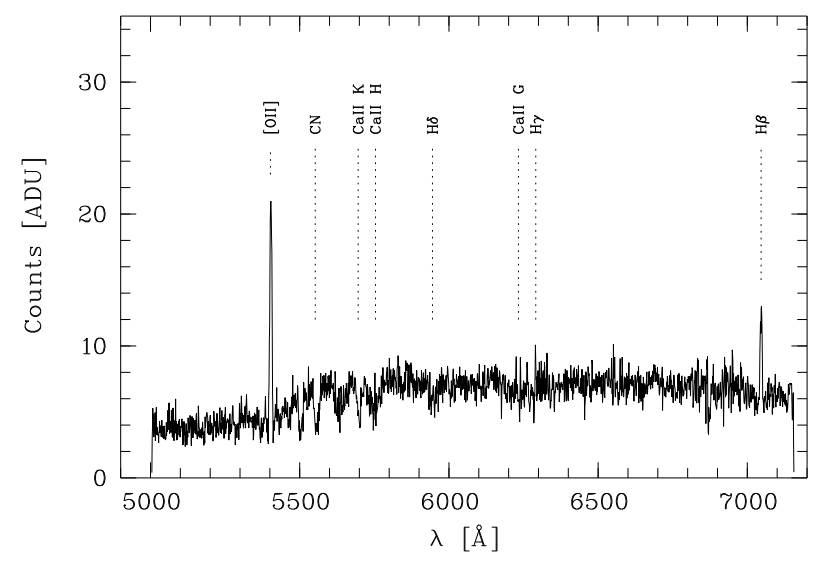

Figure 3.4: One-dimensional extraction of the fully reduced spectrum of the Sc spiral \#6125 with an apparent total brightness of $R=21.14^{m}$, shown in observer's frame.

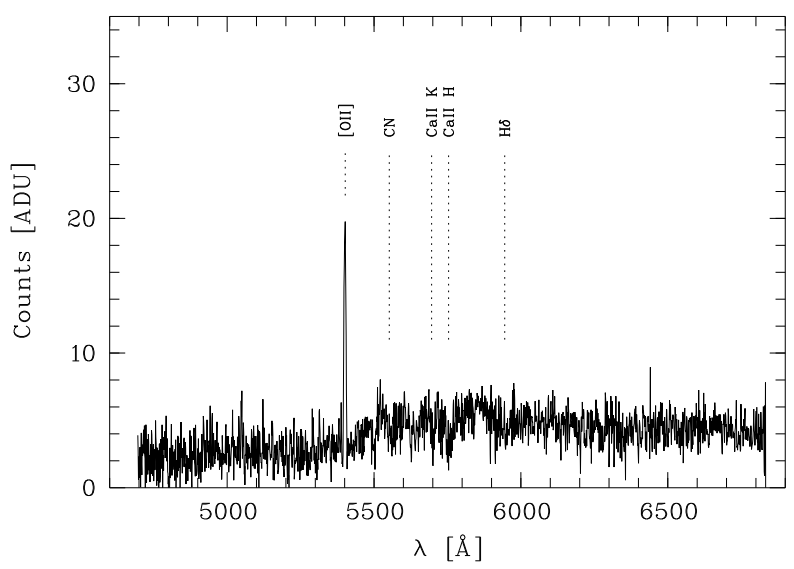

Figure 3.5: One-dimensional extraction of the fully reduced spectrum of the Sc spiral \#2572 with an apparent total brightness of $R=21.85^{m}$, shown in observer's frame.

FDF spiral SEDs classified as type Sc are shown in Figs. 3.4 to 3.6.

The galaxies have respective total apparent $R$ band brightnesses of $\sim 21^{m}, \sim 22^{m}$ and $\sim 23^{m}$. The numbers of the objects correspond to their IDs in the final photometric catalogue of FDF (Heidt et al. 2003) and in the data tables in the appendix.

Some characteristics of the spectra are also listed in Table 3.1. The columns give the ID, $R$-band brightness, redshift, mean seeing during the ob- 


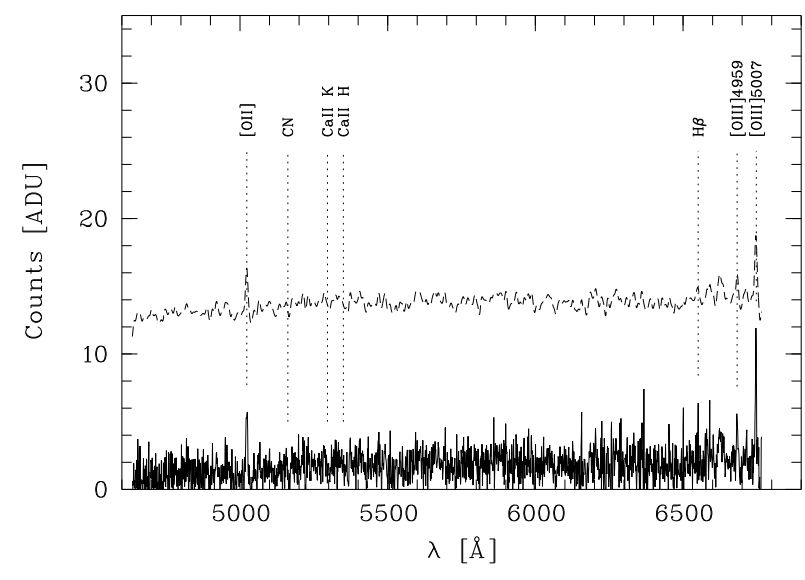

Figure 3.6: One-dimensional extraction of the fully reduced spectrum of the Sc spiral \#1834 with an apparent total brightness of $R=22.86^{\mathrm{m}}$, shown in observer's frame. The dashed overlayed spectrum has been smoothed with a Gaussian filter $\left(\sigma_{\lambda}=3.2 \AA\right)$ to improve the visibility of some absorption lines and is displayed with an offset of +12 ADU.

Table 3.1: Data of 3 FDF spirals

\begin{tabular}{cccccc}
\hline ID & $\begin{array}{c}R \\
{[\mathrm{mag}]}\end{array}$ & $z$ & $\begin{array}{c}\text { DIMM } \\
{[\operatorname{arcsec}]}\end{array}$ & $\begin{array}{c}\mathrm{S} / \mathrm{N} \\
\text { cont. }\end{array}$ & $\begin{array}{c}\mathrm{S} / \mathrm{N} \\
{[\mathrm{O} \text { II }]}\end{array}$ \\
\hline 6125 & 21.15 & 0.4495 & 0.43 & 10 & 30 \\
2572 & 21.90 & 0.4491 & 0.66 & 5 & 18 \\
1834 & 22.91 & 0.3475 & 0.82 & 1.5 & 6 \\
\hline
\end{tabular}

servation, $\mathrm{S} / \mathrm{N}$ per pixel in the continuum at $6050 \AA$ and in the [O II] doublet, respectively (the two latter values were derived from the $1 \mathrm{D}$ spectra). Note that the seeing conditions differed strongly between the three given objects. This selection was made to cover the range of data quality in the TF sample.

The data on the last spiral in table 3.1 illustrates that, even with the performance of a $10 \mathrm{~m}$-class telescope like the VLT, the $\mathrm{S} / \mathrm{N}$ bcame very low for objects with $R \approx 23^{m}$ at a seeing FWHM of roughly one arcsecond. These observing condi- tions represented the lowest data quality in the sample. Nevertheless, even object \#1834 eventually yielded a spatially resolved rotation curve extending out to three optical scale lengths.

\subsection{I-band Reference Frame}

One of the aims of the TF project was the derivation of the structural parameters (inclination, position angle, disk scale length) and the morphology of the FDF spirals. For this purpose, the original coadded images of the FDF were not the best possible choice. This was because the imaging had been performed under varying observing conditions. In the $I$-band, e.g., the seeing ranged from 0.46 arcsec to 0.89 arcsec FWHM. Nevertheless, the highest spatial resolution had been achieved with this filter. To simultaneously gain the highest possible resolution and a sufficient $\mathrm{S} / \mathrm{N}$ at the outer isophotes of the TF objects, the $10 I$-band frames with the best seeing from the FORS2 commissioning phase (November 1999) were combined.

The bias and dark subtraction, cosmics filtering and a first flat-fielding was performed by J. Heidt for these frames. It was found that the correction of large scale sensitivities using twilight flats yielded much better results than with dome flats. Since the latter had mean count rates of typically $\sim 10^{4} \mathrm{ADU}$, whereas the former fell into the regime $\sim 10^{2} \mathrm{ADU}$, it was likely that the large scale sensitivity of the FORS CCDs was not perfectly linear.

However, even when the twilight flats were applied, the reduced frames still featured background variations of up to 5 percent over the field-of-view. To overcome this problem, the sky background was fitted using the Source Extractor package (Bertin \& Arnouts 1996) with a mesh size of 256 pixel in each frame. The background fits were normalized to their median and the corresponding $I$-band images were divided by the fits. After a correction in this manner, the frames were flat to within a few 0.1 percent. 
Since the FDF imaging had been performed with a jitter pattern of 8 arcsec steps, the $10 I$-band frames were slightly offset from each other (120 pixel at maximum). Therefore, the images were shifted by integer values before they were skysubtracted and finally median-averaged.

The resulting reference frame with $3000 \mathrm{~s}$ total exposure time had a Point Spread Function of 0.49 arcsec FWHM. A straightforward calibration was perfomed via aperture photometry of five bright, non-saturated stars, of which the $I$ magnitudes already were determined during the calibration of the complete FDF imaging. The zero point of the reference frame was $32.05^{m}$. Using the Source Extractor with elliptical isophotes and the algorithm Mag_auto (see chapter 4.6.1), a $50 \%$ completeness limit of $\sim 25.1^{\mathrm{m}}$ was derived for the reference frame. The faintest spirals in the TF sample had a central $\mathrm{S} / \mathrm{N}$ between $10 \sigma$ and $15 \sigma$ of the sky noise level in this image. 


\section{Chapter 4}

\section{Spectrophotometric Analysis}

In many respects, the data quality of the FDF spiral sample is comparable with or even superior to that of other recent studies of distant spirals. As the spectroscopy has been performed solely with the VLT (a $10 \mathrm{~m}$-class telescope) at sub-arcsecond seeing, only three other ongoing studies offer a comparably powerful basis for the kinematic analysis. These are the projects described in Milvang-Jensen (2003) and Barden et al. (2003) which also made use of the VLT but comprised only 19 field spirals each, and a project within the Deep Extragalactic Evolutionary Probe (see Koo 2001) performed with the Keck 10 m-telescope on Mauna Kea (Hawaii). After first results from the latter study have been outlined in Vogt et al. $(1996,1997)$ on the basis of 17 galaxies, the complete set of $\sim 100$ spirals has been briefly discussed in Vogt $(2000,2001)$. Thanks to the very deep multi-band imaging, the basis for the derivation of the luminosities of the FDF spirals is more robust than that of any other distant field spiral sample - most of these were restricted to two filters, whereas for the FDF sample, four filters $(B, g, R$ and $I)$ are usable.

One issue that has to be considered, however, is the spatial resolution of the FDF imaging. The PSF of the reference $I$ frame which was used for the derivation of the structural parameters had a FWHM of 0.49 arcsec. In contrast to this, other studies of distant spirals mostly comprised HST imaging with a higher resolution. Images taken with the Wide Field and Planetary Cam- era 2 have $\sim 0.15 \operatorname{arcsec}$ FWHM, and the Advanced Camera for Surveys wich is operational since July 2002 even offers $\sim 0.09$ arcsec FWHM. Although the effect of instrumental resolution was accounted for in the surface brightness profile fits, as will be decribed in section 4.3, it was investigated whether the ground-based imaging could affect the results. This was achieved with a "VLT simulation" of the HDF-N which is the topic of section 4.4. Aided by a classification scheme that has been introduced by Abraham et al. (1996) especially for observations at low intrinsic spatial resolution, section 4.5 will focus on the question to what extent the spectral types correlate with the morphological types. The derivation of one of the basic parameters for the construction of the scaling relations, the absolute magnitudes, will be be outlined in section 4.6. Data tables containing the results presented in this chapter can be found in appendix A.

In the following two sections, details will be given on the redshift distribution and the spectrophotometric types of the FDF spirals.

\subsection{Redshift Distribution}

For the determination of the galaxies' redshifts and the spectral classification, an onedimensional extraction of each 2-D spectrum was utilized that has been constructed by averaging all rows within the FWHM of the profile along the spatial (Y) axis.

The search for emission and absorption lines 


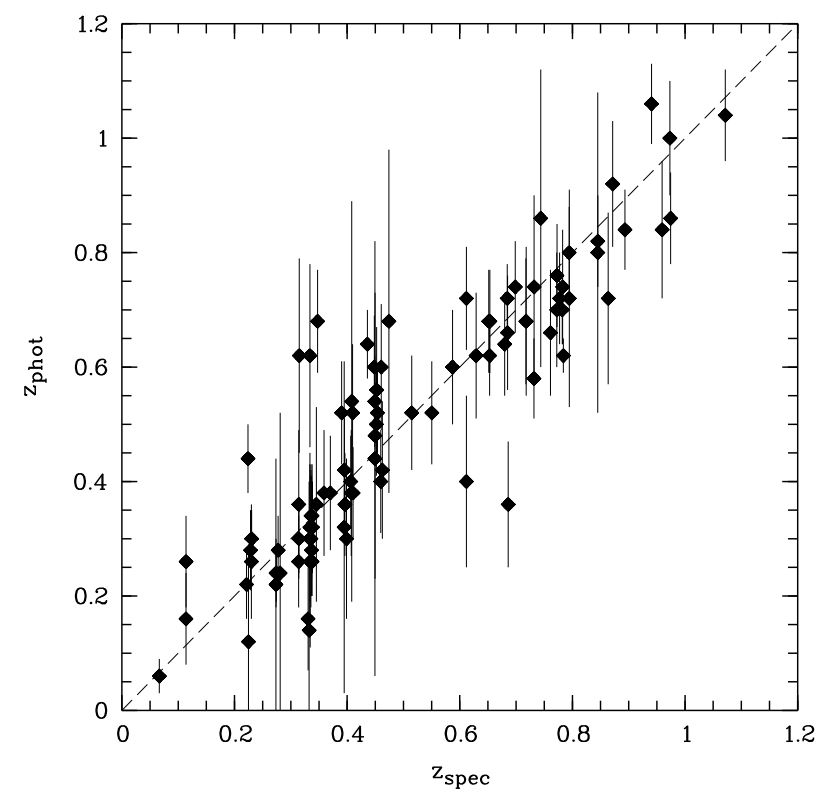

Figure 4.1: $\quad$ Spectroscopic vs. photometric redshifts for $98 \mathrm{FDF}$ spirals. 15 objects from preliminary versions of the photometric redshifts catalogue have been neglected due to unusually large errors. The two approaches are consistent within the errors for the vast majority of the galaxies.

then was performed in a semi-automatic manner. First redshift estimates were either taken from the photometric redshifts catalogue or byeye identifications of emission lines. A program then displayed the prominent lines of a typical spiral simultaneously with the spectrum to verify the estimated value of $z$. For the derivation of the exact redshift, Gaussian profiles were fitted to the identified lines to compute the central wavelength. The catalogue of lines which was used herein included the Balmer series, [O II] 3727, [O III] 4959, [O III] 5007, G, CaII H, CaII K, CN, $\mathrm{Mg}_{b}$ and FeIV.

In total, the redshifts of 113 spirals could be determined, including three cases of secondary objects which were covered by a MOS slit by chance. A comparison between the photometric and spectroscopic redshifts is presented in Fig. 4.1.

For 16 objects, including one secondary, it was not possible to derive a value of $z$. These galaxies populate the extreme faint end of the apparent brightness distribution with a median of $\langle R\rangle=22.9^{m}$. 13 of these objects are, based on the photometric redshifts catalogue, bona fide spirals. The three other objects are not included in any catalogue, nevertheless it is likely that they are spirals on the basis of their $B-R$ and $R-I$ colors.

Since only one of the 16 objects shows colors of a very late-type $\mathrm{SED}$, the $\mathrm{S} / \mathrm{N}$ of the remainders may be just too low to yield detectable emission lines. In the case of 8 targets, the redshifs might be slightly higher than estimated in the photometric redshifts catalogue, and the [O II] 3727 doublet thereby could be shifted out of the wavelength range of the R600 grism. For 5 other galaxies in the regime $0.2<z_{\text {phot }}<0.5$, only [O III] or $\mathrm{H} \beta$ emission is potentielly covered, which is weaker than $\mathrm{H} \alpha$ or [O II] for typical spirals. That is, the $\mathrm{S} / \mathrm{N}$ may be too low in these spectra to gain detectable emission lines.

Furthermore, the typical S/N of the spectra from the observations in 2001 was relatively low due to the faintness of the targets $\left(\langle R\rangle=22.8^{m}\right.$, see chapter 2.6) and slightly poorer seeing conditions than in 2000 . While only $\sim 29 \%$ of all FDF spirals were observed in 2001, 9 out of 16 spirals without a spectroscopic redshift originate from these setups.

Figure 4.2 shows the redshift distribution of all 113 late-type galaxies with determined redshifts and of 77 spirals which yielded rotation curves appropriate for the derivation of $V_{\max }$. The latter will be referred to as TF objects hereafter, see chapter 5.2.2 for a description of the constraints to the rotation curve quality. The median redshift of both data sets is $\langle z\rangle=0.45$. It is likely that the bimodal shape is a combination of the two following effects.

Firstly, the southwestern corner of the FDF most probably covers the outskirts of a galaxy cluster at $z=0.33$. Assuming a spread in redshift of $\Delta z=0.01$ (which corresponds to $\sim 1.5$ times the 


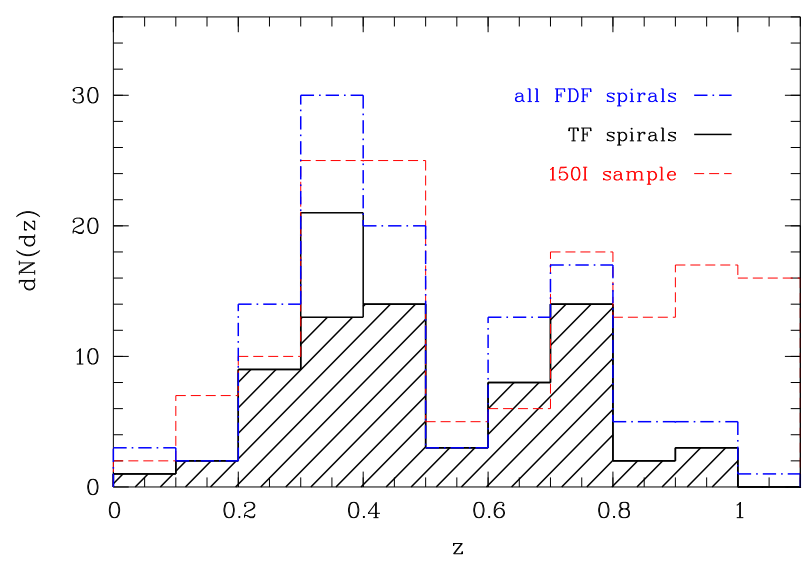

Figure 4.2: Redshift distributions of the full sample of 113 FDF spirals (dash-dotted line) and the 77 FDF spirals with rotation curves usable for the derivation of $V_{\max }$ (solid line). The shaded region delineates the distribution of the TF sample after further exclusion of 8 possible members of a cluster at $z=0.33$. As a comparison, the dashed line represents the lower resolution spectroscopy sample observed with grism 150I as part of the FDF high-z study (Noll et al. 2003). All data sets feature a redshift "gap" around $z \approx 0.6$.

typical velocity dispersion of a rich galaxy cluster, $\sigma_{\mathrm{v}} \approx 1000 \mathrm{~km} / \mathrm{s}$ ), it is found that a maximum of 13 spirals with derived redshifts and of $8 \mathrm{TF}$ objects could be members of this cluster. The small redshift "bump" at $z \approx 0.3$ can be attributed to these objects.

The spatial distribution of all potential cluster members is shown in Fig. 4.3. It is suggestive that the cluster has an elongated shape. If the center indeed is positioned to the southwestern corner of the FDF, the distribution of the galaxies will be a striking example of the morphologydensity relation (Dressler 1980) with the earlytype galaxies mainly populating the dense, inner region and the late-type galaxies at larger clustercentric radii. For the major parts of the further analysis, the spirals which could be cluster members will not be treated separately (however, cf. chapter 6.4.3).

A second reason for the bimodal shape of the redshift distribution is the relatively low number

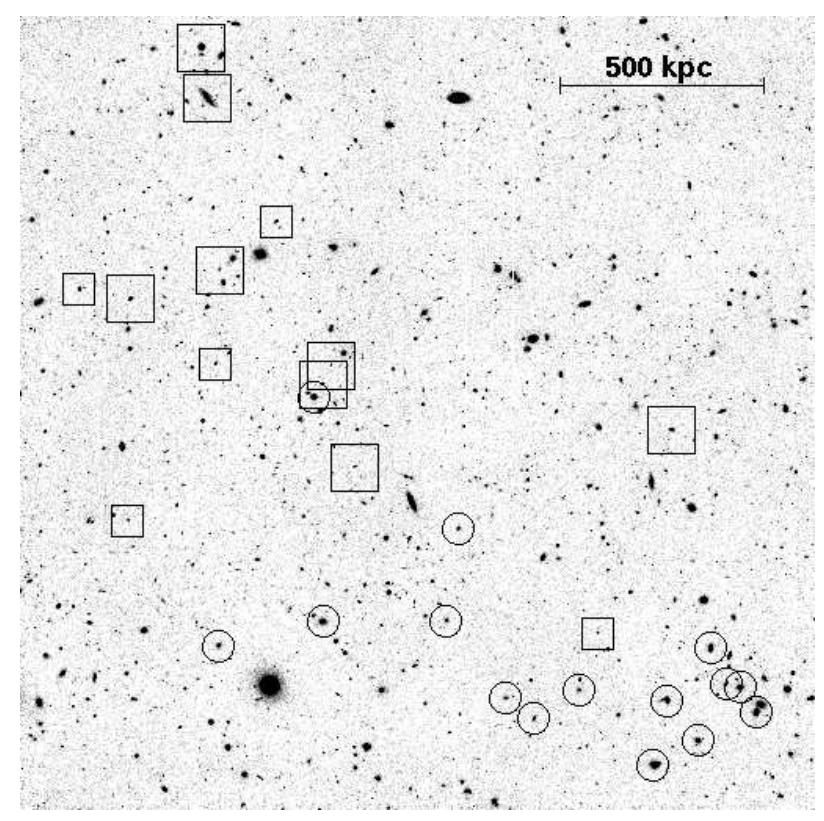

Figure 4.3: Possible members of a cluster in the southwestern (lower right) corner of the FDF. 15 early-type galaxies are denoted by circles, 13 latetype galaxies by squares. The eight potential cluster spirals which could be used for the $V_{\max }$ derivation are depicted by large squares. The bar corresponds to $500 \mathrm{kpc}$ in projection at the clusters redshift of $z=0.33$.

of galaxies at $z \approx 0.6$. As a comparison, the distribution of 144 galaxies at $z<1.1$ from the FDF high $-z$ campaign (Mehlert et al. 2002, Noll et al. 2003 ) is additionally shown in Fig 4.2. Note that this sub-sample excludes 39 spirals which were also observed within the TF project. The high$z$ spectra were taken with the same instrument configuration as the TF data, except that the low-resolution grism 150I was used which covers a much broader wavelength range of $\sim 6000 \AA$ in observer's frame. The rather high fraction of galaxies with $z \leq 1$ which did not enter the TF survey can be attributed to the much fainter brightness limit of the high- $z$ study $\left(I \leq 24.5^{m}\right)$, the inclusion of elliptical galaxies and the lack of constraints to the inclinations. It is clear from Fig. 4.2 that both samples feature the same redshift "gap". 
Therefore, it is likely that the volume probed with the FDF contains less galaxies at $z \approx 0.6$ than the neighbouring redshift bins and that the redshift distribution of the TF spirals is not a selection or observational effect.

\subsection{Spectral Classification}

The FDF spirals were classified in terms of their SEDs. A rough morphological classification into normal spirals and irregulars has also been performed and is outlined in section 4.5, but the attempt to divide the sample into sub-types was purely based on the spectra.

To conduct such a classification, three criteria were used. The SED parameter model from the photometric redshifts catalogue, which is related to the star formation e-folding time of the bestfitting templates, ranged from a value of 3 for an early-type spiral to 12 for a very late-type spiral. These values were transformed to the de Vaucouleurs scheme $T$ which ranges from $T=1$ for an Sa spiral to $T=10$ for the type Im. This was useful especially for spectra with very few identifiable lines and/or low $\mathrm{S} / \mathrm{N}$, but had the disadvantage that dust reddening might have caused a classification biased towards early types for highly inclined spirals.

Because of this, a comparison to the SED templates in the Kennicutt catalogue (Kennicutt 1992a) has also been performed. Since the $\mathrm{H} \alpha$ line was covered by the wavelength range of the grism only in the case of four TF spirals, this byeye comparison mainly focussed on [O II] 3727 , $\mathrm{H} \beta$ and [O III]. For spectra without detectable [O III] emission, the respective spirals were assumed to be early-type, and the strength of [O II] or $\mathrm{H} \beta$ emission could be used to distinguish between the types $\mathrm{Sa}$ and $\mathrm{Sb}$. For later types, the relative line strength of $\mathrm{H} \beta$ and [O III] 5007 was used to sub-divide into Sc and Sdm. When the [O III] 4959 emission was stronger than $\mathrm{H} \beta$, the spectrum was classified as Im.

As a third indicator, the [O II $] 3727$ equivalent widths (EWs) were measured. This line doublet was visible in the spectra of 69 spirals in total and $49 \mathrm{TF}$ objects in particular. First, double Gaussian profiles were fitted to each doublet (with the MIDAS algorithm DEBLEND/LINE) yielding the integrated flux of the doublet. The continuum flux was derived on both sides of the [O II] emission. Then, the equivalent width $W$ in observer's frame was calculated via

$$
W=\frac{2}{F_{l}^{c}+F_{r}^{c}} \int_{\lambda_{1}}^{\lambda_{2}}\left(F_{\lambda}-F_{\lambda}^{c}\right) d \lambda .
$$

Here, $\lambda_{1}$ and $\lambda_{2}$ define the start and ending wavelength of the central window for the derivation of the integrated line flux (typically 20-30 $\AA$ ), $F_{l}^{c}$ and $F_{r}^{c}$ are the continuum fluxes $\sim 5 \AA$ to the left and $\sim 5 \AA$ to the right of the wavelength window. The continuum flux $F_{\lambda}^{c}$ in Eq. 4.1 was approximated linearly from the two sampling points of the continuum, i.e.

$$
F_{\lambda}^{c}=F_{l}^{c}+\left(F_{r}^{c}-F_{l}^{c}\right) \frac{\lambda-\lambda_{l}}{\lambda_{r}-\lambda_{l}} .
$$

Finally, the rest-frame equivalent width $W_{0}$ was computed via $W_{0}=W /(1+z)$ and correlated to a spiral sub-type by comparison to the typedependent EWs of local spirals given by Kennicutt (1992b).

Only for a fraction of the FDF spirals, all three criteria could be applied. At higher redshifts, e.g., [O II] was the only visible emission line, therefore a by-eye comparison to the Kennicutt catalogue was not appropriate. On the other hand, the [O II] EWs offered a robust, quantitative criterium in these cases.

The resulting classifications by the respective criteria were combined, with a preference of the second and third criterium since they were based on a much higher spectral resolution than the photometric redshifts catalogues which were restricted to the broad imaging. Five sub-types were considered in the final SED classification scheme: $T \in\{1,3,5,8,10\}$ corresponding to Sa, $\mathrm{Sb}, \mathrm{Sc}, \mathrm{Sdm}$ and $\mathrm{Im}$. 
The TF sample of 77 galaxies comprised 14 spirals of type $T \leq 3$ in the redshift range $0.11 \leq z \leq 0.89$ with a median of $\langle z\rangle=0.42$, 43 galaxies with $T=5$ covering $0.09 \leq z \leq 0.97$ with $\langle z\rangle=0.45$ and 20 objects with $T \geq 8$ in the regime $0.23 \leq z \leq 0.97$ with $\langle z\rangle=0.51$. This high degree of type-independence in the redshift distribution was highly valuable for the analysis of the TFR.

\subsection{Surface Brightness Profile Fitting}

As has been outlined previously, the use of a rather simple photometry package like the Source Extractor was not appropriate for an accurate derivation of the structural parameters, i.e. the inclinations, the position angles and the scale lengths of the FDF spirals. The reason for this is that the Point Spread Function (PSF) for ground-based images in particular - heavily affects the isophotal shape of a galaxy. Various programs have been developed in the recent years for the more "sophisticated" approach of surface brightness profile fitting, of which the majority offer the consideration of the observed PSF. One example is the oftenly used package Galaxy Image Two-Dimensional (GIM2D, see Simard et al. 2002), which operates within the environment of the IRAF (Image Reduction and Analysis Facility) software maintained by the National Optical Astronomy Observatory Observatories in Tucson, Arizona.

However, for two reasons it was decided to write a special program for the profile fitting. Firstly, this had the advantage that every detail of the applied algorithms was known and no "black boxes" were involved in the analysis. Moreover, publicly available packages like GIM2D have been applied almost solely to HST images and it is not clear whether the performance would be comparably high on ground-based images. Indeed, one of the key questions in terms of the structural parameters of the FDF spirals was to what extent the VLT imaging could compete with space-based data. This topic will be discussed in the next section.

The program for the performance of the surface brightness profile fits (called diskmodel) used four free parameters. These were:

- inclination $i$

- position angle $\theta$

- apparent scale length $r_{\mathrm{d}}$

- central flux $I_{c}$

The instrumental PSF was an essential input parameter of the algorithm and could either be supplied as a two-dimensional image of a stellar FDF object or as a scalar to define the FWHM in arcseconds. It was tested with a range of very steep synthetic profiles (FWHM $\ll 0.1$ arcsec) that this algorithm yielded the appropriate PSF value to within $<1 \%$.

In both alternatives to define the spatial resolution, the variation of the PSF as a function of CCD position, i.e., as a function of the position of the respective galaxy within the $I$ reference frame, was taken into account. This was achieved with six unsaturated stars in the range $18^{m}<I<20^{m}$ covering almost the complete FOV of the FDF. For each galaxy, its nearest neighbor among these reference stars was used to define the input PSF.

Five additional input parameters were the initial stepsizes of $i, \theta, r_{\mathrm{d}}$ and $I_{c}$, and the scale of the image which was held fixed to $0.2 \mathrm{arcsec} / \mathrm{pixel}$ for all FDF spirals. The observed galaxy profiles were read in as quadratic thumbnails. Depending on the apparent size of the objects, these thumbnails covered an area between $11 \times 11$ and $51 \times 51$ pixels. Upper and lower limits on the free parameters could also be defined.

At each point in the parameter space spanned by $i, \theta, r_{\mathrm{d}}$ and $I_{c}$, diskmodel firstly generated a synthetic two-dimensional exponential disk profile

$$
I(x, y)_{\mathrm{syn}}=I_{c} e^{-\left(r / r_{\mathrm{d}}\right)}
$$




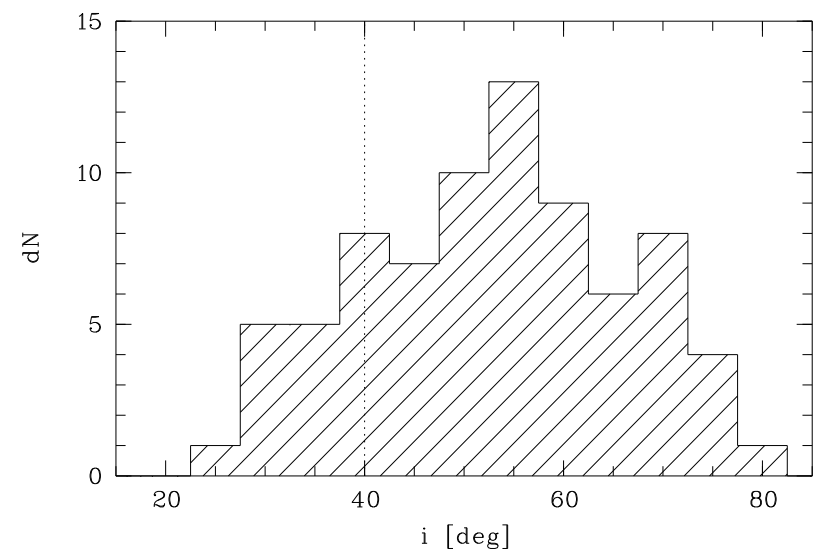

Figure 4.4: $\quad$ Distribution of the inclinations of 77 FDF spirals which could be used for the derivation of the maximum rotation velocity, the $\mathrm{TF}$ sample. The necessity to partly exceed the initial inclination limit during in the MOS target selection process (indicated by the dotted line) yielded $13 \mathrm{TF}$ galaxies with $i<40^{\circ}$.

with the transformation equations

$$
\begin{array}{r}
r^{2}=x_{1}^{2}+y_{1}^{2} \\
x_{1}=x \cos \theta+y \sin \theta \\
y_{1}=(x \sin \theta-y \cos \theta) / \sin i
\end{array}
$$

The synthetic profile then was convolved with the specified PSF yielding a profile $I(x, y)_{\text {con }}$. Finally, $\chi^{2}$ was computed via

$$
\chi^{2}=\sum_{x} \sum_{y} \frac{\left(I(x, y)_{\mathrm{obs}}-I(x, y)_{\mathrm{con}}\right)^{2}}{\sigma_{I}(x, y)^{2}}
$$

with an error $\sigma_{I}(x, y)$ which was assumed to be the standard deviation of the flux in a box of $3 \times 3$ pixels centered on $(\mathrm{x}, \mathrm{y})$.

In a first iteration, the program covered the complete parameter space in order to avoid convergence to a local minimum of $\chi^{2}$. After this, the algorithm started a new iteration with decreased stepsizes and narrower ranges for $i, \theta, r_{\mathrm{d}}$ and $I_{c}$, centered on the minimum $\chi^{2}$ from the first iteration. To limit the computation time, the number of iterations was mostly set to two or three.

The best-fitting values for the inclinations of the $\mathrm{TF}$ spirals ranged from $25^{\circ}$ to $80^{\circ}$ with a median

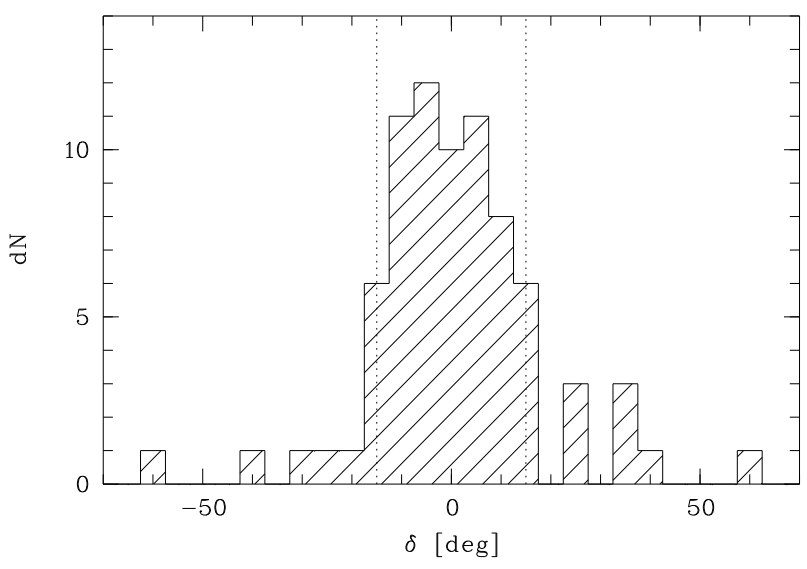

Figure 4.5: Distribution of the misalignment angles $\delta$ between the apparent major axis and the MOS slit for the $77 \mathrm{TF}$ spirals. The two dotted lines indicate the initial target selection criterium $|\delta| \leq 15^{\circ}$.

value of $\langle i\rangle=53^{\circ}$. Figure 4.4 shows the distribution of the inclinations. Based on the measured position angle $\theta_{\mathrm{obj}}$, the effective misalignment angle $\delta$ was computed from the position angle of the slit, $\theta_{\text {slit }}$, via

$$
\delta=\theta_{\text {obj }}-\theta_{\text {slit }} .
$$

$\delta$ therefore denotes the position angle of a galaxy with respect to the spatial axis of the corresponding MOS mask. The distribution of these misalignment angles is given in Fig. 4.5. Note that not only the absolute values but also the signs of $\delta$ were taken into account in the $V_{\max }$ derivation process (next chapter).

One shortcoming of the ground-based imaging was that a potential bulge component, i.e., an additional de Vaucouleurs profile, could not be resolved except for a few early-type spirals at low redshifts. Therefore, only the profiles of the disks were fitted. This decision was made because of the relatively low speed of the program - the computation time for typical FDF spirals ranged between 10 and 60 minutes, depending on the initial cuts on the parameter space. Thus, the number of free parameters was limited to four. Whether the lack of a two-component fit could introduce a systematic error in the structural pa- 


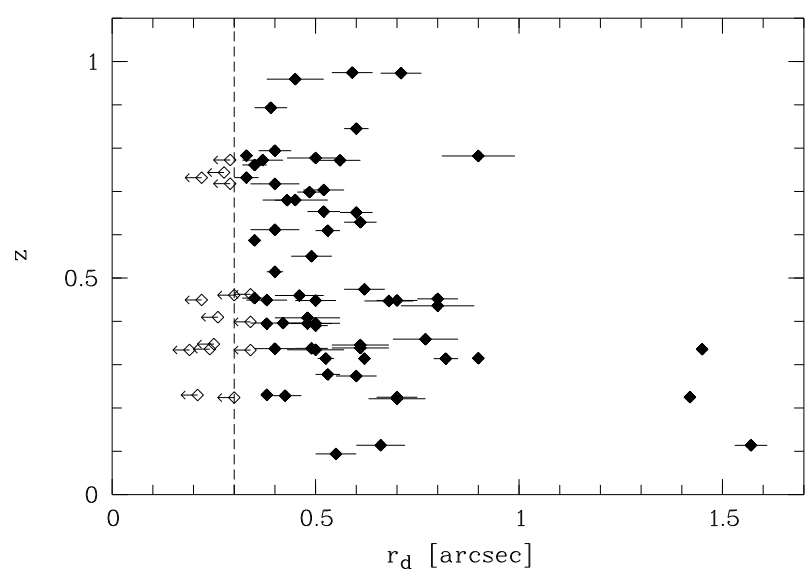

Figure 4.6: Apparent scale lengths of the TF spirals plotted against redshift. 15 Objects which have scale lengths below 0.3 arcsec within the errors could be unresolved (open symbols) and the best-fitting values of $r_{\mathrm{d}}$ may give only upper limits for these galaxies.

rameters was tested with the SED sub-types. If a significant fraction of the early-type FDF spirals had a prominent visible bulge, then the ellipticities of their isophotes could be too low for their disk inclinations. For spirals with late-type SEDs, on the other hand, it is very unlikely that they possess a significant bulge component. In effect, the best-fitting inclinations would be biased towards lower values for earlier types. However, the median inclinations of the $\mathrm{TF}$ spirals are $\langle i\rangle=51^{\circ}$ for $T \leq 3,\langle i\rangle=53^{\circ}$ for $T=5$ and $\langle i\rangle=51^{\circ}$ for $T=8$, respectively. It is therefore unlikely that the inclinations are affected by a bias of type-dependence. Further tests of the reliability of the one-component fits will be presented in the two following sections 4.4 and 4.5. The best-fitting scale lengths of the TF spirals ranged from 0.19 arcsec to 1.5 arcsec with a median of 0.45 arcsec. The distributions of the apparent and absolute values are presented vs. the redshifts in Figs. 4.6 and 4.7, respectively. Under the assumption that all spirals with an FWHM equal to or greater than the FWHM of the reference frame's PSF are resolved, it is found that the scale lengths of $15 \mathrm{TF}$ objects may be only upper limits. It can be deduced from Fig. 4.6

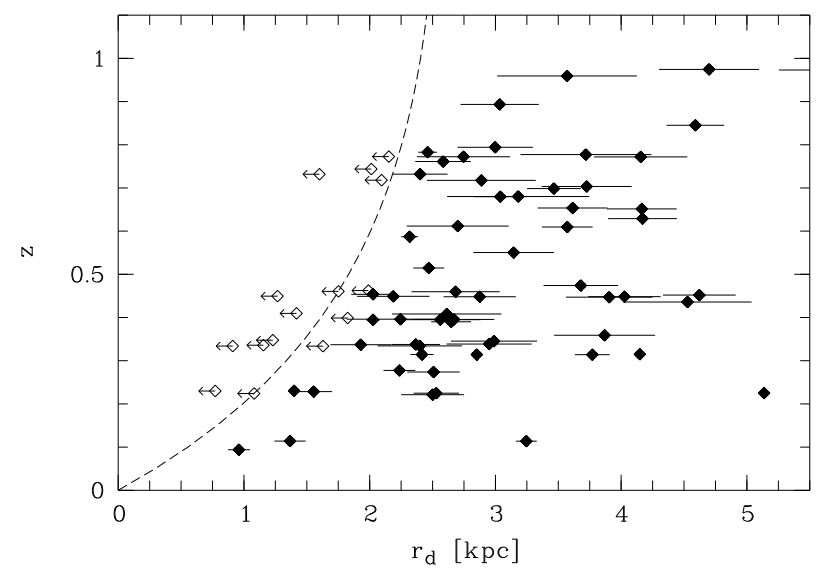

Figure 4.7: Absolute scale lengths $r_{\mathrm{d}}$ of the TF spirals plotted against redshift. As in Fig. 4.6, the dashed line denotes the probable lower limit on $r_{\mathrm{d}}$ for the spirals to be resolved. At $z=0.5$, e.g., this limit of 0.3 arcsec corresponds to $\sim 1.8 \mathrm{kpc}$.

that the fraction of potentially unresolved spirals does not - as one might expect - increase with redshift.

On the other hand, one could speculate on a slight increase of the absolute scale lengths with increasing redshift in Fig. 4.7. However, this apparent trend is just an effect caused by the target selection constraint on the $R$-band brightness. Towards higher redshifts, only luminous and therefore large spirals fulfill the criterion $R \leq 23^{m}$ (the correlation between luminosity and scale length is the well-known Freeman relation, see the introduction). In fact, one must consider the evolution of the relation between $V_{\max }$ and $r_{\mathrm{d}}$ to analyse a potential trend with look-back time. For a given $V_{\max }$, the TF sample shows evidence for a slight decrease of the disk sizes with redshift (chapter 6.3) as is to be expected in a scenario of hierarchical structure formation.

Errors on the fitted parameters were computed with diskmodel via the errors of the minimum $\chi^{2}$ which were defined as

$$
\sigma_{\chi^{2}}=\sqrt{2 \nu}
$$

where $\nu$ denoted the degrees of freedom, i.e., the 
number of pixels in the thumbnail image of an object.

As an example of how the errors have been calculated, the derivation of the error on the bestfitting inclination $i_{\text {fit }}$ will be explained in the following. With the other parameters $-\theta, r_{\mathrm{d}}$ and $I_{c}$ in this case - held fixed at their $\chi_{\min }^{2}$ values, the inclination corresponding to

$$
\chi_{\sigma}^{2}=\chi_{\min }^{2}+\sigma_{\chi^{2}}
$$

was derived. For the vast majority of the galaxies, one lower limit $i_{1}<i_{\text {fit }}$ and one upper limit $i_{2}>i_{\text {fit }}$ fulfilled this condition. These inclinations then were used to estimate a lower $1 \sigma$ limit

$$
\sigma_{i}^{-}=i_{\mathrm{fit}}\left(\chi_{\min }^{2}\right)-i_{1}\left(\chi_{\sigma}^{2}\right)
$$

and an upper $1 \sigma$ limit

$$
\sigma_{i}^{+}=i_{2}\left(\chi_{\sigma}^{2}\right)-i_{\text {fit }}\left(\chi_{\min }^{2}\right) .
$$

In most cases, the errors were very symmetric, i.e. $\chi^{2}(i)$ was well reproduced by a polynomial of even degree. For the sake of simplicity, the inclination error $\sigma_{i}$ was therefore defined as

$$
\sigma_{i}=\left(\sigma_{i}^{-}+\sigma_{i}^{+}\right) / 2 .
$$

Reduced values of $\chi_{\min }^{2}$ were computed via

$$
\chi_{\text {red }}^{2}=\chi_{\min }^{2} / \nu
$$

With a median of $\left\langle\chi_{\text {red }}^{2}\right\rangle=0.86$, the reduced $\chi^{2}$ values indicate satisfying fits for the majority of the spirals - according to the rule-ofthumb for this fitting method, that the regime $\chi_{\text {red }}^{2}<1$ qualifies for a "good" fit (e.g., Press et al. 1992). However, this may be partly introduced by the limitations on the spatial resolution since slight asymmetries or peculiarities may be smoothed by the instrumental PSF. This topic will be adressed also in the next two sections.

\subsection{An HDF Simulation}

Although the first tests showed that the method of fitting the FDF spiral profiles with a single disk component was unlikely to have introduced a systematic error, it was desirable to compare the performance of diskmodel against an established and publicly available fit program. Furthermore, the reliability of the ground-based data in recovering the structural parameters in general and the inclinations in particular remained an open question.

An approach to explore both latter issues simultaneously is to compare the results yielded by diskmodel on the basis of VLT imaging to the results of a well-tested program based on HST data of the same sky area. This was achieved in the following way.

As a reference, the results from Marleau \& Simard (1998, MS98 hereafter) were used. The authors derived the structural parameters of 522 galaxies with apparent magnitudes $I \leq 26^{m}$ in the HDF-N using the GIM2D package (Simard et al. 2002). The data table of the complete sample has been made public and was retrieved via anonymous ftp (cdsarc.u-strasbg.fr/pub/cats/J/ApJ/507/585/). All drizzled images from the HDF-N which were used in MS98 were also publicly available and retrieved from http://stdatu.stsci.edu/hst/hdf/v2/drizzled/. The field comprised one frame for each of the four CCDs of the Wide Field and Planetary Camera 2, but since MS98 only used the three WF camera images, these will be referred to the following.

According to the information given on the homepage of the Space Telescope and Science Institute, the three drizzled F $814 \mathrm{~W}$ frames featured a total integration time of $\sim 34 \mathrm{hrs}$ and a $10 \sigma$ limiting magnitude of $27.6^{m}$ for an aperture of $0.2 \operatorname{arcsec}^{2}$. The scale was $0.04 \mathrm{arcsec} / \mathrm{pix}$ and the instrumental PSF which was estimated with five stellar objects had a FWHM $\approx 0.16$ arcsec.

By adopting the color-insensitve transformation to Cousins $I_{C}$ given by Fukugita et al. (1995), $\left(\mathrm{F} 814 \mathrm{~W}-I_{C}\right)=0.04^{m}$, and the transformation from FORS $I$ to Cousins $I_{C}$ derived by synthetic photometry as described in section 4.6.4, 


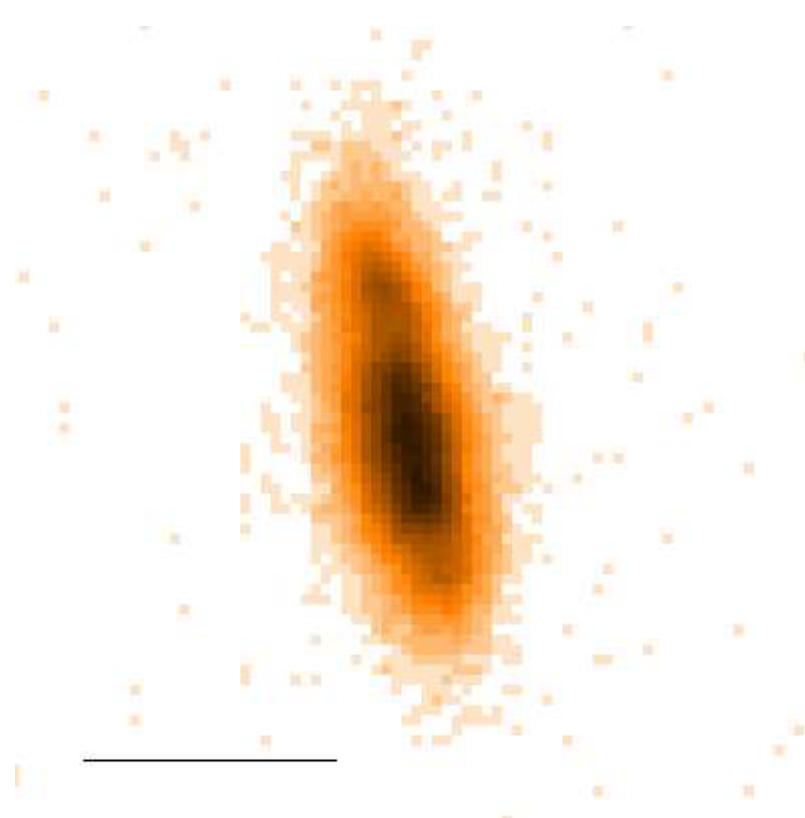

Figure 4.8: A spiral from the original drizzled HDF$\mathrm{N}$ images with $I_{814}=22.71^{m}$. According to the GIM2D values given by Marleau \& Simard (1998), the galaxy has an inclination $i=76^{\circ}$, a scale length $r_{\mathrm{d}}=0.32$ arcsec and a position angle $\theta=-78^{\circ}$. The bar corresponds to one arcsecond.

$\left(I_{\text {fors }}-I_{C}\right)=0.04^{m}$, the offset between $I_{\text {fors }}$ and $\mathrm{F} 814 \mathrm{~W}$ magnitudes was calculated to be $\left(\mathrm{F} 814 \mathrm{~W}-I_{\text {fors }}\right)<0.01^{m}$. Note that all three transformations are independent of the SED type to within $0.01^{m}$.

To be able to quantify the influence of the degraded spatial resolution of the ground-based frames on the profile fitting, an imaging of the HDF-N with the VLT was simulated. This was done by a transformation of the HST frames in two stages. First, all images were smoothed to a PSF of 0.49 arcsec to match the quality of the FDF $I$ reference frame. In a second step, the convolved images were rebinned to the FORS scale of $0.2 \mathrm{arcsec} / \mathrm{pix}$, i.e., the total number of resolution elements was reduced by a factor of 25. Hence, the resulting HDF-N frames had the same spatial resolution as the $I$ reference frame. The appearance of a spiral in the fully resolved HDF-N and in the VLT simulation image are

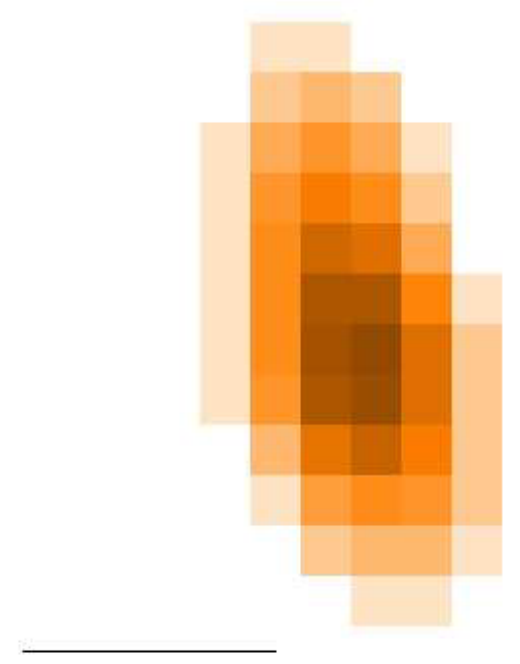

Figure 4.9: Same galaxy as in Fig. 4.8, but from the simulated VLT image of the HDF-N. The convolution and rebinning had a striking effect on the spatial resolution. Nevertheless, according to the fit with diskmodel on this image, the respective structural parameters are $i=77^{\circ}, r_{\mathrm{d}}=0.29$ arcsec and $\theta=-80^{\circ}$, which is in very good agreement with the original GIM2D output. The bar again corresponds to one arcsecond.

compared in Figs. 4.8 and 4.9.

An estimation of the limiting magnitude of the VLT simulation was performed with the Source Extractor on the basis of total apparent magnitudes measured with the Mag_auto algorithm. This yielded a 50\% completeness limit of $I \approx 26.2^{m}$. In comparison, the FDF $I$ reference frame - which comprised a total integration time $\sim 40$ times smaller - had a $50 \%$ completeness limit of $I \approx 25.1^{\mathrm{m}}$. The fact that the convolved HDF-N frames were substantially "deeper" than the FDF reference image had to be taken into account in the object selection for the test of the diskmodel performance, to ensure that the accuracy of the structural parameters of the FDF spirals could be tested indirectly.

This was achieved by the consideration of HDF 


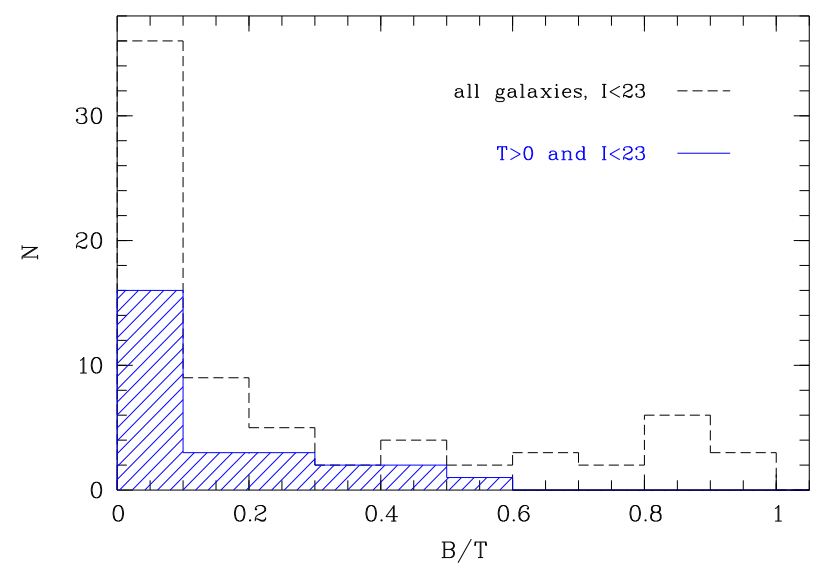

Figure 4.10: Bulge-to-total ratios of galaxies brighter than $I=23^{m}$ in the HDF-N as derived with GIM2D by Marleau \& Simard (1998). The shaded histogram gives the distribution of bona fide spirals according to the morphological classification by van den Bergh et al. (1996).

galaxies down to $I=23^{m}$. On the basis of the original GIM2D results presented in MS98, a sub-sample was extracted from their data table using the following constraints.

To exclude potential stellar objects, a limit of $r_{\mathrm{d}}>0.1$ arcsec was applied. Face-on disks which of course would not have been representative for the inclinations of the FDF spirals were also rejected with a criterion of $i>30^{\circ}$.

Furthermore, objects with bulge-to-total ratios of $B / T>0.6$ were rejected. This limit was derived from the distribution of all $I<23^{m}$ galaxies which were included in the GIM2D analysis as presented in Fig. 4.10. Only $\sim 24 \%$ of the galaxies with $I<23^{m}$ had $B / T>0.6$. In particular, the latter limit was not exceeded by any of the galaxies which were visually classified as spirals (i.e., objects with $T>0$ ) by van den Bergh et al. (1996, hereafter vdB96). The latter study did not cover all objects analysed in MS98, nevertheless a constraint on the bulge-to-total ratio could be determined to exclude early-type galaxies from the GIM2D sample.

After a few blended objects were also rejected upon visual inspection, a data set of 39 galax- ies (which will hereafter be referred to as the "MS sample") remained with a median apparent magnitude of $\langle I\rangle \approx 22.3^{\mathrm{m}}$. This was $\sim 1.1 \mathrm{mag}$ fainter than the median of the $77 \mathrm{TF}$ spirals, thus the difference in the integration times of the convolved HDF-N frames and the FDF reference image was exactly counter-balanced.

Besides the spatial resolution, an alternate way to quantify the image quality is the number of resolution elements, i.e., the number of pixels which can be used to fit the profile of an object. For this, one has to define a lower limit. With the Source Extractor, the numbers of pixels per object which are $>2 \sigma$ above the sky noise level were derived. At the faint ends of the respective brightness distributions, this number corresponded to $N_{\text {pix }} \approx 100$ per galaxy for the MS sample as well as for the FDF TF sample. This strengthened the conclusion that, with respect to these two data sets, the simulated VLT frames of the HDF-N and the $I$ reference frame had the same quality.

All 39 galaxies in the MS sample were analysed with diskmodel. For this, thumbnails of the respective objects were extracted from the corresponding VLT simulation of one of the three HDF-N images. Most of the thumbnails covered $21 \times 21$ pixels. Some very faint galaxies were fitted using $11 \times 11$ boxes, whereas one spiral with $I=20.0^{m}$ was extracted with an enlarged window of $31 \times 31$ pixels.

Three examples of profile fits are shown in Fig. 4.11. Observed and fitted fluxes were transformed to magnitudes per $\operatorname{arcsec}^{2}$ with the equation

$$
\mu(x, y)=-2.5 \log I(x, y)-3.495+29.685
$$

Here, $I$ is the flux in ADU at the position $(x, y)$, the constant $2.5 \cdot \log 25=3.495$ accounts for the spatial scale of $0.2 \mathrm{arcsec} / \mathrm{pixel}$ and the last term is the zero point of the rebinned and convolved HDF-N frames. Since the redshifts were unknown, the cosmological dimming factor of $(1+z)^{4}$ has not been applied. According to 

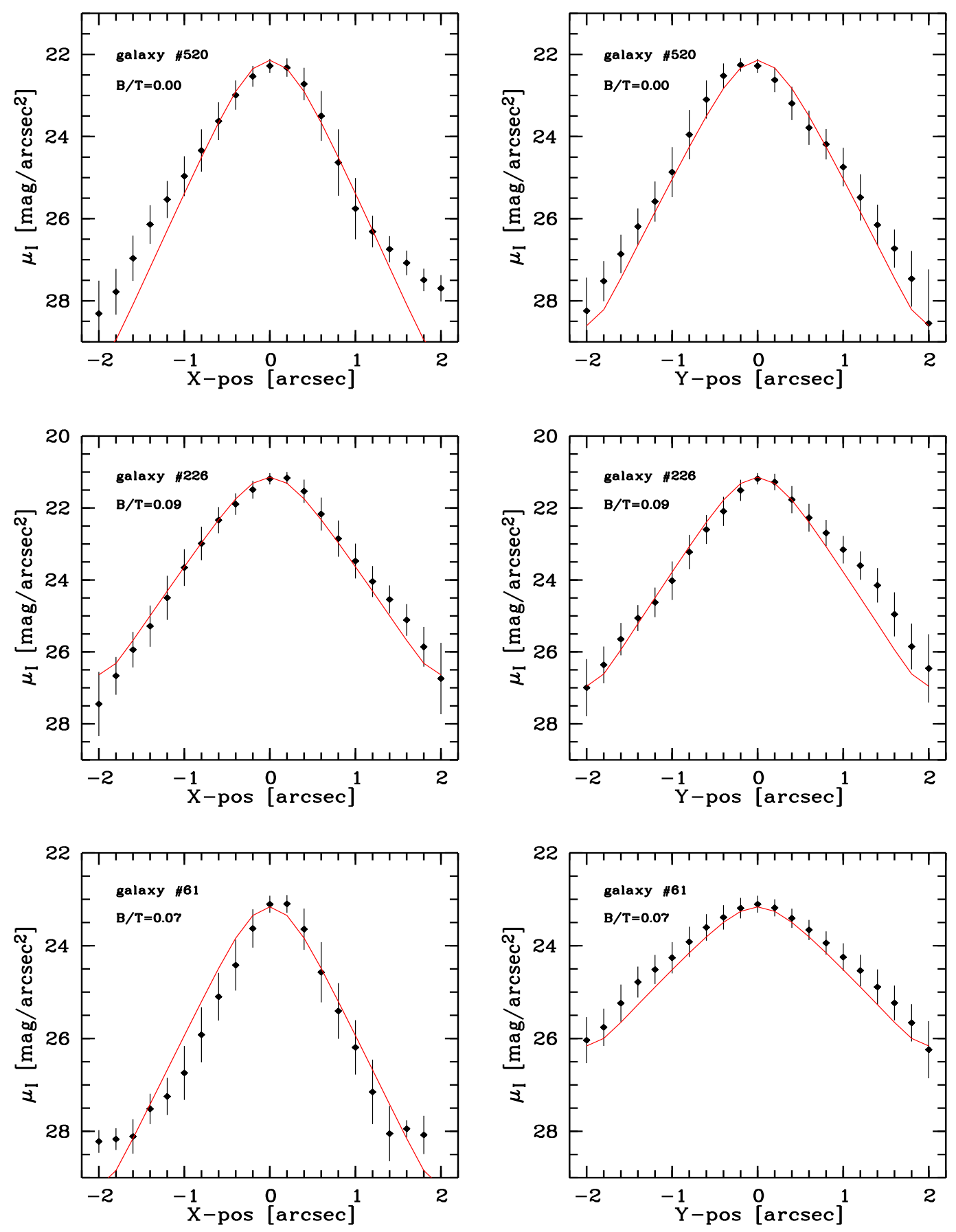

Figure 4.11: Surface brightness profiles of three galaxies from the simulated VLT imaging of the HDF-N. Observed profiles are denoted by the individual data points, the fits derived with diskmodel are shown as solid lines. On the left hand side, one-dimensional extractions along the $\mathrm{X}$-axis are shown, whereas the profiles on the right hand side were extracted along the Y-axis. Error bars correspond to the standard deviation in boxes of $3 \times 3$ pixels centered on the respective pixel. The object numbers refer to the IDs in table 4.1. Note that the surface brightnesses are not corrected for cosmological $(1+z)^{4}$ dimming. 
MS98, spectroscopic redshifts were published by various authors for 61 of the analysed 522 galaxies, but here the basic aim was to test the diskmodel program, therefore the redshifts were not taken into account.

Note that the profiles presented in Fig. 4.11 are one-dimensional extractions, whereas the fits were two-dimensional. For each of the objects, the respective 1-D profiles along the $\mathrm{X}$ - and $\mathrm{Y}$ axis are shown, centered on the peak flux position. The $B / T$ ratios, as given in MS98, range from 0.00 to 0.09 (the median of the complete MS sample is $\langle B / T\rangle=0.07)$. At small projected distances from the galaxy centers, the PSF dominates the distribution of light and the observed profiles are flatter than would be expected for an exponential law. Moreover, it is evident from the plots that a bulge component was not detectable in the VLT simulation frames for any of these galaxies. The displayed errors $\sigma_{\mu}$ on the measured surface brightnesses were not derived via poisson statistics but calculated from $\sigma_{I}(x, y)$, as used in the $\chi^{2}$ computation (Eq. 4.7), according to

$$
\sigma_{\mu}=2.5 \log \left(\frac{I(x, y)+\sigma_{I}(x, y)}{I(x, y)}\right)
$$

The structural parameters for all galaxies in the MS sample are listed in table 4.1. The respective columns have the following meaning:

ID - Object number denoting the corresponding row in the full data table from MS98.

$i(\mathrm{HST}), r_{\mathrm{d}}(\mathrm{HST}), \theta(\mathrm{HST})-$ Disk inclination, apparent scale length and disk position angle as given in MS98, derived with GIM2D on the original drizzled HDF-N frames. The upper and lower $1 \sigma$ confidence limits are given separately. However, the errors are very symmetric, for the inclinations in particular. Since the errors were published rounded off, with two digits after decimal point, the scale length confidence limits of many galaxies are zero. The initial convention for the position angle used by MS98 referred to the $\mathrm{Y}$-axis instead of the $\mathrm{X}$-axis. To simplify the comparison, the pubished values $\theta_{\mathrm{MS} 98}$ have therefore been transformed to the SExtractor convention via $\theta=90^{\circ}-\theta_{\mathrm{MS} 98}$. The structural parameters of the bulge components (when detected with GIM2D), which were also published in MS98, were not considered with the exception of the $B / T$ ratio (see below).

$i(\mathrm{VLT}), r_{\mathrm{d}}(\mathrm{VLT}), \theta(\mathrm{VLT})$ - Structural parameters as derived with diskmodel on the convolved and rebinned HDF-N images. Due to the degraded spatial resolution, the errors are substantially larger than the GIM2D confidence limits. $B / T, I$ - Bulge-to-total ratio and total apparent brightness in the $\mathrm{F} 814 \mathrm{~W}$ filter as published by MS98. Since is has been shown at the beginning of this section that the transmission functions of the FORS $I$ and the F814W filters are very similar, the latter column is simply labelled "I".

$T$ - Morphological classification in the de Vaucouleurs scheme as given in vdB96. The authors considered only a fraction of the galaxies in the HDF-N and $46 \%$ of the 522 galaxies analysed in MS98. Thus, the type is given for only 24 of the listed objects.

A comparison of the structural parameters determined with GIM2D on the fully resolved images and by diskmodel on the rebinned and convolved images is shown in Fig. 4.12. Since the correlation between an observed rotation velocity $V_{\text {rot }}^{\mathrm{o}}$ and the intrinsic rotation velocity $V_{\text {rot }}^{\mathrm{i}}$ can roughly be approximated as

$$
V_{\mathrm{rot}}^{\mathrm{o}} \approx V_{\mathrm{rot}}^{\mathrm{i}} \sin i \cos \delta,
$$

where $\delta$ denotes the misalignment angle between disk major axis and slit direction, the inclination generally is the structural parameter with the strongest impact on the $V_{\max }$ derivation. Note that the influence of seeing, optical beam smearing and the scale length are neglected in this equation for the sake of simplicity. However, the rotation curve analysis accounted for all these effects. 
Table 4.1: The data of 39 galaxies from the HDF-N as given by Marleau \& Simard (1998) using GIM2D, compared to the results yielded by diskmodel on a simulated VLT image of the HDF-N. Note that the GIM2D errors on the scale lengths are less than 0.005 arcsec for many objects and the tabulated limits are 0.00 arcsec in those cases. See text for a description of the columns.

\begin{tabular}{|c|c|c|c|c|c|c|c|c|c|}
\hline ID & $\begin{array}{c}i(\mathrm{HST}) \\
{[\mathrm{deg}]}\end{array}$ & $\begin{array}{c}r_{\mathrm{d}}(\mathrm{HST}) \\
{[\operatorname{arcsec}]}\end{array}$ & $\begin{array}{c}\theta(\mathrm{HST}) \\
{[\mathrm{deg}]}\end{array}$ & $\begin{array}{c}i(\mathrm{VLT}) \\
{[\mathrm{deg}]}\end{array}$ & $\begin{array}{c}r_{\mathrm{d}}(\mathrm{VLT}) \\
{[\operatorname{arcsec}]}\end{array}$ & $\begin{array}{c}\theta(\mathrm{VLT}) \\
{[\mathrm{deg}]}\end{array}$ & $\mathrm{B} / \mathrm{T}$ & $\begin{array}{c}\mathrm{I} \\
{[\mathrm{mag}]}\end{array}$ & $\mathrm{T}$ \\
\hline 36 & $53.21_{-0.97}^{+0.97}$ & $0.12_{-0.00}^{+0.00}$ & $+49_{-1.8}^{+0.8}$ & $38 \pm 14$ & $0.20 \pm 0.03$ & $+51 \pm 12$ & 0.46 & 21.98 & \\
\hline 45 & $62.37_{-0.55}^{+0.55}$ & $0.39_{-0.00}^{+0.01}$ & $-54_{-0.59}^{+0.53}$ & $72 \pm 5$ & $0.50 \pm 0.06$ & $-51 \pm 9$ & 0.00 & 22.88 & 8 \\
\hline 48 & $73.42_{-0.09}^{+0.09}$ & $0.56_{-0.00}^{+0.00}$ & $-73_{-0.08}^{+0.06}$ & $74 \pm 3$ & $0.60 \pm 0.07$ & $-76 \pm 9$ & 0.00 & 21.42 & 4 \\
\hline 61 & $73.68^{7}$ & $0.65_{-0.01}^{+0.01}$ & $-78_{-0.19}^{+0.30}$ & $61 \pm 1$ & $0.59 \pm 0.02$ & $-77 \pm 4$ & 0.07 & 22.57 & 4 \\
\hline 72 & $51.55_{-}^{-}$ & $0.82_{-0.00}^{+0.00}$ & $\begin{array}{r}+21_{-0.02}^{+0.02} \\
\end{array}$ & $57 \pm 1$ & $0.92 \pm 0.01$ & $+22 \pm 4$ & 0.01 & 19.63 & \\
\hline 82 & $56.12_{-0.52}^{+0.52}$ & $0.22_{-0.00}^{+0.00}$ & $-82_{-0.46}^{+0.59}$ & $57 \pm 5$ & $0.25 \pm 0.04$ & $-83 \pm 16$ & 0.27 & 21.68 & 3 \\
\hline 85 & $31.30_{-1.21}^{+1.21}$ & $0.17_{-0.00}^{+0.00}$ & $\begin{array}{r}+0.00 \\
+50_{-2.17}^{+2.04}\end{array}$ & $20 \pm 20$ & $0.18 \pm 0.04$ & $-16 \pm 15$ & 0.00 & 22.97 & \\
\hline 117 & $58.96_{-0.16}^{+0.16}$ & $0.37_{-0.00}^{+0.000}$ & $-40_{-0.17}^{+0.14}$ & $52 \pm 5$ & $0.30 \pm 0.02$ & $-39 \pm 20$ & 0.00 & 21.07 & \\
\hline 126 & $78.74_{-0.14}^{+0.16}$ & $0.34_{-0.00}^{+0.00}$ & $\begin{array}{r}-0.17 \\
+86_{-0.22}^{+0.16}\end{array}$ & $65 \pm 1$ & $0.29 \pm 0.03$ & $+85 \pm 7$ & 0.00 & 22.94 & 1 \\
\hline 130 & $58.17_{-1.05}^{+1.05}$ & $0.17_{-0.00}^{+0.00}$ & $\begin{array}{r}+55_{-1.22}^{+1.42} \\
\end{array}$ & $47 \pm 10$ & $0.19 \pm 0.03$ & $+20 \pm 9$ & 0.52 & 22.73 & 8 \\
\hline 150 & $48.03_{-0.56}^{+0.56}$ & $0.30_{-0.00}^{+0.00}$ & $-95_{-0.61}^{+0.50}$ & $50 \pm 10$ & $0.26 \pm 0.01$ & $-87 \pm 23$ & 0.00 & 22.32 & 4 \\
\hline 175 & $79.77_{-0.15}^{+0.15}$ & $0.55_{-0.00}^{+0.00}$ & $\begin{array}{r}-0.61 \\
+23_{-0.18}^{+0.15}\end{array}$ & $80 \pm 2$ & $0.68 \pm 0.08$ & $+20 \pm 5$ & 0.00 & 22.86 & 4 \\
\hline 198 & $75.68_{-0.30}^{+0.150}$ & $0.26_{-0.00}^{+0.00}$ & $-80_{-0.1}^{+0.1}$ & $59 \pm 5$ & $0.25 \pm 0.04$ & $+87 \pm 3$ & 0.12 & 22.45 & 8 \\
\hline 212 & $39.76_{-1.41}^{+1.41}$ & $0.48_{-0.01}^{+0.00}$ & $\begin{array}{r}-03_{-2.65}^{+2.19} \\
-\end{array}$ & $49 \pm 8$ & $0.51 \pm 0.04$ & $-65 \pm 27$ & 0.00 & 22.94 & \\
\hline 226 & $43.32_{-0.19}^{+0.41}$ & $0.41_{-0.00}^{+0.01}$ & $\begin{array}{r}-2.65 \\
+47_{-0.30}^{+0.27}\end{array}$ & $39 \pm 11$ & $0.35 \pm 0.04$ & $+39 \pm 12$ & 0.09 & 20.87 & \\
\hline 239 & $69.92_{-0.48}^{+0.48}$ & $0.22_{-0.00}^{+0.00}$ & $-17_{-0.42}^{+0.40}$ & $59 \pm 9$ & $0.28 \pm 0.02$ & $-25 \pm 6$ & 0.35 & 22.24 & 3 \\
\hline 255 & $42.42_{-1.30}^{+1.40}$ & $0.33_{-0.00}^{+0.00}$ & $+85_{-0.66}^{+0.43}$ & $33 \pm 14$ & $0.27 \pm 0.03$ & $+80 \pm 12$ & 0.49 & 21.80 & 4 \\
\hline 266 & $76.04_{-020}^{+0.20}$ & $0.32_{-0.00}^{+0.00}$ & $-78_{-026}^{+0.19}$ & $77 \pm 4$ & $0.29 \pm 0.05$ & $-80 \pm 9$ & 0.00 & 22.71 & 2 \\
\hline 271 & $71.76_{-000}^{+0.02}$ & $1.35_{-0.00}^{+0.00}$ & $-48_{-0.02}^{+0.08}$ & $71 \pm 1$ & $1.45 \pm 0.05$ & $-48 \pm 3$ & 0.26 & 20.00 & \\
\hline 279 & $71.43_{-0.10}^{+0.10}$ & $0.29_{-0.00}^{+0.00}$ & $+60_{-0.11}^{+0.13}$ & $54 \pm 5$ & $0.24 \pm 0.04$ & $+65 \pm 24$ & 0.00 & 21.49 & 2 \\
\hline 298 & $42.37_{-0.66}^{+0.10}$ & $0.38_{-0.00}^{+0.00}$ & $-42_{-1.07}^{+0.89}$ & $46 \pm 8$ & $0.38 \pm 0.06$ & $-55 \pm 9$ & 0.00 & 22.30 & 4 \\
\hline 299 & $65.51_{-1.10}^{+1.66}$ & $0.49_{-0.02}^{+0.00}$ & $-22_{-1.07}^{+1.07}$ & $60 \pm 6$ & $0.34 \pm 0.01$ & $-30 \pm 12$ & 0.53 & 22.73 & \\
\hline 336 & $33.62_{-0.98}^{+0.10}$ & $0.30_{-0.00}^{+0.02}$ & $-68_{-1.37}^{+1.07}$ & $38 \pm 19$ & $0.26 \pm 0.02$ & $-70 \pm 18$ & 0.00 & 22.22 & 8 \\
\hline 350 & $74.61_{-0.42}^{+0.98}$ & $\begin{array}{l}0.37_{-0.00}^{+0.00} \\
+0.00\end{array}$ & $\begin{array}{r}-1.37 \\
+23_{-0.35}^{+0.23}\end{array}$ & $65 \pm 6$ & $0.32 \pm 0.01$ & $+25 \pm 12$ & 0.25 & 22.62 & 3 \\
\hline 359 & $44.21_{-1.13}^{+1.13}$ & $0.57_{-0.01}^{+0.00}$ & $\begin{array}{r}+0.55 \\
+36_{-1.56}^{+1.57}\end{array}$ & $54 \pm 1$ & $0.72 \pm 0.04$ & $+30 \pm 15$ & 0.00 & 22.51 & 5 \\
\hline 368 & $54.10_{-1.01}^{+1.01}$ & $0.24_{-0.00}^{+0.00}$ & $\begin{array}{r}-1.26 \\
+37_{-1.46}^{+1.28}\end{array}$ & $52 \pm 12$ & $0.22 \pm 0.01$ & $+35 \pm 24$ & 0.22 & 22.81 & 3 \\
\hline 398 & $64.51_{-0.18}^{+0.01}$ & $0.45^{+0.00}$ & $-10^{-0.24}$ & $65 \pm 5$ & $0.42 \pm 0.06$ & $-10 \pm 15$ & 0.00 & 20.96 & \\
\hline 399 & $36.34_{-0.50}^{+0.18}$ & $0.32_{-0.00}^{+0.000}$ & $\begin{array}{r}-0.24 \\
-34_{-0.25}^{+0.39}\end{array}$ & $40 \pm 11$ & $0.30 \pm 0.02$ & $-24 \pm 25$ & 0.08 & 21.51 & \\
\hline 400 & $64.61_{-0.54}^{+0.50}$ & $0.68_{-0.01}^{+0.00}$ & $\begin{array}{r}-0.25 \\
-1_{-0.48}^{+0.35}\end{array}$ & $70 \pm 4$ & $0.50 \pm 0.05$ & $-10 \pm 12$ & 0.03 & 22.04 & \\
\hline 415 & $38.16_{-0.86}^{+0.54}$ & $0.10_{-0.00}^{+0.01}$ & $-73_{-0}^{+0}$ & $29 \pm 20$ & $0.15 \pm 0.03$ & $-98 \pm 78$ & 0.47 & 21.84 & \\
\hline 431 & $55.05_{-0.15}^{+0.15}$ & $0.66_{-0.00}^{+0.00}$ & $-8_{-0.38}^{+0.34}$ & $54 \pm 4$ & $0.92 \pm 0.05$ & $+15 \pm 6$ & 0.07 & 21.31 & \\
\hline 440 & $31.99_{-200}^{+2.02}$ & $0.35_{-0.01}^{+0.00}$ & $-53_{-}^{+}$ & $30 \pm 16$ & $0.29 \pm 0.04$ & $-85 \pm 30$ & 0.08 & 22.85 & 4 \\
\hline 441 & $43.26_{-0.94}^{+0.94}$ & $0.17_{-0.00}^{+0.00}$ & $+3_{-1}^{+1}$ & $50 \pm 10$ & $0.17 \pm 0.01$ & $+15 \pm 36$ & 0.17 & 22.60 & 3 \\
\hline 469 & $58.75_{-0.53}^{+0.93}$ & $0.12_{-0.00}^{+0.000}$ & $+3_{-0}^{+0}$ & $45 \pm 13$ & $0.20 \pm 0.03$ & $+20 \pm 18$ & 0.17 & 22.89 & \\
\hline 479 & $45.09_{-1.07}^{+-1.53}$ & $0.39_{-0.01}^{+0.00}$ & $\begin{array}{r}-0.63 \\
+33_{-1.10}^{+0.82}\end{array}$ & $50 \pm 9$ & $0.31 \pm 0.01$ & $+35 \pm 21$ & 0.15 & 22.30 & 4 \\
\hline 514 & $76.31_{-0.24}^{+0.07}$ & $0.54_{-0.00}^{+0.01}$ & $\begin{array}{r}-1.10 \\
+76_{-0.21}^{+0.22}\end{array}$ & $75 \pm 1$ & $0.66 \pm 0.05$ & $+80 \pm 9$ & 0.00 & 22.51 & 2 \\
\hline 518 & $66.91_{-0.89}^{+0.24}$ & $0.26_{-0.00}^{+0.00}$ & $\begin{array}{r}-0.21 \\
+50_{-0.32}^{+0.31}\end{array}$ & $54 \pm 8$ & $0.29 \pm 0.06$ & $+55 \pm 15$ & 0.46 & 22.43 & 3 \\
\hline 520 & $47.51_{-0.63}^{+0.69}$ & $0.33_{-0.00}^{+0.00}$ & $-51_{-0.67}^{+0.52}$ & $43 \pm 12$ & $0.30 \pm 0.04$ & $-55 \pm 12$ & 0.00 & 22.11 & 8 \\
\hline 521 & $55.82_{-0.06}^{+0.03}$ & $0.56_{-0.00}^{+0.00}$ & $-60_{-0.06}^{+0.08}$ & $55 \pm 1$ & $0.62 \pm 0.01$ & $-70 \pm 6$ & 0.00 & 19.91 & \\
\hline
\end{tabular}



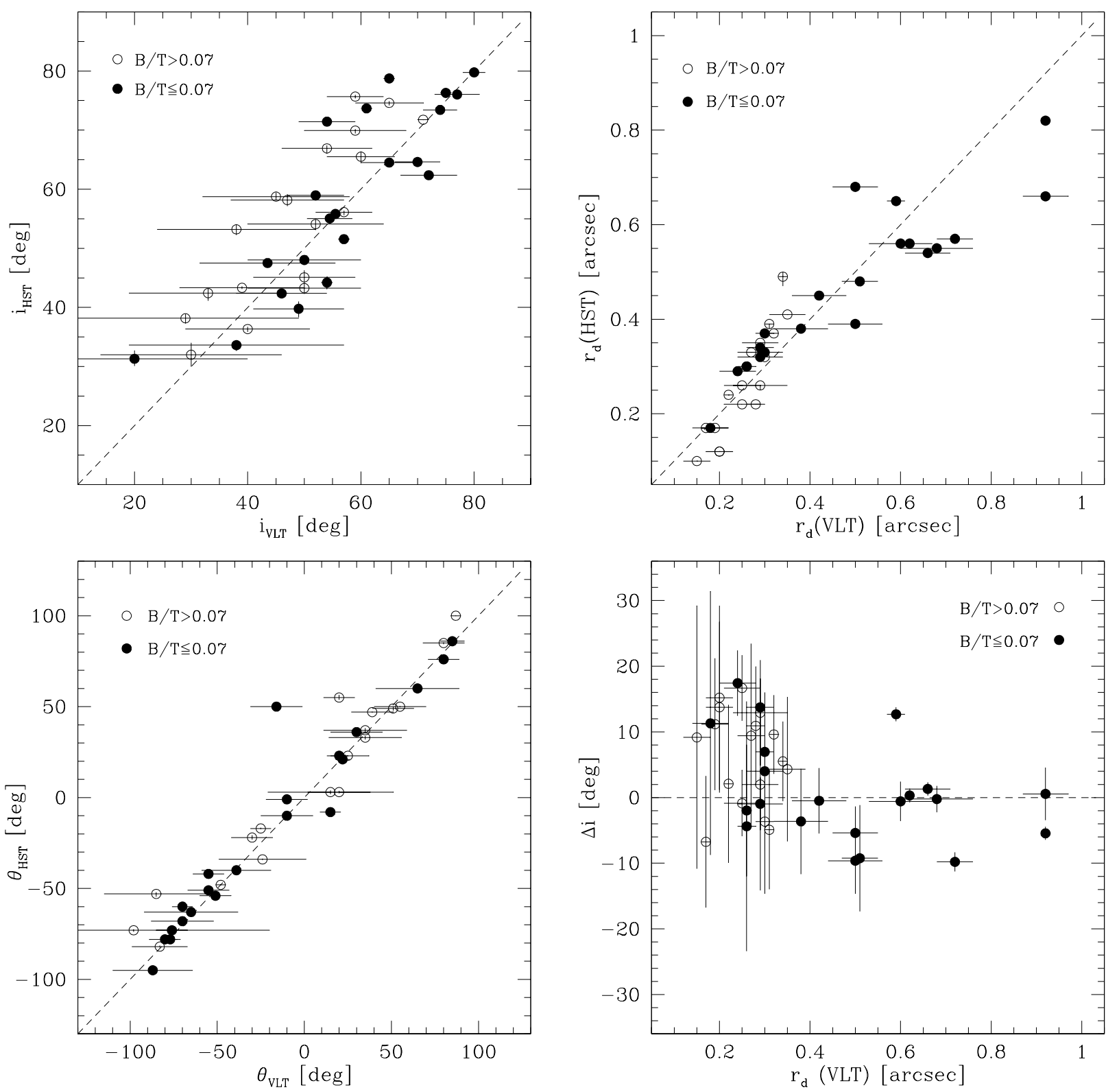

Figure 4.12: Comparison of the structural parameters of 39 galaxies from the HDF-N as derived with GIM2D by Marleau \& Simard (1998) on the original drizzled images (label "HST") and with diskmodel on the VLT simulation of the HDF-N (label "VLT"). The upper left, upper right and lower left plots show the comparison of the disk inclinations, scale lengths and position angles, respectively. In the lower right figure, the difference $\Delta i=i_{\mathrm{HST}}-i_{\mathrm{VLT}}$ is plotted against the scale lengths derived with the simulated VLT frames. The sample is sub-divided according to the bulge-to-total ratio given by Marleau \& Simard: 20 Galaxies exceeding the median $B / T$ of the data set (i.e., $B / T>0.07$ ) are denoted by open circles, 19 galaxies with $B / T \leq 0.07$ are displayed as filled circles. For the majority of the objects, the structural parameters agree within the errors. However, a slight underestimation of the diskmodel-inclinations for galaxies with significant bulge components can be deduced. This also is the case for spirals with very small scale lengths (lower right figure). See text for details. 
Therefore, the upper left plot in Fig. 4.12 is particularly important, where the inclinations $i_{\mathrm{HST}}$ derived from space-based and $i_{\mathrm{VLT}}$ from (simulated) ground-based HDF-N imaging are compared to each other. Two conclusions can be drawn from this figure. Firstly, the errors of the diskmodel fits are much larger, which was to be expected as an effect of the substantially lower spatial resolution. And secondly, for spirals with significant bulges, the $i_{\mathrm{HST}}$ values are on the mean slightly larger than the $i_{\mathrm{VLT}}$ values. This can be also deduced from Fig. 4.13, where the discrepancy $\Delta i=i_{\mathrm{HST}}-i_{\mathrm{VLT}}$ is shown as a function of the $B / T$ ratio. For the purpose of this graph, the 17 spirals without any detectable bulge component, i.e. $B / T=0.0$ (17 out of $39)$, are denoted by their median of $\Delta i$ and the mean error. In the complete MS sample, the ground-based fit values underestimate the inclinations in the median by only $1.3^{\circ}$. When the 7 galaxies with strong bulges $(B / T>0.3)$ are neglected, the agreement is excellent $\left(\langle\Delta i\rangle<0.1^{\circ}\right)$. Only spirals of very early morphological type, i.e. large bulge-to-total ratios, show a significant systematic error in their inclinations which amounts to $\sim 10^{\circ}$.

The lower right plot in Fig. 4.12 indicates that $\Delta i$ is also enlarged for spirals with very small VLT-based scale lengths $r_{\mathrm{d}}<0.3$ arcsec. Here, the median is $\langle\Delta i\rangle=9.4^{\circ}$, whereas for larger disks, the discrepancy is negligible, $\langle\Delta i\rangle<0.1^{\circ}$. Note that a small median does not imply here that the typical absolute deviations are small. However, since it was the aim of the simulation to verify whether the inclinations could introduce a systematic error in the upcoming TF analysis, a systematic under- or overestimation has to be tested. For this purpose, the median of $\Delta i$ is the appropriate quantity.

Hence, these two graphs imply that the onecomponent fits on ground-based images may lead to slight systematic underestimations of the inclinations of FDF spirals with a) bulges that would be detectable on HST frames but are smoothed out on VLT frames or b) very small

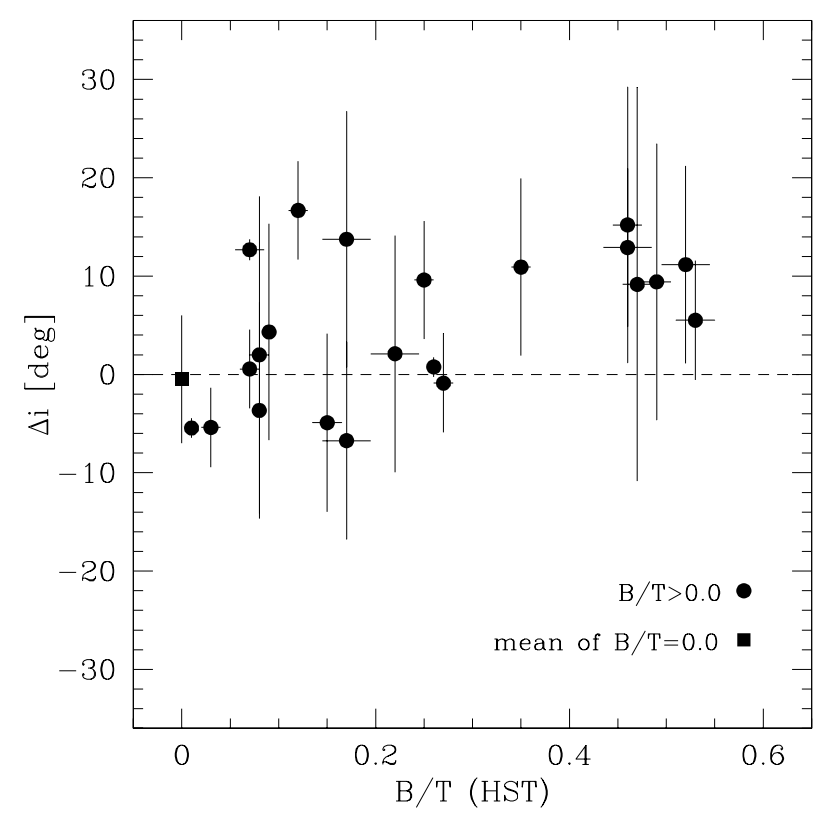

Figure 4.13: Deviations $\Delta i$ between the HSTbased inclinations given by Marleau \& Simard (1998) and the simulated ground-based inclinations as a function of the bulge-to-total ratio. For the 17 spirals without a detectable bulge $(\mathrm{B} / \mathrm{T}=0.0)$, the median $\Delta i$ and the mean error are shown.

apparent scale lengths.

However, for a couple of reasons it is very unlikely that a significant systematic effect on the $\mathrm{TF}$ analysis could be induced by this.

Firstly, as has been already shown in the last section, the inclinations of the FDF TF sample are independent of type. It should therefore be improbable that a fraction of the early-type spirals exhibit strong bulges.

Secondly, the apparent scale lengths of only three TF spirals with high quality rotation curves (on which the TF analysis will be based) are smaller than 0.3 arcsec. The trend towards larger values of $\Delta i$ for very small scale lengths should therefore be negligible.

And thirdly, one has to keep in mind that even the underestimation of $\sim 10^{\circ}$ for spirals with strong bulges would have a relatively small systematic effect on the intrinsic $V_{\max }$. At a VLTbased inclination of $50^{\circ}$, which is roughly the 
median in the TF sample, the corresponding systematic error in $V_{\max }$ is $\sim 13 \%$, and the TF offset induced by this (assuming a TFR slope of -7.5) is $\sim 0.4$ mag. A potential impact of the groundbased imaging on the results from the TF analysis will be further discussed in chapter 6.4.7.

The upper right plot in Fig. 4.12 indicates an underestimation of the scale length for relatively large and very small disks $\left(r_{\mathrm{d}}<0.2\right.$ arcsec $)$. For the vast majority of the MS sample galaxies, however, the agreement is very good. The median discrepancy for all 39 spirals is $<0.1 \%$.

The third structural parameter that is used in the following $\mathrm{TF}$ analysis, the position angle, could also be well determined on the simulated VLT frames. 28 out of the 39 MS galaxies agree with the GIM2D values to within $10 \%$, and 34 to within $20 \%$. Note that the latter constraint corresponds to a systematic error in $V_{\max }$ of less than $7 \%$ (see Eq. 4.17). Neither the scale lengths nor the position angles derived with diskmodel show a dependence on the HST-based $B / T$ ratios.

\subsection{Morphologies}

As pointed out in chapter 4.2 , the sub-types of the FDF galaxies were derived on the basis of their SEDs. The resolution provided by the ground-based images was not sufficient for a morphological classification according to the Hubble scheme. However, a framework of morphological types was introduced by Abraham et al. (1996, A96 hereafter) which is reliable for imaging data at resolutions down to $\sim 100$ usable pixels per object. This is the minimum number of resolution elements per galaxy that is achieved for the FDF spirals in the $I$ reference frame, even if a lower limit of $>2 \sigma$ sky noise is set. The scheme of A96 will now be briefly described.

Their approach of a morphological classification is, in contrast to the original Hubble "tuning fork", purely quantitative. It relies on two parameters, the so-called asymmetry index $A$ and the concentration index $C$. While the latter is sensitive to the steepness of a galaxies profile, the former yields the deviation of the profile from symmetry.

For the computation of the concentration index, a galaxies flux $2 \sigma$ above the sky noise within an elliptical area $G_{1}$ is compared to the flux within an area $G_{\alpha}$ of smaller normalized radius $\alpha$ :

$$
C=\frac{\sum_{i, j \in G_{\alpha}} I_{i j}}{\sum_{k, l \in G_{1}} I_{k l}}
$$

where $I_{i j}$ is the flux at the pixel $(i, j)$ within the inner isophotal region $G_{\alpha}$ and $I_{k l}$ is the flux at the pixel $(k, l)$ within the larger isophotal region $G_{1}$. In other words, the higher the value of $C$, the more the light profile is concentrated towards small galactocentric radii. Since the de Vaucouleurs profile of an elliptical galaxy is steeper in the inner part than the exponential disk profile of spirals, the concentration index can be used to discriminate between these two types. A96 found that a value of $\alpha=0.3$ yields the best discriminating power, and this factor was adopted here also.

For the measurement of the asymmetry index $A$, the galaxy image is rotated by $180^{\circ}$, then subtracted from the original image and normalized to its flux via

$$
A=\frac{\sum_{i, j}\left|I_{i j}-I_{i j}^{180}\right|}{\sum_{i, j} I_{i j}} .
$$

Here, $I_{i j}$ is the flux at the pixel $(i, j)$ in the original image and $I_{i j}^{180}$ the flux at the pixel $(i, j)$ in the rotated image. The asymmetry index can be used in particular to discriminate between spirals and irregulars. Since absolute values of the difference in flux are summed up, the sky noise has to be taken into account. For the measurements on the simulated HDF frames and the FDF reference frame, this was achieved by neglecting all positions $(i, j)$ where

$$
\left|I_{i j}-I_{i j}^{180}\right|<\sigma_{\text {sky }},
$$

i.e., no addends smaller than the sky noise contributed to the right hand side of Eq. 4.19. 
Another critical point was the definition of the rotation axis. Since the Source Extractor was used to define the central position of each object prior to the computation of $A$ and $C$, it was mandatory to verify that this "central pixel" (which was computed by SExtractor as the first order moment of the profile) coincided with the axis of highest symmetry. This was done by automatically generating a set of 25 rotated galaxy images which were shifted with respect to the original image by up to \pm 2 pixel in each direction along the $\mathrm{X}$ and $\mathrm{Y}$ axes, respectively. For each version of the rotated image, $A$ then was computed via Eq. 4.19 with the constraint defined in Eq. 4.20. The minimum of the resulting 25 values of $A$ was adopted as the most accurate one.

Basically, the additional computation step of small integer pixel shifts was performed to account for the large pixel scale of the VLT frames with respect to the HST images. Moreover, a "straightforward" derivation of $A$ with Eq. 4.19 yielded overestimated asymmetries and a very weak distinction between E/S0, spiral and irregular galaxies in $A$-space. Published values for $A$ and $C$ in the HDF-N have been used as a reference here, see further below.

When an ensemble of galaxies is inspected in logarithmic $A / C$-space, it is found that E/S0 galaxies, spirals and irregulars populate three separated regions. To derive the discriminating lines between the different sub-types, A96 used a data set of local galaxies from Frei et al. (1996) with visually derived morphologies. First, A96 simulated how images of these galaxies would be affected if they were located at high redshifts. Then, $A$ and $C$ indices of these artificially redshifted objects were computed, yielding a linear distinction between irregulars and spirals, and another line which separated spirals from E/S0 galaxies.

To gain a re-calibration of these lines for the ground-based imaging data, the values of $A$ and $C$ as computed in the HDF-N by vdB96 were used as a reference. These values are compared to the authors' visual classification for 60 relatively bright $\left(I<23.5^{m}\right)$ galaxies in the upper panel of Fig. 4.14. All of these objects are also part of the MS98 sample. The two discriminating lines are shown as derived by A96. Though 3 irregular and $3 \mathrm{E} / \mathrm{S} 0$ galaxies populate "wrong" regions of the $A / C$ diagram, the three zones of the different morphological types are clearly revealed. Irregular galaxies are characterised by high values of $A$ and low values of $C$, spirals feature intermediate asymmetries and concentration indices, whereas $\mathrm{E} / \mathrm{S} 0$ galaxies on the mean have the smallest values of $A$ and the highest values of $C$. Note that the presence of a small fraction of the spirals in the region of early-type galaxies indicates strong bulge components in these objects.

In the lower graph of Fig. 4.14, the values of $A$ and $C$ for the same sample, but as derived on the VLT simulation of the HDF-N, are presented. The symbols again denote the visual classification by vdB96. Except for a few more E/S0 galaxies which are now located in the "spiral region", the distinction between the three sub-types is as robust as based on the original HDF frames. In particular, the mean and the scatter in $A$ and $C$ are very similar between the space-based and the (simulated) groundbased approach. The respective values of the 32 spiral galaxies are $\overline{C_{\mathrm{HST}}}=0.405 \pm 0.105$, $\overline{C_{\mathrm{VLT}}}=0.405 \pm 0.106, \overline{A_{\mathrm{HST}}}=0.130 \pm 0.070$ and $\overline{A_{\mathrm{VLT}}}=0.124 \pm 0.068$. The $18 \mathrm{E} / \mathrm{S} 0$ galaxies yielded values of $\overline{C_{\mathrm{HST}}}=0.580 \pm 0.097$, $\overline{C_{\mathrm{VLT}}}=0.533 \pm 0.094, \overline{A_{\mathrm{HST}}}=0.097 \pm 0.067$ and $\overline{A_{\mathrm{VLT}}}=0.093 \pm 0.039$. Finally, the 10 irregular galaxies (visually classified as Sdm or later by vdB96) have respective mean indices of $\overline{C_{\mathrm{HST}}}=0.325 \pm 0.081$, $\overline{C_{\mathrm{VLT}}}=0.321 \pm 0.104, \overline{A_{\mathrm{HST}}}=0.199 \pm 0.093$ and $\overline{A_{\mathrm{VLT}}}=0.273 \pm 0.124$. With the exception of slightly overestimated asymmetry indices for irregulars, the agreement is excellent.

The above comparison provided a strong ar- 

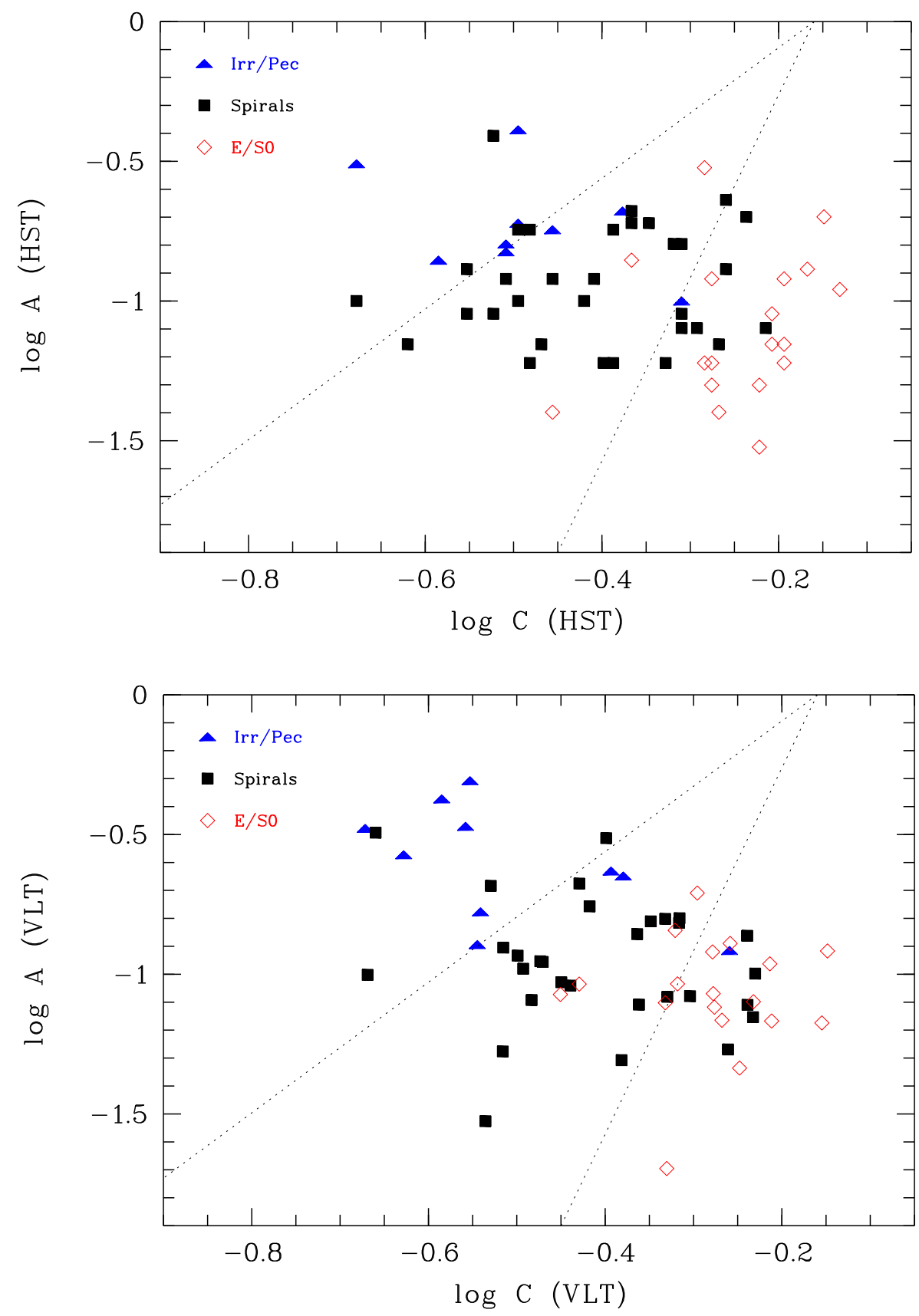

Figure 4.14: Upper panel: Asymmetry index $A$ and concentration index $C$ of 60 galaxies with $I<23.5^{m}$ as measured in the original HDF-N images by van den Bergh et al. (1996). The visual classification performed by the authors is illustrated with different symbols. The two dotted lines give the discriminating lines between irregular and spiral morphology (upper line) and spiral and E/S0 morphology (lower line) as defined by Abraham et al. (1996). Lower panel: Same subsample, but asymmetry and concentration indices here were derived from the simulated VLT images of the HDF-N. The distinction between spiral and early-type morphology is only slightly less robust than in the original HDF-N frames. 
gument for the usability of VLT images with 0.5 arcsec FWHM for accurate measurements of $A$ and $C$ in the regime $I<23.5^{m}$ and hence for the entire FDF sample (the faintest spiral covered by the MOS observations had $I=23.15^{m}$, the faintest spiral which could be used for the $V_{\text {max }}$ derivation had $\left.I=22.97^{m}\right)$. However, the distribution in $A / C$-space shown in Fig. 4.14 (lower panel) indicated that the two discriminating lines had to be slightly redefined at groundbased resolution.

To further test this, a visual inspection of the morphologies of 48 relatively bright $\left(I<21.5^{m}\right)$ TF spirals was performed. In this by-eye approach, the objects were either classified as spirals (if they showed regular isophotes) or irregular/peculiar galaxies. An additional subset of $26 \mathrm{E} / \mathrm{S} 0$ galaxies from the FDF was selected on the SED basis with the same brightness constraint. It was verified that all these objects had no nearby companions to prevent any overestimated asymmetry indices. In the upper graph of Fig. 4.15, the $A / C$ diagram of these 74 galaxies is shown and compared to the visual classification of the spirals/irregulars and the spectral classification of the E/S0 galaxies.

On the basis of this distribution in $A / C$ space, it was decided to shift the discriminating lines from their A96 definition (which is indicated by the dotted lines) to the positions denoted by the dashed lines. This new ground-based definition features a slightly lower boundary value between spirals and $\mathrm{E} / \mathrm{S} 0$ galaxies in $C$-space and also a shallower line separating spirals and irregulars. With these redefined zones of the $A / C$ diagram, the potential connection between morphological and spectral type - well known from the Hubble scheme of local galaxies - will now be considered.

In the lower panel of Fig. 4.15, the $A$ and $C$ indices of $74 \mathrm{TF}$ objects and 26 early-type FDF galaxies are shown. Three spirals and four earlytype galaxies from the full sample were rejected for this purpose because their aymmetry indices were affected by very close companions in the line-of-sight. Note that the symbols here solely denote the SED type, either E/S0, Sab, Sc or $\mathrm{Sdm} / \mathrm{Im}$. Two important conclusions can be drawn from this plot:

- For the majority of the objects, the SED type corresponds to the morphological type. 7 out of $20 \mathrm{Sdm} / \mathrm{Im}$ galaxies (35\%), 15 out of $41 \mathrm{Sc}$ spirals (37\%), 2 out of $13 \mathrm{Sab}$ spirals $(15 \%)$ and 8 out of 26 early-type galaxies $(31 \%)$ populate "wrong" regions in $A / C$ space. In particular, only roughly one third of the spirals with intermediate-type SEDs feature a very late-type morphology and vice versa.

- Only five spirals (corresponding to $\sim 7 \%$ of the TF sample) probably have a strong bulge component since they cover the region of $\mathrm{E} / \mathrm{S} 0$ morphology. Four of these have SEDs of type Sc.

It is tempting to conclude that the basic Hubble scheme, i.e., a correlation between morphological and spectral type, already was present at a redshift of $z \approx 0.5$, where the universe had $\sim 65 \%$ of its current age. The third aspect for the distinction between different galaxy types are the kinematics. Early-type galaxies are dynamically hot and are stabilized due to random motions (e.g., Bender et al. 1992), whereas spirals are dynamically cold, which means these systems are stabilized due to ordered rotation.

To use the kinematics as a third classification criterion in addition to the SED and morphological types, it is interesting to examine the $A / C$ diagram of galaxies which did not yield a value for the maximum rotation velocity. Mostly this was due to asymmetric or disturbed rotation curves, in fewer cases because of too low an $\mathrm{S} / \mathrm{N}$ or geometric distortions, i.e., too large misalignment angles $\delta$ or too low inclinations $i$ - see chapter 5.2.2 for a more detailed description of this topic. 

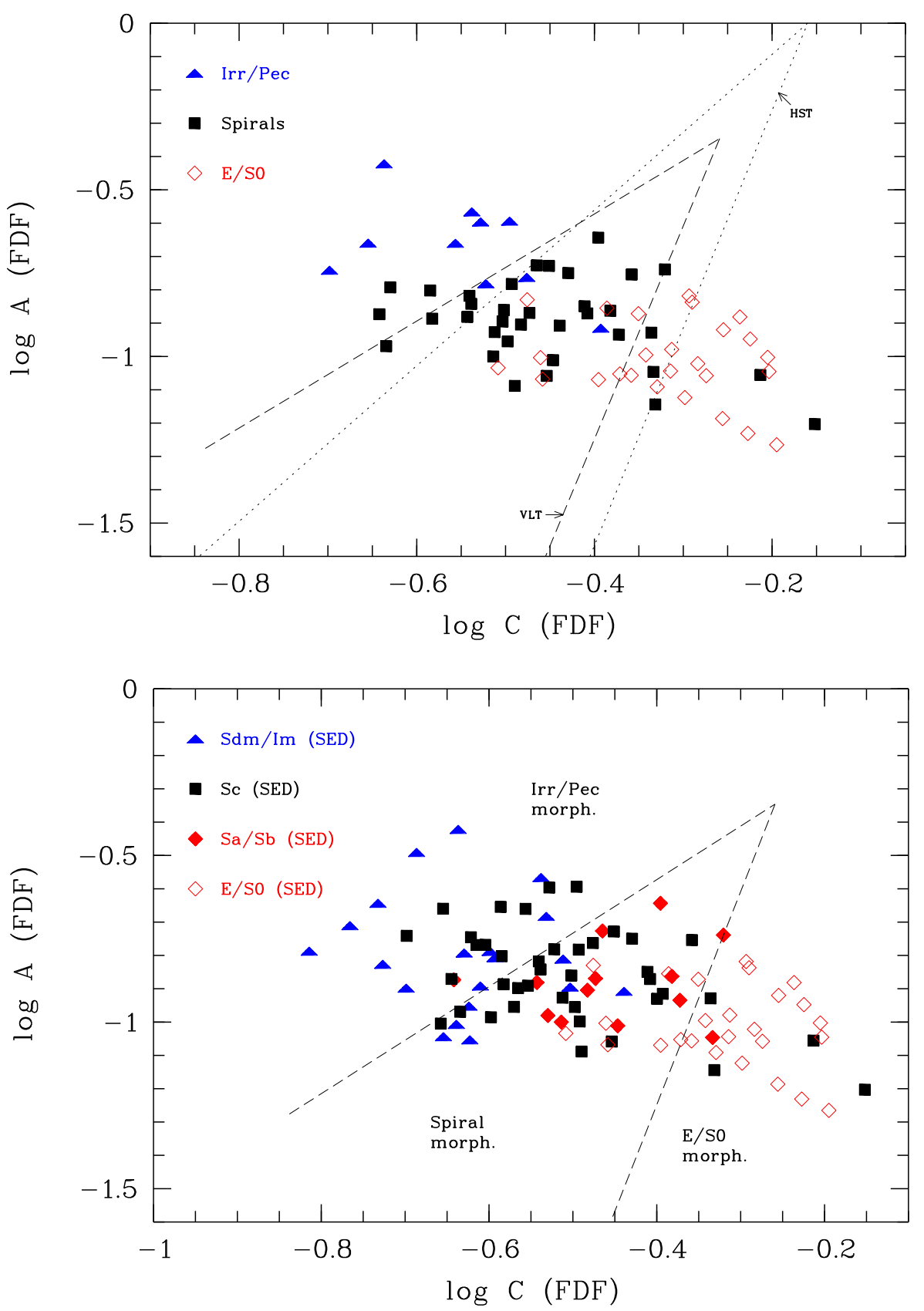

Figure 4.15: Upper panel: VLT-based asymmetry and concentration indices of 78 galaxies with $I<21.5^{m}$ from the FDF. Of these, 48 galaxies from the TF sample have been visually classified as spirals or of irregular/peculiar morphology. The 26 early-type galaxies included have been selected purely on the basis of their SED types. The two dotted lines indicate the discriminating lines between irregular, spiral and early-type morphology as defined by Abraham et al. (1996) using the original HDF images. The two dashed lines indicate slightly modified boundaries to account for the lower spatial resolution in the FDF reference frame. Lower panel: Asymmetry and concentration indices of 74 spirals in the TF sample and of 26 earlytype FDF galaxies. Contrary to the upper graph, the symbols here denote the SED types of all objects. For the majority of the galaxies, spectral and morphological type are correlated. Only five of the spirals populate the $A / C$ region of early-type galaxies, indicating strong bulge components in these galaxies' profiles. 


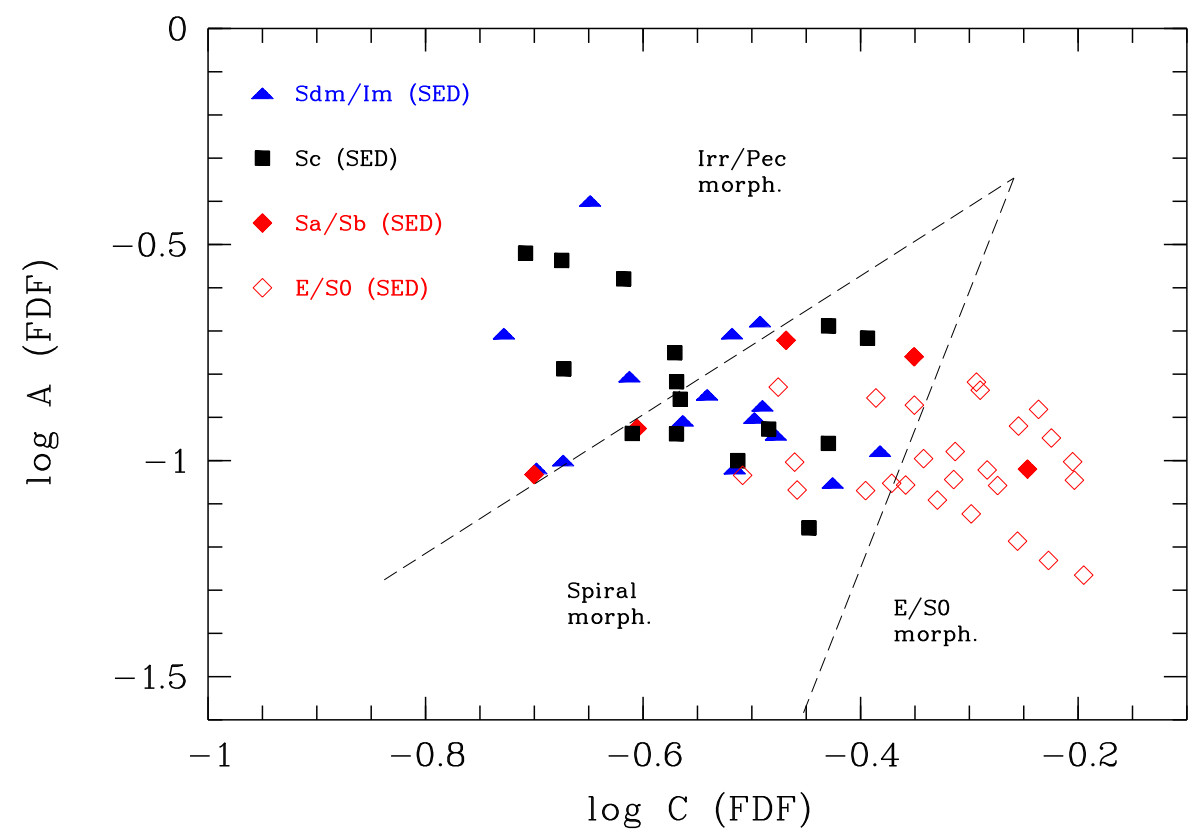

Figure 4.16: VLT-based asymmetry and concentration indices of 35 FDF spirals which could not be used for the derivation of $V_{\max }$, mainly due to peculiar/asymmetric rotation curves. 26 early-type FDF galaxies are additionally shown for comparison. The correlation between SED type (indicated by the different symbols) and the position in $A / C$-space is weakend with respect to the TF sample shown in the bottom panel of Fig. 4.15. Only one of the non-TF spirals features a concentration index that indicates a strong bulge component.

These non-TF galaxies are shown in Fig. 4.16. In total, 35 late-type galaxies were not appropriate for a $V_{\max }$ derivation, 26 early-type galaxies are shown as a comparison. One non-TF spiral was rejected here due to a nearby companion. The correlation between morphology and SED type is slightly weaker than for the TF objects. 7 out of 15 galaxies (47\%) with spectral type Sdm or later show an irregular morphology, and 8 out of 14 Sc spirals ( $57 \%$ ) populate the regime of regular spirals in $A / C$ space. If Sab's are included, 11 out of 19 spirals earlier than Sc (58\%) show a correlation. Three out of the five Sab's are located along the discriminating line between spirals and irregulars, in contrast to the TF Sab galaxies.

On the one hand, the fraction of galaxies with irregular morphology (41\%) is slightly larger than for the kinematically unpeculiar objects from the $\mathrm{TF}$ sample (32\%). On the other hand, the mean asymmetry index and its scatter are only marginally increased: the 35 non-TF spirals/irregulars have $\bar{A}=0.155 \pm 0.072$, the $74 \mathrm{TF}$ galaxies feature $\bar{A}=0.151 \pm 0.056$. Therefore, it cannot be deduced from the asymmetry indices of the galaxies which could not be used for a derivation of $V_{\max }$ that their peculiar kinematics coincide with morphological imprints, that is, peculiar light profile shapes.

Nevertheless, two major conclusions can be drawn from this section: a) The kinematically unpeculiar FDF spirals show a correlation between SED type and morphology similar to the Hubble scheme of local galaxies and b) only a very small fraction $(\sim 7 \%)$ of the TF spirals show $A / C$ indices indicating a strong bulge component, yielding further arguments for the reliability of the one-component surface brightness profile fits. This topic will be referred to again as a test of the TF analysis in chapter 6.4.7. 


\subsection{Luminosity Derivation}

As stated before, the computation of the restframe absolute magnitudes of the FDF spirals benefitted extensively from the deep multi-band imaging. The apparent brightness of a given object was derived from the filter $X$ which best matched the $B$-band response curve in restframe. According to the redshift of a certain galaxy, this filter $X$ was either the $B$-, $g$-, $R$ - or $I$-band (see section 4.6.4). The adopted equation for the computation of absolute $B$ magnitudes was

$$
\begin{aligned}
M_{B}= & m_{X}-A_{X}^{g}-D M_{\Lambda}\left(z, H_{0}\right) \\
& -k_{B}(X, T, z)-A_{B}^{i} .
\end{aligned}
$$

Here, the respective terms have the following meaning:

$m_{X}$ is the apparent total brightness in the passband $X$ as derived with the Source Extractor (see section 4.6.1).

$A_{X}^{g}$ is the Galactic absorption in the wavelength regime of filter $X$ (see section 4.6.2).

$D M_{\Lambda}\left(z, H_{0}\right)$ is the distance modulus for the respective redshift. As stated earlier, the so-called concordance cosmology (e.g., Spergel et al. 2003) is adopted here and throughout this thesis, i.e. a flat universe with a matter density $\Omega_{m}=0.3$, a cosmological constant corresponding to an energy density of $\Omega_{\Lambda}=0.7$ and a Hubble constant of $H_{0}=70 \mathrm{~km} \mathrm{~s}^{-1} \mathrm{Mpc}^{-1}$. A potential impact of the adopted cosmology on this study will be briefly discussed in section 4.6.3.

$k_{B}(X, T, z)$ is the $k$-correction for the transformation from filter $X$ to the rest-frame $B$ band which accounts for i) the different response curves of filter $X$ in observer's frame and filter $B$ in rest-frame, ii) for the redshifted and "stretched" SED in observer's frame and iii) the SED type $T$. The individual correction factors $k_{B}$ have been derived via synthetic photometry, this topic is described in section 4.6.4.

$A_{B}^{i}$ is the correction of the intrinsic absorption due to dust within the distant galaxies. Two ba- sic approaches have been established for TF applications: One that only depends on the disk inclination (Tully \& Fouqé 1985) and one that additionally depends on the total mass (and hence the maximum rotation velocity). The latter was introduced by Tully et al. (1998). Both conventions will be described in section 4.6.5.

\subsubsection{Apparent Magnitudes}

Total apparent magnitudes were derived with the Source Extractor package. This program offers different algorithms for the photometry and a choice between circular or elliptical apertures. While fixed circular apertures are the best option for the derivation of galaxy colors (see chapter 6.4.2), it is mandatory to prevent flux losses in the computation of the absolute magnitudes. For this purpose, variable elliptical apertures yield the best results.

SExtractor offers three algorithms for the latter approach, called Mag_iso, Mag_best and Mag_auto. While tests showed that the latter two are consistent to within a few $0.01 \mathrm{mag}$ for the FDF spirals in all bandpasses, the Mag_iso routine systematically yielded underestimated brightnesses, in particular for faint detections. The Mag_auto routine is based on the "first moment" algorithm by Kron (1980) and designed to best reproduce the total magnitudes of extended sources. This algorithm has been used in the study described here, as for the complete FDF photometric catalogue (Heidt et al. 2003).

\subsubsection{Galactic Absorption}

For the Galactic absorption at the coordinates of the FDF, the values which are given in Heidt et al. (2003) were adopted. These were computed with the formulae given in Cardelli et al. (1989) under the assumption of $E(B-V)=0.018^{m}$ (derived from Burstein \& Heiles 1982) and $A_{V}=3.1 \cdot E(B-V)$.

For the individual broadband filters used in the FDF imaging, the resulting respective absorp- 
tion factors are $A_{U}^{g}=0.087^{m}, A_{B}^{g}=0.076^{m}$, $A_{g}^{g}=0.062^{m}, A_{R}^{g}=0.041^{m}$ and $A_{I}^{g}=0.035^{m}$.

\subsubsection{Distance Modulus}

The derivation of the luminosity distance and therewith the distance modulus in a universe with a non-zero cosmological constant is described, e.g., in Peebles (1993) and will not be outlined here. However, it is important to consider whether differences to the adopted cosmologies in previous studies could affect the comparison with literature data.

In almost all cases, a non $-\Lambda$ cosmology with $H_{0}=75 \mathrm{~km} \mathrm{~s}^{-1} \mathrm{Mpc}^{-1}$ has been assumed, either in an open universe with $\Omega_{m}=0.1$ (hereafter model B, e.g., Vogt et al. 1997, Vogt 2000, Milvang-Jensen 2003, Swinbank 2003) or a flat universe with $\Omega_{m}=1.0$ (hereafter model C, e.g., Rix et al. 1997, Simard \& Pritchet 1998). Besides the study described here, the concordance cosmology (model $\mathrm{A}$ in the following) has been adopted only in a TF project on 19 distant spirals presented by Barden et al. (2003). The distance moduli for the three different models are shown as a function of redshift in Fig. 4.17.

A fraction of the differences between model $\mathrm{A}$ on the one hand and B \& C on the other hand are of course due to the different values for $H_{0}$, these amount to $0.15 \mathrm{mag}$. The remaining, redshiftdependent offset between $\mathrm{A}$ and $\mathrm{B}$ is at maximum $\Delta D M=0.16^{m}$ in the regime $z \approx 0.6$, whereas the shift between $\mathrm{A}$ and $\mathrm{C}$ increases monotonically with look-back time and is $\Delta D M=0.60^{\mathrm{m}}$ at $z=1$. It should be noted, however, that the studies mentioned above which assumed model C focussed on spirals at moderate redshifts.

Explicit offsets in the distance moduli according to the covered redshifts are shown in Table 4.2. The given values of $\triangle D M$ have to be applied as correction factors prior to a comparison of the respective studies to the results of the FDF TF project, i.e., luminosties given in these previous studies have to be increased by $\triangle D M$.

Note that, if only luminosity offsets compared

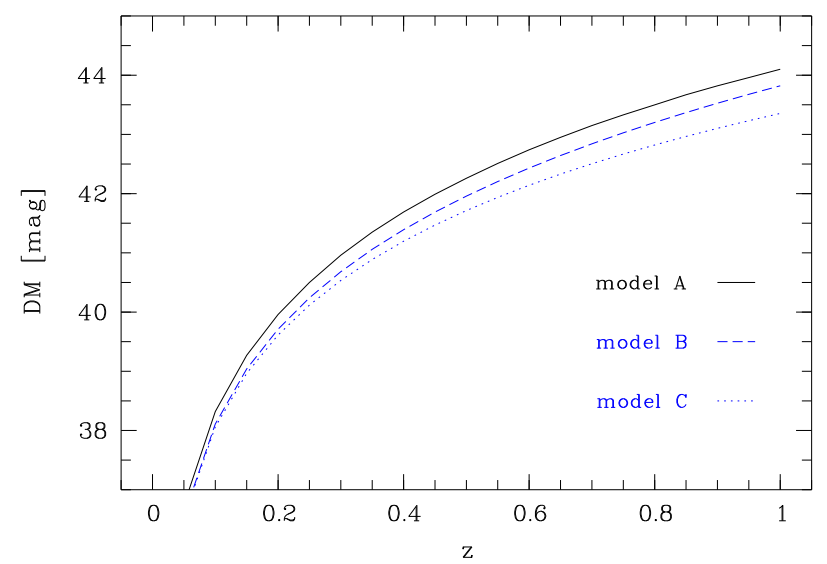

Figure 4.17: Distance moduli for three different cosmologies used in previous TF studies. Model A denotes the concordance cosmology which also is adopted here, the two other are models without a cosmological constant, see text for details.

to a given local TFR are considered, the correction factors have to be reduced by $0.15^{\mathrm{m}}$ since all the studies listed in Table 4.2 assumed $H_{0}=75 \mathrm{~km} \mathrm{~s}^{-1} \mathrm{Mpc}^{-1}$.

Table 4.2: Corrections that will have to be applied to the distance moduli in some previous studies to reach consistency with the concordance cosmology adopted here.

\begin{tabular}{lccl}
\hline Study & model & $\langle z\rangle$ & $\Delta D M$ \\
& & & \\
\hline Vogt et al. / Vogt & $\mathrm{B}$ & $\sim 0.5$ & $\sim 0.3^{m}$ \\
Rix et al. & $\mathrm{C}$ & $\sim 0.25$ & $\sim 0.4^{m}$ \\
Simard \& Pritchet & $\mathrm{C}$ & $\sim 0.35$ & $\sim 0.45^{m}$ \\
Milvang-Jensen & $\mathrm{B}$ & $\sim 0.4$ & $\sim 0.3^{m}$ \\
Swinbank et al. & $\mathrm{B}$ & $1.034^{\dagger}$ & $\sim 0.3^{m}$ \\
& & & \\
\hline${ }^{\dagger}$ this study was focussed on a single spiral \\
\hline
\end{tabular}

\subsubsection{K-Correction}

Now, the transformation from an observed apparent brightness in a passband $X$ to a corre- 
sponding rest-frame brightness in a passband $Y$ will be described. Such a transformation defines a $k$-correction of the form

$$
k_{Y}(X, T, z)=X(T, z)_{\mathrm{obs}}-Y(T, 0)_{\text {rest }}
$$

If available broad band information is limited to a few filters (two in most previous studies), the different wavelength ranges covered by a passband in observer's frame and rest-frame introduce a strong dependence of the achievable $k$ correction accuracy on SED type. At redshift $z=0.6$, e.g., the $k$-correction difference between the Sbc and Scd types corresponds to a change of half a magnitude in the transformation from $B_{\text {obs }}$ to $B_{\text {rest }}, k_{B}$ (e.g., Frei \& Gunn 1994). Values of $k_{B}$ differ even more for earlier types, thus even a slight misclassification can introduce a substantial offset in the derived luminosity if observations are limited to one or two filters. In contrast to this, the project described here could exploit the multi-band imaging of the FDF. With the photometry available in $B, g, R$ and $I$, the filter that best matched the rest-frame $B$-band could be used to transform an apparent magnitude $X$ into absolute magnitudes $M_{B}$. Even for the highest redshifts $(z \approx 1)$ in the FDF sample, $k$-corrections were small $\left(\left|k_{B}\right|<0.5^{m}\right)$.

To achieve the highest possible accuracy - note that the FORS filter system and the standard Johnson-Cousins system are slightly different the $k_{B}$-corrections were not taken from the literature but computed via synthetic photometry. For a given SED of type $T$ at redshift $z$ with wavelength-dependent flux $F(T, \lambda)$, the brightness in a passband $X$ was defined as

$$
\begin{aligned}
m_{X}(T, z)= & -2.5 \log \frac{\int F(T, z, \lambda) S_{X}(\lambda) d \lambda}{\int S_{X}(\lambda) d \lambda} \\
& +C_{X}+C_{\mathrm{sys}}
\end{aligned}
$$

(e.g., Fritze-v. Alvensleben 1989). Here, $S_{X}(\lambda)$ is the response function of filter $X, C_{X}$ is a constant for the calibration of this filter within the corresponding system (e.g, the Johnson or Cousins filter system) and $C_{\text {sys }}$ is an optional constant to calibrate between different filter systems. Note that Eq. 4.23 yields relative magnitudes which are appropriate for the transformation between different filters or different systems, but is not reliable for the computation of physical apparent brightnesses in the given form. For the latter purpose, and additional calibration constant would be needed, which could be derived via synthetic photometry with an observed SED of a galaxy with known apparent brightness. However, since the synthetic photometry only was performed to derive the $k$-corrections, i.e., relative magnitudes, this calibration was not needed.

In Fig. 4.18, the FORS filters $U, B, R, I$ and Gunn $g$ are shown; these were used for the imaging of the FDF in the optical regime. In Fig. 4.19, the filter respsonse curves of the standard Johnson-Cousins system $\left(U_{J}, B_{J}, V_{J}, R_{C}\right.$, $\left.I_{C}\right)$ are displayed; all curves were retrieved as ascii tables from the ESO webpages. These were used for the definition of the respective response functions $S_{X}$. In the next step, the calibration factors $C_{X}$ and $C_{\text {sys }}$ had to be derived.

For the Johnson system, the constants $C_{X}$ were taken from Allen (1973). They are defined in such a way to yield colors $(X-Y)=0.0$ for an A0V star spectrum in all passbands $X$ and $Y$. To calibrate the FORS filter system, the A0V spectrum from Pickles (1998) was used, the fine-tuning of the respective factors $C_{X}$ was performed with the equations given in Sinachopoulos \& van Dessel (1998). Finally, the constant $C_{\text {sys }}$ for transformations between the FORS and the Johnson system was calculated from the color $\left(V_{\text {fors }}-V_{J}\right)$ (ibid.). For the filters Cousins $R_{C}$ and $I_{C}$, the factors $C_{R}$ and $C_{I}$ from the FORS calibration were adopted as initial values (this approach was motivated by the very similar response curves of $R$ and $I$ in these two systems) and verified by a comparison of the colors $\left(R_{J}-R_{C}\right)$ and $\left(I_{J}-I_{C}\right)$, computed for various SED types, to the values given in Fukugita et al. (1995), yielding a consistency to within less than 


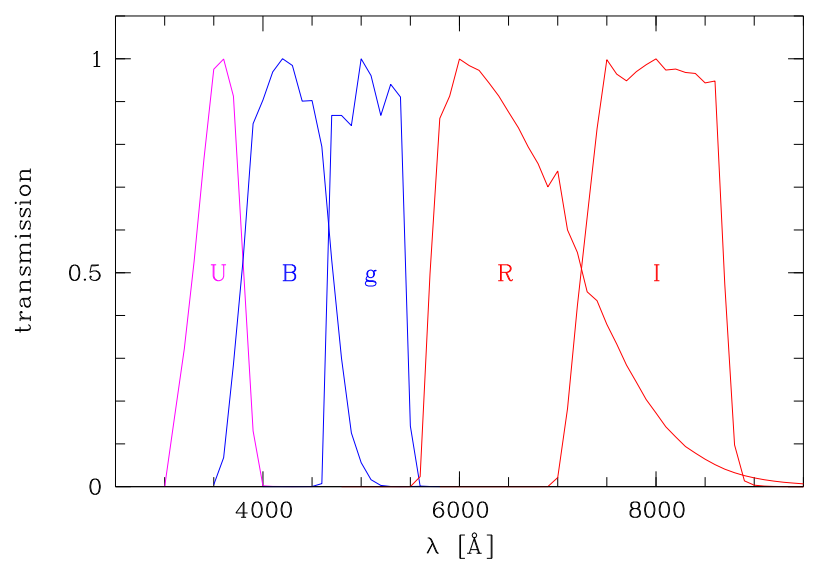

Figure 4.18: Transmission curves of the FORS $U$, $B, g, R$ and $I$ filters which were used for the imaging of the FDF.

0.03 mag.

A more complicated procedure was necessary for the calibration of the FORS $g$ filter, since it is slightly different from the original definition within the Thuan-Gunn filter system. More precisely, the FORS $g$ filter is broader by $\sim 90 \AA$ and has a central wavelength $\sim 130 \AA$ higher than the Thuan-Gunn $g$. A first calibration was performed using the transformation

$$
g_{\text {gunn }}-V_{J}=0.503\left(B_{J}-V_{J}\right)-0.226
$$

from Jørgensen (1994). Based on the A0V spectrum, the color $\left(g_{\text {fors }}-g_{\text {gunn }}\right)$ then was computed, yielding a value of $0.10 \mathrm{mag}$ and thereby a difference to the Johnson System of $\left(g_{\text {fors }}(A 0 V)-V_{J}(A 0 V)\right)=-0.13^{m}$. This was cross-checked by a comparison to colors based on various definitions of the $g$ passband given in Fukugita et al.

As SED templates for the computation of the $k$ corrections, the spectra published by Möller et al. (2001) were used. These templates were generated with evolutionary synthesis models. The authors computed two versions of each template, one including dust reddening and one without dust. For the synthetic photometry described here, templates without dust were used solely, since the intrinsic absorption was accounted for

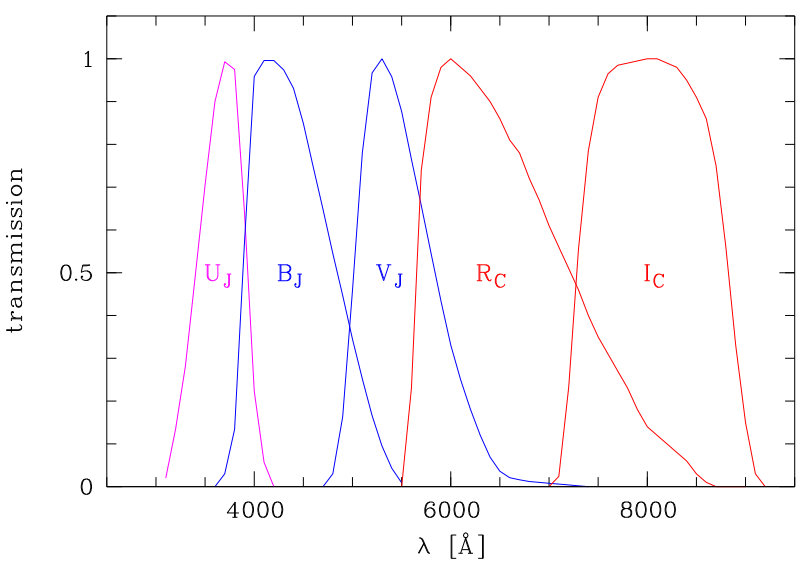

Figure 4.19: Transmission curves of the Johnson $U_{J}, B_{J}, V_{J}$ and Cousins $R_{C}, I_{C}$ filters.

in a separate computation step (see next section). The basic advantage of synthetic spectra with respect to observed spectra is of course that no noise is introduced in the photometry.

Five different types were used for the derivation of the $k$-corrections: Sa (age 11 Gyrs), Sb (11 Gyrs), Sc (11 Gyrs), Sdm (9 Gyrs) and Im (3 Gyrs). The respective ages were chosen to reproduce the observed colors of present-day galaxies (see further below). The templates are shown in Fig. 4.20. For the purpose of this graph, all spectra have been normalized to the flux at $5500 \AA$. Gas emission was not incorporated in the models, but this has a negligible effect on broad band photometry. Even for an extremely latetype SED with an emission line equivalent width of $100 \AA$, observed with the relatively narrow $g$ filter, the contribution of the emission line to the total measured flux would be only $0.13^{\mathrm{m}}$.

The SEDs were redshifted by calculating the flux $F$ at wavelength $\lambda$ according to

$$
F(T, z, \lambda)=\frac{F_{0}(T, \lambda /(1+z))}{(1+z)}
$$

(e.g., Contardo, Steinmetz \& Fritze-v. Alvensleben 1998), where $F_{0}$ is the flux of the unredshifted spectrum. Finally, the transformation from the apparent magnitude of a spectrum of type $T$ at redshift $z$ observed with a FORS fil- 

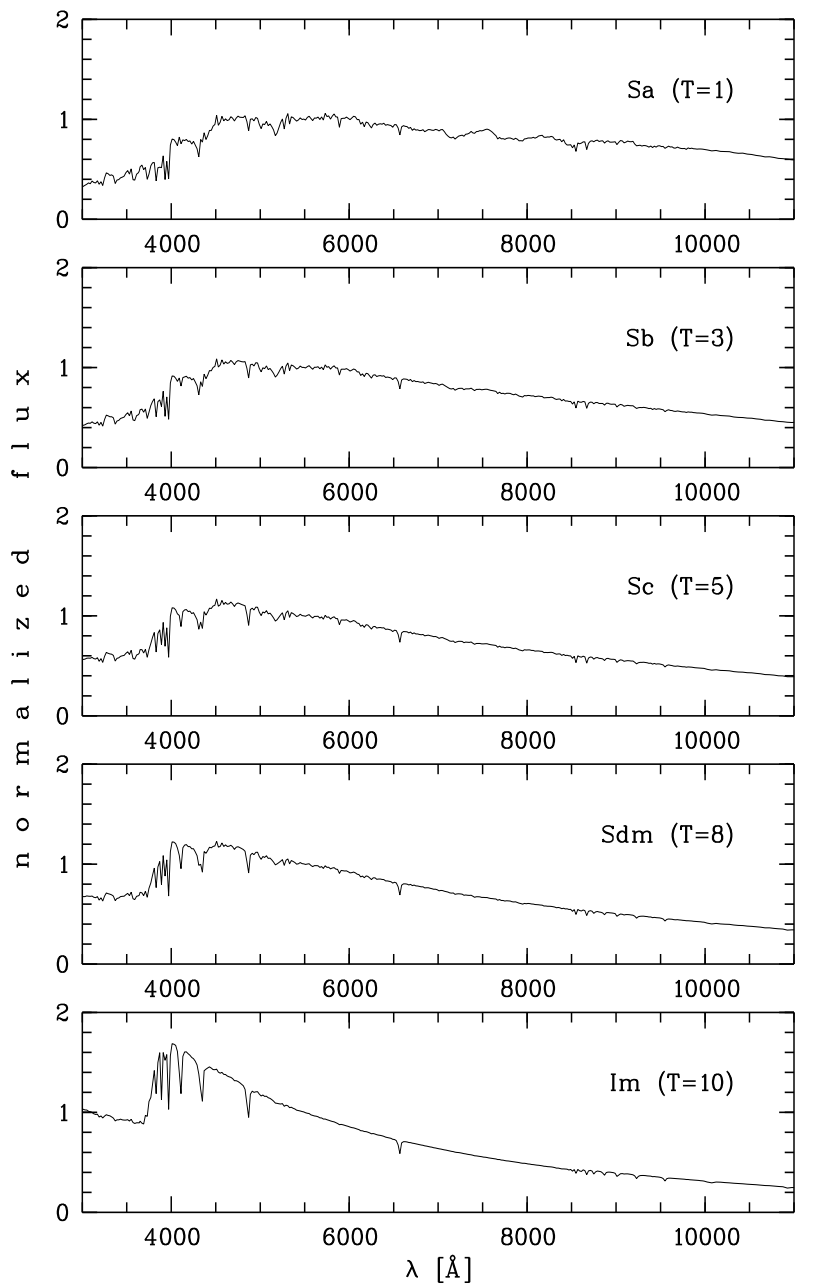

Figure 4.20: SED templates used for the computation of the $k$-corrections via synthetic photometry. These spectra were generated via chemically consistent evolutionary synthesis models (Möller et al. 2001). For this plot, the templates have been normalized to their flux at $5500 \AA$.

ter $X$ to the Johnson $B$ magnitude in rest-frame was performed via

$$
k_{B}(X, T, z)=m_{X}(T, z)-m_{B}(T, z=0),
$$

where the two terms on the right hand side were derived according to Eq. 4.23. $K$-corrections were computed with the equation given above for all source filters, types and redshifts covered by the FDF spirals.

Respective filters used for the apparent magni- tudes in Eq. 4.21 were $B_{\text {fors }}$ for $z<0.25, g_{\text {fors }}$ for $0.25 \leq z<0.55, R_{\text {fors }}$ for $0.55 \leq z<0.85$ and $I_{\text {fors }}$ for $z \geq 0.85$. This way, the $k$-correction was much less sensitive to spectral type than a transformation $B_{\text {obs }} \rightarrow B_{\text {rest }}$, especially at higher redshifts: e.g., $k_{B}(R, 1,0.6)-k_{B}(R, 5,0.6)=0.05^{m}$ whereas $k_{B}(B, 1,0.6)-k_{B}(B, 5,0.6)=0.64^{m}$.

For testing purposes, the colors of the templates were derived purely within the Johnson-Cousins Filter system and compared to the values published by Fukugita et al. The deviations were very small, their absolute values fell into the range $0.03^{m} \leq|\Delta(X-Y)| \leq 0.08^{m}$.

\subsubsection{Intrinsic Absorption}

The last critical correction that has to be applied to the observed magnitudes is the intrinsic restframe $B$ absorption $A_{B}^{i}$ due to the dust disks of the spirals. Since the amount of absorption depends on the optical depth along the line-ofsight, it is a function of the disk inclination $i$. Basically, two conventions have been established in the literature.

The older one of these is the approach by Tully \& Fouqué (1985, TF85 hereafter). In the previous studies on distant spirals, this method has been used solely. For the sake of comparability, it was therefore also considered the first option for the analysis of the FDF spirals.

Based on geometric assumptions, TF85 derived the formula

$$
A_{B}^{i}=-2.5 \log \left[f\left(1+e^{-\alpha}\right)+(1-2 f)\left(\frac{1-e^{-\alpha}}{\alpha}\right)\right]
$$

with $\alpha=\tau \sec i$. Here, $f$ is the fractional dust disk thickness on either side of the disk which can range between 0.5 (corresponding to an infinitesimal thin dust disk) and 0 (same scale height of dust disk and luminous disk). $\tau$ is the optical depth.

With a sample of 101 local spirals, TF85 derived best-fitting values of $\tau=0.55$ and $f=0.25$, i.e. the dust disk has half the scale height of the luminous disk. These values were also adopted 
here and held fixed, hence the TF85 absorption is purely inclination-dependent. The authors pointed out that their approach is reliable only for disks up to $i=80^{\circ}$, whereas above this value, unobscured light from the "back side" of the disk would decrease the effective amount of absorption. However, even the most edge-on FDF spiral is within the given limit and Eq. 4.27 therefore could be used for the entire sample.

For the TF objects, $A_{B}^{i}$ spans values from $0.30^{m}$ at $i=25^{\circ}$ to $0.96^{m}$ at $i=80^{\circ}$. Note that the absorption for face-on disks is finite in this convention: $A_{B}^{i}=0.27^{m}$ for $i=0^{\circ}$. The multi-band photometry allows to compute the absorption for the same rest-frame wavelength intervall at all the covered redshifts.

More recently, Tully et al. (1998, Tu98 hereafter) and Karachentsev et al. (2002) have found evidence for an internal extinction law which not only depends on the inclination but also on the maximum rotation velocity (and therefore hints to a dependency on the total mass). These observations favour a higher amount of absorption in fast rotators than for spirals of low $V_{\max }$. For the rest-frame $B$-band, Tu98 found a correlation between intrinsic absorption $A_{B}^{i, W}$, inclination $i$ and H I profile linewidth $W$ which is given by

$$
A_{B}^{i, W}=(1.57+2.75(\log W-2.5)) \log (a / b)
$$

If one assumes the relation $2 V_{\max } \approx W$ (see TF85), then equation 4.28 transforms into

$$
A_{B}^{i, V}=2.75\left(\log V_{\max }-1.63\right) \log (a / b),
$$

where $a$ and $b$ are the apparent major and minor axis, respectively. E.g., for a highly inclined disk with $i=80^{\circ}$, this yields $0.59^{m}$ at $V_{\max }=100 \mathrm{~km} / \mathrm{s}$ and $1.35^{m}$ at $V_{\max }=300 \mathrm{~km} / \mathrm{s}$, whereas the initial TF85 approach gives a value of $A_{B}^{i}=0.96^{m}$ independent of the maximum rotation velocity.

A more detailed comparison between the two methods is shown in Fig. 4.21. No values for $i>80^{\circ}$ are displayed to account for the usable range of the TF85 approach. Three differ-

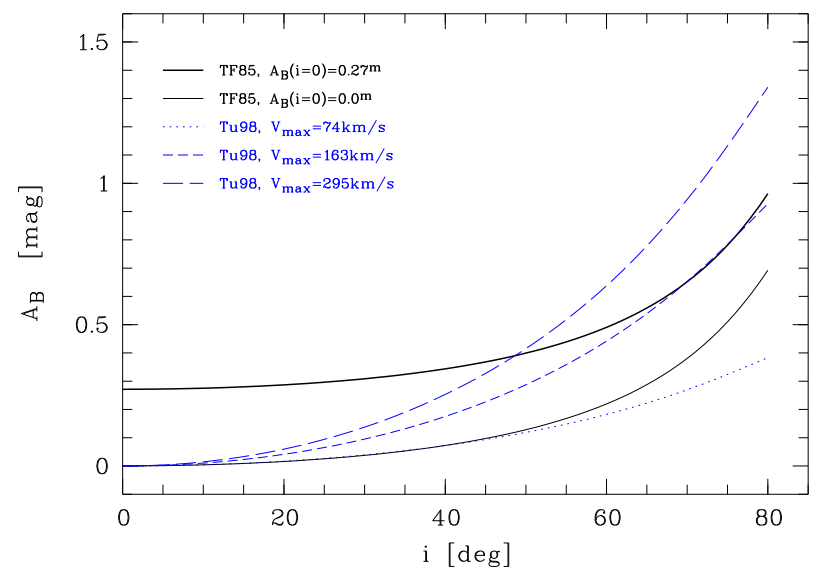

Figure 4.21: Comparison of the two most established conventions for intrinsic absorption correction: Tully \& Fouqé (1985, TF85) and Tully et al. (1998, Tu98). The thick and thin solid lines denote the TF85 approach with non-negligible and negligible face-on extinction, respectively. The dotted, short-dashed and long-dashed lines give the massdependent Tu98 convention for maximum rotation velocities of $74 \mathrm{~km} / \mathrm{s}, 163 \mathrm{~km} / \mathrm{s}$ and $295 \mathrm{~km} / \mathrm{s}$, respectively. All displayed extinction functions correspond to rest-frame $B$.

ent values of $V_{\max }$ are shown for the Tu98 convention which represent the minimum $(74 \mathrm{~km} / \mathrm{s})$, median $(163 \mathrm{~km} / \mathrm{s})$ and maximum $(295 \mathrm{~km} / \mathrm{s})$ of the TF sample distribution, restricted to 36 rotation curves which reach well into the region of constant velocity at large radii.

For the usage of Eq. 4.29, axial ratios $a / b$ corresponding to given inclinations have been computed via

$$
a / b=\left(0.96 \cos ^{2} i+0.04\right)^{-1 / 2}
$$

(see Tu98). Two variants of the TF85 approach are shown in Fig.4.21. In the first one (denoted by the thick solid line), even for a face-on disk (i.e., $i=0^{\circ}$ ) the intrinsic rest-frame $B$ absorption is $0.27 \mathrm{mag}$. This non-negligible extinction is simply due to the finite height of the dust disk. To compare it to the Tu98 approach, the TF85 convention was alternatively computed as

$$
A_{B}^{i, 2}=A_{B}^{i}-0.27
$$


(denoted by the thin solid line in Fig.4.21). Note that for the comparison between a local and a distant $\mathrm{TF}$ sample, it does not matter which amount of face-on absorption is assumed as long as both samples are corrected for absorption consistently. However, since a zero face-on extinction basically is physical only for an infinitely thin dust disk, the convention of $A_{B}^{i}(i=0)=0.27^{m}$ has been used as the first option in this thesis.

Only for relatively small spirals with $V_{\max } \approx 100 \mathrm{~km} / \mathrm{s}$, Eqs. 4.29 and 4.31 give consistent estimations of the intrinsic absorption. For more massive galaxies, the Tu98 approach yields much larger values. Moreover, at a given inclination, the absorption according to Tu98 is larger for higher $V_{\max }$. In effect, the slope of any TFR (parameterised as $\left.M \propto a \log V_{\max }\right)$ is steeper if the intrinsic absorption is accounted for based on Eq. 4.29. Whether the results of the TF analysis of the FDF spirals would be affected by this will be discussed in chapter 6.4.6.

\subsection{Luminosity Distribution and Errors}

The distribution of the TF spirals in absolute $B$ band magnitude is shown in Fig. 4.22. A range of $-22.63 \leq M_{B} \leq-17.88\left(\left\langle M_{B}\right\rangle=-20.40\right)$ is covered by the $77 \mathrm{TF}$ spirals, while the sub-sample with high quality rotation curves, i.e. curves which reach well into the region of constant rotation velocity, shows a spread $-22.25 \leq M_{B} \leq-18.58\left(\left\langle M_{B}\right\rangle=-20.79\right)$. A set of 36 spirals with determined redshifts which were not usable for the $V_{\max }$ derivation cover a range $-22.45 \leq M_{B} \leq-14.78$ $\left(\left\langle M_{B}\right\rangle=-19.89\right)$. Note that $0.20^{m}$ of the $0.51^{m}$ difference in the median absolute magnitude with respect to the TF objects can be attributed to a slightly lower median redshift of the non-TF spirals. A similar factor of $0.21^{m}$ contributes to the difference in $\left\langle M_{B}\right\rangle$ between

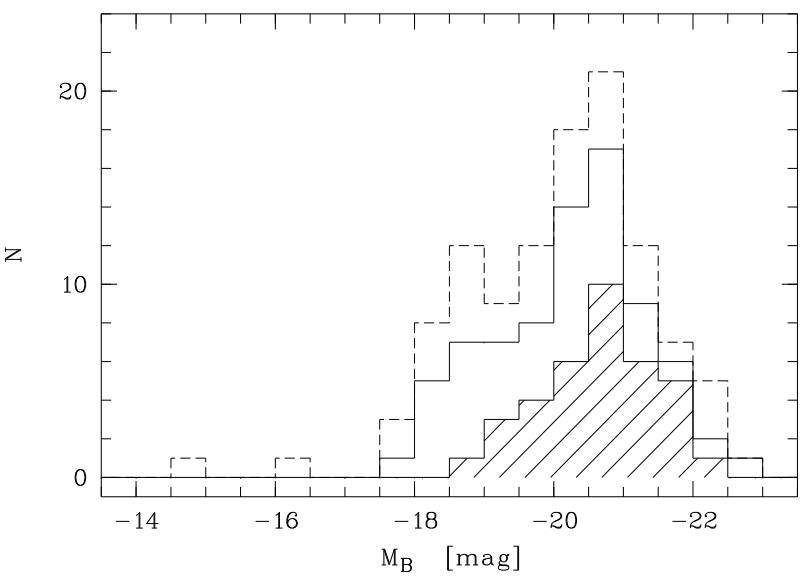

Figure 4.22: Absolute $B$-band magnitude distribution of all 113 FDF spirals with determined redshifts (dashed line), the sub-sample of $77 \mathrm{TF}$ objects which could be used for the derivation of $V_{\max }$ (solid line) and the subset of $36 \mathrm{TF}$ spirals with high quality rotation curves (shaded region).

the high quality and low quality data.

In Fig. 4.23, the absolute magnitudes are shown as a function of redshift. Similar to Fig. 4.22, all 113 spirals with determined redshifts are included, splitted into spirals with or without a value for $V_{\max }$. The former subset in turn is subdivided according to the rotation curve quality. Note that one faint spiral with $M_{B}=-16.15$ at $z=0.0338$ did yield a value for $V_{\max }$ but was rejected from the TF sample due to its very low redshift. According to an upper limit on the systematic velocity of $V_{\text {sys }}<15000 \mathrm{~km} / \mathrm{s}$ adopted here and also in other works (e.g., Haynes et al. 1999), this spiral was considered a local object. Additionally to the individual objects, the limits in absolute magnitude corresponding to the selection criterion $R \leq 23^{m}$ are displayed in Fig. 4.22. These functions $M_{B}=f(z)$ were computed via Eq. 4.21 with a $k$-correction $R_{\text {fors }} \rightarrow B_{J}$ for SED types Sa, Sc and Im. To estimate the typical intrinsic absorption in restframe according to TF85, the median inclination $i=53^{\circ}$ of the $\mathrm{TF}$ sample was used, and the absorption coefficients with respect to the $B$-band were derived with the equations given in Cardelli 


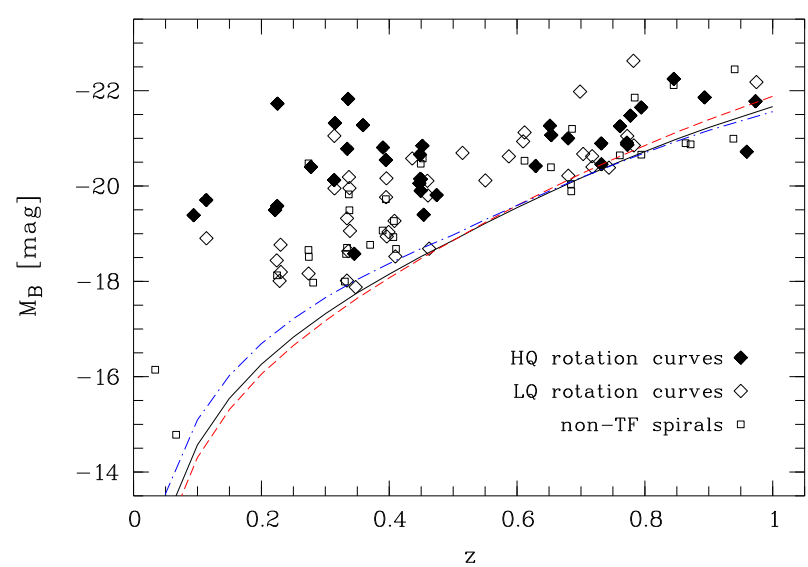

Figure 4.23: Absolute B-band magnitudes of all 113 FDF spirals with derived redshifts, plotted against $z$. The dashed, solid and dot-dashed lines show the absolute magnitudes corresponding to $R=23^{m}$ for SED types $T=1,5$ and 10 , respectively. A few objects with $R>23^{m}$ exceeed the limits.

et al. (1989).

A couple of conclusions can be made on the basis of Fig. 4.23. Firstly, though only a single filter, the FORS $R$-band, has been used for the transformation of the brightness constraint into $M_{B}$ values, the functions denoting the limits are in good agreement with the "faint end" of the observed distribution. Only a few objects with $R>23^{m}$ exceed the given limits, these are spirals which had to be selected for spectroscopy with a slightly relaxed brightness constraint.

Secondly, since the magnitude limitation is only weakly type-dependent, it is unlikely that our selection on apparent $R$-band brightness could have introduced a bias towards certain types. This conclusion is strengthened by the homogeneous redshift distribution of the sub-samples (see section 4.2).

And thirdly, Fig. 4.23 indicates that the sample is volume-limited towards the high-luminosity end of the $M_{B}$-distribution. One may tentatively derive a high-luminosity "cutoff" in the regime $M_{B} \approx-22.5^{m}$ with a slight depependence on redshift. Either the luminosity function is truncated in this magnitude regime or, more propably, it is very steep. In turn, the number density of extremely luminous spirals could be too low for the FDF volume to contain objects of corresponding absolute magnitude. To verify this quantitatively, the volume probed with the FDF (assuming an effective field--of-view of $36 \operatorname{arcmin}^{2}$ ) was computed following Carroll \& Press (1992). At, e.g., $z=0.3$ and $z=1$, the respective volumes are $2.4 \cdot 10^{3} \mathrm{Mpc}^{3}$ and $1.4 \cdot 10^{4} \mathrm{Mpc}^{3}$. If one adopts the local $B$-band luminosity function (LF) as given in Fried et al. (2001), the expected number of spirals drops below 1 for $M_{B}<-21.3$ at $z=0.3$ and for $M_{B}<-22.0$ at $z=1.0$. Note, however, that this approach is just a simple estimate. Moreover, an evolution of the LF was neglected here. Fried et al., e.g., find an increase of the luminosity density by a factor of 3.5 up $z=1$ for types Sa to Sc. Nevertheless, the limitation in volume is the most probable explanation for the highluminosity cutoff in the FDF spiral distribution. The errors on all absolute magnitudes were computed via

$$
\sigma_{M B}^{2}=\sigma_{X}^{2}+\sigma_{k}^{2}+\sigma_{A B}^{2} .
$$

Here, $\sigma_{X}$ is the photometric error in the respective filter. Thanks to the very deep imaging of the FDF, these uncertainties are very small. For the $77 \mathrm{TF}$ spirals, the median values are $\left\langle\sigma_{X}\right\rangle<0.015^{m}$ in $B, g, R$ and $I$, respectively. $\sigma_{k}$ denotes the $k$-correction error. If one assumes an SED type uncertainty of $\Delta T=2$ (i.e., an Sc spiral could be misclassified as an $\mathrm{Sb}$ and vice versa), the corresponding error of the $k$ correction is $\sigma_{k} \leq 0.1^{m}$ for all types and redshifts covered by the FDF spirals.

Finally, $\sigma_{A B}$ in Eq. 4.32 gives the uncertainty of the intrinsic absorption correction via error propagation from the inclination error.

Contributions of the distance modulus or the galacitc absorption to $\sigma_{M B}$ were assumed to be negligible. For the TF sample, the total error falls into the range $0.07^{m} \leq \sigma_{M B} \leq 0.21^{m}$ with a median of only 0.094 mag. 


\section{Chapter 5}

\section{Kinematic Analysis}

An important characteristic in favour of the FDF spiral data is the spatially resolved spectroscopy. If one cannot derive the disk rotation velocity as a function of radius but is restricted to measurements of the emission line widths $W_{c}$ (broadend due to the approaching and departing sides of the rotating disk), then it is mandatory to take into account the contributions from the velocity dispersion in the disk:

$$
W_{c}=\left[\left(\frac{W_{\mathrm{obs}}-\Delta_{\mathrm{ins}}}{1+z}\right)^{2}-\Delta_{t}^{2}\right]^{1 / 2} \frac{1}{\sin i}
$$

(e.g., Giovanelli et al. 1997), where $W_{\text {obs }}$ is the observed linewidth, $\Delta_{\text {ins }}$ is the correction factor for instrumental broadening and $\Delta_{t}$ accounts for the broadening due to the random motions in the disk. However, the value of $\Delta_{t}$ is unknown in purely linewidth-based measurements. This systematically increases the observed scatter of $W_{c}$ (by $\sim 20 \%$ in the case of the Rix et al. 1997 sample).

Moreover, the spatial region from which the emission originates cannot be derived from linewidths measurements. [O II] doublet emission, e.g., has been observed to be frequently concentrated towards small galactocentric radii of spiral galaxies (Bershady 1997). Since this potentially involves a significant light contribution from the bulge component (which in contrast to the disk is stabilized by random motions), the systematic error on $W_{c}$ is further increased. All these problems do not affect the FDF spiral data thanks to the spatially resolved rotation curves
(RCs).

For distant, apparently small spirals, the measurement of rotation velocities as a function of radius, $V_{\text {rot }}^{\mathrm{o}}(r)$, is a much more complicated topic than for "local" objects of redshifts below $\sim 0.03$. This has basically two reasons:

Firstly, the extent of the seeing disk ( $\sim 1$ arcsec) is comparable in size to the optical disk of the spiral, whereas local spirals typically extend over one arcminute. In effect, the observed velocity fields of distant spirals are heavily smoothed with respect to their low $-z$ counterparts.

And secondly, the effect of the slit width must be taken into account. At redshift $z=0.5$, e.g., a scale length of $3 \mathrm{kpc}$ - typical for an $L^{*}$ spiral - corresponds to $\sim 0.5$ arcsec only, which is half the slit width used in the MOS observations of the FDF spirals. Any value of $V_{\text {rot }}^{\mathrm{o}}(r)$ is therefore an integration of the velocity field perpendicular to the spatial axis (slit direction), a phenomenon which is the optical equivalent to "beam smearing" in radio observations. If not taken into account, this effect could lead to an underestimation of the intrinsic rotation velocities, in particular the intrinsic values of $V_{\max }$ which are needed to construct the TFR.

One way to overcome this problem is to perform the simulation of an RC taking into account all observational effects, and compare it to the observed one. For a given spiral, the best reproduction of an observed curve by a simulated curve (of which all parameters are known by definition) can be used to derive the intrinsic maximum ro- 
tation velocity.

In the next section, the measurements of the observed RCs will be described, followed by the RC modelling procedure in section 5.2. Different approaches of $\mathrm{RC}$ shapes will be compared. The distribution in $V_{\max }$ of the FDF spirals is discussed in section 5.3. The last section describes the derivation of the total masses.

A data table of the results presented in this chapter can be found in appendix B.

\subsection{Rotation Curve Extraction}

The measurements of the rotation velocity as a function of radius were performed in a semi-automatic manner with a special program rotcurve.

In the first step, the one-dimensional spectrum was visually inspected for potentially usable emission lines. Then, 100 columns of the twodimensional spectrum centered on the considered emission line were averaged to get a profile of line plus continuum along the spatial axis. This profile was approximated with a Gaussian to derive the optical center to within 0.1 arcsec. For a few objects located very close to the slit edge, the center was redefined manually.

Following this, automatic row-by-row fits were applied to the emission line. To enhance the $\mathrm{S} / \mathrm{N}$, three neighbouring rows were averaged prior to the emission line fitting, i.e., for a fit to row $n$, the rows $n-1$ to $n+1$ were averaged. In the case of very weak emission lines, this so-called boxcar filter was set to five rows, corresponding to one arcsecond. The fitting itself was performed with the MIDAS-algorithm DEBLEND/LINE which applied a least-square fit of a Gaussian profile to the continuum-subtracted, boxcar-filtered row $n$ of the spectrum. Central flux, $\sigma$ and central wavelength were free parameters in these fits.

A single Gaussian was used for [O III] 4959, [O III] 5007, $\mathrm{H} \beta$ or $\mathrm{H} \alpha$ (with the latter being visible only in four spectra), whereas two Gaussians of equal FWHM and an observer's frame separation of $2.75(1+z) \AA$ were used in the case of the $[\mathrm{OII}]$ doublet. For spectra of low $\mathrm{S} / \mathrm{N}$, however, fits of a single profile to the [O II] line yielded better results in terms of smaller errors and larger radial extent of the extracted RC.

If one computes the apparent galactocentric radius $r$ from the respective row numbers $n$ in the 2-D spectrum via

$$
r=\left(n_{\mathrm{c}}-n\right) s,
$$

where $n_{\mathrm{c}}$ denotes the row corresponding to the optical center and $s$ is the spatial scale of $0.2 \mathrm{arcsec} / \mathrm{pix}$, then the red- and blueshifts along the spectral axis due to disk rotation can be transformed into line-of-sight rotation velocities according to

$$
V_{\mathrm{rot}}^{\mathrm{o}}(r)=\frac{\left[(1+z) \lambda_{\mathrm{c}}-\lambda_{\mathrm{fit}}(r)\right] c}{(1+z) \lambda_{\mathrm{c}}}
$$

Here, $\lambda_{c}$ is the rest-frame wavelength of the emission line at the galaxies' optical center, $\lambda_{\text {fit }}(r)$ is the best-fitting wavelength as a function of apparent galactocentric radius and $c$ is the speed of light. Eq. 5.3 defines an observed rotation curve.

To derive the errors on $V_{\text {rot }}^{\mathrm{o}}(r)$, the uncertainties $\sigma_{\lambda}(r)$ of the respective best-fitting values $\lambda_{\text {fit }}(r)$ (yielded by DEBLEND/LINE) were transformed into rotation velocity errors $\sigma_{v}(r)$ by calculating

$$
\sigma_{v}(r)=\frac{\sigma_{\lambda}(r) c}{(1+z) \lambda_{\mathrm{fit}}(r)}
$$

At very low $\mathrm{S} / \mathrm{N}$, the Gaussian fits could be affected by residuals from the night sky subtraction or pixel defects. At large projected galactocentric radii, false detections may thereby affect the RC measurements. To minimize such effects a priori, the rotcurve algorithm rejected fits with a FWHM below $2 \AA$ (corresponding to half the instrumental resolution) or above $12 \AA$. 


\subsection{Simulated Rotation Curves}

The RC simulation developed for the FDF spirals (implemented in a special MIDAS program called rcmodel) is similiar to the procedure described by Simard \& Pritchet (1999, SP99 hereafter). However, there are some basic differences:

- While SP99 keep the disk scale length a free parameter, this is a fixed value in rcmodel which is derived from the broad band scale length. Moreover, the systematic deviations between continuum scale length and emitting gas scale length are taken into account (see next section for details).

- rcmodel accounts for the misalignment angle $\delta$ between slit and apparent major axis. In contrast to this, the SP99 approach assumes $\delta=0^{\circ}$, which is reliable only if the slits can be individually aligned with the apparent major axes during target selection (i.e., only for MXU data).

- In the SP99 approach, 2-D spectra are simulated and fitted to the observed 2-D spectra with the so-called Metropolis algorithm (e.g., Saha \& Williams 1994). In particular, no observed 1-D RCs are extracted. In contrast to this method, here the observed and simulated $R C s$ are compared.

- Since the shape of the observed RCs is not considered in the SP99 approach, the robustness of $V_{\max }$ is estimated with the normalized $\chi^{2}$ of the fit and the errors given by the Metropolis algorithm. Some shortcomings of such an "RC-blind" method will be addressed in section 5.2.2. In the case of the FDF spirals, the reliability of the derived $V_{\max }$ values is tightly linked to the quality of the $\mathrm{RC}$ as an additional criterion.

\subsubsection{The Intrinsic Shape}

In the first step, one has to assume an intrinsic rotational law $V_{\text {rot }}^{\mathrm{i}}(r)$. Three different kinds of

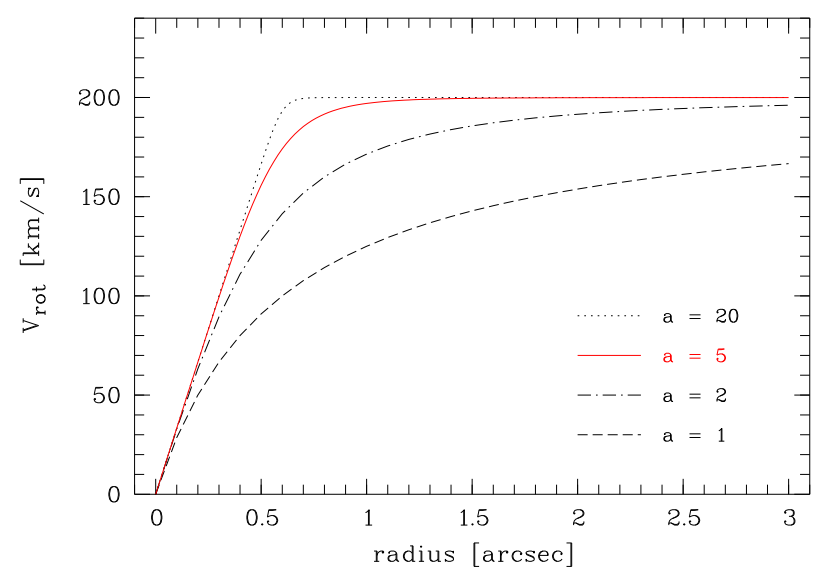

Figure 5.1: Templates of intrinsic rotation curves with a "rise-turnover-flat" shape. Different lines denote variations of the parameter $a$ in Eq. 5.5, the value $a=5$ was held fixed for all FDF spirals.

such an intrinsic RC were tested here. As the first and simplest approach, a parameterisation was used which features a linear rise of $V_{\text {rot }}^{\mathrm{i}}$ at small radii that turns over into a region of constant rotation velocity. This "flat" RC shape for large galactocentric radii corresponds to a mass distribution dominated by the Dark Matter halo. Hereafter, this "rise-turnover-flat" shape will be referred to as the "RTF shape". It has been used in most previous studies which could rely on spatially resolved RCs (e.g., Vogt et al. 1996, 1997, Barden et al. 2003).

The RTF shape of the intrinsic RC was computed via

$$
V_{\text {rot }}^{\mathrm{i}}(r)=\frac{V_{\max } r}{\left(r^{a}+r_{0}^{a}\right)^{1 / a}}
$$

(e.g., Courteau 1997) with a factor a that tunes the sharpness of the "turnover" at radius $r=r_{0}$, and a constant rotation $V_{\text {rot }}^{\mathrm{i}}(r)=V_{\max }$ for $r \gg r_{0}$. A range of examples for an apparent turnover radius $r_{0}=0.6$ arcsec is shown in Fig. 5.1. After tests with $\sim 20 \mathrm{RCs}$ from the FDF sample, a value of $a=5$ was kept fixed for all objects since it yielded the best results.

The turnover radius $r_{0}$ was assumed to be equal to the scale length of the emitting gas, which 
is larger than the scale length $r_{\mathrm{d}}$ derived from continuum emission. This is discussed in Ryder \& Dopita (1994) and Dopita \& Ryder (1994) using observational and theoretical approaches, respectively. Since the structural parameters like the continuum scale lengths were measured in the $I$ reference frame, the corresponding gas scale lengths were derived via

$$
r_{0}=(2-z / 2) r_{\mathrm{d}}
$$

This equation yields $r_{0} \approx 2 r_{\mathrm{d}}$ for the nearest FDF spirals (for which $I$ corresponds to restframe $R$ ) and $r_{0}=1.5 r_{\mathrm{d}}$ at $z=1$, where $I$ corresponds to rest-frame $B$. In other words, Eq. 5.6 is used to gain a correlation between restframe scale length and gas scale length that is in compliance with the results of Ryder \& Dopita. Eq. 5.6 was verified with a few high $\mathrm{S} / \mathrm{N}$ emission line profiles from the FDF spiral spectra. It should be noted however, that the $V_{\max }$ derivation is much less sensitive to the scale length than to the inclination and the misalignment angle. This is mainly due to the smoothing by the instrumental PSF.

As an alternative to the RTF shape, two other templates of intrinsic curves were considered. These were developed within the Universal Rotation Curve (URC) framework by Persic \& Salucci (1991, cited as PS91 hereafter, the parameterisation of the URC introduced therein will be referred to as URC91) and Persic et al. (1996, Pe96 and URC96 hereafter, respectively). While the URC91 definition has been used also by MilvangJensen et al. (2003), the URC96 has not been used by any previous distant TFR study, in spite of its more "realistic" shape (at small radii in particular) with respect to the URC91.

The URC approach introduces a dependence of the RC morphology on luminosity: Only spirals of luminosity $\sim L^{*}$ have a constant $V_{\text {rot }}$ at large radii, whereas the curves of sub- $L^{*}$ galaxies are rising even beyond a characteristic radius, and very luminous objects have a negative gradient at large radii.

$$
\begin{aligned}
V_{\text {rot }}^{\mathrm{i}}(r)= & V_{2.2}\left[1+\left(0.12-0.24 \log \frac{L_{B}}{L_{B}^{*}}\right)\right. \\
& \left.\times\left(\frac{r}{2.2 r_{\mathrm{d}}}-1\right)\right]
\end{aligned}
$$

Here, $V_{2.2}$ is the rotation velocity at $r=2.2 r_{\mathrm{d}}$. PS91 assumed an $L^{*}$-luminosity corresponding to $M_{B}^{*}=-21.2^{m}$, but since the authors used a Hubble constant of $H_{0}=50 \mathrm{~km} \mathrm{~s}^{-1} \mathrm{Mpc}^{-1}$, a value of $M_{B}^{*}=-20.5^{m}$ was adopted here. Note that the original URC91 definition did not include the parameter $V_{2.2}$ on the right hand side of Eq. 5.7, but

$$
V_{2.2}=200\left(\frac{L_{B}}{L_{B}^{*}}\right) \mathrm{km} / \mathrm{s}
$$

instead. In this form, however, it would not have been possible to derive $V_{2.2}$ as a free parameter. The URC91 shape is shown for three values of $M_{B}$ in Fig. 5.2. Note that in its original form, this RC was only defined for radii $r>r_{\mathrm{d}}$. For the sake of comparability to the observed RCs, a simple linear rise was therefore assumed in the range $0 \leq r \leq r_{\mathrm{d}}$.

The URC96 has the form

$$
\begin{array}{r}
V_{\text {rot }}^{\mathrm{i}}(x)=V_{\text {opt }}[(0.72+0.44 \log y) \\
\left.\times \frac{1.97 x^{1.22}}{\left(x^{2}+0.78^{2}\right)^{1.43}}+\frac{1.6 x^{2} \mathrm{e}^{-0.4 y}}{x^{2}+1.5^{2} y^{0.4}}\right]^{1 / 2}
\end{array}
$$

Here, $x=r / r_{\text {opt }}$ is the radius in units of the optical radius $r_{\mathrm{opt}}=3.2 r_{\mathrm{d}}$ (defined such that $83 \%$ of the total integrated light originates from $r \leq r_{\text {opt }}$ ) and $y=L_{B} / L_{B}^{*}$. With a Hubble constant $H_{0}=70 \mathrm{~km} \mathrm{~s}^{-1} \mathrm{Mpc}^{-1}$, the adopted value of $L^{*}$ corresponds to $M_{B}^{*}=-20.65^{m}$. The URC96 shape is shown in Fig. 5.3.

The characteristic radii which define a characteristic rotation velocity that can be used for the TF analysis differ slightly between the two approaches. For the URC91, this radius is equal to 2.2 disk scale lengths, whereas it is as large 


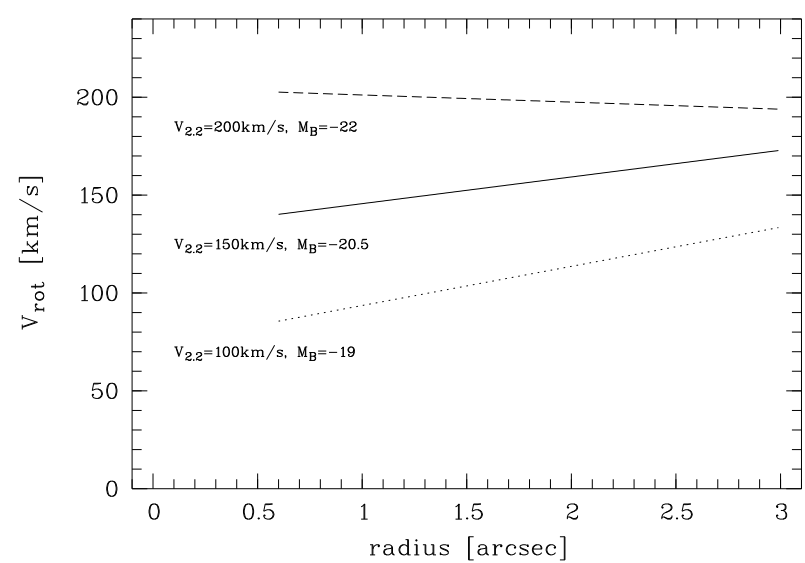

Figure 5.2: The Universal Rotation Curve (URC91) shape as introduced by Persic \& Salucci (1991). A gas scale length of 0.6 arcsec has been assumed. The velocity gradient depends on the absolute magnitude. For clarity, different characteristic rotation velocities $V_{2.2}$ of 100,150 and $200 \mathrm{~km} / \mathrm{s}$ at $r=2.2 r_{\mathrm{d}}$ have been used, respectively. See text for details.

as $3.2 r_{\mathrm{d}}$ in the case of the URC96. Throughout the following sections, the quantity $V_{\max }$ will refer to the usage of equation 5.5, whereas $V_{2.2}$ and $V_{\text {opt }}$ denote an input of the URC91 and URC96, respectively.

\subsubsection{The Modelling}

In the first step of the simulation, the twodimensional velocity field was generated on the basis of the respective intrinsic rotational law (either Eq. 5.5, 5.7 or 5.9), tilted and rotated according to the observed inclination and position angle of the respective galaxy. This was done by computing the line-of-sight rotation velocity $V_{\text {rot }}^{i}(r)$ at a projected position $(x, y)$ in the plane of the sky (with the kinematical center defined as $x=y=0$ ) via

$$
r^{2}=x_{t}^{2}+y_{t}^{2}
$$

with the transformation equations

$$
\begin{array}{r}
x_{t}=x \cos \delta+y \sin \delta, \\
y_{t}=(x \sin \delta-y \cos \delta) / \sin i .
\end{array}
$$

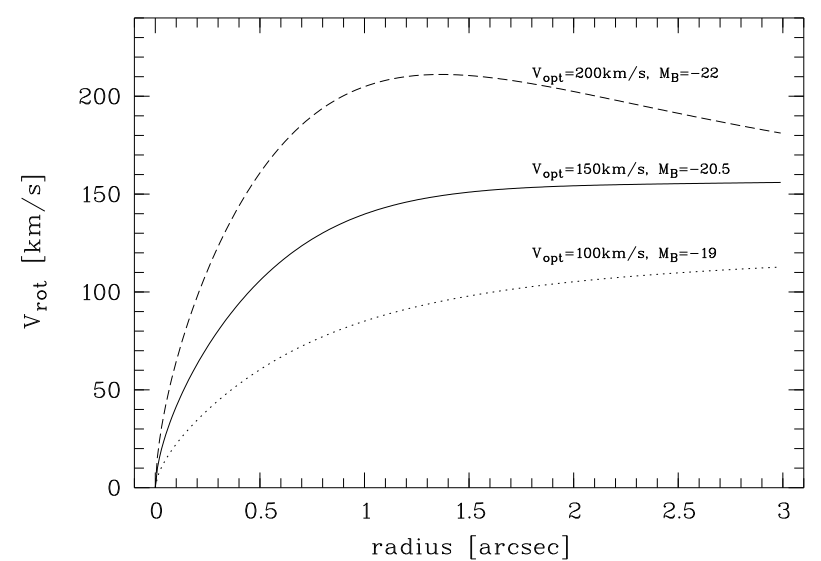

Figure 5.3: The Universal Rotation Curve (URC96) shape as introduced by Persic et al. (1996). A gas scale length of 0.6 arcsec has been assumed. The shape and, in particular, the velocity gradient in the outer regions, depend on the absolute magnitude. For clarity, different characteristic rotation velocities $V_{\text {opt }}$ of 100,150 and $200 \mathrm{~km} / \mathrm{s}$ at $r_{\mathrm{opt}}=3.2 r_{\mathrm{d}}$ have been used for the respective values of $M_{B}$.

By using the misalignment angle $\delta$ for the rotation of the simulated velocity field, the MOS slit position of the corresponding observed galaxy was parallel to the $X$ axis by definition.

This velocity field $V(x, y)$ was then weighted with the normalised surface brightness profile by calculating

$$
V_{\mathrm{w}}(x, y)=V(x, y) \times I_{\text {norm }}(x, y),
$$

where the profile $I_{\text {norm }}$ was normalized such that

$$
\int_{y 1}^{y 2} I_{\text {norm }}(x=\text { const }, y) d y=1, \quad \forall x .
$$

Here, $y 1$ and $y 2$ are the positions of the MOS slit edges along the spectral $(Y)$ axis, i.e. $y_{2}-y_{1}=1$ arcsec. The normalisation is mandatory to ensure that the integral of a constant velocity field would not be changed by the weighting.

To avoid the introduction of noise in the computation of Eq. 5.13, $I_{\text {norm }}$ was not derived from the observed but from the synthetic disk profile (chapter 4.3). The weighting took into account 
that, at a given position on the spatial $(X)$ axis in an observed 2-D spectrum, brighter regions on the spectral $(Y)$ axis contribute stronger to the velocity shift of an emission line.

Following this, the field was convolved according to the seeing during spectroscopy:

$$
V_{\mathrm{w}}(x, y) \stackrel{\mathrm{PSF}}{\longrightarrow} V_{\mathrm{wc}}(x, y)
$$

The Point Spread Function was assumed to be Gaussian with a FWHM determined from the mean DIMM values during the spectroscopy. These values had to be slightly increased depending on the redshifted wavelength of the emission line from which the observed RC was derived. The wavelength-dependent factor for this increase was determined by measuring the FWHM on sets of FDF images in $B, R$ and $I$, and comparing these values to the DIMM seeing values (which were automatically stored in the frame headers during the observations).

In the final computation step, a strip of one arcsecond width matching the position of the corresponding MOS slit was extracted from the velocity field $V_{\mathrm{wc}}(x, y)$ (see Fig. 5.4) and integrated perpendicular to the simulated slit, i.e., the projection of the velocity field strip onto the spatial axis was calculated:

$$
V_{\text {synth }}(r)=\int_{y 1}^{y 2} V_{\mathrm{wc}}(x, y) d y,
$$

where $y 1$ and $y 2$ again denote the slit egdes along the spectral axis and $r=x$ is the apparent galactocentric radius along the spatial axis. In all of the following, this position-velocity model $V_{\text {synth }}(r)$ will be referred to as the synthetic rotation curve, the term intrinsic rotation curve will denote the rotational law (e.g., Eq. 5.5) that is used as input for the modelling procedure. Note that the observed RC as described in Sect. 5.1 would directly reproduce the intrinsic RC only for a disk of $90^{\circ}$ inclination (perfectly edge-on) that is observed with an infinitely thin slit at infinitely large spatial and spectral resolution. In the simulation defined by Eqs. 5.10 to 5.16, the intrinsic quantity $V_{\max }$ (or, if the URC is

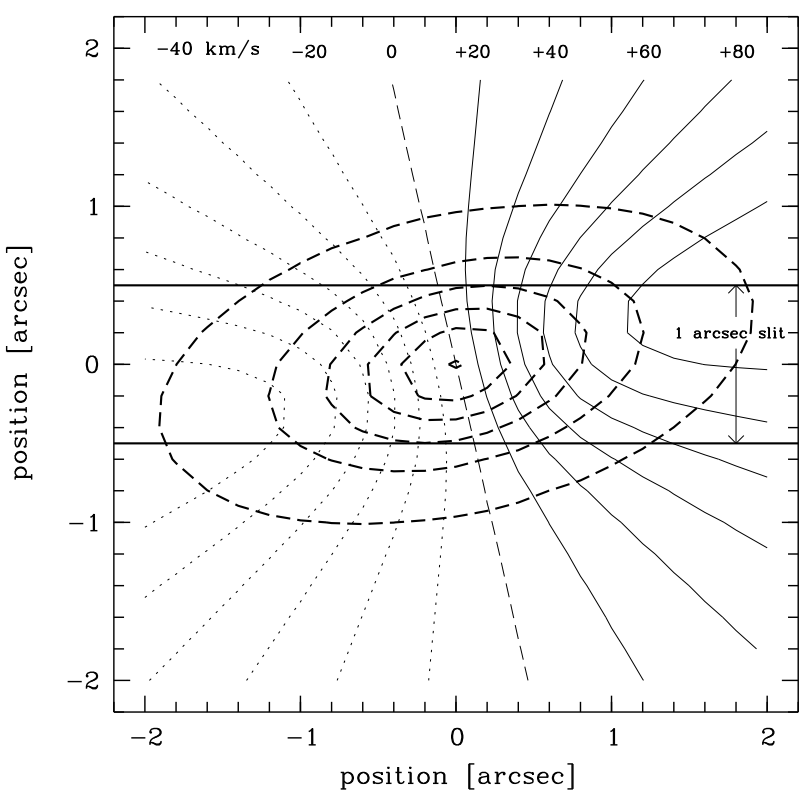

Figure 5.4: Simulated velocity field of an FDF spiral (catalogue ID \#4922) inclined by $i=64^{\circ}$ with an intrinsic rotation curve of $V_{\max }=156 \mathrm{~km} / \mathrm{s}$. Thin solid and dotted lines indicate iso-velocity zones ranging from $+120 \mathrm{~km} / \mathrm{s}$ to $-120 \mathrm{~km} / \mathrm{s}$ rotation in line-of-sight, i.e. comprising a factor $\sin i$. The thick horizontal solid lines denote the slit position during the MOS observation of the object. The thick dashed lines sketch the isophotes of the galaxy, with the outermost corresponding to $2 \sigma$ sky noise level in the $3000 \mathrm{~s}$ reference $I$-band image or $\mu_{I} \approx 25 \mathrm{mag} / \operatorname{arcsec}^{2}$. The misalignment between slit and apparent major axis is $\delta=+13^{\circ}$.

used as the intrinsic RC shape alternatively, the characteristic velocity $V_{2.2}$ or $V_{\text {opt }}$ ) is the only free parameter that tunes the reproduction of an observed RC by a synthetic curve. Only in the case of 3 FDF spirals, the gas scale length had to be kept as a second free parameter, since the values based on Eq. 5.6 were too large.

Each of the 113 observed RCs was visually inspected prior to the RC modelling. 7 RCs were not usable due to geometric distortions, of which 3 had too low an inclination (these originated from the 1999 pilot observation) and 4 had very large misalignment angles between $73^{\circ}$ and $80^{\circ}$. Two of the latter were initially selected as early- 
type galaxies, i.e., they were misclassified in a preliminary version of the photometric redshifts catalogue, the two others were secondary objects observed by chance. 15 curves featured large asymmetries or signatures of substantial kinematic disturbances. 7 spirals had a very low $\mathrm{S} / \mathrm{N}$ in the emission line(s), resulting in an apparent $\mathrm{RC}$ radius of less than one arcsecond or leading to an abortion of the fit. 3 "solid-body" rotators (cases of linearly increasing rotation velocity out to the last measured data point without any indication of a turnover) have not been used either. 4 Objects that did not show any rotation at all within the measurement errors were also excluded.

In total, $36 \mathrm{RCs}$ had to be rejected, while 77 spirals were appropriate for the $V_{\max }$ derivation (referred to as the TF sample). According to the visual inspection, the TF data set was subdivided into two categories of $\mathrm{RC}$ quality:

- A high quality sample of 36 RCs with a combination of very modest deviations from symmetry and relatively large spatial extent. In particular, these curves did not only show a "turnover" at large radii but clearly reached into a region of constant $V_{\text {rot }}^{\mathrm{o}}$ or only very small rotation velocity gradients. In effect, $V_{\max }$ could be measured robustly for these spirals and the TF analysis (next chapter) will be focussed on this subsample. 18 examples of high quality RCs are shown in Fig. 5.5.

- A low quality sample of 41 RCs which showed mild asymmetries or signatures of kinematic perturbations and had a relatively small spatial extent. Though the value of $V_{\max }$ could be derived for all these objects, the poor coverage of the "flat" RC region introduces very large relative errors for some of them (see next section). Furthermore, the maximum rotation velocity of a fraction of the low-mass spirals with low quality curves could be underestimated, which has an impact on the conclusions from the TF analysis, if these objects are included (see next chapter). 18 examples of low quality RCs are shown in Fig. 5.6.

Based on the RTF intrinsic RC shape, $V_{\max }$ was derived with two different methods:

1. by a visual comparison of synthetic and observed RC, and

2. via a $\chi^{2}$-fit of the synthetic to the observed $\mathrm{RC}$.

In the "by-eye" approach, it was aimed at best reproducing the observed RC by varying $V_{\max }$. If a perfect consistency at all radii could not be achieved, then only the outer $\mathrm{RC}$ regions were compared. Although a purely visual "fit" is not well-defined in a mathematical sense, it has the advantage that mild kinematic peculiarities do not affect the results. This topic will be further addressed and quantified below.

For the alternative approach, $\chi^{2}$ was defined as

$$
\chi^{2}=\sum_{r} \frac{\left[V_{\mathrm{rot}}^{\mathrm{o}}(r)-V_{\mathrm{synth}}(r)\right]^{2}}{\sigma_{v}(r)^{2}}
$$

and minimized in $V_{\max }$-space. Here, $\sigma_{v}(r)$ are the measured errors on $V_{\text {rot }}^{\mathrm{o}}(r)$ from the RC extraction.

The two methods are compared in Fig. 5.7. The sample of 77 spirals is subdivided with respect to the data quality: curves which clearly probe the region of constant rotation velocity at large radii are considered high quality $\left(N_{\text {obj }}=36\right)$, whereas RCs with smaller extent or slight asymmetries are included in the low quality subsample $\left(N_{\text {obj }}=41\right)$.

As can be deduced from Fig. 5.7, the by-eye "fits" and the $\chi^{2}$-fits are consistent within the errors for the majority of the objects. Nevertheless, a systematic trend towards lower values of $V_{\max , \chi^{2}}$ with respect to the visually derived values $V_{\text {max,vis }}$ is evident for slow rotators. An inspection of the observed RCs revealed that the discrepancies mainly arise in cases of asymmetric shapes. A further shortcoming of the $\chi^{2}$-fits is 

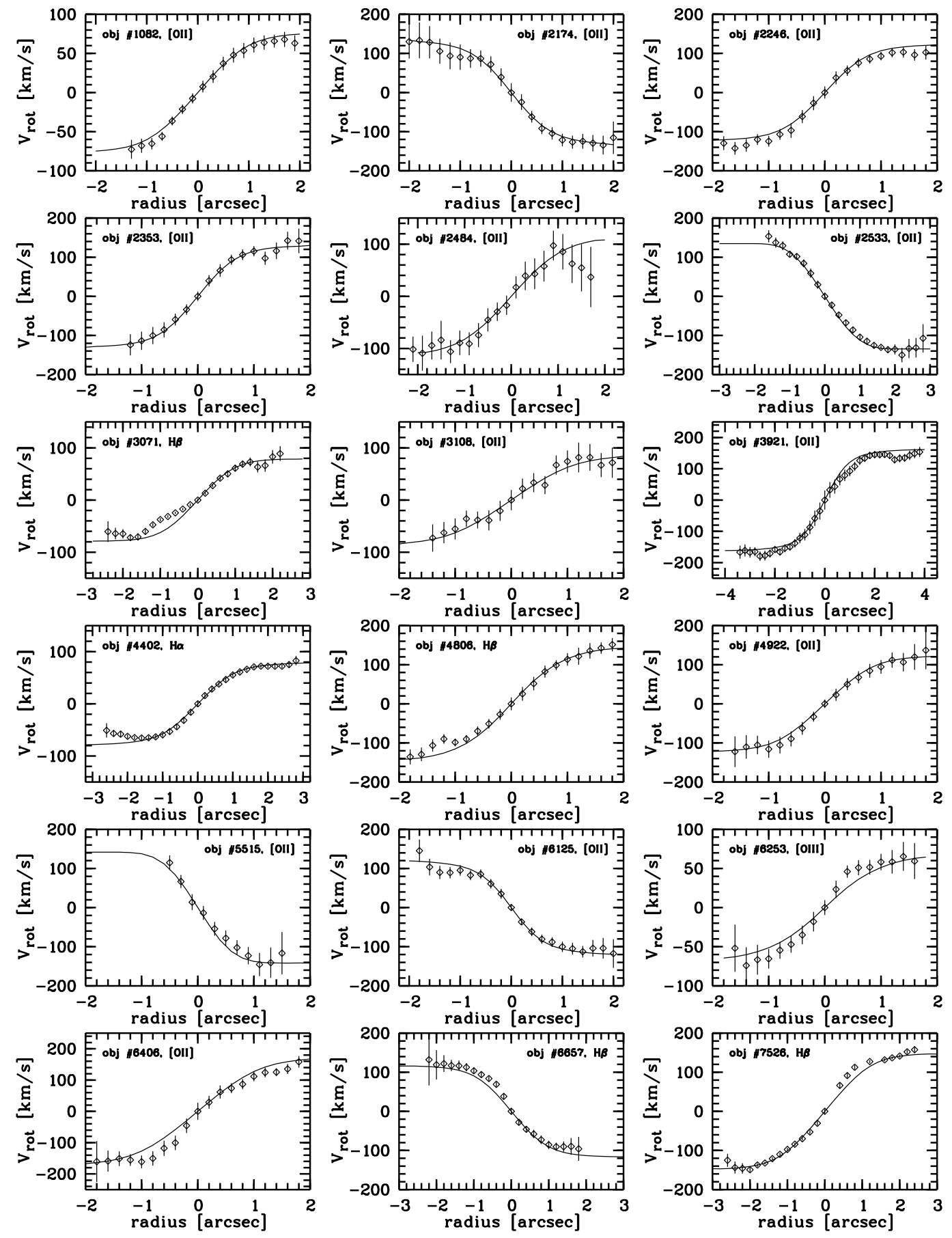

Figure 5.5: Examples of FDF spiral high quality rotation curves (RCs). Object number and fitted emission line are given for each spiral (either [O II] 3727, [O III] 5007, $\mathrm{H} \alpha$ or $\mathrm{H} \beta$ ). The solid lines denote the respective synthetic RCs which were created with an intrinsic "rise-turnover-flat" shape (Eq. 5.5). Note that the small spatial extent to the left hand side of the RC of galaxy \#5515 is due to contaminating light from a foreground elliptical. 

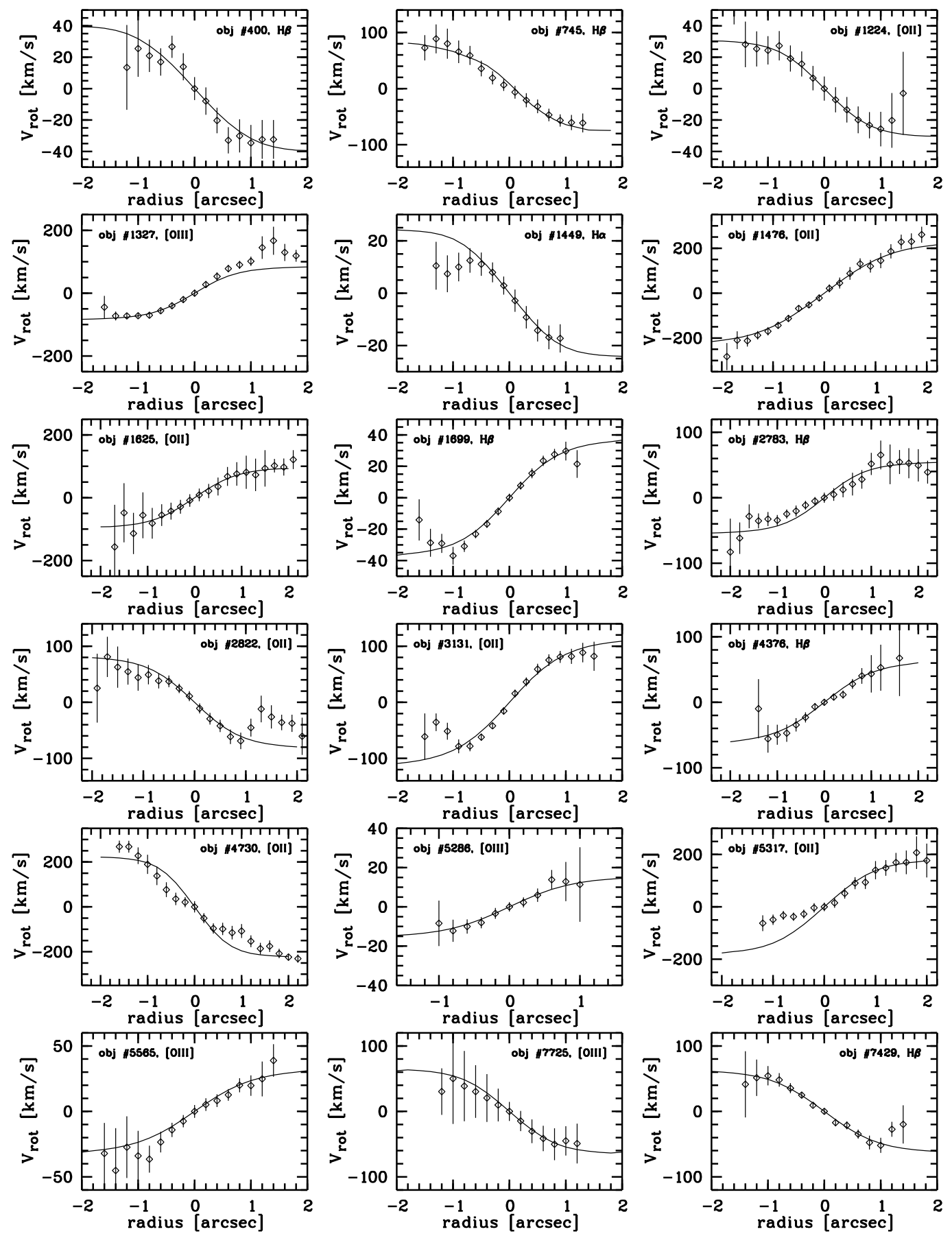

Figure 5.6: Examples of FDF spiral low quality rotation curves. Object number and fitted emission line are given for each spiral (either [O II] 3727, [O III] 5007, $\mathrm{H} \alpha$ or $\mathrm{H} \beta$ ). The solid lines denote the respective synthetic RCs which were created with an intrinsic "rise-turnover-flat" shape (Eq. 5.5). 


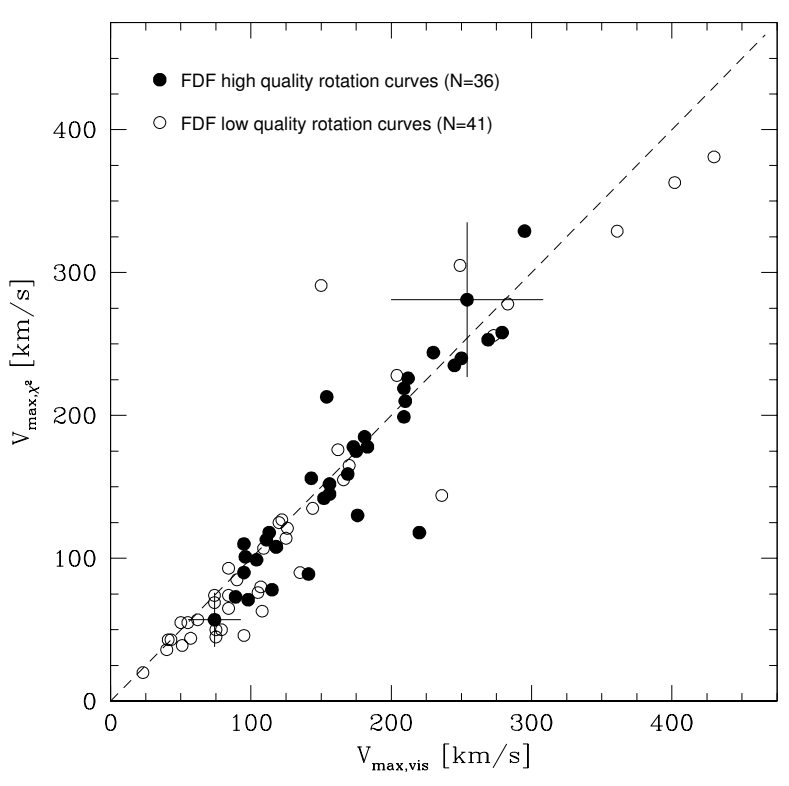

Figure 5.7: Comparison between the $V_{\max }$ derivation by visual alignment of observed and synthetic rotation curves ( $\mathrm{x}$ axis) and via $\chi^{2}$-fits (y axis). Typical error bars are shown for two objects.

that these are biased towards the inner parts of the curves by the higher $\mathrm{S} / \mathrm{N}$ of the emission lines at smaller radii. This is a disadvantage since the outer parts of an observed RC are the most robust source of $V_{\max }$ in the modelling procedure. Moreover, no correlation between the reduced $\chi^{2}$ values and the $\mathrm{RC}$ quality was found except for curves of almost perfect symmetry.

For these reasons, the visually derived values of $V_{\text {max }}$ will be the basis for the TF analysis. A further discussion of this topic is part of chapter 6.4.5.

\subsubsection{Error Budget}

The errors on $V_{\max }$ were computed according to $\sigma_{\mathrm{vmax}}^{2}=\sigma_{\chi}^{2}+V_{\max }^{2}(\tan i)^{-2} \sigma_{i}^{2}+V_{\max }^{2}(\tan \delta)^{2} \sigma_{\delta}^{2}$

Here, the first term on the right hand side is the error from the $\chi^{2}$-fits of the synthetic to the observed RCs (Eq. 5.17), covering the range $3 \mathrm{~km} / \mathrm{s} \leq \sigma_{\chi} \leq 59 \mathrm{~km} / \mathrm{s}$. The last two terms are the propagated errors of the respective uncertainties of the inclination and the misalignment angle. To derive these contributions from the errors $\sigma_{i}$ and $\sigma_{\delta}$, a simple geometric correlation between the observed and intrinsic rotation velocity was assumed,

$$
V_{\text {rot }}^{\mathrm{i}}=V_{\text {rot }}^{\mathrm{o}}(\sin i)^{-1}(\cos \delta)^{-1} .
$$

For the error propagation in $i$ follows

$$
\begin{array}{r}
\left(\sigma_{i} \frac{\partial}{\partial i} V_{\text {rot }}^{\mathrm{i}}\right)^{2}=\left(\sigma_{i} V_{\text {rot }}^{\mathrm{o}} \frac{\cos i}{\cos \delta \sin ^{2} i}\right)^{2} \\
=\left(\sigma_{i} V_{\text {rot }}^{\mathrm{i}} \frac{\cos i \cos \delta \sin i}{\cos \delta \sin ^{2} i}\right)^{2} \\
=\left(\sigma_{i} V_{\text {rot }}^{\mathrm{i}} \frac{\cos i}{\sin i}\right)^{2}=\left(V_{\text {rot }}^{\mathrm{i}} \tan ^{-1} i \sigma_{i}\right)^{2}
\end{array}
$$

and substitution of $V_{\text {rot }}^{\mathrm{i}}$ by $V_{\max }$ yields the second term on the right hand side of Eq. 5.18. The error propagation in $\delta$ similarly yields

$$
\begin{array}{r}
\left(\sigma_{\delta} \frac{\partial}{\partial \delta} V_{\text {rot }}^{\mathrm{i}}\right)^{2}=\left(\sigma_{\delta} V_{\text {rot }}^{\mathrm{o}} \frac{-\sin \delta}{\sin i \cos ^{2} \delta}\right)^{2} \\
=\left(\sigma_{\delta} V_{\text {rot }}^{\mathrm{i}} \frac{\sin \delta \cos \delta \sin i}{\sin i \cos ^{2} \delta}\right)^{2} \\
=\left(\sigma_{\delta} V_{\text {rot }}^{\mathrm{i}} \frac{\sin \delta}{\cos \delta}\right)^{2}=\left(V_{\text {rot }}^{\mathrm{i}} \tan \delta \sigma_{\delta}\right)^{2}
\end{array}
$$

and after substitution of $V_{\text {rot }}^{\mathrm{i}}$ by $V_{\max }$, the third term on the right hand side of Eq. 5.18 is gained. For the complete $\mathrm{TF}$ sample, the absolute and relative errors on $V_{\max }$ fall in the respective ranges $3 \mathrm{~km} / \mathrm{s} \leq \sigma_{\mathrm{vmax}} \leq 330 \mathrm{~km} / \mathrm{s}$ and $0.03 \leq \sigma_{\mathrm{vmax}} / V_{\max } \leq 0.91$, with a median $\left\langle\sigma_{\mathrm{vmax}} / V_{\max }\right\rangle=0.21$.

For the sub-sample of TF spirals with high quality RCs, the absolute and relative errors on $V_{\max }$ cover the respective ranges $3 \mathrm{~km} / \mathrm{s} \leq \sigma_{\operatorname{vmax}} \leq 135 \mathrm{~km} / \mathrm{s}$ and $0.03 \leq \sigma_{\mathrm{vmax}} / V_{\max } \leq 0.61$, with a median $\left\langle\sigma_{\mathrm{vmax}} / V_{\max }\right\rangle=0.19$.

A more detailed look into the $V_{\max }$ error budget is shown in Fig. 5.8. In plot a), the relative error $\sigma_{\mathrm{vmax}} / V_{\max }$ is displayed as a function of $V_{\max }$. For both classes of RC quality, the relative errors 

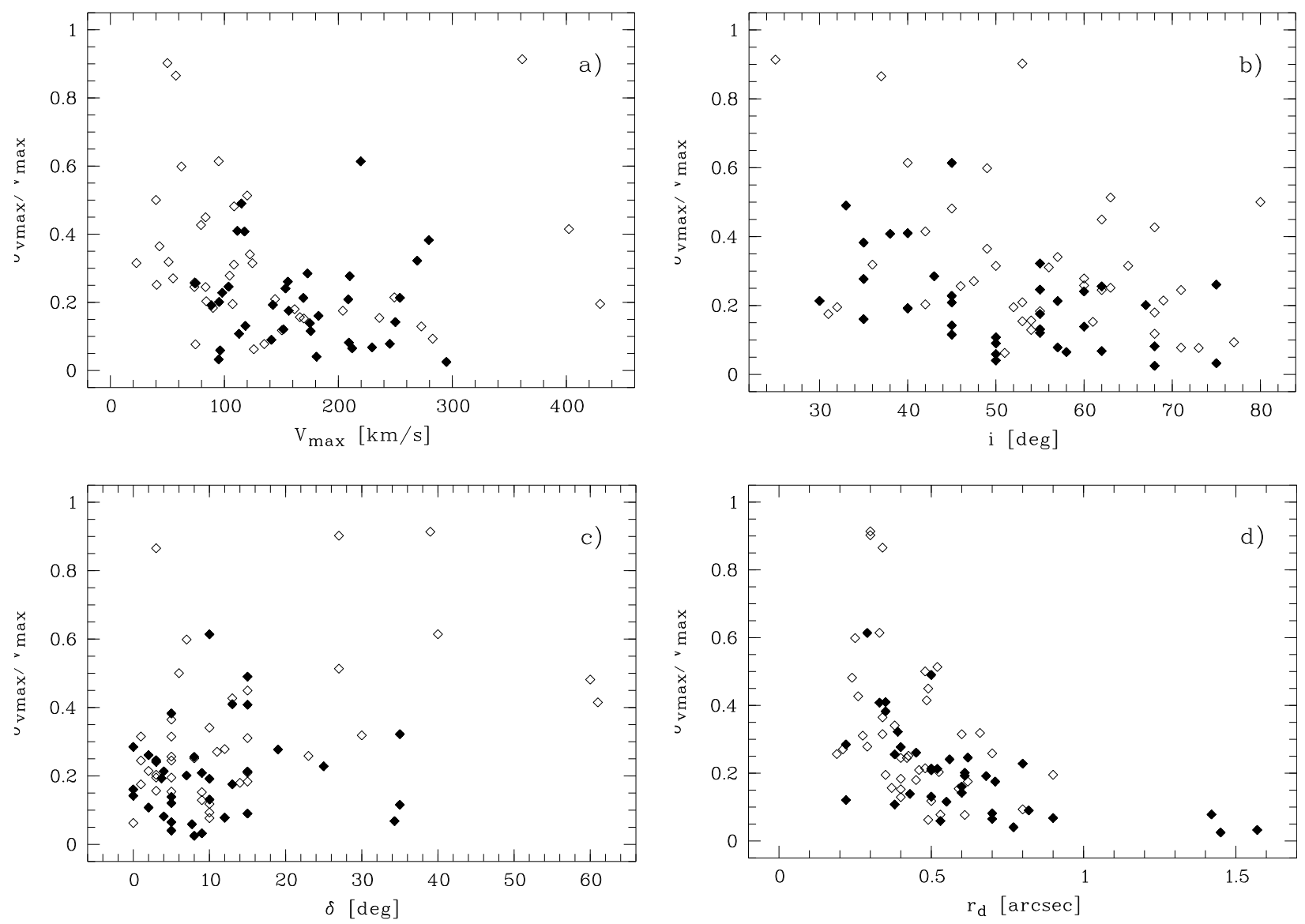

Figure 5.8: Relative errors on $V_{\max }$ plotted against various parameters. Filled symbols denote objects with high quality rotation curves and open symbols the low quality curves. Upper left figure: relative $V_{\max }$ error as a function of $V_{\max }$. Upper right figure: relative $V_{\max }$ error as a function of the inclination $i$. Lower left figure: relative $V_{\max }$ error as a function of the misalignment angle $\delta$. Lower right figure: relative $V_{\max }$ error as a function of the apparent disk scale length $r_{\mathrm{d}}$.

are larger in the slow rotator regime. Two effects probably act in combination here.

Firstly, an inspection of the RCs revealed that kinematic peculiarities are more frequent for slow rotators (low-mass spirals) of low data quality. This has an imprint on the error of the RC $\chi^{2}$-fits and thus on the total error $\sigma_{\text {vmax }}$.

A more subtle error contribution arises from the $\mathrm{RC}$ extraction. The errors of the row-by-row fits to an emission line basically depend on the $\mathrm{S} / \mathrm{N}$, not on the magnitude of the Doppler shifts due to rotation. Since the latter are on the average smaller for spirals in the low $-V_{\max }$ regime, the relative errors of the best-fitting central wavelengths become larger and so do $\sigma_{\chi}$ and $\sigma_{\text {vmax }}$. Fig. $5.8 \mathrm{~b}$ shows the dependence of the relative $V_{\max }$ error on the disk inclination. For decreasing values of $i$, the errors increase, because the best-fitting inclination values are less accurate for disks which are more face-on. On the other hand, the influence of the misalignment angle $\delta$ on the relative error $\sigma_{\mathrm{vmax}} / V_{\max }$ is relatively weak (Fig. $5.8 \mathrm{c}$ ).

The tight correlation between the relative error and the scale length $r_{\mathrm{d}}$ in Fig. $5.8 \mathrm{~d}$ may be a surprise at first sight because the scale length error propagation is not explicitly accounted for 
in $\sigma_{\text {vmax }}$. However, the sampling of the velocity field is lower for apparently small disks, hence the uncertainty of the $\mathrm{RC} \chi^{2}$-fit is larger.

\subsubsection{Comparison between Different Emission Lines}

For the majority of the FDF spirals, only one emission line per object could be used to extract an observed RC that was reliable for the $V_{\max }$ derivation procedure. Towards higher redshifts, this was due to the observed wavelength range which covered only the [O II] doublet, whereas $\mathrm{H} \beta$ or [O III] emission was redshifted out of the $\lambda$ window. Moreover, spectra of low $\mathrm{S} / \mathrm{N}$ mostly showed only one emission feature which was strong enough to extract an RC from it, even if multiple lines were detected.

In the case of 24 galaxies, roughly corresponding to $31 \%$ of the $\mathrm{TF}$ sample, more than one emission line was eligible for the derivation of $V_{\max }$. These galaxies are on the average brighter than the rest of the sample $\left(\langle R\rangle=20.81^{\mathrm{m}}\right.$ vs. $\left.\langle R\rangle=22.18^{m}\right)$, which partly can be attributed to the different redshift distributions $(\langle z\rangle=0.34$ vs. $\langle z\rangle=0.59)$. However, even if the sub-sample of spirals with only one usable emission line is restricted to a similar redshift range (using a constraint $z<0.44$ which yields $\langle z\rangle=0.34$ ), these galaxies have a median apparent brightness of $\langle R\rangle=21.98^{m}$. As expected, the 24 spirals with multiple usable emission lines represent objects with relatively high $\mathrm{S} / \mathrm{N}$ ratios.

For each TF object, the reference value of the maximum rotation velocity was based on the observed $\mathrm{RC}$ which had the highest quality in terms of radial extent, observed errors and symmetry. As a consistency check, it is worthwhile to compare $V_{\max }$ values based on different emission features.

RCs based on different emission lines are presented for 6 spirals in Fig. 5.9. The agreement between the individual curves ranges from fairly good to excellent. Straightforwardly, this is to be expected since all emission lines of a galaxy trace the same intrinsic velocity field. Therefore, the shape of the observed RCs should not depend on the used emission feature. Small deviations between the reference RC and the "secondary" curve may arise from different $\mathrm{S} / \mathrm{N}$ ratios of the respective emission lines. However, this effect could only induce discrepancies within the observed errors.

On the other hand, small-scale variations (e.g., due to density waves, see Sofue \& Rubin 2001) as well as mild perturbations of the velocity field due to a warp or a bar (ibid.) may cause deviating RCs if different emission features originate from different intrinsic positions. Since the MOS slit width corresponds to $\sim 5.7 \mathrm{kpc}$ at the mean redshift of the TF spirals, even clouds of emitting gas which are separated by several kpc (perpendicular to the slit direction) would not be kinematically resolved.

In terms of the observed errors, 21 out of the 24 curves from different lines were considered to be in agreement in a visual inspection. As an additional test, the $\chi^{2}$-based computation of $V_{\max }$ was alternatively applied to the secondary RCs. The results are shown in Fig. 5.10.

For 9 out of 24 spirals, the agreement between $V_{\max 1}$ (the reference value) and $V_{\max 2}$ (based on a secondary emission line) is better than $10 \%$. If the constraint is relaxed to $20 \%$ (which corresponds to the mean relative error of $V_{\max 1}$ ), then 15 out of 24 objects yield consistent results. It may therefore be concluded that RCs from different emission features agree within the errors for the majority of the galaxies. Hence it is probable that either i) different emission features probe similar regions of the intrinsic velocity field or ii) not only the observed velocity fields, which are smoothed by the PSF and the optical beam smearing, are rather symmetric, but also the intrinsic ones.

One additional statement can be made after this consistency check: The reference maximum rotation velocities $V_{\max 1}$ are on the average larger by $\sim 5 \%$ than the secondary values $V_{\max 2}$. Since 

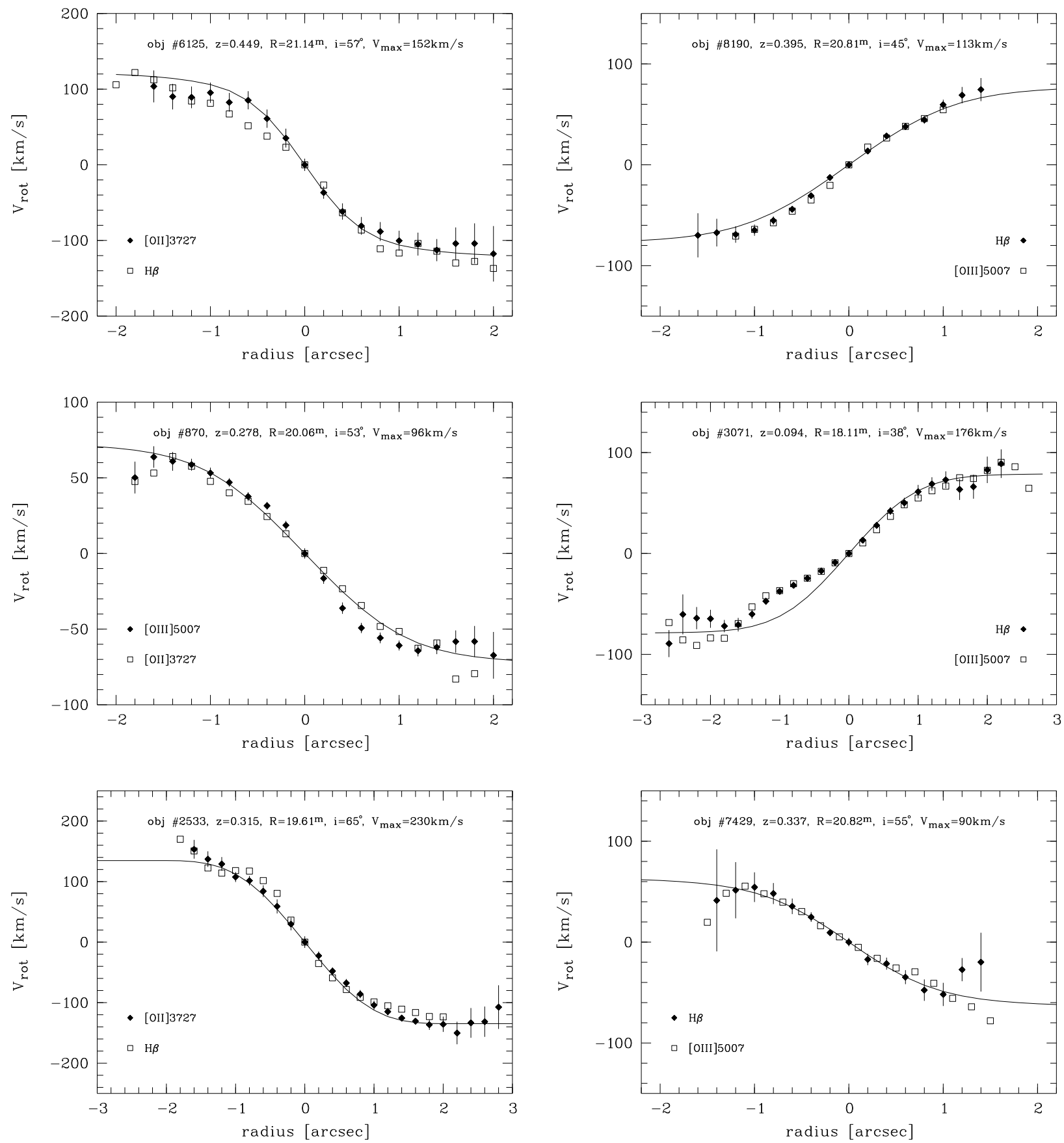

Figure 5.9: Comparison between observed rotation curves (RCs) derived from different emission lines. The RCs used as reference for the $V_{\max }$ derivation are denoted by solid symbols in all plots, the corresonding synthetic RCs are displayed as solid lines. The object ID, redshift, apparent $R$ magnitude, disk inclination, maximum rotation velocity and the used emission features are given for each spiral. For matters of clarity, errors on the measured rotation velocities are only shown for the reference curves. Secondary RCs extracted from other usable emission lines are shown as open symbols. Note that most of the secondary curves shown here have a radial extent similar to the reference RCs but feature larger errors (not displayed here). 


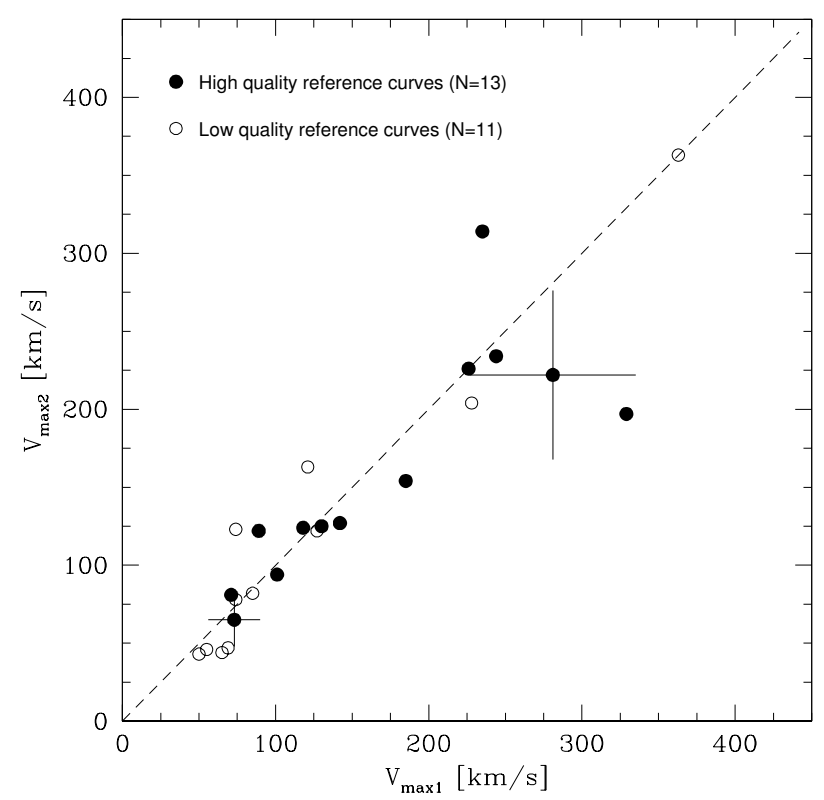

Figure 5.10: Comparison between maximum rotation velocities derived from different emission lines. The values used as reference (based on the line which yielded the rotation curve of the highest quality for a given spiral) are plotted along the x-axis, values derived from secondary emission features are given on the y-axis. Solid symbols denote reference rotation curves of high quality. Typical error bars are shown for two objects.

the corresponding RCs are of lower quality than the reference curves, this is evidence for slightly underestimated values yielded by the $\chi^{2}$-based computation of $V_{\max }$ in cases of low quality data.

\subsubsection{Comparison between Different Intrinsic RC Shapes}

Those previous studies which could rely on spatially resolved rotation curves mostly assumed an RTF shape for the intrinsic RC (e.g., Vogt et al. 1996, 1997, Barden et al. 2003). MilvangJensen et al. (2003) alternatively used the URC91 and found only marginal differences to the results yielded for an RTF shape, all within the RC analysis introduced by SP99. As stated above, the URC96 has not been used so far. Nevertheless, it is a more realistic parameteri-

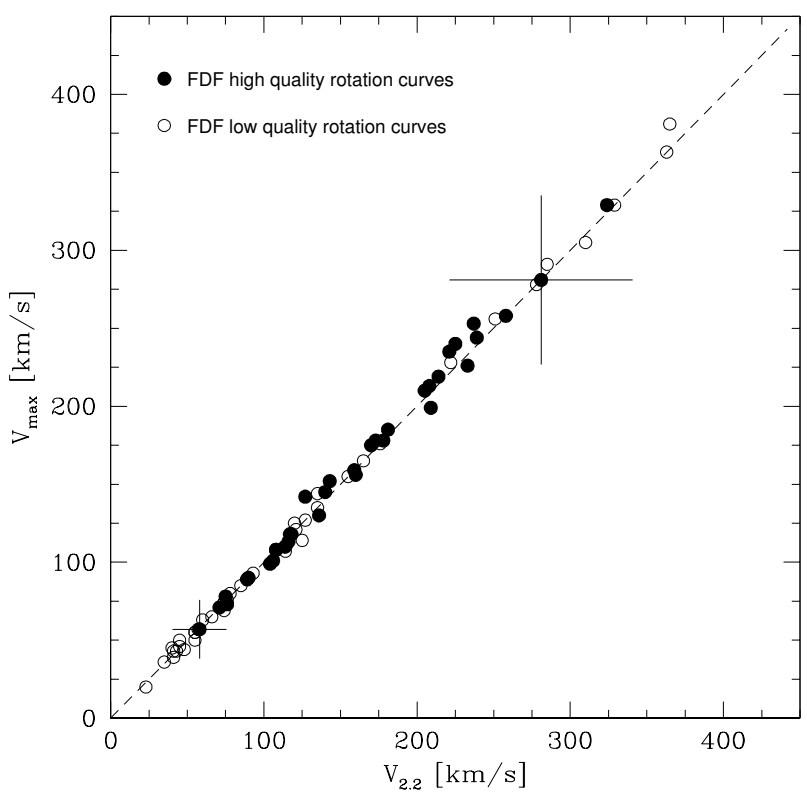

Figure 5.11: Comparison between rotation curve fitting with a simple "rise-turnover-flat" shape (yaxis) and the universal rotation curve shape as introduced by Persic \& Salucci (1991, x-axis). Solid symbols denote high quality curves which cover the region of constant rotation velocity at large radii. Typical error bars are shown for two objects.

sation than the URC91, in particular at small radii. The URC96 was derived on the basis of a local sample of $\sim 1100$ spirals. It will be checked now for the FDF sample whether the characteristic rotation velocity, which is given either as $V_{\max }, V_{2.2}$ or $V_{\text {opt }}$, is sensitve for to intrinsic $\mathrm{RC}$ shape. Since the velocity gradient at large radii is correlated to the absolute luminosity in the URC approach, whereas the RTF shape assumes a constant $V_{\text {rot }}$ for $r \gg r_{\mathrm{d}}$, differences should be likely.

In this test, the $\chi^{2}$-fits of the synthetic to the observed RCs have been performed for each of the three intrinsic shapes. Errors were computed with Eq. 5.18 separately for each method. In Fig. 5.11, the results from the RTF shape are compared to those of the URC91.

The values of $V_{\max }$ and $V_{2.2}$ are in agreement to within $5 \%$ for $79 \%$ of the FDF spirals and 


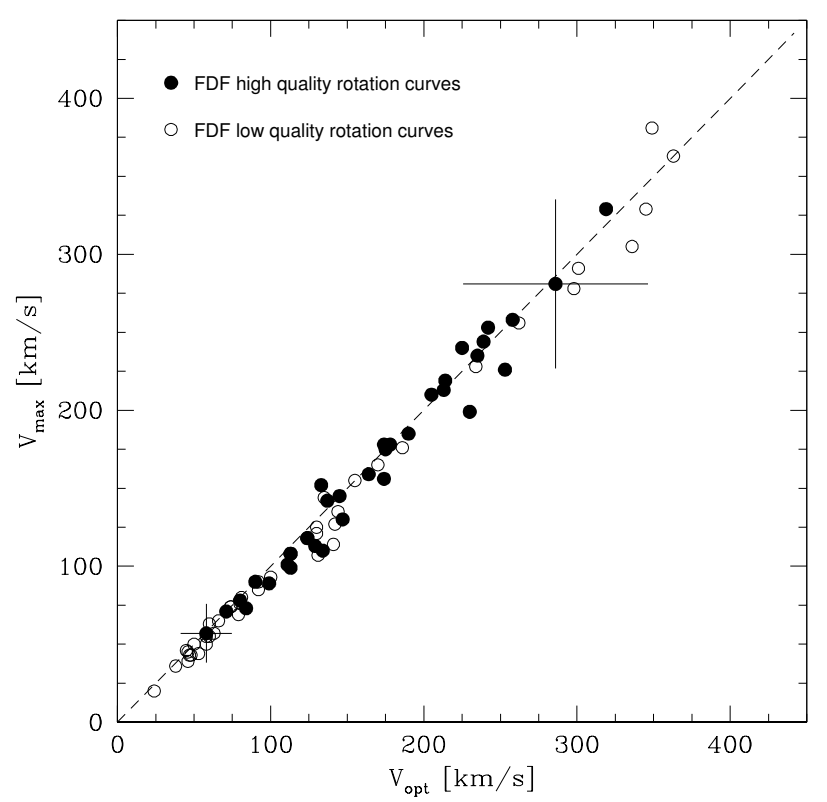

Figure 5.12: Comparison between rotation curve fitting with a simple "rise-turnover-flat" shape (y axis) and the universal rotation curve shape as introduced by Persic et al. (1996, x axis). High quality curves (solid symbols) cover the region of constant rotation velocity at large radii. Typical error bars are shown for two objects.

to within $10 \%$ for $95 \%$ of the galaxies, without a dependence on the velocity regime or the $\mathrm{RC}$ quality; the mean difference is $<1 \%$. This consistency is probably due to the small number of dwarfs or extremely luminous spirals in the TF sample; only one object has $M_{B}>-18$ and only three feature $M_{B}<-22$. Concerning the TF sample, the RTF and URC91 shapes can be considered equivalent, in compliance with the results of Milvang-Jensen et al. (2003), who also found marginal differences between the two.

A slightly larger spread in the deviations is seen between $V_{\max }$ and $V_{\text {opt }}$ (Fig. 5.12). Here, the fractional discrepancy has a scatter of 0.07 , while it was 0.04 for the comparison of $V_{\max }$ and $V_{2.2}$. The rotation velocities at $r_{\mathrm{opt}}=3.2 r_{\mathrm{d}}$ are on the mean larger by $4 \%$ than $V_{\max }$, with slightly increasing differences in the slow rotator regime. For the $10 \mathrm{TF}$ spirals with the smallest $V_{\max }$ val- ues $(<50 \mathrm{~km} / \mathrm{s})$, the median discrepancy is $10 \%$. This partly is an effect of the different characteristic radii in the two parameterisations of the universal rotation curve, which correspond to $2.2 r_{\mathrm{d}}$ for the URC91 and $3.2 r_{\mathrm{d}}$ for the URC96, respectively. Since the latter is larger than the former, the luminosity-dependent velocity gradient in the outer $\mathrm{RC}$ regions has a larger influence. Note that for a fraction of the FDF spirals, $V_{\text {opt }}$ is an extrapolation, since not all RCs extend out to $r=3.2 r_{\mathrm{d}}$.

However, even for the slowest rotators, the impact on the TF analysis is small: for an assumed TFR slope of -8 , even a difference in characteristic rotation velocity of $10 \%$ corresponds to an error in absolute magnitude of only $0.33^{m}$; this is within the $1 \sigma$ scatter of the local $B$-band relation (cf. Pierce \& Tully 1992). This topic will be further addressed in chapter 6.4.5.

\subsection{Distribution in $\mathrm{V}_{\max }$}

Prior to the TF analysis, it is important to check whether the maximum rotation velocities of the FDF spirals show a distribution different from that of local spirals. If indications of a kinematic evolution are found, than these would have to be taken into account in the attempts to quantify the evolution in luminosity with the TFR.

Two large local field spiral samples were used for this purpose. A study of 1097 spirals based on H I linewidth data from Haynes et al. (1999, Hay99 hereafter) and a data set of 2447 spirals with optical RCs by Mathewson \& Ford (1996, MF96 in the following).

The $V_{\text {max }}$-distribution of the complete FDF TF sample is compared to the local data sets in Fig. 5.13 a. To account for the differences in total numbers, the binning was set to $50 \mathrm{~km} / \mathrm{s}$ for the TF objects, $20 \mathrm{~km} / \mathrm{s}$ for Hay99 and $10 \mathrm{~km} / \mathrm{s}$ for MF96, respectively. Basically, the spread in $V_{\max }$ is similar between the three distributions. However, the $V_{\max }$-distributions of the large local samples are almost Gaussian, 

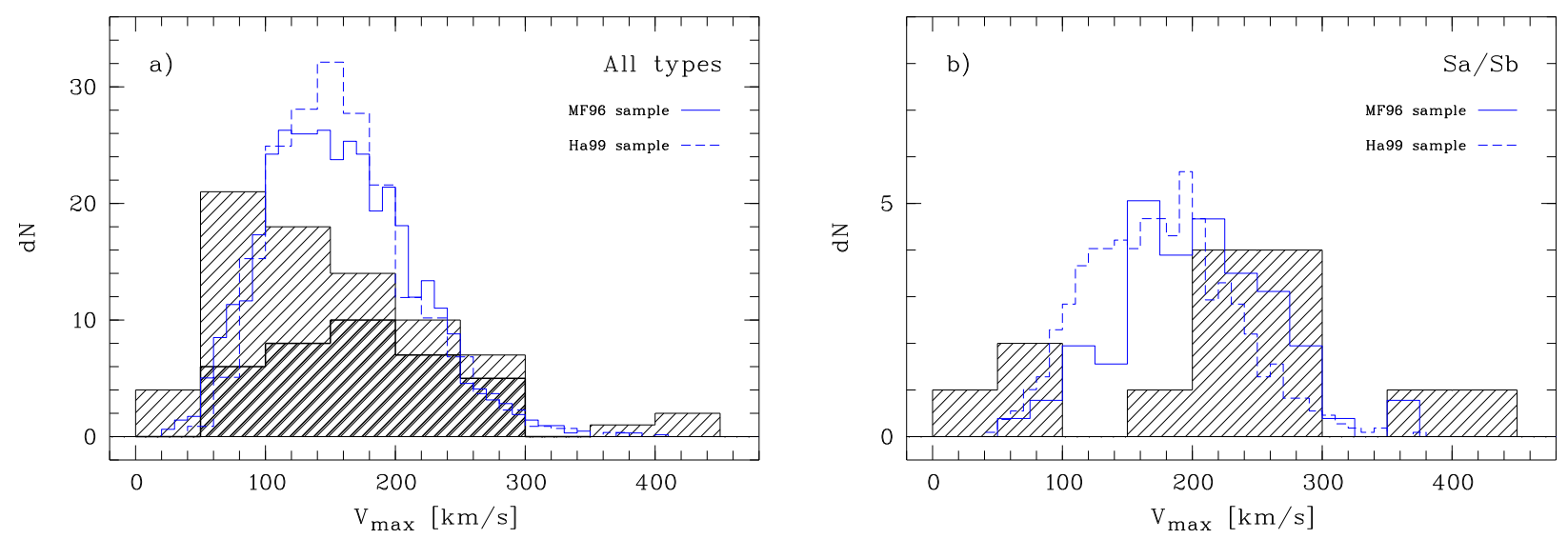

Figure 5.13: Distribution of $V_{\max }$ for the FDF spirals in comparison to two large local samples from Mathewson \& Ford (1996, MF96) and Haynes et al. (1999, Ha99). Left panel: Complete data sets comprising 77 (FDF TF sample, shaded histogram, bold histogram is rescricted to high quality rotation curves), 2447 (MF96) and 1097 (Ha99) spirals, respectively. The distributions of the high quality FDF sub-sample and the two local data sets are similar, see text for details. Right panel: Sub-samples of spirals earlier than $T \leq 3$, shaded histogram: FDF data (all qualities), this distribution obviously suffers from small number statistics $\left(N_{\mathrm{obj}}=14\right)$. Note that in all figures the binning and normalisation of the local samples differs from that of the FDF data to account for the different sample sizes.

while this is not the case for the FDF spirals - slow rotators seem to be slightly overrepresented here. In effect, the median in $V_{\max }$ is smaller for the latter: $\left\langle V_{\max }\right\rangle=125 \mathrm{~km} / \mathrm{s}$ $(\mathrm{FDF})$ vs. $\left\langle V_{\max }\right\rangle=152 \mathrm{~km} / \mathrm{s}$ (MF96) and $\left\langle V_{\max }\right\rangle=152 \mathrm{~km} / \mathrm{s}$ (Hay99). The high quality TF sample, on the other hand, does not show a larger fraction of slow rotators and the median of $163 \mathrm{~km} / \mathrm{s}$ differs by only $7 \%$ from the local samples.

A Kolmogorov-Smirnov test (e.g., Press et al. 1992) yields a maximum deviation between the cumulative distribution functions of $D=0.06$ (with a probability of only $P=0.04$ for a larger value) when Hay99 and MF96 are compared, confirming that these two samples are very similarly distributed. In a comparison between the local data sets and the FDF data, respective values of $D=0.26, P=0.01$ (MF96) and $D=0.21$, $P=0.07$ (Hay99) were found for the complete TF sample, indicating a fairly good agreement.

Figs. $5.13 \mathrm{~b}, 5.14 \mathrm{a}$ and $5.14 \mathrm{~b}$ show the distributions in $V_{\max }$ for the early-type, intermediate- type and late-type sub-samples. Here, criteria of $T \leq 3, T=5$ and $T \geq 8$ were used. Due to the small numbers for the FDF sub-sets, in particluar for the early- and late-types, a quantitative interpretation of these distributions in terms of a Kolmogorov-Smirnov test is not appropriate. The median values for the early types in the FDF, MF96 and Hay99 samples are 247, 204 and $174 \mathrm{~km} / \mathrm{s}$, respectively. At intermediate type (Sc), the agreement is much better, 145 (FDF), 134 (MF96) and $144 \mathrm{~km} / \mathrm{s}$ (Hay99) are the median values. For the sub-samples of very late types, these are $89 \mathrm{~km} / \mathrm{s}(\mathrm{FDF})$ and $85 \mathrm{~km} / \mathrm{s}$ (MF96), while the Hay99 sample only comprises two objects in this type range.

On the basis of the complete TF sample as well as the sub-samples for types later than Sc, one may thus conclude that the distant spiral data do not indicate an evolution of the rotation velocity in the flat regions of the RCs. Note that this would imply constant mean masses in the regime $0<z<1$ only if also the scale lengths were the same for distant and local spirals - 

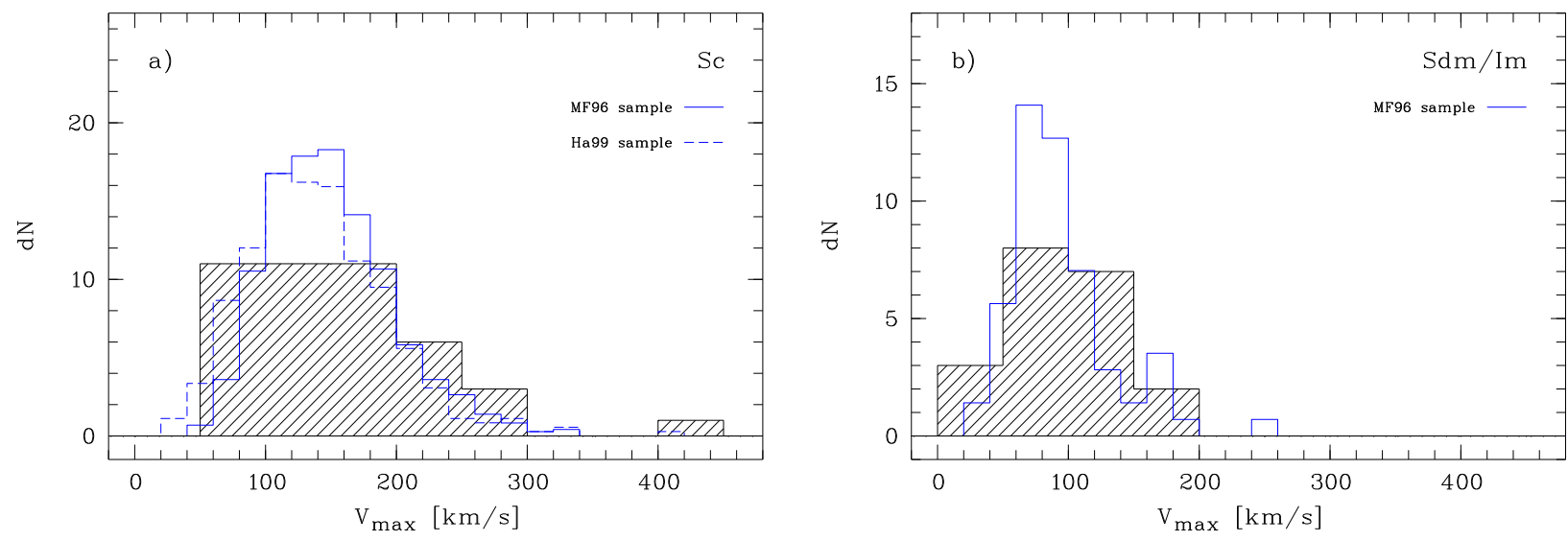

Figure 5.14: Distribution of $V_{\max }$ for the FDF spirals in comparison to two large local samples from Mathewson \& Ford (1996, MF96) and Haynes et al. (1999, Ha99). Left panel: Sub-samples of spirals with $T=5$, shaded histogram: FDF data (all qualities). Right panel: Sub-samples of spirals later than $T \geq 8$, shaded histogram: FDF data (all qualities). Only 2 objects from the Ha99 data fall into this type range and are not displayed. For intermediate and very late types, the distributions of the FDF and local samples are very similar, see text for details. Note that in all figures the binning and normalisation of the local samples differs from that of the FDF data to account for the different sample sizes.

this, however, is not observed, cf. chapters 1 and 6.3. Nevertheless, it is strengthened by the comparison given above that the FDF spirals make up a representative field sample and any deviation between local and distant TFR will have to be attributed to an evolution of the luminosity, not the kinematics.

Moreover, it has been revealed that the typedependence of $V_{\max }$, which is well known for spirals in the local universe (e.g., Rubin et al. 1985), also is valid at intermediate redshifts: earlier type spirals on the average are faster rotators than late-type spirals. As has been stated earlier, this observed correlation between spectrophotometric type and mass is not found in simulations within the CDM hierarchical model (e.g., Bell et al. 2003). To solve this problem, certain parameters of the inferred stellar population models were modified with, e.g., the colors of local spirals as a constraint. In other words, the simulations were calibrated with the presentday galaxy population (e.g., Boissier \& Prantzos 2001). Future theoretical studies will have to verify whether this strategy is also compatible with the almost unchanged $V_{\max }$-type correlation over the past 5 Gyrs (the look-back time corresponding to the mean redshift of the FDF TF spirals).

\subsection{Total Masses}

For a virialised system like the disk of a spiral galaxy, the rotation velocity is related to the mass. However, since the major contribution arises from the DM halo which introduces a linear increase of the mass with radius (see chapter 1.2.3), the estimation of the total mass is a non-trivial topic. An outer radius has to be defined that represents the boundary between a galaxy and its environment. Numerical simulations revealed that this so-called virial radius corresponds to a sphere of mean interior density $\bar{\varrho}=200 \varrho_{c}$, where $\varrho_{c}$ the critical density for closure of the universe (Cole \& Lacey 1996).

Van den Bosch (2003) constructed a sample of simulated galaxies and found that the mass within the virial radius, called the virial or total 


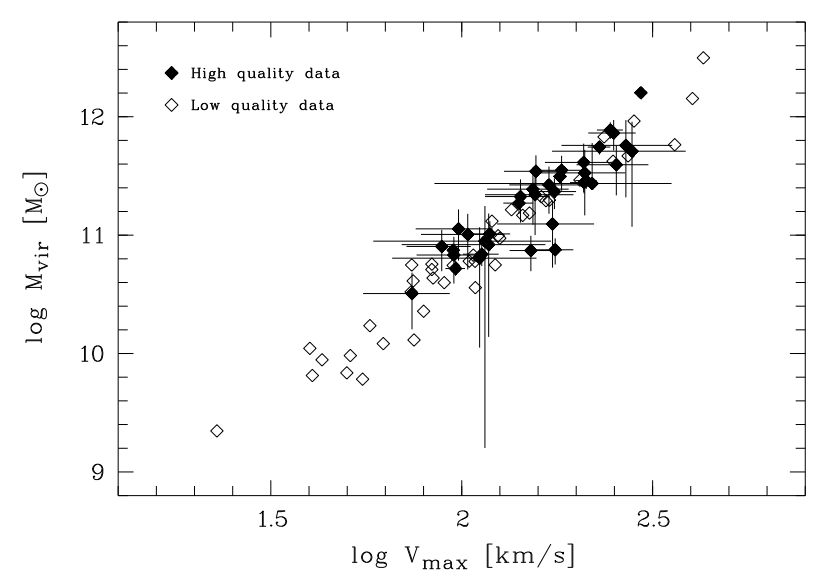

Figure 5.15: Virial masses of the FDF spirals as a function of the maximum rotation velocity. Filled symbols denote high quality rotations curves which reach well out into the region of constant rotation velocity. Errors are displayed only for these objects.

mass, can be well approximated via the equation

$$
M_{\mathrm{vir}}=2.54 \cdot 10^{10} M_{\odot}\left(\frac{r_{\mathrm{d}}}{\mathrm{kpc}}\right)\left(\frac{V_{\max }}{100 \mathrm{~km} / \mathrm{s}}\right)^{2} .
$$

The total masses of the FDF spirals were computed on the basis of the scale lengths from the surface brightness profile fits and the visually derived $V_{\max }$ values. The masses of the TF objects range from $2.2 \cdot 10^{9} M_{\odot}$ to $3.2 \cdot 10^{12} M_{\odot}$, with a median of $\left\langle M_{\text {vir }}\right\rangle=1.0 \cdot 10^{11} M_{\odot}$. Restricted to spirals with high quality rotation curves, the respective smallest and largest masses are $3.2 \cdot 10^{10} M_{\odot}$ and $1.6 \cdot 10^{12} M_{\odot}$, with a median of $\left\langle M_{\text {vir }}\right\rangle=2.3 \cdot 10^{11} M_{\odot}$. Errors on this mass estimations were computed via error propagation in $r_{\mathrm{d}}$ and $V_{\max }$ as

$$
\begin{aligned}
\sigma_{\text {Mvir }}^{2}= & 2.54^{2} \cdot 10^{20} M_{\odot}\left[\left(\frac{V_{\max }}{100 \mathrm{~km} / \mathrm{s}}\right)^{4} \sigma_{\mathrm{rd}}^{2}\right. \\
& \left.+4\left(\frac{r_{\mathrm{d}}}{\mathrm{kpc}}\right)^{2}\left(\frac{V_{\max }}{100 \mathrm{~km} / \mathrm{s}}\right)^{2} \sigma_{\mathrm{vmax}}^{2}\right]
\end{aligned}
$$

In Fig. 5.15, the virial masses are shown as a function of $V_{\max }$. The scatter due to the dependence on $r_{\mathrm{d}}$ is small, therefore it will be assumed in the following that a correlation between a

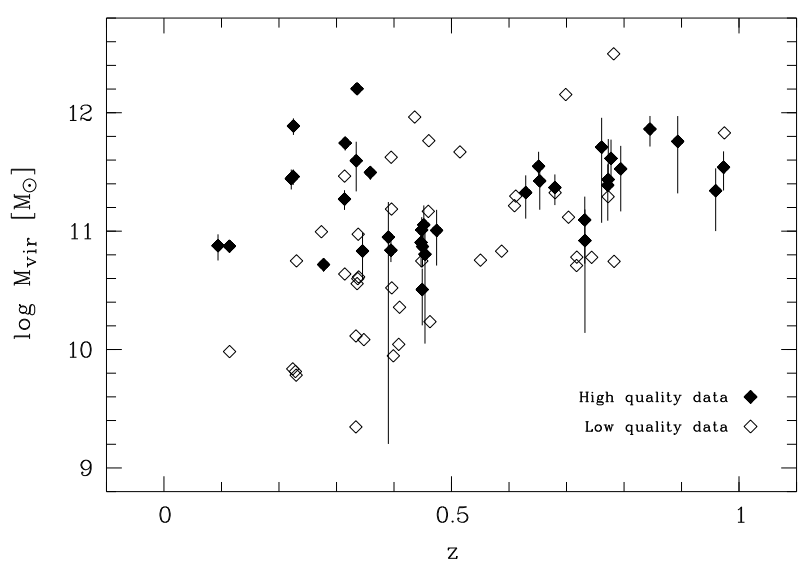

Figure 5.16: Virial masses of the FDF spirals as a function of redshift. The apparent increase of the masses with $z$ simply reflects the limiting magnitude $R \leq 23^{m}$ from the target pre-selection. Errors are displayed only for high quality data.

given parameter $f$ and $\log V_{\max }$ implies a correlation between $f$ and the total mass.

To illustrate the importance of the scaling relations for a distant spiral study, the virial masses are also shown as a function of redshift in Fig. 5.16. Apparently, the masses increase with increasing look-back time, in clear contrast to the predictions of the hierarchical merging scenario. This diagram, however, simply reflects the limiting magnitude of the sample. Since the targets for spectroscopy were pre-selected on apparent brightness $R \leq 23^{m}$, the corresponding lower luminosity limit increases with $z$ (see also chapter 4.7). More luminous spirals have, on the average, larger sizes, higher maximum rotation velocities and therefore higher masses.

To quantitatively test the predictions of the hierarchical scenario, it is mandatory to construct the scaling relations of the distant spirals. This will be done in the next chapter, which also comprises a broad discussion of the results of this thesis. 


\section{Chapter 6}

\section{Scaling Relations at Intermediate Redshift}

After the descriptions of the sample construction and the derivation of the spectrophotometric and kinematic data, the backbone of this thesis is going to be presented: the analysis of the distant scaling relations.

First of all, a sample of nearby spirals has to be chosen as a local reference (section 6.1). Then, the Tully-Fisher Relation (TFR) of the distant FDF spirals will be compared to that of spirals in the present-day universe (section 6.2). Similarly, the velocity-size relation can be used to test the evolution of the disk sizes (section 6.3). To rule out systematic errors in the interpretation of the results yielded by the distant scaling relations, a variety of tests were performed and will be described within section 6.4. These tests deal with the sample incompleteness, the color-residual relation, environmental effects, star formation rates, intrinsic $\mathrm{RC}$ shapes, different methods of intrinsic absorption corrections and the groundbased imaging. In section 6.5 , it will be shown that the TFR derived with the FDF spirals offers a simple explanation for the discrepancies between different previous observational studies of distant spirals. A brief comparison to the predictions of stellar population models is then outlined in section 6.6. The results will be interpreted in the framework of the hierarchical merging scenario in the last section. For a data table on the results presented in this chapter, see appendix C.
Some aspects of the analysis in this chapter have been presented briefly in Böhm, Ziegler \& Fricke (2001), Ziegler et al. (2002), Böhm et al. (2003a, 2003b).

\subsection{The Local Reference}

To be able to derive the spectrophotometric and/or kinematic evolution of spirals at intermediate redshifts, it is crucial to carefully choose a data set of spirals at low $z$ that can be used for reference. Consistency between the distant and local data sets in terms of the intrinsic absorption correction is one of the key issues, see further below. As stated in chapter 1, a large number of local samples have been constructed during the last decade, using kinematic data based on radio observations or optical spectra.

In this thesis, the term "local" refers to redshifts below $z \leq 0.05$ corresponding to systematic velocities $V_{\text {sys }} \leq 15000 \mathrm{~km} / \mathrm{s}$. None of the TFR studies which focussed on nearby spirals comprised objects at higher redshifts. Since a redshift $z=0.05$ corresponds to a look-back time of only $\sim 0.7$ Gyrs, or $\sim 5 \%$ of the age of the universe, galaxies within this limit can be considered to represent the same cosmic epoch, i.e., the present-day universe.

Most of the previous studies on the distant TFR (e.g., Milvang-Jensen et al. 2003, Vogt et al. 1996, 1997, Vogt 2000) used the sample from 
Pierce \& Tully (1992, PT92 hereafter) as a local reference. To achieve a direct comparability with the results of these authors, the PT92 data will be used as a comparison sample for the major parts of the following analysis. This sample consists of $\sim 50$ spirals at redshifts $z<0.005$ with $V_{\max }$ measurements from $\mathrm{H}$ I linewidth data. For 15 of the galaxies, distance estimates were computed, e.g., using Cepheids. The $B$-band TFR of the PT92 sample, corrected for intrinsic absorption following Tully \& Fouqué (1985, TF85 hereafter), is

$$
M_{B}=-7.48 \log V_{\max }-3.10 .
$$

As described in chapter 4.6.5, the TF85 approach yields an inclination-dependent rest-frame $B$ absorption $A_{B}^{i}$ which can be computed in two different conventions, either with zero faceon $\left(i=0^{\circ}\right)$ extinction or a face-on value of $A_{B}^{0}=0.27^{m}$. Since the latter accounts for a non-negligible dust disk thickness and is thus more realistic, it has been adopted in this thesis. However, PT92 used $A_{B}^{0}=0^{m}$, and their zero point of $b=-3.10$ therefore has to be modified to achieve consistency with the FDF data:

$$
b_{n}=b-A_{B}^{0}=-3.10-0.27=-3.37 .
$$

Furthermore, the original PT92 relation was affected by a slight calibration error (Kannappan 2003, priv. com.). In a later study (Tully \& Pierce 2000, TP00 hereafter), 5 out of the 15 galaxies with distance estimates from the initial data set have been re-calibrated. To derive the amount of the calibration error, the publicly available electronic tables of the distance estimators from PT92 and the TP00 objects have been combined. The absolute magnitudes of the five calibrators were compared via

$$
\Delta M_{B}^{\mathrm{cal}}=M_{B}^{\mathrm{pt} 92}+A_{B}^{i}-\left(M_{B}^{\mathrm{tp} 00}+A_{B}^{i, W}\right) .
$$

Here, the different approaches for the intrinsic absorption correction have been taken into account: $A_{B}^{i}$ gives the extinction following TF85,
$A_{B}^{i, W}$ refers to the convention by Tully et al. (1998, see chapter 4.6.5 and section 6.4.6 further below) which has been used by TP00.

On the mean, the re-computed calibrators from TP00 were brighter by $\left\langle\Delta M_{B}^{\text {cal }}\right\rangle=-0.15^{m}$. This offset also has to be added to the PT92 zero point:

$$
b_{n 2}=b_{n}+\left\langle\Delta M_{B}^{\mathrm{cal}}\right\rangle=-3.37-0.15=-3.52 .
$$

Hence, the local PT92 $B$-band TFR becomes

$$
M_{B}=-7.48 \log V_{\max }-3.52,
$$

with an observed scatter of $\sigma_{B}=0.41^{m}$.

Unfortunately, the complete PT92 sample is not electronically available. In particular, the disk sizes have not been published by the authors. For an analysis of the distant velocity-size relation, the sample from Haynes et al. (1999, Hay99 hereafter) was selected as a second local reference.

This sample includes 1196 spirals in total at a median redshift $\langle z\rangle=0.015,70 \%$ of which are type Sc, similar to the FDF TF sample which has an Sc fraction of $56 \%$. $V_{\max }$ values were derived from radio observations and classified by the authors according to the H I profile quality. In the following, only the 1097 objects with good quality radio data will be considered.

The photometric data on the Hay99 objects only comprised $I$-band values of the apparent magnitudes, galactic extinctions, disk sizes etc. To perform a consistency check between the TFRs of the Hay99 and PT92 samples in the $B$-band, the apparent magnitudes $m_{I}$ were transformed to absolute magnitudes $M_{B}$ with the equation

$$
M_{B}=m_{I}-A_{I}^{g}+(B-I)_{\mathrm{fg} 94}-A_{B}^{i}-D M(z) .
$$

Here, $A_{I}^{g}$ is the Galactic absorption as given by Hay99, $(B-I)_{\mathrm{fg} 94}$ is the type-dependent color index $B-I$ as given by Frei \& Gunn (1994), $A_{B}^{i}$ is the correction for intrinsic $B$-band absorption following TF85 and $D M(z)$ is the distance 


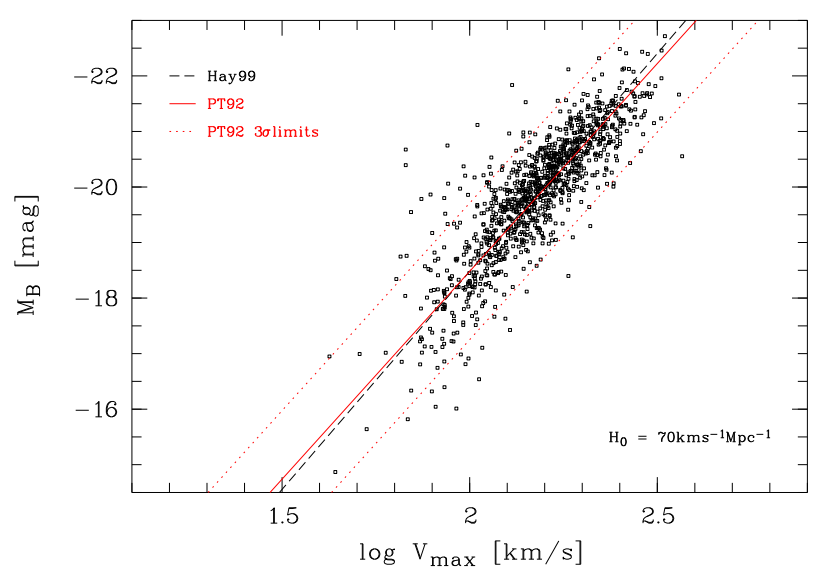

Figure 6.1: Consistency check between the two local data sets from Haynes et al. (1999, Hay99) and Pierce \& Tully (1992, PT92). The individual objects of the Hay99 sample are denoted by the small squares, the solid and dashed lines indicate the respective TFRs. For the PT92 sample, the $3 \sigma$ limits are additionally shown. The two relations are in good agreement.

modulus for a redshift $z$. The latter was calculated from the systematic velocities with respect to the Cosmic Microwave Background as given by Hay99, assuming a Hubble constant of $H_{0}=70 \mathrm{~km} \mathrm{~s}^{-1} \mathrm{Mpc}^{-1}$.

A bisector fit (a combination of two least-square fits with the dependent and independent variables interchanged) to the Hay99 sample yields

$$
M_{B}=-7.85 \log V_{\max }-2.78 .
$$

Fig. 6.1 illustrates the comparison to the TFR by PT92. The two relations are perfectly consistent in the regime $V_{\max } \approx 100 \mathrm{~km} / \mathrm{s}$ and show only a small offset of $\Delta M_{B} \approx 0.18^{m}$ at $V_{\max }=300 \mathrm{~km} / \mathrm{s}$.

This very good agreement, in particular within the $V_{\max }$ range covered by the high quality FDF TF sample (between $74 \mathrm{~km} / \mathrm{s}$ and $295 \mathrm{~km} / \mathrm{s}$ ) is a strong argument for the reliability of using the PT92 sample for the TF analysis and the Hay99 sample for the study of the velocity-size relation.

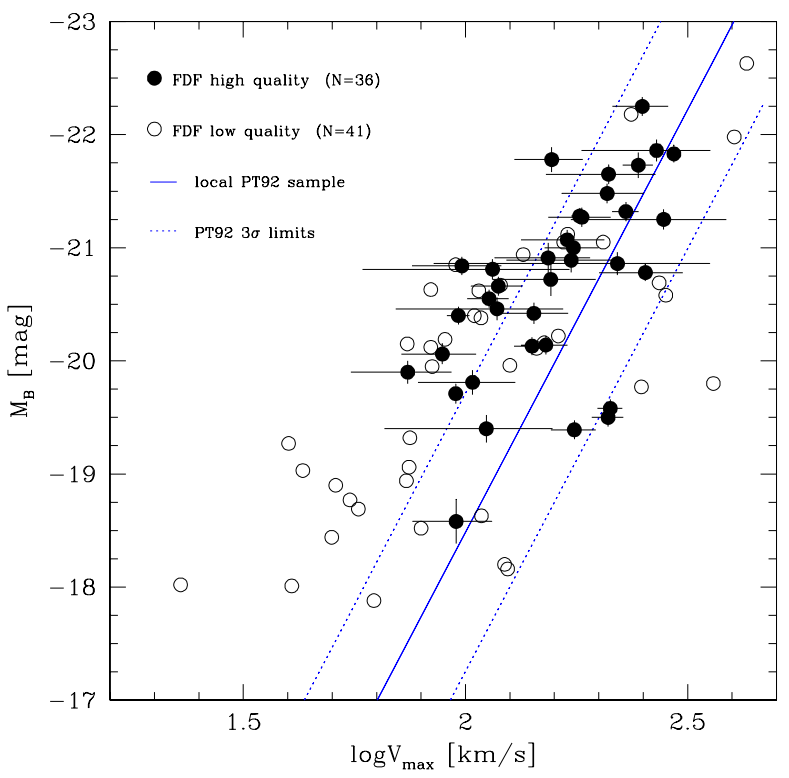

Figure 6.2: FORS Deep Field (FDF) sample of spirals in the range $0.1 \leq z \leq 1.0$ in comparison to the local TFR by Pierce \& Tully (1992, PT92). The different symbols denote the rotation curve quality of the distant FDF spirals: high quality curves (solid circles) extend well out to the region of constant rotation velocity at large radii, open symbols represent data of lower quality.

\subsection{The Distant Tully-Fisher Relation}

Finally, all the preparing steps have been taken to enter the TF analysis. In Fig. 6.2, the rotation velocity-luminosity diagram of the FDF sample is shown, along with the local TFR from PT92. As in the last chapter, the FDF sample is sub-divided according to the rotation curve (RC) quality. In the case of the high quality data, the curves have a sufficient spatial extent to probe the region of constant rotation velocity at large radii, thereby yielding robust values of $V_{\max }$. For the low quality data, on the other hand, one cannot rule out the possibility that $V_{\max }$ is underestimated for a fraction of the objects. In effect, offsets from the local TFR towards higher luminosities could be mistakenly 
derived. This issue will be addressed in more detail further below.

To rest the analysis on the most robust basis, only the high quality data will be used in most of the following. However, when certain sub-samples have to be considered (like, e.g., spiral sub-types), these will be drawn from the complete data set to achieve better statistics. In the parameter space of the TF diagram, the high quality objects cover the ranges $-18.6 \geq M_{B} \geq-22.2$ in absolute magnitude and $74 \mathrm{~km} / \mathrm{s} \leq \log V_{\max } \leq 295 \mathrm{~km} / \mathrm{s}$ in maximum rotation velocity.

For a given FDF object with corresponding values for $V_{\max }$ and $M_{B}$, the offsets from the local TFR can be calculated following

$$
\Delta M_{B}=7.48 \log V_{\max }+3.52+M_{B} .
$$

As previously mentioned, distant spirals are likely to incorporate stellar populations which are on the average younger than those of spirals in the local universe. In effect, the luminosities of distant spirals should be higher for a given mass (or a given $V_{\max }$ ). A test of this simple hypothesis is visualised in Fig. 6.3, where the TF offsets as defined in Eq. 6.8 are shown as a function of redshift.

One striking feature of the TF offsets is their large scatter $\sigma_{\Delta M}=1.32^{m}$. When only the high quality objects are considered, the value $\sigma_{\Delta M}=0.97^{m}$ is still more than a factor of two larger than is observed locally; PT92 found $\sigma_{B}=0.41^{m}$ for their sample. It also is substantially larger than is expected from the errors on the maximum rotation velocity: under the assumption of the PT92 TFR slope, $a=-7.48$, these errors $\sigma_{\text {vmax }}$ would correspond to a propagated error of $\sigma_{\Delta M}=0.63^{m}$ in the TF offsets, this is a factor of $\sim 1.5$ smaller than the observed value.

Two effects probably act in combination here. Firstly, the intrinsic scatter of the distant TFR could be increased with respect to the local one. In other words, the large value of $\sigma_{\Delta M}$ may

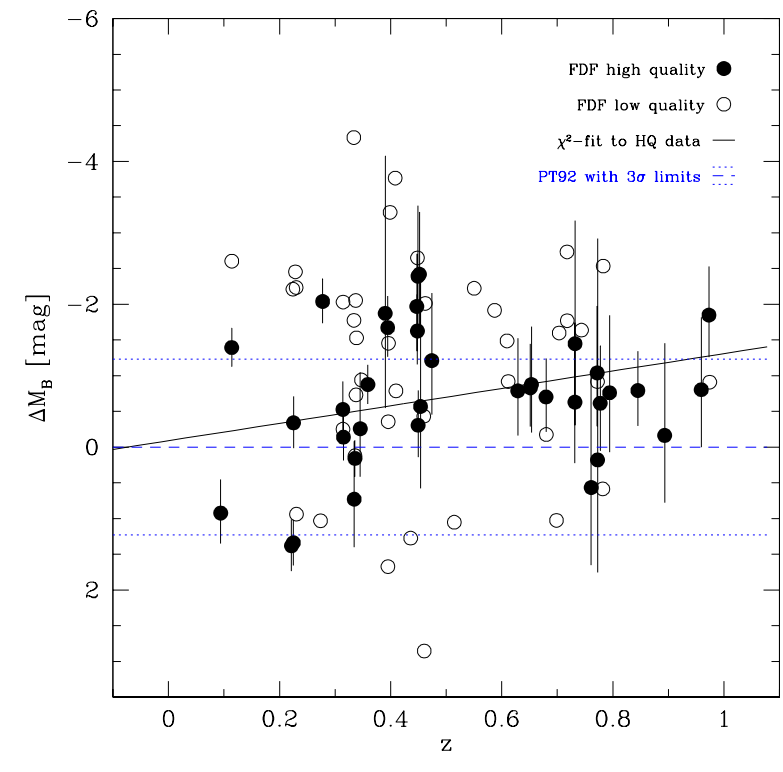

Figure 6.3: Offsets of the FORS Deep Field sample from the local TFR by Pierce \& Tully (1992, PT92) as a function of redshift. Filled symbols denote high quality rotation curves which extend well out to the region of constant rotation velocity at large radii. The dashed line indicates a zero offset, the dotted lines correspond to the $3 \sigma$ limits of the local TFR.

not be due to the spatial and geometric limitations to the accuracy of the $V_{\max }$ measurements, which have been quantitatively considered as described in chapter 5.2.3, but an evolution in the TF parameter space. E.g., the star formation efficiencies of the FDF galaxies may cover a broader range than those of their local counterparts. This would correspond to an increased spread of their mass-to-light ratios and, in turn, a larger scatter of the distant TFR.

Prior to a discussion of the second, more fundamental effect, it shall be tested whether the TF offsets show a correlation with the look-back time $\left(t_{0}-t_{1}\right)$. In the regime $z \leq 1$, this time can be approximated by $z \approx t_{0}-t_{1}$ (e.g., Carroll \& Press 1992). A linear $\chi^{2}$-fit to the high quality data yields

$$
\Delta M_{B}=-(1.22 \pm 0.56) \cdot z-(0.09 \pm 0.24) .(6.9)
$$


For this fit, the error of the offsets was computed as

$$
\begin{aligned}
\sigma_{\Delta \mathrm{TF}}^{2} & =\sigma_{M \mathrm{fdf}}^{2}+7.48^{2} \sigma_{\operatorname{logvfdf}}^{2} \\
& +\sigma_{M_{\mathrm{pt}}}^{2}+7.48^{2} \sigma_{\operatorname{logvpt}}^{2}
\end{aligned}
$$

Here, the first and third terms are the errors in absolute $B$ magnitude for the FDF and PT92 data, while the second and fourth terms are the propagated errors from the uncertainties in $\log V_{\max }$ for the FDF spirals and the PT92 sample, respectively. Since individual errors were not available for the PT92 data, mean errors of $\sigma_{\mathrm{mb}}=0.04^{m}$ on the photometry, of $\sigma_{\mathrm{dm}}=0.15^{m}$ on the distance modulus and of $\sigma_{\text {logvpt }}=0.021$ were assumed, as given by the authors. The resulting error in $M_{B}$ is

$$
\sigma_{M_{\mathrm{pt}}}^{2}=\sigma_{\mathrm{mb}}^{2}+\sigma_{\mathrm{dm}}^{2}
$$

As can be deduced from Eq. 6.9, an increasing brightening with rising look-back time is observed, as expected under the assumption of decreasing mean ages of the stellar populations. Due to the large scatter, however, the significance is only $\sim 2 \sigma$. Within the errors, this result is in agreement with the finding of MilvangJensen (2003), who derived a single-parameter fit

$$
\Delta M_{B}=-(0.9 \pm 0.3) \cdot z
$$

for $\Lambda=0, q_{0}=0.05$ and $H_{0}=75 \mathrm{~km} \mathrm{~s}^{-1} \mathrm{Mpc}^{-1}$. Note that this correlation would be slightly steeper in the cosmology adopted in this thesis, cf. chapter 4.6.3.

Besides the dependence of $\Delta M_{B}$ on redshift, the comparison of our sample with PT92 in Fig. 6.2 also implies a correlation between the $\mathrm{TF}$ offsets and the maximum rotation velocities. Even when only the high quality RCs which probe the "flat" region at large radii are considered, a number of distant spirals in the regime $V_{\max } \approx 100 \mathrm{~km} / \mathrm{s}$ are overluminous with $>3 \sigma$ confidence, given the observed scatter of $0.41 \mathrm{mag}$ for the local sample. This may indicate a slope change in the TFR between the local

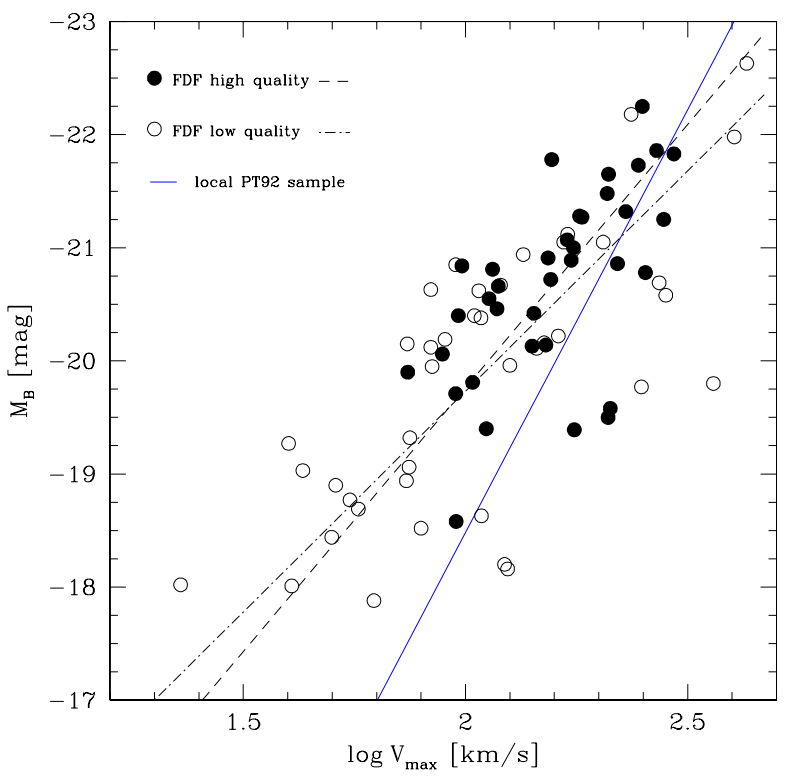

Figure 6.4: Separate bisector fits to the FDF high quality data (dashed line) and low quality data (dotdashed line), respectively, compared to the local TFR from Pierce \& Tully (1992, PT92, solid line). The low quality data fit features a shallower slope, possibly due to underestimated $V_{\max }$ values for a fraction of the slow rotators. For matters of clarity, individual error bars are not displayed.

universe and intermediate redshift. To illustrate this, straightforward bisector fits have been applied separately to the high and low quality TF objects, see Fig. 6.4.

For the former, a slope $a=-4.66$ is found, while the low quality data fit yields $a=-3.9$. Since the corresponding RCs only show a weak coverage of the "flat region" at large radii, the $V_{\max }$ values of slowly rotating (low-mass) spirals may be underestimated. Remember that the RCs of local, low-mass spirals often do not show a flat part but merely a slight monotonic increase of $V_{\text {rot }}$ beyond the turnover radius (see chapter 5.2.2 and section 6.4.5). To gain conservative results from the $\mathrm{TF}$ analysis, its main parts will therefore be focussed on the objects with high quality curves.

In Fig. 6.5, the TF offsets are plotted against 


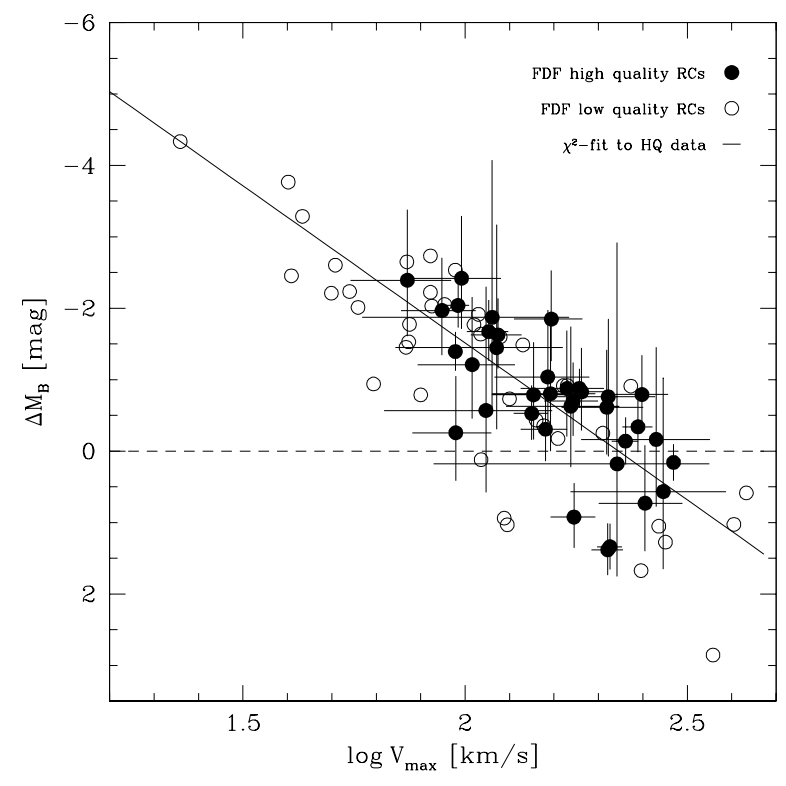

Figure 6.5: Offsets of the FORS Deep Field sample from the local TFR as given by Pierce \& Tully (1992) as a function of maximum rotation velocity. For objects with slow rotation (i.e., low mass), large overluminosities are observed, whereas the fast rotators (high-mass spirals) show similar luminosities as their local counterparts. The dashed line corresponds to a zero evolution, the solid line denotes the $\chi^{2}$-fit to the high quality data.

$V_{\max }$. A linear $\chi^{2}$-fit to the high quality subsample with an error estimate as defined in Eq. 6.10 yields

$\Delta M_{B}=(4.40 \pm 0.69) \log V_{\max }-(10.31 \pm 1.55)$,

corresponding to a brightening by more than two magnitudes for the least massive spirals in the FDF sample and negligible offsets at $V_{\max } \approx 220 \mathrm{~km} / \mathrm{s}$, which on the basis of Eq. 6.5 is the typical rotation for local spirals of luminosity $L_{B}=2 \ldots 3 L_{B}^{*}$.

A $V_{\max }$-dependence of the TF residuals would offer a further explanation for the large scatter $\sigma_{\Delta M}$ addressed above. Since the distant TFR does not show a simple offset with respect to the local sample but a flatter tilt, the scatter is overestimated when derived under the assumption of a constant slope. On the other hand, if the bisector fit to the high quality sample is adopted, the scatter of the residuals of the distant TFR

$$
M_{B}=-4.66 \log V_{\max }-10.43
$$

is $\sigma_{B}=0.85^{m}$ for the complete sample and $\sigma_{B}=0.71^{m}$ for the 36 high quality objects. Since this is still $\sim 1.7$ times the local $B$-band TFR scatter, the spread of the star formation efficiencies indeed probably is enlarged at intermediate redshifts.

To compute the significance of the indicated TFR slope decrease, a bootstrap bisector fit (e.g., Ziegler et al. 2001) was performed, i.e., 100 iterations of repeated bisector fits with random rejection of objects between each iteration. In this approach, the error can be derived from the scatter of the individual fits' slopes. Based on the high quality FDF data, the TFR at $z \approx 0.5$ is

$$
M_{B}=-(4.66 \pm 0.67) \log V_{\max }-(10.43 \pm 1.50),
$$

hence the result is significant on the $4 \sigma$ level. To verify this with a different fitting method, the errors of the absolute magnitudes in the TF diagram were estimated to be

$$
\sigma_{\mathrm{TFR}}^{2}=\sigma_{M B}^{2}+7.48^{2} \sigma_{\operatorname{logv}}^{2} .
$$

The first term on the right hand side corresponds to Eq. 4.32 and the second term is the propagated error of $V_{\max }$ based on the PT92 slope. A linear $\chi^{2}$-fit to the high quality FDF then gives

$$
M_{B}=-(3.55 \pm 0.41) \log V_{\max }-(12.84 \pm 0.92) .
$$

In this approach, the slope change hence is even significant on the $9 \sigma$ level. Note that PT92 do not give an error estimate on the slope, cf. section 6.4.6, where the error on the local slope is taken into account with a different data set.

One further conclusion from the indicated slope change is that the amount of luminosity evolution as a function of redshift given in Eq. 6.9 has 
to be considered a lower limit: towards higher redshifts, only massive spirals meet the selection criterion on the apparent magnitudes of $R \leq 23^{m}$. In turn, only spirals which feature a mild or even negligible evolution in $M_{B}$ are comprised by the FDF sample. Two additional factors that come into play will be addressed in section 6.7. Prior to an extensive discussion of the distant TFR, a second important scaling relation will be presented in the following section.

\subsection{The Distant Velocity-Size Relation}

Via the velocity-size relation (VSR hereafter), the maximum rotation velocity $V_{\max }$ is correlated to the disk scale length $r_{\mathrm{d}}$ : faster rotators feature larger disks. Similar to the analysing power of the TFR, the VSR can be used to quantify a potential evolution of the disk sizes over cosmic time. In the hierarchical merging scenario, disks should be on the average smaller for distant spirals than in the local universe. More precisely, at a given $V_{\max }$, the disk sizes are expected to decrease with increasing redshift.

Mo, Mao \& White (1998, MMW98 hereafter) predict an evolution

$$
r_{d}(z)=r_{d}(0) \times\left[\Omega_{\Lambda}+\Omega_{m}(1+z)^{3}\right]^{-1 / 2}
$$

at fixed $V_{\max }$ for a flat cosmology. Note that this equation only is valid for a constant halo spin parameter $\lambda_{h}$, which is a reasonable assumption for disks in centrifugal equilibrium (ibid.).

As a local reference, the Hay99 sample (introduced in section 6.1) will be used. The publicly available data table gives the isophotal radii at an $I$-band surface brightness of $\mu_{I}=23.5^{m}$. If one assumes a mean central surface brightness of $\mu_{I}=19.4^{m}$ (Hay99), these radii $r_{23.5}$ can be transformed into exponential disk scale lengths via

$$
r_{\mathrm{d}}=\frac{\mathrm{e} \cdot r_{23.5}}{2.5(23.5-19.4)}=\frac{r_{23.5}}{3.771} .
$$

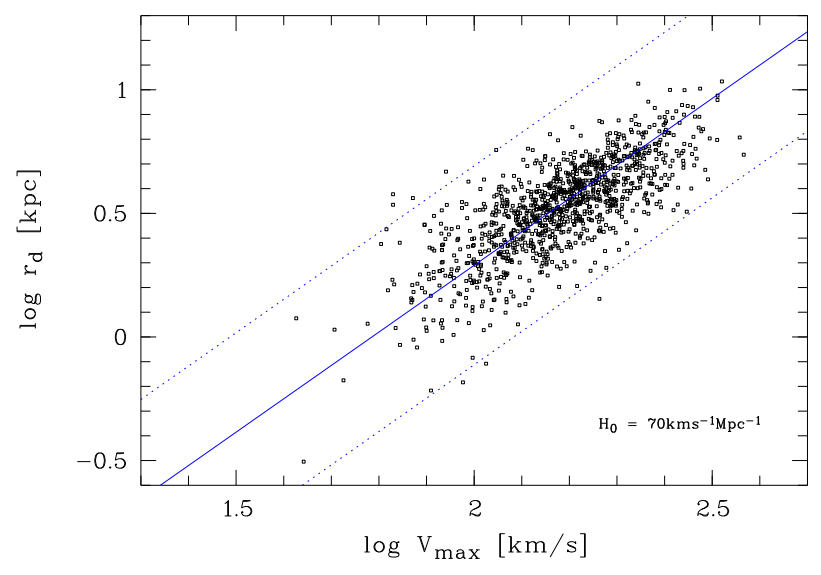

Figure 6.6: The local velocity-size relation of the Haynes et al. (1999) sample. A bisector fit to the 1097 galaxies is denoted by the solid line, the dotted lines give the $3 \sigma$ scatter.

The local VSR of the Hay99 sample is shown in Fig. 6.6. A bisector fit (note that a $\chi^{2}$-fit could not be applied since the individual errors were not available) yields

$$
\log r_{\mathrm{d}}=1.35 \log V_{\max }-2.41
$$

with a scatter of $\sigma_{\text {logrd }}=0.13$.

The distant FDF spirals are compared to this relation in Fig. 6.7. Analogous to the TF offsets, the offsets of the FDF spirals were computed as

$$
\Delta \log r_{\mathrm{d}}=-1.35 \log V_{\max }+2.41+\log r_{\mathrm{d}},
$$

yielding a scatter $\sigma_{\Delta \operatorname{logrd}}=0.26$. Though this is two times the local scatter and thus similar to the discrepancy between local and distant TFR scatter, the distribution of the high quality objects in $\log V_{\max } / \log r_{\mathrm{d}}$-space is so uniform that a free fit is unlikely to be reliable. Possibly the limitations of the ground-based FDF imaging are a key factor here.

As a test, a bootstrap bisector fit was performed. For the high quality data, this yields

$$
\log r_{\mathrm{d}}=(0.77 \pm 0.69) \log V_{\max }-(1.21 \pm 1.53) .
$$

The large error on the slope quantitatively confirms that the quality of the FDF VS diagram is 


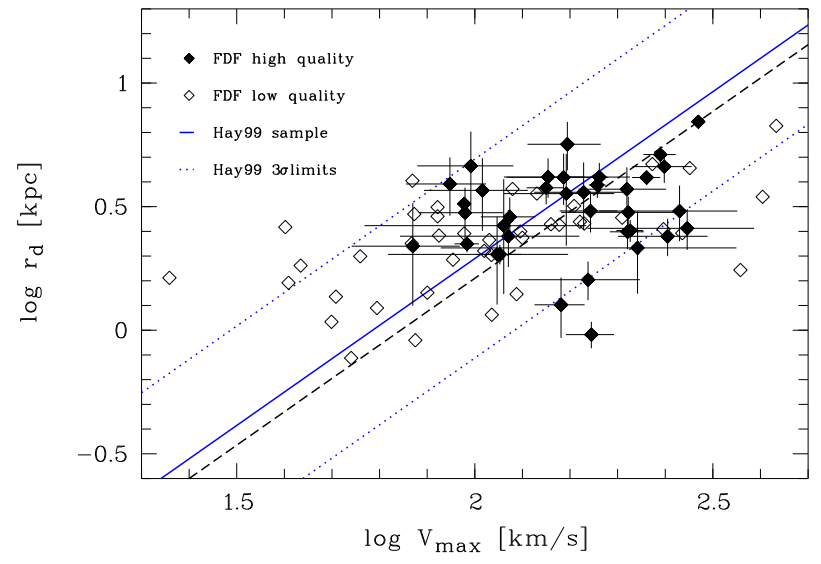

Figure 6.7: Comparison between the FDF spirals and the local velocity-size relation of the Haynes et al. (1999, Hay99) sample. The dashed line denotes assuming a constant local slope - the slightly smaller average disk scale lengths at $z \approx 0.5$, corresponding to an offset $\Delta \log r_{\mathrm{d}}=-0.08$ for the high quality sub-sample.

insufficient for a free two-parameter fit. Moreover, the slope value is in agreement with the local sample within the given errors. It will therefore be assumed in the following that the VSR slope is constant over the redshift range $0<z<1.0$.

The VS offsets indicate a very small decrease of the average distant disk sizes with respect to the local universe, the repective mean and median values are $\overline{\Delta \log r_{\mathrm{d}}}=-0.08$ and $\left\langle\Delta \log r_{\mathrm{d}}\right\rangle=-0.05$ for the high quality objects. Taking into account the scatter $\sigma_{\Delta \operatorname{logrd}}$, these values are consistent with a zero disk size evolution. However, at the average redshift of the FDF spirals, the disk size decrease predicted by simulations is also small: at $z \approx 0.5$, a size evolution $\Delta \log r_{\mathrm{d}}=-0.10$ is expected according to MMW98 (Eq. 6.18).

To perform a test of a possible evolution with redshift, the errors on $\Delta \log r_{\mathrm{d}}$ were calculated as

$$
\sigma_{\Delta \mathrm{VS}}^{2}=\sigma_{\operatorname{logrd}}^{2}+1.35^{2} \sigma_{\log \mathrm{v}}^{2},
$$

where the first term on the right hand side represents the error on the logarithmic scale length

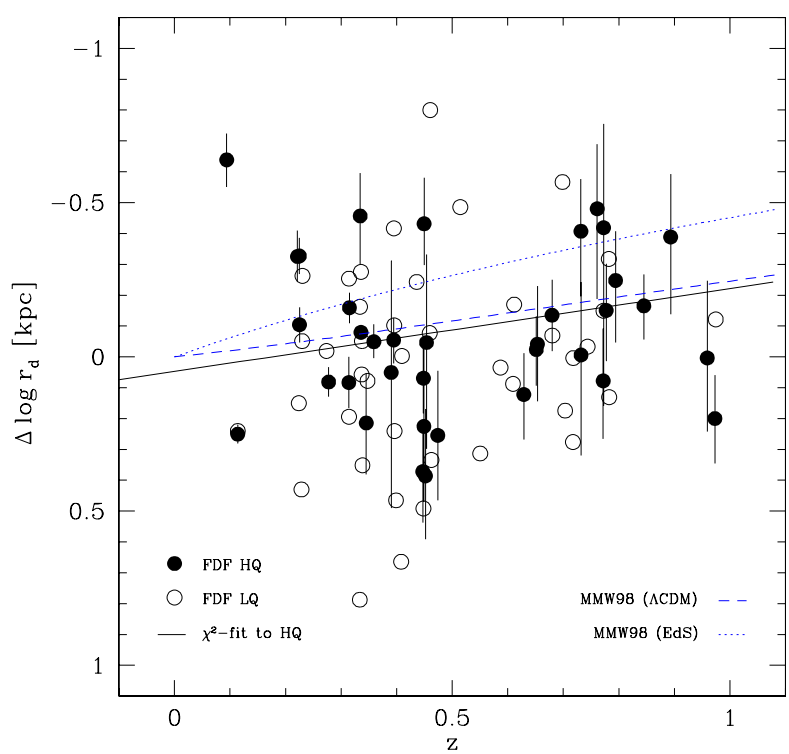

Figure 6.8: Offsets of the FORS Deep Field sample from the local velocity-size relation of the Haynes et al. (1999) sample, plotted against redshift. The FDF sample is sub-divided according to a high (HQ) or low quality (LQ) of the corresponding rotation curves. The solid line gives a $\chi^{2}$-fit to the HQ data, the dashed and dotted lines denote the predictions of simulations by Mo, Mao \& White (1998, MMW98) within the hierarchical merging scenario for a $\Lambda \mathrm{CDM}$ and an Einstein-de Sitter cosmology, respectively. See text for details.

and the second term is the logarithmic propagated error in $V_{\max }$, assuming the local VSR slope. A $\chi^{2}$-fit then yields

$$
\Delta \log r_{\mathrm{d}}=-(0.27 \pm 0.10) \cdot z+(0.05 \pm 0.04)
$$

This fit implies a decrease of the scale length with redshift for a given $V_{\max }$ value, corresponding to disks at $z=1$ which are smaller by a factor of 1.66 than in the local universe.

In Fig. 6.8, the VS offsets are plotted against redshift. The predictions from MMW98 (Eq. 6.18) for the disk size reduction in a hierarchical universe are shown for two flat but fundamentally different cosmologies, namely the concordance cosmology with $\Omega_{\Lambda}=0.7$ and $\Omega_{m}=0.3$ 
(dashed line) and an Einstein-de Sitter cosmos with $\Omega_{\Lambda}=0.0$ and $\Omega_{m}=1.0$ (dotted line). The FDF data are in good agreement with the concordance cosmology, whereas in a flat and purely matter-dominated universe, the disk sizes would have to be $\sim 1.7$ times smaller at $z=1$ than observed.

It can be concluded from this part of the analysis, that the VSR of the FDF sample is less powerful than the TFR. Nevertheless, the slight redshift dependence of the disk sizes is in compliance with theoretical predictions for a $\Lambda \mathrm{CDM}$ hierarchical universe and will be further addressed in the discussion in section 6.4.

Since the distant spiral sample shows evidence for an evolution in the luminosities and the disk sizes, the Freeman relation between these two quantities will not be considered here. Any discrepancy between the local and distant relation in $M_{B} / r_{\mathrm{d}}$-space would introduce a degeneracy in luminosity and size. Hence, no additional information could be gained from an analysis of this scaling relation.

\subsection{Discussion}

Before interpreting the possible physical implications of the correlation between the TF offsets and $V_{\max }$ denoted by Eq. 6.13, a variety of tests will be described which were performed to varify this finding.

In the next section, the potential effect of sample incompleteness on the TF analysis will be analysed, followed by the consideration of potential biases due to galaxy-galaxy interactions or strong star formation. Then, the impact of various intrinsic rotation curve shapes, the question of different conventions for the intrinsic absorption correction and the reliability of the ground-based imaging for a TF analysis will be addressed. A comparison to previous distant TFR studies is given in section 6.5 and it will be shown therein that the TFR slope decrease offers a rather simple explanation for the discrepancies

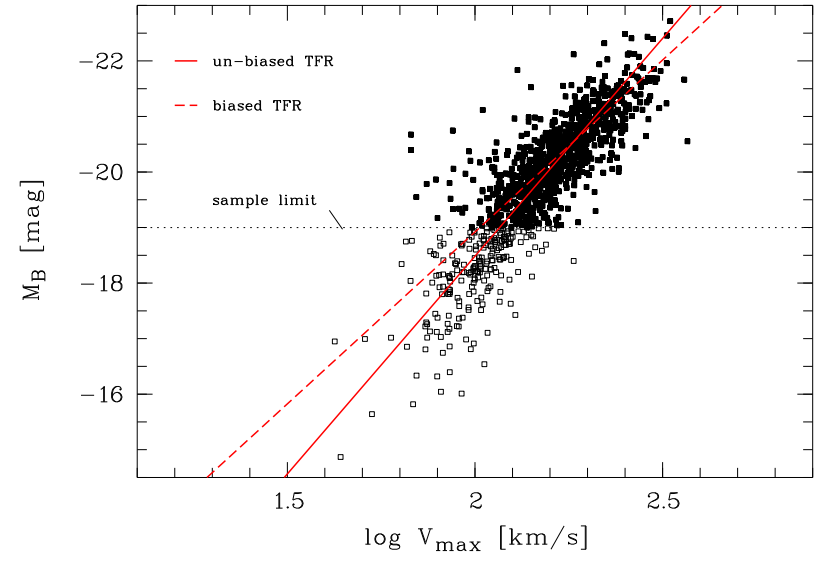

Figure 6.9: Illustration of the incompleteness bias. The dotted line represents a sharp sample limit corresponding to $M_{B}<-19$. An observed sample comprising only galaxies brighter than this limit (filled symbols) would yield a biased TFR slope that is shallower (dashed line) than the un-biased slope (solid line) of the total galaxy population (filled and open symbols). See text for details.

between the findings of different authors in the literature. Finally, the results of this thesis will be discussed in section 6.7 .

\subsubsection{Test of Incompleteness}

One effect that has to be considered in the TF analysis of a data set is the sample incompleteness. As shown in section 4.7, the selection limit on the FDF spirals' apparent magnitudes of $R \leq 23^{m}$ corresponds to a lower luminosity limit that increases with redshift. Any spiral sample that is magnitude-limited does not cover the complete luminosity function (LF) but, towards lower luminosities, only contains a decreasing fraction of the total population.

Fig. 6.9 illustrates this effect for the simplified case of a sharp "cutoff" in luminosity: the TFR which would be derived from an observed sample with spirals brighter than $M_{B}=-19 \mathrm{mag}$ has a shallower slope than the TFR which is valid for the total population of spiral galaxies (which in this figure is denoted by the local Hay99 sample). In other words, the incom- 
pleteness of an observed sample towards low luminosities can introduce an underestimation of the TFR slope. It therefore has to be verified whether this so-called incompleteness bias has an impact on the FDF sample which shows evidence for a decreased TFR slope at $z \approx 0.5$.

Usually, an observed luminosity distribution does not comprise a sharp cutoff in absolute magnitude at the faint end, but a decreasing number of objects per magnitude bin (cf. chapter 4.7). Giovanelli et al. (1997, Gio97 hereafter) introduced a methodology to derive the impact of the incompleteness bias on the TF analysis for a given sample that is defined by its observed LF and $V_{\max }$ distribution. Only the basics of this approach will be described here, for an extensive discription and a test of its reliability see Gio97. The idea of the Gio97 approach is to parameterise the sample incompleteness at the faint end of the LF with a so-called completeness function. This function then is used to simulate the TFR of a spiral sample with observed $V_{\max }$ values on the basis of an un-biased TFR (UTFR). A comparison between the assumed UTFR and the simulated TFR yields the amount of the incompleteness bias, which increases towards slower rotators since these feature, in general, lower luminosities and hence are stronger affected by the sample incompleteness. Four parameters therefore have to be defined in advance of the debiasing:

- UTFR slope

- UTFR zero point

- UTFR scatter

- un-biased LF.

The first two points are a fundamental shortcoming of the Gio97 method (which nevertheless is, to the best of the author's knowledge, the only method in the literature that addresses the incompleteness bias in TF applications): the slope and zero point, which in principle are to be derived via the de-biasing, have to be defined $a$ priori. This is a circular reasoning. It is therefore questionable whether the Gio97 approach is reliable for the derivation of the un-biased TFR. Nevertheless, for testing purposes, this method is very useful here, since it can be quantitatively verified whether the decreased slope of the distant TFR may be induced by the incompleteness bias. To do so, it will therefore be assumed in this section that the TFR slope is constant in the regime $0<z<1$. More precisely, a slightly steeper TFR than yielded by the Hay99 sample was defined as the UTFR:

$$
M_{B}=-8.00 \log V_{\max }-2.56
$$

with an intrinsic scatter 1.5 times larger than the local value. The respective values of slope and scatter were chosen to gain a conservative estimate on the possible bias. For the LF, a Schechter function with an exponent $\alpha=-0.85$ was assumed. Gio97 alternatively used a value $\alpha=-0.5$, which yields a smaller incompleteness bias. Since it is the aim of this test to derive an upper limit on the bias, only the steeper LF with $\alpha=-0.85$ will be considered.

In the first step of the de-basing procedure, the observed LF was divided by the Schechter function, resulting in a completeness histogram $h$. The characteristic luminosity $L^{*}$ in the Schechter function was determined from the bright end of the observed LFs and corresponded to $M_{B}^{*}=-21.3 \mathrm{mag}$ for the FDF spirals and $M_{B}^{*}=-21 \mathrm{mag}$ for the local Hay99 data. The latter has been used for comparison here since the luminosities of the complete PT92 sample were not available.

The completeness histogram then was fitted with the so-called completeness function

$$
c(y)=\frac{1}{\mathrm{e}^{\left(y-y_{f}\right) / \eta}+1} .
$$

Here, $y$ is the absolute $B$ magnitude, $y_{f}$ is the value of $M_{B}$ corresponding to a sample completeness of $50 \%$ and $\eta$ is a tuning factor for the gradient of the completeness function in the regime of incompleteness. 

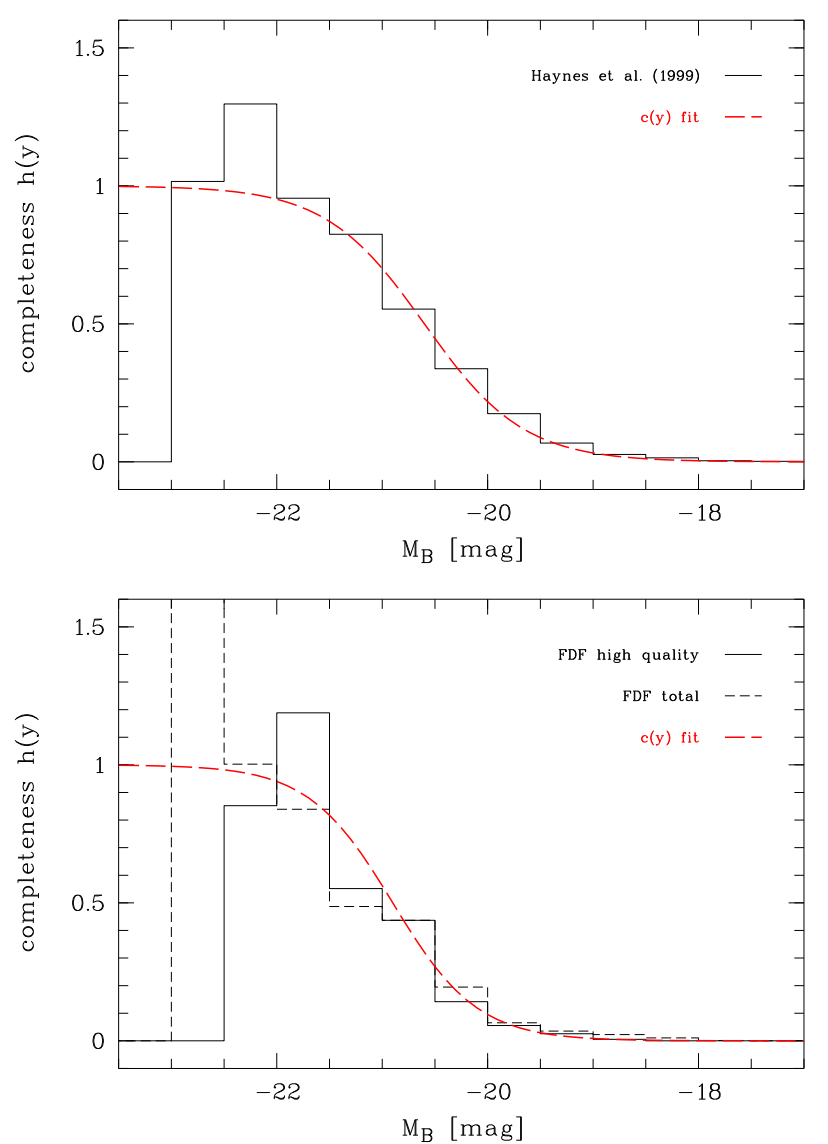

Figure 6.10: Completeness histograms $h(y)$, i.e., the observed luminosity functions divided by the Schechter function, and the corresponding fits with the completeness function $c(y)$ as introduced by Giovanelli (1997). Upper figure: local sample from Haynes et al. (1999), lower figure: FDF high quality sub-sample and total sample. See text for details.

In Fig. 6.10, the completeness histograms $h(y)$ and functions $c(y)$ are shown for the Hay99 and FDF samples, respectively. A completeness of $h(y)=1$ indicates that all galaxies of absolute magnitude $y$ within the probed volume are included in the sample, whereas at $h(y)=0$, no galaxy of absolute magnitude $y$ is comprised by the data. The relatively small number of FDF galaxies complicated the completeness function fit, in particular at the bright end of the observed distribution, which is slightly flatter than expected from the Schechter function. In effect, some magnitude bins feature values $h(y)>1$ (as is the case at $M_{B} \approx-22.25$ in the Hay99 distribution). Note that $h(y)$ has smaller values for the complete FDF sample than for the high quality sub-sample at $M_{B} \approx-21.5 \mathrm{mag}$ since the Schechter function was normalised to the total number of objects at the bright end of the corresponding observed LFs. The parameters of the completeness function fit $c(y)$ were $y_{f}=-20.9$, $\eta=0.4$ for the FDF data and $y_{f}=-20.6$, $\eta=0.47$ for the local Hay99 sample, respectively. Finally, values for $M_{B}$ were simulated on the basis of the observed $V_{\max }$ values, the assumed scatter and the UTFR. Here, the completeness function was used to pass each object through a "completeness filter" which yields the probability for an object with simulated absolute magnitude $y$ to be included in the simulated observed sample. This was done by generating a random number $0<n_{\text {ran }}<1$ and rejecting the object if $n_{\text {ran }}>c(y)$. For a sufficiently large number $n_{\text {it }}$ of iterations per object, this approach allows to estimate the incompleteness bias $m_{\text {bias }}$ as a function of $V_{\max }$ via

$$
m_{\text {bias }}=-a_{\mathrm{u}} \log V_{\text {max }}-b_{\mathrm{u}}+\frac{1}{n_{\mathrm{it}}} \sum_{\nu=1}^{n_{\mathrm{it}}} y_{\nu},
$$

where $a_{\mathrm{u}}$ and $b_{\mathrm{u}}$ are the UTFR slope and zero point, $V_{\max }$ is the observed maximum rotation velocity and $y_{\nu}$ is the simulated absolute magnitude from iteration $\nu$.

The incompleteness biases of the FDF and Hay99 samples are shown as a function of $V_{\max }$ in Fig. 6.11, along with second degree polynomial fits. The FDF sample is affected stronger by the bias than the Hay99 data. To test the impact of these biases, the absolute magnitudes were corrected according to

$$
M_{B \mathrm{n}}=M_{B}-m_{\mathrm{bias}} .
$$

These de-biased values $M_{B \mathrm{n}}$ correspond to lower luminosities, in particular for slow rotators, hence the TFRs should have steeper slopes on the basis of the $M_{B \mathrm{n}}$ values than initially. A 100 

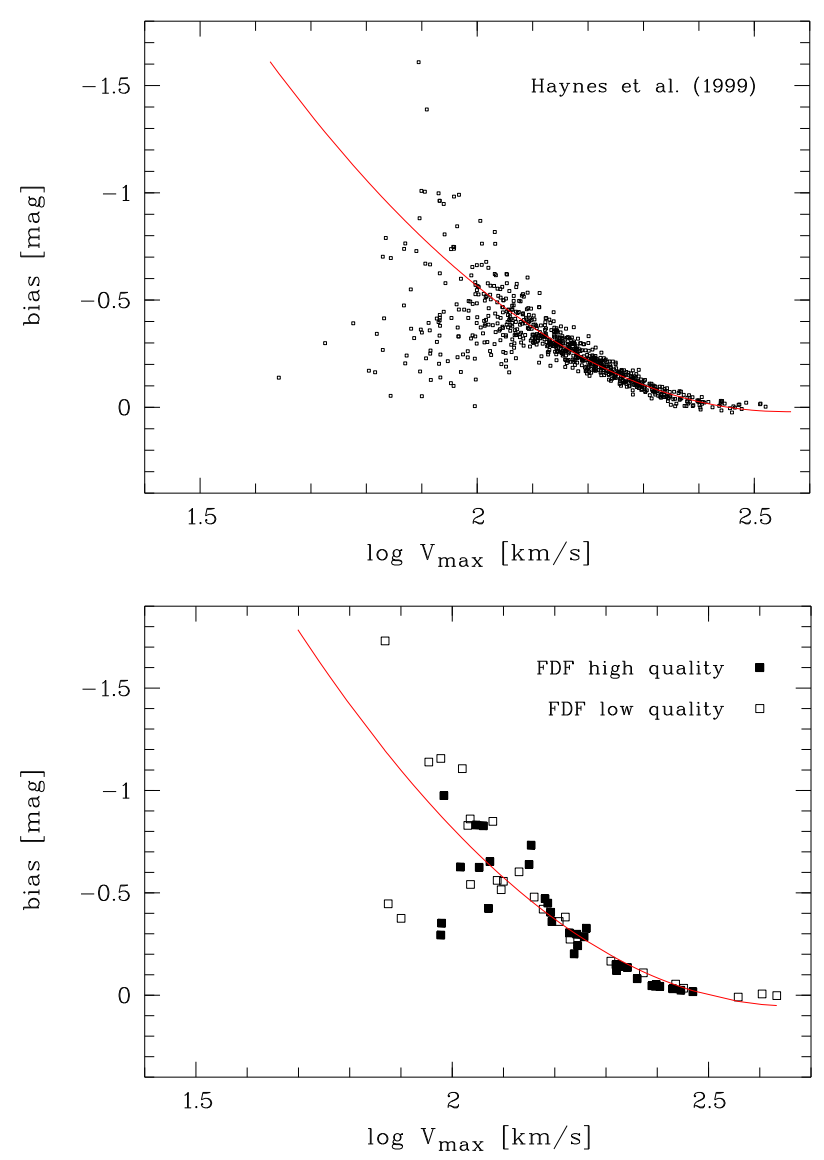

Figure 6.11: Incompleteness biases following Giovanelli et al. (1997), shown as a function of maximum rotation velocity. Upper figure: local sample from Haynes et al. (1999), lower figure: FDF high and low quality sub-samples, respectively. The solid lines denote polynomial fits of second degree. Note that some objects at low $V_{\max }$ values were rejected during the bias computation since they did not pass the incompleteness filter in any of the $n_{\text {it }}$ iterations. In effect, the number of data points in both figures is smaller than the total number of objects in the respective samples, see text for details.

iteration bootstrap bisector fit to the FDF high quality data yields

$$
M_{B \mathrm{n}}=-(6.41 \pm 0.66) \log V_{\max }-(6.18 \pm 1.47),
$$

while the fit to the Hay99 sample is

$$
M_{B \mathrm{n}}=-(9.21 \pm 0.17) \log V_{\max }+(0.48 \pm 0.38) \text {, }
$$

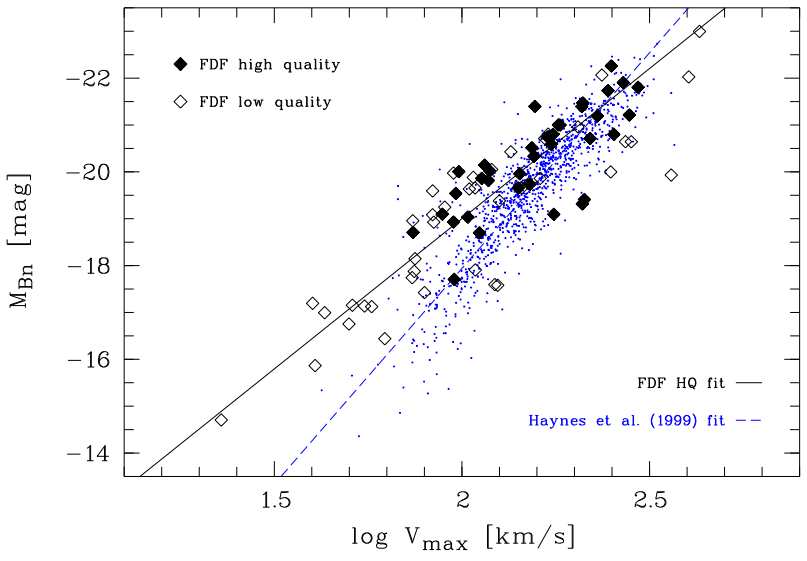

Figure 6.12: Tully-Fisher diagram corrected for the incompleteness bias. Small symbols denote the local Haynes et al. (1999) sample, large symbols represent the distant FDF sample. The solid and dashed lines are bisector fits to the de-biased distant and local samples, respectively. The bias correction does not affect the basic result: a decreased slope of the distant TFR with respect to the local relation.

see also Fig. 6.12.

Possibly, the latter fit indicates a further shortcoming of the Gio97 methodology: the de-biased slope is significantly steeper than the assumed UTFR value of $a_{\mathrm{u}}=-8$, though not the increased but initial intrinsic scatter has been used.

On the other hand, this has no impact on the interpretation of the FDF fit. The de-biased distant TFR is flatter than the UTFR with $>2 \sigma$ confidence or over $95 \%$ probability. If, for the sake of consistency, the de-biased FDF data are compared to the de-biased Hay99 sample, the significance of the slope decrease is $>3 \sigma$, corresponding to $>99 \%$ probability. It has to be concluded that the flatter tilt of the TFR at intermediate redshift cannot be explained as an effect of sample incompleteness only.

However, to avoid the indicated shortcomings of the de-biasing procedure, the further analysis will be performed on the basis of the initial absolute magnitudes. 


\subsubsection{An Effect of the Color-Residual Relation?}

To some extent, a correlation between rotation velocity and $\mathrm{TF}$ offsets is to be expected from previous studies. E.g., Kannappan et al. (2002b) found a color-residual relation (CRR hereafter) that reflects overluminosities of blue spirals and argued that this could be attributed to enhanced star formation. Since spirals with blue colors (late types) feature on the mean lower values of $V_{\max }$ than red, early-type spirals (see section 6.7), a correlation between colors and $\mathrm{TF}$ offsets should coincide with a relation between the offsets and $V_{\max }$. Furthermore, an evolution of the CCR with redshift may induce a change of the TFR slope. This will now be tested.

For local galaxies at $z<0.05$, observed colors deviate only slightly from rest-frame colors. E.g., the observed index $(B-R)$ of type Sc increases by only $0.15^{m}$ between $z=0$ and $z=0.05$. At $z=0.5$, on the other hand, the deviation amounts to $\sim 1 \mathrm{mag}$ (colors derived from synthetic photometry as described in chapter 4.6.4). Therefore, to compare the correlation between colors and TF offsets for the FDF spirals to the local CCR, their $B-R$ color indices had to be computed in rest-frame.

In contrast to the initial derivation of the absolute magnitudes, aperture magnitudes were used for this instead of total magnitudes. These were measured within apertures of two arcseconds diameter on coadded FDF frames that were convolved to the same seeing (see Heidt et al. 2003 for details). Total apparent magnitudes are not reliable to compute color indices since the apparent diameters of the objects could deviate between different passbands.

Similarly to the computation of the absolute $B$ band magnitudes, the aperture magnitudes were taken from different filters, depending on the redshifts. For FDF objects at $z<0.25$, the observed $R_{\text {fors }}$ brightness was transformed into rest-frame Cousins $R_{C}$, while the transformation $I_{\text {fors }} \rightarrow R_{\mathrm{C}}^{\text {rest }}$ was used for all other spirals.
The absorption coefficients with respect to restframe $R_{C}$ were computed following Cardelli et al. (1989).

For the purpose of the CCR, the FDF data were compared to the local sample from Verheijen (2001, Ver01 hereafter). Neither the PT92 sample (data table only available for the 15 "calibrators") nor the Hay99 sample (only I magnitues available) could be used as a local CCR reference.

Since the Ver01 sample was corrected for intrinsic absorption following Tully et al. (1998), the absolute $B$-band magnitudes of the FDF spirals were re-computed in this convention. These are labelled $M_{B 2}$ in the following. The offsets from the local TFR by Ver01 were calculated via

$$
\Delta M_{B 2}=8.1 \log V_{\max }+2.07+M_{B 2} .
$$

Note that Ver01 derived two versions of the TFR with slightly different slopes, based on radio data which covered very large radii. For the first version, the characteristic rotation velocity $V_{\max }$ was derived from the extremum of $V_{\text {rot }}$. This is similar to the UTF convention used here. The second version relied on the rotation velocity $V_{\text {flat }}$ at the farthest covered radii. For spirals of low luminosity, Ver01 found that $V_{\max }$ was slightly smaller than $V_{\text {flat }}$, while for very luminous objects, he found $V_{\text {flat }}<V_{\max }$. This is consistent with the Universal Rotation Curve parameterisation from Persic et al. (1996, see chapter 5.2.2). The TFR slopes derived by Ver01 using $V_{\max }$ and $V_{\text {flat }}$ were -8.1 and -8.7 , respectively. Here, the former value has been adopted to achieve consistency. The question whether a variation of the intrinsic $R C$ shape has an impact on the TF analysis will be the topic of section 6.4.6.

The residuals $\Delta M_{B \text { ver }}$ of the Ver01 relation were derived on the basis of the available absolute magnitudes and $V_{\max }$ values analogous to Eq. 6.31. Using the errors and the absolute $R$ band magnitudes given by Ver01, a linear $\chi^{2}$-fit 


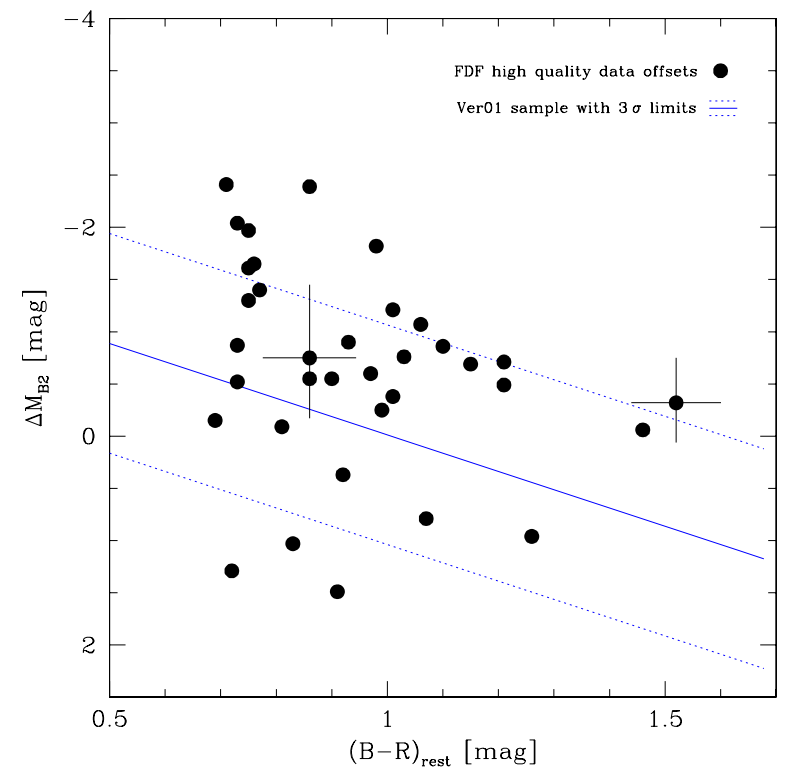

Figure 6.13: Offsets of the high quality FORS Deep Field sample from the local TFR by Verheijen (2001, Ver01) as a function of rest-frame color, compared to the color-residual relation of the local Ver01 sample (solid line, the dotted lines denote the $3 \sigma$ limits). Typical error bars are shown for two FDF objects.

was performed, yielding a local CCR of

$$
\Delta M_{B}=(1.75 \pm 0.19) \cdot(B-R)-(1.76 \pm 0.21) \text {, }
$$

with a scatter of $0.35^{m}$ in $\Delta M_{B}$. In consistency with the findings of Kannappan et al. (2002b), this relation indicates that late-type spirals feature larger TF residuals than early-type spirals. The TF offsets of the FDF high quality sample computed via Eq. 6.31 are shown in comparison to the local CCR in Fig. 6.13. Since the scatter of $\Delta M_{B 2}$ is very large $\left(0.99^{m}\right)$, no fit will be applied. However, it is clear that the offsets do not follow the local CCR. In contrast to the results for the local spirals, the values of $\Delta M_{B 2}$ almost solely populate the regime of overluminosities with a median of $-0.7^{\mathrm{m}}$ and 10 out of 36 galaxies exceed the $3 \sigma$ limits of the local relation on the "bright" side, whereas only 3 objects are $3 \sigma$ outliers on the "faint" side. Note that the median of $\Delta M_{B 2}$ is in very good agreement

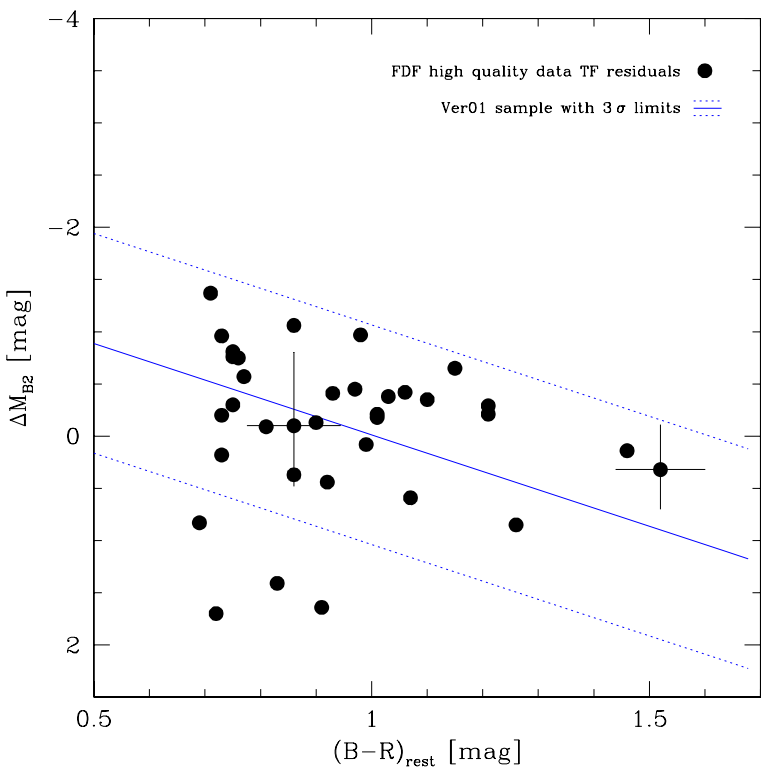

Figure 6.14: Residuals of a free bisector fit to the FDF high quality sample compared to the local colorresidual relation from Verheijen (2001, Ver01). Typical error bars are shown for two FDF objects. A significant evolution of the color-residual relation is not indicated.

with the TF offsets from the PT92 relation which have a median of $-0.77^{m}$. Taking into account the different conventions for the intrinsic absorption correction, these two local samples thus are consistent.

The situation is changed if a variation of the TFR slope and zero point with cosmic time are allowed. On the basis of the high quality data and adopting the Tully et al. (1998) approach for correction of the intrinsic absorption, a bootstrap bisector fit yields

$$
M_{B 2}=-(5.22 \pm 0.69) \log V_{\max }-(9.08 \pm 1.56) .
$$

New residuals using this relation for the FDF sample were derived analogous to Eq. 6.31 and are compared to the local CCR in Fig. 6.14. The scatter of $\Delta M_{B 2}$ is $0.75^{m}$ for the FDF spirals and thus roughly twice as large as the local one. However, the FDF residuals are now mainly distributed within the range $-1 \leq \Delta M_{B} \leq+1$ with 
a median of $-0.21^{m}$. Except for four underluminous outliers (located at relatively low respective redshifts of $0.09,0.22,0.22$ and 0.35 ), the distant spirals are distributed similar to the local CCR. No indications of a steeper CCR at intermediate redshift are found.

It can be concluded that the large $\mathrm{TF}$ offsets according to Eqs. 6.8 or 6.31 can hardly be attributed to an evolution of the CCR. In turn, the indicated TFR slope decrease at intermediate redshift is improbably induced by an evolving color-residual relation.

\subsubsection{Test of Environmental Effects}

Another process that could affect the distant TFR are galaxy-galaxy interactions. According to Kannappan et al. (2002a), the TF offsets of spirals in close pairs can be as large as up to several magnitudes due to an enhanced star formation induced by tidal forces. Since gravitational interactions are likely to have been frequent in earlier cosmic stages of a hierarchical universe and are more effective for low-mass objects (e.g., Lambas et al. 2002), a decreased TFR slope could arise if a significant fraction of the spirals have nearby companions. It will be tested now whether close galaxy encounters are a key factor in the FDF sample.

To perform a search for galaxy pair candidates, the complete set of $113 \mathrm{FDF}$ spirals with derived redshifts was combined with $31 \mathrm{E} / \mathrm{S} 0$ galaxies (observed simultaneously with the spirals, see chapter 2.1) and lower resolution spectra from the FDF high $-z$ campaign (Noll et al. 2003), yielding a total of 267 galaxies at $z<1$. As upper limits on the three-dimensional separation of two pair candidates, the results of Lambas et al. (2002) were adopted. Based on a sample of $\sim 1850$ galaxy pairs from the $2 \mathrm{dF}$ Galaxy Redshift Survey, the authors found that the specific star formation is enhanced for objects with projected distances $D_{\text {proj }} \leq 100 \mathrm{kpc}$ and relative radial velocities $\Delta V_{\text {sys }} \leq 250 \mathrm{~km} / \mathrm{s}$.

12 spirals out of the $77 \mathrm{TF}$ objects show spectro-

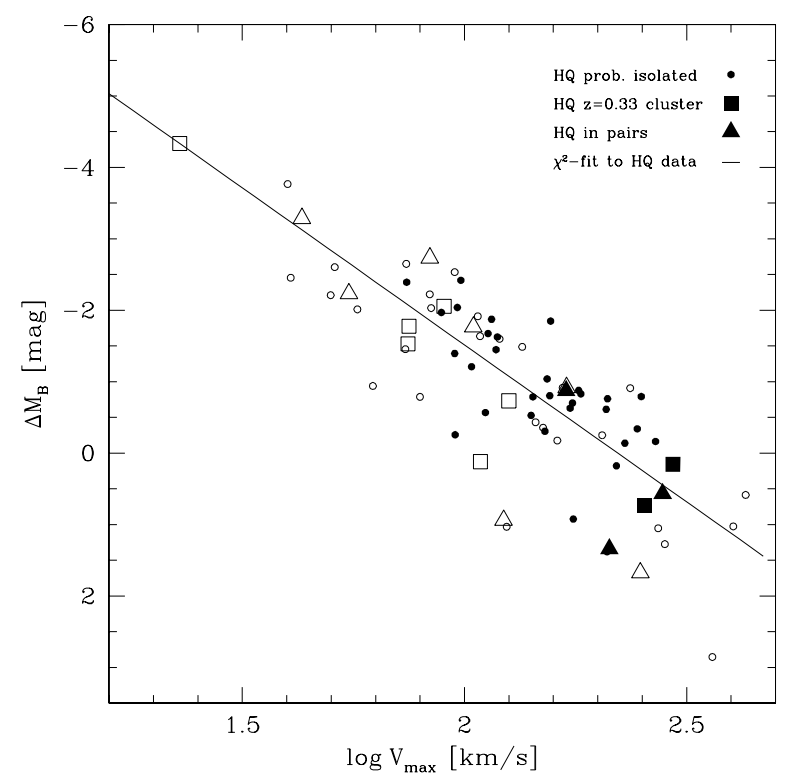

Figure 6.15: Offsets of the FORS Deep Field spirals from the local TFR as given by Pierce \& Tully (1992) as a function of maximum rotation velocity. The FDF sample is sub-divided according to the environment: squares denote potential members of a cluster at $z=0.33$, triangles are objects featuring neighboring galaxies within $\Delta V_{\text {sys }} \leq 250 \mathrm{~km} / \mathrm{s}$ and $D_{\text {proj }} \leq 100 \mathrm{kpc}$ and circles represent probably isolated galaxies. Filled symbols denote spirals with high quality rotation curves.

scopically confirmed neighbors within these limits. Of these galaxies, two are probably members of the cluster at $z=0.33$ located in the southwestern corner of the FDF (see chapter 4.1). The offsets of the pair/cluster candidates from the local PT92 TFR are shown along with the probably isolated objects in Fig. 6.15. The two sub-samples are similarly distributed. This is confirmed by the TF residuals computed according to the bisector fit of the high quality sample (Eq. 6.15); the pair/cluster candidates have a median of $\left\langle\Delta M_{B}\right\rangle=-0.03^{m}$, indicating that they obey the same TFR as the rest of the sample.

As a further test, the 31 high quality spirals without detected companions were fitted with a boot- 
strap bisector fit, resulting in

$$
M_{B}=(-5.01 \pm 0.74) \log V_{\max }-(9.75 \pm 1.61),
$$

which is only very slightly steeper a slope than in the initial fit $(a=-4.66)$ and perfectly consistent within the errors.

A mild overrepresentation of pair/cluster candidates towards low rotation velocities may be deduced from Fig. 6.15. These are all spirals classified as low quality data, i.e., their RCs have a relatively small radial extent and/or show asymmetries. In total, only 5 out of 18 pair/cluster candidates have high quality curves, whereas for the rest of the sample this fraction amounts to $53 \%$. This is in agreement with the results of Kannappan et al. (2002a), who find that galaxies in close pairs frequently show asymmetric or truncated RCs.

A link between RC quality and nearby companions is also implied by Fig. 6.16, where the TF offsets $\Delta M_{B}$ from the TFR by PT92 are shown as a function of projected distance $D_{\text {nn }}$ to the nearest detected neighbor. Note that this diagram includes only 63 FDF spirals, since for 14 objects, no neighbors satisfying $\Delta V_{\text {sys }} \leq 250 \mathrm{~km} / \mathrm{s}$ were found within the field-of-view of FDF.

Several conclusions can be drawn from this graph. Firstly, objects with low quality RCs on the average have a much smaller projected distance $\left(\left\langle D_{\mathrm{nn}}\right\rangle=283 \mathrm{kpc}\right)$ to the nearest detected companion galaxy than the high quality objects $\left(\left(\left\langle D_{\mathrm{nn}}\right\rangle=712 \mathrm{kpc}\right)\right.$. Interestingly, the nonTF objects which have been rejected due to large asymmetries, solid-body rotation or no detectable rotation at all, do not have a smaller average distance $\left(\left\langle D_{\mathrm{nn}}\right\rangle=294 \mathrm{kpc}\right)$ than the low quality data. This may be due to a fraction of objects with kinematic distortions that did not arise from galaxy-galaxy interactions but kinematic imprints of, e.g., a bar or a lopsided DM halo (Jog 1999).

Furthermore, a trend of enlarging TF offsets towards smaller distances is observed for the low

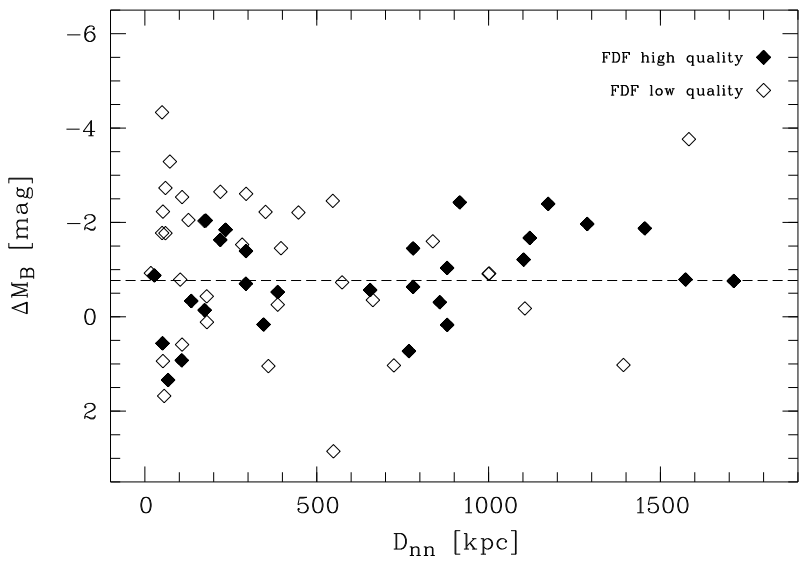

Figure 6.16: Offsets $\Delta M_{B}$ of the FORS Deep Field spirals from the local TFR as given by Pierce \& Tully (1992) as a function of projected distance $D_{\text {nn }}$ to the respective nearest neighbors within $\Delta V_{\text {sys }} \leq 250 \mathrm{~km} / \mathrm{s}$. The dashed line corresponds to the median $\left\langle\Delta M_{B}\right\rangle=-0.77^{m}$ in the high quality sub-sample. The slight increase of the offsets with increasing $D_{\text {nn }}$ confirms that the high quality data can hardly be affected by an enhanced star formation due to tidal interactions.

quality objects. This may be understood as an effect of gravitational interactions which have induced an enhanced star formation and also had a kinematic imprint on the RCs (more precisely, on their quality).

The high quality sample, on the other hand, is very unlikely to be affected by tidal interactions. As is clear from Fig. 6.16, the TF offsets even decrease towards smaller nearest neighbor distances. A triggering of star formation via tidal forces by nearby companions can thus be ruled out for this part of the FDF sample.

The nearest neighbor search cannot yield all pairs at $z<1$ within the FDF, since spectroscopic redshifts are available only for a fraction of the galaxies in this regime (both the TF and the high $-z$ samples have a limit in apparent brightness). Photometric redshifts, on the other hand, are not sufficiently accurate to be used here. According to Gabasch et al. (2003), the typical rel- 
ative error in the final FDF catalogue is

$$
\frac{\sigma_{z p}}{\left(1+z_{p}\right)} \approx 0.03
$$

corresponding to $>10000 \mathrm{~km} / \mathrm{s}$ at $z_{p}=0.5$. This exceeds the adopted upper limit on the relative velocity $\Delta V_{\text {sys }}$ by a factor of 40 .

However, the aim of this section was to clarify whether the candidates which are identified imply a connection between the large $\mathrm{TF}$ offsets of low-mass spirals and close galaxy encounters. From the tests given above it can be concluded that, at least for the high quality data which the major parts of the analysis rely on, a bias towards overluminosities due to gravitational interactions is very unlikely.

\subsubsection{Star Formation Rates}

The previous section confirms that the FDF high quality sample can be considered representative for the field and is most probably not affected by recent galaxy-galaxy interactions. However, a fraction of the spirals could have recently undergone phases of enhanced star formation due to, e.g., the accretion of dwarf galaxies, which could be too faint to be included in the spectroscopic FDF studies and thus would be missed by the near neighbor search. Such accretion events are expected to be a frequent phenomenon towards higher redshifts in a hierarchical universe. To check this, the Star Formation Rates (SFRs) $\psi$ will now be considered. The most robust emission feature for the computation of SFRs, the $\mathrm{H} \alpha$ line, is visible only in four spirals from the FDF sample. However, Kennicutt (1992b) found a correlation between the equivalent widths of $\mathrm{H} \alpha$ and $[\mathrm{O} \mathrm{II}] 3727$ and used it to derive a formula for [O II]-based SFRs:

$\psi=2.7 \cdot 10^{-12} \frac{L_{B}}{L_{B, \odot}} \operatorname{EW}([\mathrm{OII}]) E(\mathrm{H} \alpha)\left[M_{\odot} / \mathrm{yr}\right]$.

Here, $L_{B, \odot}$ is the $B$-band luminosity of the sun (set to a corresponding absolute magnitude

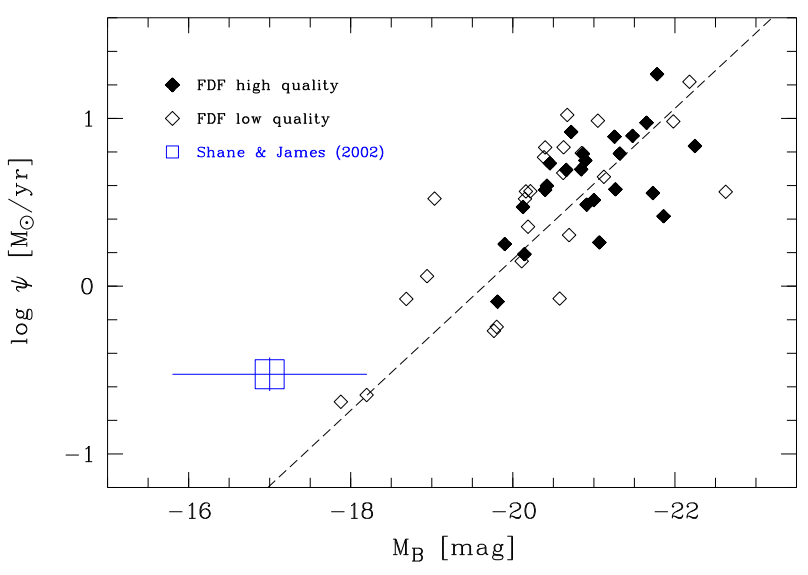

Figure 6.17: Star Formation Rates (SFRs) $\psi$ of the FDF spirals as a function of absolute $B$ magnitude (filled and open lozenges). The open square denotes a local sample including 104 spirals from Shane \& James (2002) with the error bars corresponding to the respective scatters in $\psi$ and $M_{B}$. Note that the SFRs have been computed only for FDF objects with a visible [O II] doublet $\left(N_{\mathrm{obj}}=49\right)$. The dashed line is a bisector fit to all FDF spirals.

$M_{B}=5.48$ following Kennicutt), $\mathrm{EW}([\mathrm{O} \mathrm{II}])$ is the equivalent width of the [O II] doublet in restframe and $E(\mathrm{H} \alpha)$ is a factor that accounts for the difference between the intrinsic absorption at the wavelengths of the $\mathrm{H} \alpha$ line and of the [O II] doublet; Kennicutt gives $E(\mathrm{H} \alpha)=1.0^{m}$ for spirals.

49 spirals from the TF sample have spectra with a visible [O II] doublet. The SFRs of these galaxies cover a range $0.2 M_{\odot} / \mathrm{yr} \leq \psi \leq 18.4 M_{\odot} / \mathrm{yr}$ with a median $\langle\psi\rangle=3.8 M_{\odot} / \mathrm{yr}$; the $24 \mathrm{ob}-$ jects with high quality $\mathrm{RCs}$ fall in the range $0.8 M_{\odot} / \mathrm{yr} \leq \psi \leq 18.4 M_{\odot} / \mathrm{yr}$ and show a slightly larger median of $\langle\psi\rangle=4.5 M_{\odot} / \mathrm{yr}$. These values do not exceed those of normal disks at low redshift; e.g., Kennicutt (1983) gives an upper limit of $20 M_{\odot} / \mathrm{yr}$ for massive spirals. The term "normal" here refers to unperturbed galaxies. Violent interaction events like mergers can induce star formation rates which are much higher, up to $1000 M_{\odot} /$ yr (e.g., Kennicutt 1998).

In Fig. 6.17, the SFRs are shown as a function of $M_{B}$ in comparison to the values of a local spiral 
study comprising 104 galaxies (Shane \& James 2002). Since the data of the individual objects were not available, the mean and scatter in $\psi$ and $M_{B}$ are used to indicate the respective ranges. The distribution of the FDF spirals as well as a bisector fit to the data points (RC quality unconsidered) do not indicate an enhanced SF for the given range in luminosity - the local reference even shows slightly higher average values of $\psi$ with respect to the FDF fit.

The range in $\psi$ of the distant spirals confirms that they are normal star-forming galaxies which do not comprise unusually high SFRs or even indications of starbursts. This finding will be further discussed in section 6.7.

\subsubsection{The Intrinsic RC Shape}

Is it possible that a false assumption about the shape of the intrinsic RC is affecting the TF offsets? As stated in chapter 5.2.5, the characteristic rotation velocities are highly consistent for the "rise-turnover-flat" shape and the Universal Rotation Curve (URC) from Persic \& Salucci (1991), and differ only slightly if the values $V_{\text {opt }}$ according to the parameterisation of the URC given in Persic et al. (1996, URC96) are used. Considering only high quality RCs and the results from the $\chi^{2}$-fits (Eq. 5.17), a negligible difference $(<0.1 \%)$ between $V_{\max \chi}$ and $V_{\text {opt } \chi}$ is found for spirals with $V_{\max \chi}>150 \mathrm{~km} / \mathrm{s}$. For slower rotators, the median deviation amounts to only $4.4 \%$. Bootstrap bisector fits yield respective TFRs of

$M_{B}=(-3.79 \pm 0.67) \log V_{\max \chi}-(12.45 \pm 1.47)$

and

$M_{B}=(-3.57 \pm 0.83) \log V_{\text {opt } \chi}-(12.87 \pm 1.83)$.

Hence, no significant dependence on the intrinsic RC shape is found for the high quality subsample.

The TFR based on $\chi^{2}$-fitted $\mathrm{RC}$ modelling (Eq. 6.37) has a slightly shallower slope than the relation comprising the $V_{\max }$ values fitted by-eye (Eq. 6.15). This strengthens the indications of slightly underestimated maximum rotation velocities from the $\chi^{2}$ approach in the low-mass regime that have been addressed in chapter 5.2.2. All parts of the analysis rely on the visually derived $V_{\max }$ values and thus yield conservative results, in particular for the TF offsets.

\subsubsection{Intrinsic Absorption Correction}

The convention for intrinsic absorption correction that has been adopted for the FDF spirals is purely inclination-dependent, following Tully \& Fouqué (1985, TF85 hereafter). This approach introduces a fixed optical depth and fractional dust disk thickness for the entire sample. As stated in chapter 4.6.5, Tully et al. (1998, Tu98 hereafter) have found evidence for an internal extinction law which also depends on the maximum rotation velocity. The authors derived a higher amount of absorption in fast rotators than for spirals of low $V_{\max }$.

In effect, the TFR slope of a given sample is steeper in the Tu98 convention with respect to the Tu85 method. However, the absorption in distant and local spirals must be calculated consistently. The respective slopes for the local sample from Verheijen (2001) and the FDF high quality data are $-8.1 \pm 0.3$ and $-5.22 \pm 0.67$ (Eq. 6.33) under the assumption of the Tu98 approach. A TFR slope change thus is significant on the $\sim 3 \sigma$ confidence level, in compliance with the result based on the PT92 sample within the Tu85 convention $(\sim 4 \sigma)$. This is strengthened by the agreement between the respective TF offsets, $\left\langle\Delta M_{B}\right\rangle=-0.77^{m}$ with PT92 as the local reference and $\left\langle\Delta M_{B 2}\right\rangle=-0.70^{m}$ based on the Verheijen data.

An impact of the convention for intrinsic absorption correction on the indicated TFR slope decrease hence is not found. For the sake of direct comparability with the previous TFR studies, which all used the method by TF85, the latter has been used as a standard in this thesis. 


\subsubsection{The Influence of Ground-Based Imaging}

As a final test, the findings of the HDF simulation (chapter 4.4) have to be considered. It was found that the one-component surface brightness profile fits to the FDF spirals might lead to underestimated inclinations in case of large intrinsic bulge-to-total ratios. As an indicator for a potential bulge contribution to the profile, the concentration index $C$ can be used. In Fig. 6.18, the TF offsets from the PT92 relation are shown as a function of $V_{\max }$ for the high quality objects, sub-divided according to $\log C$. The spirals with steep light profiles $(\log C>-0.4)$ and thus possibly strong bulge components mainly populate the regime of small offsets, as is to be expected since an early morphological type coincides with an early spectral type (see chapter 4.5). The latter, in turn, typically are fast rotators, hence these objects feature mild TF offsets.

The important point about Fig. 6.18 is that the spirals with early-type morphology are not biased towards the high-velocity side of the $\chi^{2}$ fit to the full high quality sample (Eq. 6.13). Remember that large bulge-to-total ratios were found to coincide with underestimated inclination angles, which in turn lead to overestimated values of $V_{\max }$. This effect is not indicated by the distribution of the spirals with $\log C>-0.4$ and is unlikely to affect the correlation between $\Delta M_{B}$ and $V_{\max }$.

Underestimated inclinations (overestimated $V_{\max }$ values) due to apparently small, unresolved disks are also improbable. Dividing the high quality data into two groups with $r_{\mathrm{d}}>0.54 \operatorname{arcsec}$ and $r_{\mathrm{d}}<0.54$ arcsec, each containing 18 galaxies, it is found that the median TF offsets are very similar; a value of $\left\langle\Delta M_{B}\right\rangle=-0.79^{m}$ is yielded for the former, while the latter have $\left\langle\Delta M_{B}\right\rangle=-0.73^{m}$.

As has been already argued on the basis of the type-independent inclinations, the groundbased imaging of the FDF spirals is confirmed to feature a sufficient spatial resolution for the

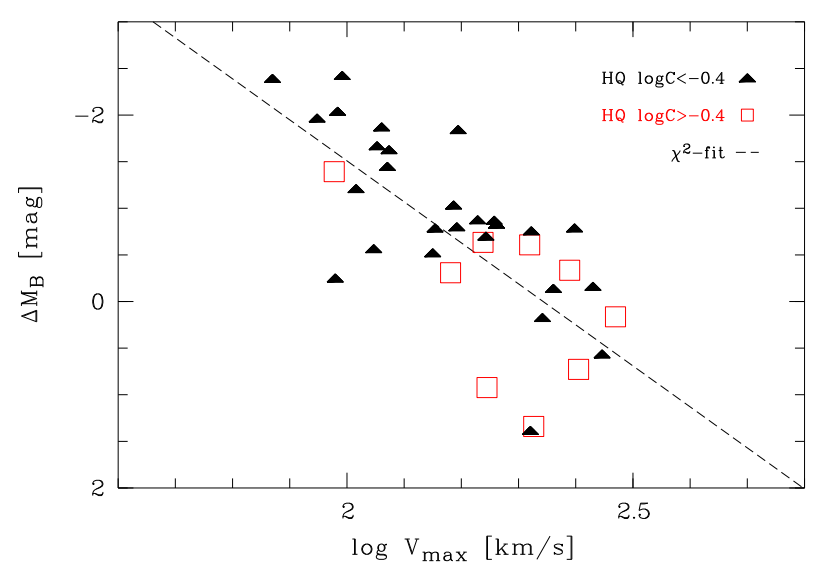

Figure 6.18: TF offsets of the FDF high quality sample from the local TFR by Pierce \& Tully (1992) as function of $V_{\max }$. Filled triangles denote spirals with a logarithmic concentration index $\log C<-0.4$, open squares indicate spirals with $\log C>-0.4$ which possibly have unresolved bulge components. The dashed line represents the $\chi^{2}$-fit to all high quality objects.

purpose of a TF analysis.

\subsection{Previous Studies}

It has been shown in the sections above that the TFR slope decrease indicated by the FDF data is unlikely to reflect some kind of systematic error. In the following, the implications of a correlation between $V_{\max }$ and the amount of evolution in luminosity shall be discussed. To begin with, the discrepancies between many of the previous distant spiral studies (reviewed in chapter 1) will now be compared to the results yielded by the FDF sample.

In chapter 5.3, a connection between the SED type and $V_{\max }$ was found, very similar to characteristics of spirals in the local universe. Blue, late-type spirals are on average slower rotators than red, early-type spirals (e.g., Rubin et al. 1985). Respective median values of $\left\langle V_{\max }\right\rangle \approx 248 \mathrm{~km} / \mathrm{s}$ for types $\mathrm{Sb}$ or earlier, $\left\langle V_{\max }\right\rangle \approx 145 \mathrm{~km} / \mathrm{s}$ for type $\mathrm{Sc}$ and $\left\langle V_{\max }\right\rangle \approx 89 \mathrm{~km} / \mathrm{s}$ for types $\mathrm{Sdm}$ or later were 


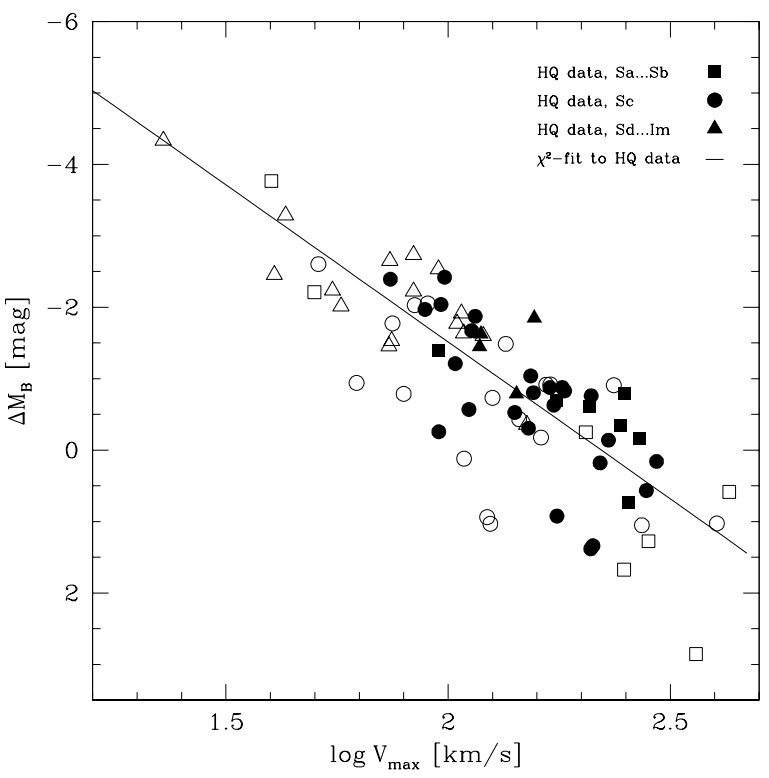

Figure 6.19: Offsets of the FDF sample from the local TFR as given by Pierce \& Tully (1992) as a function of maximum rotation velocity, sub-divided according to SED type into Sb or earlier (squares), $\mathrm{Sc}$ (circles) and Sd or later (triangles), respectively. Filled symbols denote high quality rotation curves, open symbols represent the low quality data.

derived for the TF objects. Since a correlation between the TF offsets and $V_{\max }$ is observed, these $V_{\max }$ distributions imply a dependence of the mean luminosity offsets on SED type as is illustrated in Fig. 6.19.

A consequence of this is a potential selection effect for small samples which mainly comprise a certain sub-type. If a data set was biased towards late-type spirals due to the target selection on blue colors (like, e.g., Simard \& Pritchet 1998) or strong emission lines (e.g., Rix et al. 1997), a considerable evolution in luminosity would be derived. On the other hand, if a data set preferably contains early-type spirals, i.e. large disks (Vogt et al. 1996), only a modest luminosity offset from the local TFR will be observed. The mass-dependent luminosity evolution hence offers a simple explanation for the discrepancies between samples with small number

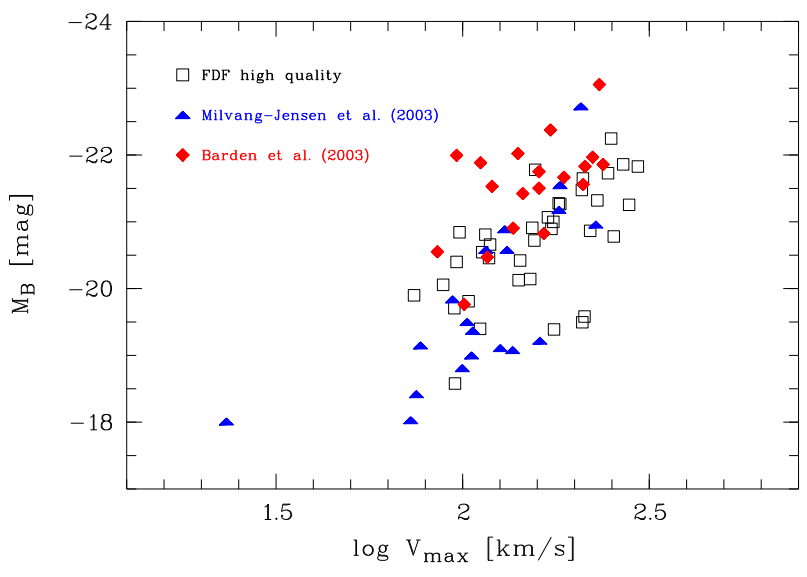

Figure 6.20: FDF high quality data compared to the distant TF samples from Milvang-Jensen et al. (2003) and Barden et al. (2003).

statistics; these samples were too small to test a dependence of the TFR slope on look-back time and also introduced selection effects.

Two distant spiral studies published most recently are Milvang-Jensen et al. (2003, Mil03 hereafter) and Barden et al. (2003, Bar03), both comprising 19 spirals at respective median redshifts $\langle z\rangle=0.37$ and $\langle z\rangle=0.86$. Dr. B. Milvang-Jensen kindly provided the data table on his sample. In Fig. 6.20, these two studies are shown in comparison to the FDF high quality sample. The absolute magnitudes of Mil03 have been re-computed to the concordance cosmology, and the Bar03 values were corrected by $-0.27^{m}$ to be consistent with the faceon Tully \& Fouqué convention for intrinsic absorption adopted here.

While the Mil03 sample falls on the lowluminosity side of the FDF spirals, the objects from Bar03 on the average are more luminous. Just as a test, bootstrap fits were applied to both data sets, yielding a slope $a=-5.57 \pm 1.70$ for Mil03 and $a=-4.83 \pm 1.31$ for Bar03, i.e., some evidence for a slope decrease is indicated by the latter, while the former is almost consistent with the local slope of PT92 within the errors.

The only TF study of distant spirals which, besides this thesis, comprised a sufficiently large 
number of objects to particularly test the evolution of the slope is the sample from the DEEP Groth Strip survey (Vogt 2000) with 100 spirals at $\langle z\rangle \approx 0.5$. As has been previously stated, a constant TFR slope and only a small mean offset of $\Delta M_{B} \approx-0.2^{m}$ are found therein. On the other hand, an analysis of the distant luminosity-metallicity relation, which is based on the same survey (Kobulnicky et al. 2003), favours a combined evolution in luminosity and metallicity that is largest for objects in the regime $M_{B} \approx-19$ and negligible for spirals with $M_{B} \approx-22$. While this finding would be consistent with the results presented in this thesis - the authors determined a value range $\Delta M_{B} \approx-1^{m} \ldots-2^{m}$ for the least luminous galaxies - , the Vogt result utilising the TFR obviously is in contradiction to it. On the other hand, the disk size decrease between $z=0$ and $z=1$ found by $\operatorname{Vogt}\left(\Delta \log r_{\mathrm{d}} \approx-0.2\right)$ is in good agreement with the redshift evolution of the VSR offsets derived in section 6.3.

\subsection{Stellar Population Models}

A direct comparison of the amount of brightening deduced from the TFR to predictions of stellar population models is a complicated matter, since the former reflects several competing effects.

On the one hand, the younger stellar populations at intermediate redshifts coincide with a decrease of the mass-to-light ratio by an increased fraction of hot, blue, high-mass (and therefore short-lived) stars. Additionally, the metallicities may be lower.

On the other hand, since less gas has been consumed via star formation, the gas mass fraction most probably increases with look-back time, thereby increasing the mass-to-light ratio. Moreover, due to the smaller disks for a given maximum rotation velocity, the masses on the mean should be lower towards higher redshifts. In effect, a decrease of the masses and an increase of the gas mass fraction would tend to shift distant spirals to the low-luminosity side of the local TFR, whereas lower stellar mass-to-light ratios and lower metallicities would result in a shift to the high-luminosity side. A domination of the first two of these processes in fast rotators could explain why the most massive FDF spirals are underluminous with respect to the local sample in the TF diagram.

As already stated, the correlation between luminosity evolution and redshift is most probably a lower limit; Eq. 6.9 corresponds to a decrease of $\sim 1.3^{m}$ between $z=1$ and the local universe. To give an example for theoretical predictions, chemically consistent evolutionary synthesis models by Möller et al. (2001) yield an evolution of $\sim 1.5^{m}$ in rest-frame $B$ for an Sc spiral over this redshift range.

\subsection{Further Discussion and Conclusions}

The analysis of the distant FDF spirals has yielded several results which point towards a normal field spiral population and are consistent with other recent observational and theoretical studies.

The basic scheme for present-day galaxies, the Hubble sequence, shows a correlation of spectral type, morphological type and the kinematics. It has been found in the analysis of the FDF spirals that this connection is also valid for galaxies in the redshift range $0.1<z<1.0$, corresponding to a look-back time of more than half the age of the universe. Spirals with later SED types show higher degrees of asymmetry and less concentrated light profiles than spirals of early SED types. This is in compliance with studies in the Hubble Deep Fields which indicated that the evolution of the Hubble sequence took place at redshifts $1<z<2$, and that spirals in the range $0.5<z<1.0$ only are more asymmetric than their counterparts at lower redshifts (e.g., Kajisawa \& Yamada 2001, van den Bergh 2002). 
Moreover, the FDF velocity-size relation showed some evidence for a decrease of the scale lengths with redshift, corresponding to disks at $z=1$ that have only $60 \%$ the size of local disks for a given maximum rotation velocity. This is to be expected for a hierarchical universe and in very good quantitative agreement with the predictions of numerical simulations by, e.g., Mo, Mao \& White (1998) and observations by, e.g., Vogt (2000).

Concerning their kinematical properties, the FDF spirals are similar to local references. The distributions in $V_{\max }$ are consistent, and the distant spirals also show the link between the average $V_{\max }$ and type that is a well known feature of present-day sprials. Roughly half of the FDF spiral rotation curves have a sufficient spatial extent to confirm a flat region at large radii, indicating that the mass distribution is dominated by a Dark Matter halo, analogous to spirals in the local universe.

All these findings are consistent with the paradigm of modern cosmology, the Cold Dark Matter hierarchical universe. But, the TullyFisher relation of the distant spirals implies an evolution which is hard to understand within the framework of this scenario.

The extensive tests described within the previous sections confirmed that the decreased slope of the distant TFR with respect to local data sets cannot be explained in terms of a systematic error or peculiar porperties of the FDF sample. The flatter tilt of the TFR - equivalent to a correlation between the TF offset $\Delta M_{B}$ and $V_{\max }$ - points towards a mass-dependent luminosity evolution that is larger for lower masses.

To confirm this, a linear $\chi^{2}$-fit to the TF offsets as a function of the total masses $M_{\text {vir }}$ has been performed, yielding

$$
\Delta M_{B}=(1.14 \pm 0.27) \log \frac{M_{\text {vir }}}{M_{\odot}}-(13.46 \pm 3.09)
$$

for the 36 spirals with high quality RCs. For this fit, the errors $\sigma_{\text {Mvir }}$ on the total masses
(Eq. 5.23) have been propagated into errors on the offsets and quadratically combined with the errors $\sigma_{\Delta \mathrm{TF}}$ defined in Eq. 6.10.

The ground-based luminosity profile fits may place only upper limits on the scale lengths for some of the apparently smallest galaxies, hence the masses could be overestimated for a few slow rotators. In turn, the fit given above is likely to be conservative. Nevertheless, evidence for a mass-dependent luminosity evolution is found on the $4 \sigma$ confidence level (cf. Eq. 6.17).

The evolution corresponds to $\sim 2$ mag in restframe $B$ for the least massive objects and is negligible for high-mass spirals with $\sim 10^{12} M_{\odot}$. This implies that the enlargement of the TF offsets with increasing redshift represents a lower limit, since low-mass spirals — which show strong evolution in luminosity - exceed the constraint on apparent brightness from the target pre-selection. For the same reason, a decrease of the average galaxy masses with look-back time could not be tested. Note, however, that the virial mass for a given $V_{\max }$ does decrease with increasing redshift due to the linear dependence on the scale length (see chapter 5.4).

A parameter that has not been considered in the analysis of the FDF spirals is the metallicity. This was due to the low signal-to-noise ratio and the small number of usable lines in the spectra of most of the objects. The study of 64 late-type galaxies from the Deep Groth Strip Survey (Kobulnicky et al. 2003) points towards a combined evolution in luminosity and metallicity between $z \approx 1$ and $z=0$. Offsets from the local luminosity-metallicity relation found by this group are largest for galaxies in the lowluminosity regime $M_{B} \approx-19$. This would be in compliance with the TFR slope evolution observed in the FDF. However, since the absolute $B$-band magnitude is a poor mass tracer, a massdependence only is confirmed by this thesis. On the other hand, Vogt (2000) did not find a TFR slope change with a sample which was also selected from the Deep Groth Strip survey. 
Kobulnicky et al. (2003) compared their results to chemical enrichment models and found that the observations in the low-luminosity regime can be explained if at least two of the following assumptions are true:

1. low-mass galaxies have lower effective chemical yields than massive galaxies,

2. low-mass galaxies assemble on longer timescales than massive ones,

3. low-mass galaxies began the assembly process later than massive galaxies.

The negligible evolution observed in the highmass regime, however, has not been found in any of the simulations performed by the authors.

Similarly, a decreased TFR slope at intermediate redshift is in contradiction to theoretical predictions. Some models result in a slope that is independent of the cosmic age and only introduce a mild evolution towards higher luminosities with increasing $z$ (e.g., Steinmetz \& Navarro 1999). However, the reproduction of the local zero point value remains a challenge since the simulated Dark Matter halos are too dense at small radii (the "cuspy cores problem"). Simulations which use parameterisations of mass-dependent spectrochemical properties even yield an increase of the TFR slope with look-back time (Boissier \& Prantzos 2001, Ferreras \& Silk 2001).

If the observed shallower slope is interpreted in terms of younger ages, i.e., lower formation redshifts, for lower masses, the contradiction to the hierarchical scenario would be striking. This is because small Dark Matter halos should have formed earlier than large ones, and thus the stellar populations in galaxies of low mass should be older than those of high-mass systems.

It is well established and also observed for the FDF sample that the colors of spirals are redder (corresponding to earlier SED types) for higher masses, which is not reproduced by simulations within the hierarchical scenario, unless the spectrophotometric properties of local spirals are used as a calibration (Bell et al. 2003).
Further discrepancies between theoretical predictions and empirical studies arise from the small observed number of satellite galaxies in the extreme low-mass regime (the "missing satellites problem"). Keeping this in mind, the decreased TFR slope would "only" indicate additional shortcomings of the hierarchical model on small scales.

If the results of the TF analysis in the FDF point towards younger ages of the stellar populations in low-mass spirals than in high-mass spirals, how could this be explained? From the observational side, similar indications came from number counts in the $B$-band, which yielded an enhanced number density of objects that are small, blue and probably distant, known as the "faint blue galaxy excess" (an overview is given in Ellis 1997). These studies, however, were not reliable to discriminate between an overluminosity and an overdensity of the blue population. To explain the galaxy excess, it has been suggested that the UV background arising from Active Galactic Nuclei and starbursting galaxies could have suppressed the star formation in low-mass Dark Matter halos by a re-ionisation of the baryonic matter and, in turn, an increase of the Jeans mass. E.g., Babul \& Rees (1992) found that this mechanism is effective between redshifts $z \approx 6$ and $z \approx 1$ for halos with masses $M_{\text {vir }} \leq 10^{9} M_{\odot}$.

For the FDF spirals, however, this does not seem a likely explanation. The complete TF sample does comprise only galaxies with total masses $M_{\text {vir }} \geq 2.2 \cdot 10^{9} M_{\odot}$. To attribute the observed mass-dependence of the luminosity evolution to the UV background at $z>1$, the re-ionisation would have to be much more effective, and the upper halo mass limit would have to be $M_{\text {vir }} \approx 10^{11} M_{\odot}$. A modification of the simulated Dark Matter halos may be necessary to increase the upper mass limit by two orders of magnitude.

One major conclusion can be drawn from the results presented here: our understanding of 
the correlation between the depth of the gravitational potential well (the Dark Matter halo mass) and the properties of the stellar content (the baryons), which is observed in present-day and distant galaxies, has to be improved. Since the mechanisms that drive Star Formation on the smallest scales are also still a matter of debate (see Elmegreen 2003 for a review), and numerical simulations of galaxies by far have too small spatial resolutions to resolve individual stars, simplifying parameterisations are used for the modelling of stellar populations in current $N$-body codes. This most probably is one of the key issues for understanding the discrepancies between observations and theory on small spatial scales. A progress in this field may even lead to modifications of the hierarchical scenario itself.

It is hard to speculate on the improvements of our knowledge of galaxy formation and evolution in the years to come. More observational data are needed to strengthen the results of this thesis and other works. The steadily increasing computational power will allow to enhance the spatial resolution of future numerical simulations and to include a more realistic modelling of the stellar population properties.

In the near future, the project described in this thesis is going to be continued and the size of the sample will be enlarged by a factor of two with spectra of a further $\sim 100$ distant spirals observed with the VLT; see the outlook at the end of the following summary. 


\section{Chapter 7}

\section{Summary and Outlook}

Using imaging data and two-dimensional spectra taken with the ESO Very Large Telescope (VLT), a study of spiral galaxies at redshifts $0.1<z<1.0$ has been performed. This sample allowed to study the evolution of the field spiral population over the last $\sim 7$ Gyrs, corresponding to more than half the age of the universe. It was the aim of the project to quantify the galaxies' evolution in luminosity and size and thereby test theoretical predictions within the paradigm of modern cosmology, the Cold Dark Matter hierarchical scenario.

The objects were selected from the FORS Deep Field (FDF), a multi-band imaging study of a sky area near the southern galactic pole with optical brightness limits comparable to the Hubble Deep Fields and a field- - of-view $\sim 8$ times larger. The sample construction greatly benefitted from photometric redshifts and types which (at the time of the preparation of the observations) were available for $\sim 4000$ galaxies in the FDF. Selection effects that potentially bias a sample towards certain spectrophotometric types - as was the case in many previous observational studies at intermediate redshift — could thus be avoided. Targets for spectroscopy were selected solely on apparent $R$-band brightness with a faint limit of $R \leq 23$ mag. To minimize geometric distortions of the 2-D spectra, mainly spirals with disk inclinations of $i \geq 40^{\circ}$ were used. Moreover, an upper limit of $15^{\circ}$ on the misalignment angle between the apparent major axes and slit directions was adopted.
In total, spectra of 129 spiral galaxies were taken between 1999 and 2001 in Multi Object Spectroscopy mode with the FORS $1+2$ instruments mounted on the VLT. The seeing conditions ranged between 1.0 and 0.4 arcseconds. Spectroscopic redshifts and types could be derived for 113 spirals, while the spectra of 16 objects had too low signal-to-noise ratios.

The multi-band FDF imaging allowed a very accurate computation of the luminosities. Apparent brightnesses were derived in the passband which, depending on the redshift of a galaxy, best matched the rest-frame $B$-band. Type-dependent $k$-corrections were computed via synthetic photometry with spectral templates from chemically consistent evolutionary synthesis models. This resulted in very small uncertainties $\sigma_{k} \leq 0.1 \mathrm{mag}$ in the $k$-corrections over the complete redshift and spectral type ranges.

Disk inclinations, position angles and scale lengths were measured on a coadded $I$-band frame constructed with the FDF images of the best seeing $(0.5 \operatorname{arcsec})$. For the purpose of surface brightness profile fitting, an algorithm was developed which accounted for the Point Spread Function as well as a mild field distortion due to the focal reducer of the FORS instrument. To confirm that the ground-based imaging was appropriate for an accurate profile fitting, simulated VLT images of the Hubble Deep Field North have been created. The structural parameters derived on these frames were compared to 
results from the literature which were based on the original HST imaging, finding only mild deviations for the vast majority of the test sample. Spatially resolved Rotation Curves (RCs) of the FDF spirals were extracted from the [O II], [O III], $\mathrm{H} \beta$ or $\mathrm{H} \alpha$ emission lines. 36 of the RCs were rejected from the further analysis since they either had too low a radial extent, were asymmetric or did not show a regular rotation within the measured errors.

Particular effort was put into the development of a methodology for accurate derivations of the maximum rotation velocities $V_{\max }$ in the outer $\mathrm{RC}$ regions. In the approach adopted here, the velocity fields of the spirals were modelled, yielding synthetic rotation curves which were compared to the observed data. This procedure accounted for all geometric distortions, the seeing and the "beam smearing" effect due to the small apparent galaxy sizes.

$V_{\text {max }}$ could be derived for 77 FDF spirals. This Tully-Fisher sample comprised all spectrophotometric types from $\mathrm{Sa}$ to $\mathrm{Sdm} / \mathrm{Im}$ between $z=0.09$ and $z=0.97$ with a median redshift $\langle z\rangle=0.45$. The data were classified according to the spatial extent and symmetry of the rotation curves. Extensive consistency checks were performed by comparing different fitting methods between synthetic and observed RCs, $V_{\text {max }}$ values based on different emission lines and miscellaneous rotation velocity laws for the synthetic velocity field.

Morphological types were derived based on the asymmetry and concentration indices introduced by Abraham et al. (1996) and compared to the spectral types and RC properties. For the kinematically unpeculiar sample, it was found that the Hubble sequence of local galaxies was already established at redshift $z \approx 0.5$, in compliance with recent results from other studies.

In the further analysis, it has been shown that rotation curves with relatively small spatial extent can yield underestimated $V_{\max }$ values. This effect has not been taken into account in other distant spiral studies. To gain robust results, the constructions of the distant Tully-Fisher Relation (TFR) and the velocity-size relation were therefore restricted to curves which clearly reached into the "flat region" at large radii.

For a given maximum rotation velocity, the disks of the distant FDF spirals are, on the average, smaller by $20 \%$ than their local counterparts. Moreover, evidence was found for a dependence on redshift (look-back time) in the sense that disks at $z=1$ only have $60 \%$ the size of present-day spirals. These values are in very good agreement with the predictions of numerical simulations for a hierarchical universe. The slopes for the local and distant samples are consistent. However, the FDF velocity-size relation shows are large scatter which probably is induced by the limited spatial resolution of the groundbased imaging data.

The distant TFR, on the other hand, has a smaller slope than that of local samples at the $3 \sigma$ significance level. Since the $V_{\max }$ distribution of the FDF galaxies does not deviate from that of local spirals, the flatter tilt can be attributed to a mass-dependent luminosity evolution. Lowmass spirals are brighter by $\sim 2$ mag in restframe $B$ at $z \approx 0.5$ than locally, whereas the distant spirals with total masses $M_{\mathrm{vir}} \geq M_{\mathrm{vir}}^{*}$ have similar absolute magnitudes as their present-day counterparts.

To verify whether the analysis could be affected by systematic errors or peculiar properties of the distant spiral sample, a huge variety of tests were applied. It was found that neither of the following could have induced the observed change in the TFR slope:

- an incompleteness bias at the faint end of the observed luminosity distribution

- an evolution of the correlation between restframe colors and TF residuals

- enhanced Star Formation due to tidal galaxy-galaxy interactions, or 
- high Star Formation Rates for other reasons (e.g., accretion events)

- variations of the intrinsic RC shapes

- different assumptions on the intrinsic dust reddening

- the limited intrinsic spatial resolution

The large discrepancies between previous studies of the distant TFR could be easily explained on the basis of this result. Due to small number statistics, most of these studies had to assume a constant TFR slope. Moreover, many samples were biased by their selection criteria towards certain spectrophotometric sub-types. The FDF spirals show a correlation of these types with masses (which is well known for local galaxies): late-type spirals on the average are less massive than early-types. In combination with the decreased TFR slope, a correlation between type and luminosity evolution is implied. This most probably is the reason why samples which preferably contained late-type spirals yielded large deviations between the distant and present-day spiral luminosities, whereas studies biased towards earlier types only found a modest evolution.

The observed correlation between mass and restframe $B$-band brightening is at variance with the predictions of numerical simulations within the Cold Dark Matter hierarchical scenario. Some of these theoretical sudies pointed towards a constant TFR slope (e.g., Steinmetz \& Navarro 1999), while some groups even found a steepening with look-back time, i.e., an increase in luminosity that is larger for massive spirals than for low-mass spirals (e.g., Boissier \& Prantzos 2001, Ferreras \& Silk 2001).

It is unlikely that the re-ionisation era at redshifts $1<z<6$ and a corresponding suppressed Star Formation within low-mass Dark Matter halos in this regime could explain the results yielded by the FDF data. Upper limits on the halo mass range given in the literature would have to be larger by two orders of magnitude to be compatible with the observations.

Hence, this thesis may yield further evidence for shortcomings of the hierarchical scenario on small scales, of which the most prominent ones are the "cuspy cores problem" and the "missing satellites problem". The former term refers to the fact that the centers of simulated Dark Matter halos have steeper density profiles than is found in observations; the latter term depicts the large discrepancy between the predicted and observed number of Dark halo sub-clumbs (small satellite galaxies). Moreover, the observed colors of local high-mass spirals — which are redder than those of low-mass spirals - are not reproduced by numerical simulations unless these are calibrated to the spectrophotometric properties of present-day galaxies.

The results of this thesis thus strengthen the need for more realistic implementations of stellar population properties into the codes of $N$ body simulations. In particular, the fundamental physics causing the observed link between the Dark halo masses and the stellar populations still are not well understood.

It is hard to predict whether a more realistic modelling of the stellar content will lead to a consistency between observations and theory. Possibly, a modification of the Dark Matter hierarchical scenario would be needed. Further research in the years to come is going to be very important for our understanding of the formation and evolution of galaxies and, in turn, for the fundamental processes of structure growth from the very early universe to the present.

The project on distant spiral evolution described in this thesis will be continued in the next two years during a post-doctoral position of the author which is funded by the „Deutsches Zentrum für Luft- und Raumfahrt".

Observing time for VLT spectroscopy of an addi- 
tional $\sim 100$ spirals at $0.1<z<1.0$ has been granted by ESO. These objects were selected from the William Herschel Deep Field (Metcalfe et al. 2001), a deep photometric survey similar to the FDF performed with the William Herschel Telescope (Canary Islands). The target selection was based on estimated photometric redshifts and spectral types with the same constraints on inclinations and slit misalignment angles as for the FDF spirals. The new data will allow to enlarge the statistical significance of the results presented in this thesis.

Furthermore, imaging of the FDF and the William Herschel Deep Field with the new Advanced Camera for Surveys aboard the Hubble Space Telescope has been granted. Based on these high-resolution images, the morphological classification of the galaxies within the original Hubble scheme and the derivation of the bulgeto-total ratios will become feasible. The accuracy of the surface brightness profile fits will also benefit from the resolution of this imaging.

The combined spectroscopic data and spacebased images will make up the largest kinematic sample of intermediate-redshift spirals to date and allow deeper observational insights into the field of galaxy formation and evolution. 


\section{Appendix A}

\section{Spectrophotometric Data}

The tables presented in this part of the appendix contain the results of the spectrophotometric analysis which is described in chapter 4. Table A.1 comprises the 16 FORS Deep Field (FDF) spirals that did not yield a spectroscopic redshift, table A.2 contains all 36 spirals with derived redshifts which could not be used for the measurement of $V_{\max }$ due to the poor quality of their rotation curves. Table A.3 lists the data of the 77 spirals which yielded a value of $V_{\max }$. The respective columns in these three tables have the following meaning:

ID - FDF identification number as given in the photometric catalogue presented by Heidt et al. (2003).

$z$ - Spectroscopic redshift.

mod - SED parameter model as given in the August 2000 release of the FDF photometric redshifts catalogue, see chapter 2.3.2.

$z_{\text {phot }}, \sigma_{\text {zphot }}$ - Photometric redshift with corresponding error (only given for the objects without a spectroscopic redshift).

$E W$ - Equivalent width of the [O II] 3727 doublet (if visible), see chapter 4.2 .

$T$ - SED type in the de Vaucouleurs scheme, $T=1$ corresponds to Sa, $T=3$ to $\mathrm{Sb}, T=5$ to Sc, $T=8$ to $\mathrm{Sdm}$ and $T=10$ to Im. In the sub-sample of the 36 spirals without a value for $V_{\max }$, all very late-types are denoted Sdm.

$A, C$ - Asymmetry and concentration indices (introduced by Abraham et al. 1996). For blended objects, no value is given, see chap- ter 4.5.

$m_{B}, m_{g}, m_{R}, m_{I}$ - Total apparent magnitudes in the passbands of the respective FORS filters $B, g, R$ and $I$, as derived with the Source Extractor package (Bertin \& Arnouts 1996) on the coadded images of the FDF, see chapter 4.6.1. An empty entry indicates that the object is not visible in the field-of-view of the coadded image. $X$ - Passband used for the computation of the absolute magnitudes. Depending on a galaxy's redshift, this was the filter that best matched the rest-frame $B$-band, see chapter 4.6.4.

$A_{X}^{g}$ - Galactic absorption in the passband of filter $X$, see chapter 4.6.2.

$D M_{\Lambda}$ - Distance modulus in the concordance cosmology with $\Omega_{m}=0.3, \Omega_{\Lambda}=0.7$ and $H_{0}=70 \mathrm{~km} \mathrm{~s}^{-1} \mathrm{Mpc}^{-1}$.

$k_{B}-K$-correction for transformation from FORS filter $X$ to rest-frame Johnson $B_{J}$, see chapter 4.6 .4 and also tables A.5 to A.9 within this appendix.

$A_{B}^{i}$ - Intrinsic $B$-band absorption following Tully \& Fouqué (1985), see chapter 4.6.5.

$M_{B}$ - Absolute $B$-band magnitude, computed according to Eq. 4.21 in chapter 4.6.

$\sigma_{M B}$ - Error on absolute $B$-band magnitude, computed according to Eq. 4.32 in chapter 4.7. Note that these errors are given only for the 77 spirals with derived $V_{\max }$ values.

For the 16 galaxies without a spectroscopic redshift, only the columns ID, mod, $z_{\text {phot }}, \sigma_{\text {zphot }}$, $m_{B}, m_{g}, m_{R}$ and $m_{I}$ are given. 
Table A.1: Spectrophotometric data of the 16 spirals from the FORS Deep Field sample without a spectroscopic redshift. Some entries are empty since the corresponding objects were not included in the August 2000 release of the photometric redshifts catalogue or in the coadded images.

\begin{tabular}{rrrrrrrr}
\hline ID & mod & $z_{\text {phot }}$ & $\sigma_{\text {zphot }}$ & $\begin{array}{c}m_{B} \\
{[\mathrm{mag}]}\end{array}$ & $\begin{array}{c}m_{g} \\
{[\mathrm{mag}]}\end{array}$ & $\begin{array}{c}m_{R} \\
{[\mathrm{mag}]}\end{array}$ & $\begin{array}{c}m_{I} \\
{[\mathrm{mag}]}\end{array}$ \\
\hline & & & & & & & \\
\hline 510 & & & & 23.87 & 23.53 & 23.11 & 22.41 \\
593 & & & & 24.90 & & & 22.17 \\
1676 & 6 & 0.22 & 0.06 & 24.63 & 23.72 & 22.84 & 22.35 \\
2297 & 12 & 0.44 & 0.10 & 23.66 & 23.43 & 22.39 & 22.05 \\
3725 & 6 & 0.74 & 0.08 & 23.65 & 23.06 & 21.98 & 21.25 \\
4516 & 12 & 1.04 & 0.19 & 23.72 & 23.41 & 23.02 & 22.54 \\
4519 & 3 & 0.56 & 0.08 & 24.68 & 23.76 & 22.14 & 21.42 \\
5070 & 6 & 0.54 & 0.11 & 24.61 & 24.06 & 22.79 & 22.21 \\
5117 & 3 & 0.88 & 0.11 & 25.72 & 24.83 & 23.04 & 21.83 \\
5269 & 12 & 0.94 & 0.38 & 25.06 & 24.47 & 23.11 & 22.57 \\
5756 & 10 & 0.16 & 0.05 & 23.90 & 23.32 & 22.91 & 22.53 \\
5788 & 8 & 0.92 & 0.08 & 23.51 & 23.23 & 22.43 & 21.48 \\
6032 & 3 & 0.12 & 0.09 & 23.73 & 22.76 & 21.75 & 21.16 \\
6801 & 5 & 0.90 & 0.16 & 24.24 & 23.83 & 22.50 & 21.35 \\
8172 & 7 & 1.38 & 0.21 & 23.83 & 23.60 & 23.34 & 23.10 \\
8401 & & & & 24.81 & 23.95 & 22.31 & 21.62 \\
& & & & & & & \\
\hline
\end{tabular}

Table A.2: Spectrophotometric data of the 36 spirals from the FORS Deep Field sample with rotation curves that were not appropriate for a derivation of $V_{\max }$.

\begin{tabular}{|c|c|c|c|c|c|c|c|c|c|c|c|c|c|c|c|c|}
\hline ID & $z$ & mod & $\begin{array}{c}E W \\
{[\AA]}\end{array}$ & $T$ & $A$ & $C$ & $\begin{array}{c}m_{B} \\
{[\mathrm{mag}]}\end{array}$ & $\begin{array}{c}m_{g} \\
{[\mathrm{mag}]}\end{array}$ & $\begin{array}{c}m_{R} \\
{[\mathrm{mag}]}\end{array}$ & $\begin{array}{c}m_{I} \\
{[\mathrm{mag}]}\end{array}$ & $X$ & $\begin{array}{c}A_{X}^{g} \\
{[\mathrm{mag}]}\end{array}$ & $\begin{array}{l}D M_{\Lambda} \\
{[\mathrm{mag}]}\end{array}$ & $\begin{array}{c}k_{B} \\
{[\mathrm{mag}]}\end{array}$ & $\begin{array}{c}A_{B}^{i} \\
{[\mathrm{mag}]}\end{array}$ & $\begin{array}{c}M_{B} \\
{[\mathrm{mag}]}\end{array}$ \\
\hline 216 & 0.3947 & & & 8 & & & 23.55 & 22.96 & 22.03 & 21.66 & $g$ & 0.06 & 41.65 & +0.19 & 0.78 & -19.72 \\
\hline 543 & 0.2737 & & & 5 & 0.205 & 0.372 & 21.29 & 20.49 & 19.67 & 19.16 & $g$ & 0.06 & 40.73 & -0.15 & 0.32 & -20.47 \\
\hline 1072 & 0.7944 & 8 & 40.6 & 8 & 0.156 & 0.244 & 23.95 & 23.78 & 22.91 & 22.19 & $R$ & 0.04 & 43.49 & -0.36 & 0.40 & -20.66 \\
\hline 1422 & 0.8636 & 11 & 63.4 & 8 & 0.089 & 0.375 & 23.80 & 23.37 & 22.80 & 22.14 & $I$ & 0.04 & 43.71 & -1.12 & 0.42 & -20.90 \\
\hline 1870 & 0.0338 & 10 & & 8 & 0.105 & 0.415 & 20.47 & 20.08 & 19.63 & 19.33 & $B$ & 0.08 & 35.86 & +0.12 & 0.56 & -16.15 \\
\hline 1948 & 0.3908 & & 21.2 & 5 & 0.192 & 0.404 & 21.57 & & & 19.49 & $B$ & 0.08 & 41.63 & +1.02 & 0.40 & -21.56 \\
\hline 2228 & 0.4493 & 2 & 4.6 & 1 & 0.096 & 0.567 & 23.61 & 22.43 & 20.70 & 19.93 & $g$ & 0.06 & 41.99 & +0.51 & 0.34 & -20.47 \\
\hline 2398 & 0.3306 & 3 & 18.6 & 5 & 0.100 & 0.307 & 24.49 & 23.63 & 22.49 & 21.95 & $g$ & 0.06 & 41.20 & +0.02 & 0.34 & -18.00 \\
\hline 2847 & 0.2742 & 8 & & 8 & 0.124 & 0.273 & 23.37 & 22.52 & 21.80 & 21.38 & $g$ & 0.06 & 40.73 & -0.19 & 0.44 & -18.51 \\
\hline 3228 & 0.7840 & 2 & 2.3 & 1 & 0.174 & 0.446 & 24.90 & 24.47 & 21.84 & 20.22 & $R$ & 0.04 & 43.45 & -0.22 & 0.42 & -21.86 \\
\hline 3995 & 0.0665 & 10 & & 8 & 0.100 & 0.212 & 23.22 & 22.82 & 22.42 & 22.10 & $B$ & 0.08 & 37.38 & +0.22 & 0.32 & -14.78 \\
\hline 4271 & 0.4073 & 8 & & 5 & 0.163 & 0.212 & 23.69 & 23.12 & 22.07 & 21.64 & $g$ & 0.06 & 41.73 & +0.27 & 0.32 & -19.27 \\
\hline 4409 & 0.6117 & 7 & 50.5 & 8 & 0.197 & 0.303 & 23.47 & 23.01 & 21.94 & 21.44 & $R$ & 0.04 & 42.79 & -0.67 & 0.31 & -20.53 \\
\hline 4602 & 0.6530 & 4 & 3.8 & 1 & 0.119 & 0.248 & 24.42 & 23.80 & 22.39 & 21.49 & $R$ & 0.04 & 42.96 & -0.51 & 0.29 & -20.39 \\
\hline 4822 & 0.2244 & 0 & 42.5 & 8 & 0.115 & 0.333 & 23.22 & 22.45 & 21.78 & 21.34 & $B$ & 0.08 & 40.24 & +0.67 & 0.36 & -18.13 \\
\hline 4914 & 0.6843 & 3 & 11.8 & 3 & 0.190 & 0.340 & 25.32 & 24.47 & 22.93 & 22.06 & $R$ & 0.04 & 43.09 & -0.48 & 0.32 & -20.04 \\
\hline 4972 & 0.4064 & 6 & & 3 & 0.093 & 0.199 & 24.31 & 23.66 & 22.38 & 21.82 & $g$ & 0.06 & 41.73 & +0.34 & 0.45 & -18.93 \\
\hline 5055 & 0.6848 & 8 & 30.5 & 5 & 0.118 & 0.328 & 24.28 & 24.00 & 23.03 & 22.35 & $R$ & 0.04 & 43.09 & -0.52 & 0.31 & -19.89 \\
\hline 5096 & 0.3701 & 12 & & 8 & 0.125 & 0.318 & 23.67 & 23.18 & 22.35 & 22.04 & $g$ & 0.06 & 41.49 & +0.11 & 0.28 & -18.77 \\
\hline 5225 & 0.4524 & 10 & 38.4 & 8 & 0.134 & 0.323 & 22.49 & 22.11 & 21.13 & 20.74 & $g$ & 0.06 & 42.00 & +0.30 & 0.32 & -20.58 \\
\hline 5556 & 1.0715 & 9 & 9.6 & 5 & 0.263 & 0.241 & 24.72 & 24.33 & 23.85 & 23.15 & $I$ & 0.04 & 44.29 & -0.25 & 0.34 & -21.27 \\
\hline 5585 & 0.9405 & 7 & 8.1 & 5 & 0.302 & 0.196 & 23.07 & 22.59 & 21.83 & 20.97 & $I$ & 0.04 & 43.94 & -0.98 & 0.42 & -22.45 \\
\hline 5835 & 0.3367 & 11 & & 8 & 0.210 & 0.322 & 22.52 & 21.88 & 21.18 & 20.86 & $g$ & 0.06 & 41.25 & 0.00 & 0.40 & -19.82 \\
\hline 6486 & 0.3339 & 8 & & 8 & 0.399 & 0.225 & 23.74 & 23.07 & 22.17 & 21.74 & $g$ & 0.06 & 41.23 & -0.01 & 0.49 & -18.70 \\
\hline 6597 & 0.6860 & 6 & 38.3 & 5 & 0.178 & 0.269 & 22.99 & 22.64 & 21.72 & 21.04 & $R$ & 0.04 & 43.09 & -0.52 & 0.31 & -21.20 \\
\hline 6649 & 0.2810 & 5 & & 5 & 0.116 & 0.245 & 23.93 & 23.19 & 22.41 & 21.82 & $g$ & 0.06 & 40.79 & -0.13 & 0.44 & -17.97 \\
\hline 7105 & 0.3897 & 8 & 26.6 & 8 & 0.142 & 0.288 & 24.05 & 23.57 & 22.76 & 22.41 & $g$ & 0.06 & 41.62 & +0.17 & 0.78 & -19.07 \\
\hline 7167 & 0.4106 & 10 & & 8 & 0.096 & 0.200 & 24.37 & 23.76 & 22.87 & 22.59 & $g$ & 0.06 & 41.75 & +0.23 & 0.40 & -18.68 \\
\hline 7024 & 0.8715 & 9 & 22.8 & 5 & 0.115 & 0.270 & 23.90 & 23.60 & 22.85 & 22.07 & $I$ & 0.04 & 43.73 & -1.10 & 0.28 & -20.87 \\
\hline 7186 & 0.4481 & & 28.4 & 5 & 0.152 & 0.270 & 23.06 & 22.63 & 21.55 & 21.09 & $g$ & 0.06 & 41.98 & +0.34 & 0.37 & -20.12 \\
\hline 7457 & 0.3325 & 12 & & 8 & 0.197 & 0.187 & 23.56 & 23.05 & 22.34 & 22.39 & $g$ & 0.06 & 41.22 & -0.02 & 0.36 & -18.57 \\
\hline 7871 & 0.7607 & & & 5 & 0.139 & 0.272 & 25.36 & 24.41 & 22.75 & 21.66 & $R$ & 0.04 & 43.37 & -0.38 & 0.36 & -20.64 \\
\hline 7959 & 0.3377 & 3 & 27.9 & 5 & 0.110 & 0.372 & 23.40 & 22.24 & 20.97 & 20.37 & $g$ & 0.06 & 41.25 & +0.05 & 0.37 & -19.49 \\
\hline 8236 & 0.9384 & & & 8 & 0.096 & 0.305 & 23.44 & 23.26 & 22.87 & 22.31 & $I$ & 0.04 & 43.93 & -1.02 & 0.36 & -20.99 \\
\hline 8305 & 0.8450 & 7 & 18.3 & 5 & 0.291 & 0.211 & 23.75 & 22.41 & 21.62 & 20.84 & $R$ & 0.04 & 43.65 & -0.24 & 0.29 & -22.12 \\
\hline 8336 & 0.2736 & 6 & & 5 & 0.070 & 0.357 & 23.07 & 22.26 & 21.41 & 20.90 & $g$ & 0.06 & 40.73 & -0.15 & 0.28 & -18.66 \\
\hline
\end{tabular}


Table A.3: Spectrophotometric data of the 77 spirals from the FORS Deep Field Tully-Fisher sample with rotation curves that could be used for the derivation of $V_{\max }$.

\begin{tabular}{|c|c|c|c|c|c|c|c|c|c|c|c|c|c|c|c|c|c|}
\hline ID & $z$ & $\bmod$ & $\begin{array}{c}E W \\
{[\AA]}\end{array}$ & $T$ & $A$ & $C$ & $\begin{array}{c}m_{B} \\
{[\mathrm{mag}]}\end{array}$ & $\begin{array}{c}m_{g} \\
{[\mathrm{mag}]}\end{array}$ & $\begin{array}{c}m_{R} \\
{[\mathrm{mag}]}\end{array}$ & $\begin{array}{c}m_{I} \\
{[\mathrm{mag}]}\end{array}$ & $X$ & $\begin{array}{c}A_{X}^{g} \\
{[\mathrm{mag}]}\end{array}$ & $\begin{array}{l}D M_{\Lambda} \\
{[\mathrm{mag}]}\end{array}$ & $\begin{array}{c}k_{B} \\
{[\mathrm{mag}]}\end{array}$ & $\begin{array}{c}A_{B}^{i} \\
{[\mathrm{mag}]}\end{array}$ & $\begin{array}{c}M_{B} \\
{[\mathrm{mag}]}\end{array}$ & $\begin{array}{c}\sigma_{M B} \\
{[\mathrm{mag}]}\end{array}$ \\
\hline 400 & 0.4483 & & 60.5 & 10 & 0.379 & 0.231 & 23.16 & 22.56 & & 21.37 & $g$ & 0.06 & 41.98 & +0.18 & 0.49 & -20.15 & 0.13 \\
\hline 745 & 0.6986 & 7 & 16.5 & 5 & & & 22.56 & 22.10 & 21.06 & 20.35 & $\stackrel{P}{R}$ & 0.04 & 43.14 & -0.49 & 0.35 & -21.98 & 0.08 \\
\hline 870 & 0.2775 & 8 & 33.4 & 5 & 0.187 & 0.354 & 21.50 & 20.70 & 20.06 & 19.63 & $g$ & 0.06 & 40.76 & -0.14 & 0.42 & -20.40 & 0.08 \\
\hline 1082 & 0.4482 & 8 & 38.1 & 8 & 0.272 & 0.290 & 22.57 & 22.07 & 21.07 & 20.59 & $g$ & 0.06 & 41.98 & +0.29 & 0.39 & -20.66 & 0.08 \\
\hline 1224 & 0.3989 & 12 & 96.9 & 10 & 0.088 & 0.238 & 23.63 & 23.19 & 22.43 & 22.30 & $g$ & 0.06 & 41.68 & +0.09 & 0.39 & -19.03 & 0.13 \\
\hline 1327 & 0.3141 & 7 & & 3 & 0.097 & 0.358 & 21.24 & 20.43 & 19.56 & 19.08 & $g$ & 0.06 & 41.07 & +0.04 & 0.31 & -21.05 & 0.07 \\
\hline 1449 & 0.1140 & 7 & & 5 & 0.088 & 0.612 & 20.52 & 19.73 & 19.06 & 18.55 & $\stackrel{S}{B}$ & 0.08 & 38.62 & +0.40 & 0.33 & -18.90 & 0.08 \\
\hline 1476 & 0.4360 & 3 & 9.0 & 3 & 0.135 & 0.336 & 23.49 & 22.65 & 21.18 & 20.46 & $g$ & 0.06 & 41.91 & +0.40 & 0.85 & -20.58 & 0.09 \\
\hline 1569 & 0.4625 & 9 & 41.6 & 8 & 0.163 & 0.153 & 24.52 & 24.09 & 23.06 & 22.63 & $g$ & 0.06 & 42.06 & +0.32 & 0.33 & -18.69 & 0.13 \\
\hline 1625 & 0.2304 & 4 & 18.8 & 5 & 0.141 & 0.388 & 23.37 & 22.46 & 21.55 & 20.92 & $\stackrel{\leftrightarrow}{B}$ & 0.08 & 40.30 & $\begin{array}{r}0.74 \\
\end{array}$ & 0.46 & -18.20 & 0.09 \\
\hline 1655 & 0.3377 & 8 & & 5 & 0.111 & 0.318 & 22.52 & 21.81 & 21.02 & 20.61 & $g$ & 0.06 & 41.25 & +0.05 & 0.41 & -19.96 & 0.08 \\
\hline 1699 & 0.2299 & 11 & & 8 & 0.124 & 0.364 & 22.67 & 22.07 & 21.53 & 21.15 & $B$ & 0.08 & 40.30 & +0.68 & 0.38 & -18.77 & 0.13 \\
\hline 1834 & 0.3475 & 4 & 22.5 & 5 & 0.135 & 0.227 & 24.69 & 23.99 & 22.86 & 22.08 & $g$ & 0.06 & 41.33 & +0.08 & 0.39 & -17.88 & 0.13 \\
\hline 1928 & 0.7179 & 12 & 63.7 & 10 & 0.090 & 0.222 & 23.84 & 23.59 & 22.89 & 22.33 & $R$ & 0.04 & 43.22 & -0.46 & 0.49 & -20.40 & 0.13 \\
\hline 2007 & 0.7175 & 10 & 51.3 & 10 & 0.126 & 0.200 & 23.87 & 23.50 & 22.68 & 22.09 & $R$ & 0.04 & 43.21 & -0.46 & 0.52 & -20.63 & 0.11 \\
\hline 2067 & 0.7942 & 7 & 23.9 & 5 & 0.158 & 0.260 & 23.35 & 22.79 & 21.87 & 21.07 & $R$ & 0.04 & 43.48 & -0.32 & 0.32 & -21.65 & 0.08 \\
\hline 2174 & 0.6798 & 4 & 15.7 & 3 & 0.188 & 0.343 & 23.78 & 23.32 & 22.08 & 21.23 & $R$ & 0.04 & 43.07 & -0.49 & 0.46 & -21.00 & 0.09 \\
\hline 2246 & 0.6514 & 7 & 14.8 & 5 & 0.138 & 0.315 & 22.98 & 22.47 & 21.52 & 20.78 & $R$ & 0.04 & 42.96 & -0.58 & 0.37 & -21.27 & 0.09 \\
\hline 2328 & 0.3956 & 0 & 41.5 & 8 & 0.127 & 0.314 & 23.06 & 22.37 & 21.46 & 21.06 & $g$ & 0.06 & 41.66 & +0.19 & 0.61 & -20.16 & 0.11 \\
\hline 2341 & 0.7611 & 4 & 27.7 & 5 & 0.165 & 0.321 & 23.90 & 23.07 & 22.09 & 21.22 & $R$ & 0.04 & 43.37 & -0.38 & 0.31 & -21.25 & 0.09 \\
\hline 2353 & 0.7773 & 4 & 23.4 & 3 & 0.137 & 0.415 & 23.68 & 23.13 & 22.05 & 21.17 & $R$ & 0.04 & 43.43 & -0.29 & 0.35 & -21.48 & 0.09 \\
\hline 2397 & 0.4519 & 9 & 27.7 & 5 & 0.219 & 0.278 & 22.35 & 21.91 & 20.84 & 20.42 & $g$ & 0.06 & 42.00 & +0.35 & 0.34 & -20.84 & 0.08 \\
\hline 2484 & 0.6535 & 6 & 7.8 & 5 & 0.130 & 0.261 & 23.45 & 22.94 & 21.78 & 20.98 & $R$ & 0.04 & 42.97 & -0.58 & 0.41 & -21.07 & 0.09 \\
\hline 2533 & 0.3150 & 7 & 27.1 & 5 & 0.178 & 0.372 & 21.11 & 20.35 & 19.61 & 19.15 & $g$ & 0.06 & 41.08 & -0.03 & 0.56 & -21.32 & 0.09 \\
\hline 2572 & 0.4491 & 7 & 28.9 & 5 & 0.152 & 0.288 & 23.44 & 22.93 & 21.85 & 21.40 & $g$ & 0.06 & 41.98 & +0.34 & 0.45 & -19.90 & 0.10 \\
\hline 2574 & 0.6802 & 0 & 40.7 & 5 & 0.170 & 0.243 & 24.40 & 24.00 & 22.98 & 22.33 & $R$ & 0.04 & 43.07 & -0.53 & 0.61 & -20.22 & 0.13 \\
\hline 2783 & 0.3143 & 8 & & 5 & 0.122 & 0.405 & 22.28 & 21.50 & 20.77 & 20.36 & $g$ & 0.06 & 41.07 & -0.03 & 0.35 & -19.95 & 0.08 \\
\hline 2800 & 0.6290 & 10 & 39.3 & 8 & 0.323 & 0.206 & 23.55 & 23.25 & 22.23 & 21.70 & $R$ & 0.04 & 42.87 & -0.64 & 0.38 & -20.42 & 0.09 \\
\hline 2822 & 0.5871 & 8 & 33.2 & 8 & 0.161 & 0.235 & 23.17 & 22.77 & 21.80 & 21.29 & $R$ & 0.04 & 42.68 & -0.71 & 0.41 & -20.62 & 0.09 \\
\hline 2946 & 0.7437 & 7 & 52.5 & 10 & 0.112 & 0.238 & 23.92 & 23.61 & 22.99 & 22.48 & $R$ & 0.04 & 43.31 & -0.42 & 0.45 & -20.38 & 0.13 \\
\hline 2958 & 0.3139 & 8 & 39.4 & 5 & 0.253 & 0.296 & 22.16 & 21.41 & 20.71 & 20.35 & $g$ & 0.06 & 41.07 & -0.03 & 0.44 & -20.13 & 0.08 \\
\hline 3071 & 0.0939 & 6 & & 5 & 0.063 & 0.705 & 19.53 & 18.82 & 18.11 & 17.60 & $\stackrel{S}{B}$ & 0.08 & 38.17 & +0.34 & 0.34 & -19.39 & 0.08 \\
\hline 3108 & 0.4741 & 11 & 15.8 & 5 & 0.099 & 0.220 & 23.79 & 23.26 & 22.10 & 21.57 & $g$ & 0.06 & 42.12 & +0.39 & 0.50 & -19.81 & 0.11 \\
\hline 3131 & 0.7723 & 8 & 42.5 & 5 & 0.222 & 0.259 & 23.80 & 23.27 & 22.47 & 21.78 & $R$ & 0.04 & 43.41 & -0.36 & 0.43 & -21.05 & 0.12 \\
\hline 3578 & 0.7718 & 0 & 15.1 & 5 & 0.180 & 0.239 & & & 22.68 & 21.63 & $R$ & 0.04 & 43.41 & -0.36 & 0.50 & -20.91 & 0.13 \\
\hline 3704 & 0.4082 & 4 & & 3 & 0.105 & 0.295 & 24.51 & 23.85 & 22.62 & 22.03 & $g$ & 0.06 & 41.74 & +0.35 & 0.96 & -19.27 & 0.16 \\
\hline 3730 & 0.9593 & 6 & 34.4 & 5 & 0.170 & 0.249 & 24.67 & & 23.68 & 22.97 & $I$ & 0.04 & 43.99 & -0.95 & 0.61 & -20.72 & 0.15 \\
\hline 3921 & 0.2251 & 0 & 7.5 & 3 & 0.227 & 0.402 & 19.90 & 18.71 & 17.93 & 17.39 & $B$ & 0.08 & 40.25 & +0.82 & 0.48 & -21.73 & 0.11 \\
\hline 4113 & 0.3951 & 5 & 10.2 & 1 & 0.100 & 0.306 & 23.74 & 22.97 & 21.77 & 21.22 & $g$ & 0.06 & 41.65 & +0.39 & 0.63 & -19.77 & 0.12 \\
\hline 4371 & 0.4605 & 5 & 8.5 & 3 & 0.125 & 0.329 & 23.67 & 23.06 & 21.75 & 21.16 & $g$ & 0.06 & 42.05 & +0.45 & 0.30 & -19.80 & 0.09 \\
\hline 4376 & 0.3961 & 9 & 48.2 & 8 & 0.163 & 0.252 & 24.29 & 23.65 & 22.72 & 22.36 & $g$ & 0.06 & 41.66 & +0.20 & 0.67 & -18.94 & 0.11 \\
\hline 4402 & 0.1138 & 4 & & 3 & 0.090 & 0.464 & 20.24 & 19.36 & 18.57 & 18.03 & $B$ & 0.08 & 38.62 & +0.47 & 0.78 & -19.71 & 0.09 \\
\hline 4465 & 0.6117 & 4 & 25.4 & 5 & 0.165 & 0.300 & 23.57 & 22.71 & 21.56 & 20.92 & $R$ & 0.04 & 42.79 & -0.65 & 0.50 & -21.12 & 0.10 \\
\hline 4498 & 0.7827 & 8 & 33.4 & 8 & 0.156 & 0.255 & 23.84 & 23.45 & 22.60 & 21.82 & $R$ & 0.04 & 43.45 & -0.38 & 0.34 & -20.85 & 0.11 \\
\hline 4657 & 0.2248 & 4 & & 5 & 0.118 & 0.461 & 21.79 & 20.78 & 19.89 & 19.31 & $B$ & 0.08 & 40.24 & +0.73 & 0.33 & -19.58 & 0.08 \\
\hline 4730 & 0.7820 & 3 & 3.7 & 1 & 0.182 & 0.478 & 23.19 & 22.40 & 20.94 & 19.79 & $R$ & 0.04 & 43.44 & -0.23 & 0.31 & -22.63 & 0.08 \\
\hline 4806 & 0.2214 & 5 & & 5 & 0.135 & 0.391 & 22.07 & 21.10 & 20.30 & 19.78 & $B$ & 0.08 & 40.21 & +0.72 & 0.56 & -19.50 & 0.08 \\
\hline 4922 & 0.9731 & 10 & 35.3 & 10 & 0.227 & 0.185 & 23.33 & 23.04 & 22.48 & 21.77 & $I$ & 0.04 & 44.03 & -1.06 & 0.54 & -21.78 & 0.11 \\
\hline 5022 & 0.3385 & 12 & & 10 & 0.149 & 0.188 & 23.55 & 22.87 & 22.21 & 21.96 & $g$ & 0.06 & 41.26 & -0.11 & 0.72 & -19.06 & 0.14 \\
\hline 5140 & 0.2738 & 7 & & 5 & 0.126 & 0.272 & 23.68 & 22.87 & 22.23 & 21.79 & $g$ & 0.06 & 40.73 & -0.15 & 0.40 & -18.16 & 0.10 \\
\hline 5286 & 0.3337 & 8 & & 8 & 0.098 & 0.230 & 24.46 & 23.81 & 23.01 & 22.63 & $g$ & 0.06 & 41.22 & -0.01 & 0.56 & -18.02 & 0.18 \\
\hline 5317 & 0.9745 & 8 & 26.8 & 5 & 0.219 & 0.221 & 23.42 & 23.00 & 22.37 & 21.39 & $I$ & 0.04 & 44.03 & -0.92 & 0.42 & -22.18 & 0.09 \\
\hline 5335 & 0.7726 & 7 & 30.4 & 5 & 0.111 & 0.269 & 23.87 & 23.42 & 22.53 & 21.77 & $R$ & 0.04 & 43.41 & -0.36 & 0.31 & -20.86 & 0.10 \\
\hline 5361 & 0.3339 & 6 & & 5 & & & 23.16 & 22.38 & 21.41 & 20.90 & $g$ & 0.06 & 41.23 & +0.03 & 0.37 & -19.32 & 0.09 \\
\hline 5515 & 0.8934 & 4 & 6.7 & 3 & 0.131 & 0.286 & 23.95 & 23.48 & 22.39 & 21.33 & $I$ & 0.04 & 43.80 & -1.03 & 0.39 & -21.86 & 0.10 \\
\hline 5565 & 0.2285 & 10 & & 8 & 0.155 & 0.308 & 23.55 & 22.68 & 22.18 & 21.82 & $B$ & 0.08 & 40.28 & +0.68 & 0.53 & -18.01 & 0.11 \\
\hline 6125 & 0.4495 & 5 & 20.6 & 5 & 0.072 & 0.466 & 23.81 & 22.71 & 21.14 & 20.47 & $g$ & 0.06 & 41.99 & +0.34 & 0.46 & -20.14 & 0.08 \\
\hline 6253 & 0.3453 & 8 & & 5 & 0.134 & 0.097 & 24.50 & 23.68 & 23.08 & 22.56 & $g$ & 0.06 & 41.31 & +0.07 & 0.81 & -18.58 & 0.19 \\
\hline 6406 & 0.8451 & 6 & 11.1 & 3 & 0.134 & 0.228 & 23.02 & 22.54 & 21.65 & 20.79 & R & 0.04 & 43.65 & -0.16 & 0.37 & -22.25 & 0.08 \\
\hline 6452 & 0.3359 & 9 & & 5 & 0.129 & 0.280 & 23.68 & 23.08 & 22.32 & 21.88 & $g$ & 0.06 & 41.24 & +0.04 & 0.37 & -18.63 & 0.11 \\
\hline 6568 & 0.4597 & 6 & 17.3 & 5 & 0.107 & 0.232 & 23.37 & 22.78 & 21.56 & 20.98 & $g$ & 0.06 & 42.04 & +0.36 & 0.42 & -20.11 & 0.09 \\
\hline 6585 & 0.3357 & 3 & & 5 & 0.176 & 0.438 & 21.10 & 20.19 & 19.17 & 18.62 & $g$ & 0.06 & 41.24 & +0.04 & 0.67 & -21.83 & 0.08 \\
\hline 6657 & 0.3343 & 5 & & 3 & 0.116 & 0.424 & 21.71 & 20.92 & 19.89 & 19.32 & $g$ & 0.06 & 41.23 & +0.11 & 0.30 & -20.78 & 0.07 \\
\hline 6743 & 0.7320 & 10 & 47.2 & 8 & 0.128 & 0.245 & 23.72 & 23.37 & 22.73 & 22.08 & $R$ & 0.04 & 43.27 & -0.47 & 0.34 & -20.46 & 0.10 \\
\hline 6921 & 0.4538 & 8 & & 5 & 0.103 & 0.253 & 23.98 & 23.40 & 22.34 & 21.88 & $g$ & 0.06 & 42.01 & +0.35 & 0.38 & -19.40 & 0.12 \\
\hline 7298 & 0.3902 & 7 & & 5 & 0.144 & 0.289 & 23.46 & 21.41 & 20.36 & 19.88 & $g$ & 0.06 & 41.62 & +0.22 & 0.32 & -20.81 & 0.09 \\
\hline 7429 & 0.3370 & 8 & 28.8 & 5 & 0.087 & 0.352 & 22.34 & 21.61 & 20.82 & 20.42 & $g$ & 0.06 & 41.25 & +0.04 & 0.44 & -20.19 & 0.09 \\
\hline 7526 & 0.3589 & 7 & & 5 & 0.255 & 0.319 & 21.54 & 20.79 & 19.89 & 19.46 & $g$ & 0.06 & 41.41 & +0.12 & 0.48 & -21.28 & 0.07 \\
\hline 7597 & 0.4096 & 7 & & 5 & 0.100 & 0.322 & 24.81 & 24.17 & 23.12 & 22.66 & $g$ & 0.06 & 41.75 & +0.27 & 0.61 & -18.52 & 0.21 \\
\hline 7725 & 0.5504 & 9 & & 8 & 0.208 & 0.294 & 23.51 & 23.17 & 22.18 & 21.71 & $R$ & 0.04 & 42.51 & -0.77 & 0.52 & -20.12 & 0.12 \\
\hline 7733 & 0.4471 & & & 5 & 0.181 & 0.200 & 23.27 & 22.72 & 21.57 & 21.34 & $g$ & 0.06 & 41.97 & +0.34 & 0.41 & -20.06 & 0.09 \\
\hline 7856 & 0.5146 & 3 & 13.4 & 5 & 0.082 & 0.324 & 23.21 & 22.56 & 21.24 & 20.59 & $g$ & 0.06 & 42.32 & +0.45 & 0.43 & -20.69 & 0.09 \\
\hline 7866 & 0.2240 & 3 & & 3 & & & 23.11 & 22.28 & 21.29 & 20.66 & $B$ & 0.08 & 40.23 & +0.82 & 0.42 & -18.44 & 0.10 \\
\hline 8034 & 0.7317 & 6 & 33.1 & 5 & 0.117 & 0.398 & 23.63 & 23.21 & 22.34 & 21.69 & $R$ & 0.04 & 43.27 & -0.43 & 0.35 & -20.89 & 0.11 \\
\hline 8190 & 0.3947 & 6 & & 5 & 0.118 & 0.307 & 22.39 & 21.77 & 20.81 & 20.39 & $g$ & 0.06 & 41.65 & +0.24 & 0.37 & -20.55 & 0.08 \\
\hline 8360 & 0.7034 & 0 & 70.5 & 10 & 0.195 & 0.171 & 23.62 & 23.36 & 22.58 & 21.97 & $R$ & 0.04 & 43.16 & -0.48 & 0.53 & -20.67 & 0.16 \\
\hline 8526 & 0.6095 & 0 & & 5 & 0.173 & 0.334 & 23.11 & & 21.90 & 21.46 & $R$ & 0.04 & 42.78 & -0.66 & 0.67 & -20.94 & 0.14 \\
\hline
\end{tabular}


The structural parameters as derived with the program diskmodel (see chapter 4.3) are given for the 77 spirals with derived $V_{\max }$ values in table A.4. The respective columns have the following meaning:

ID - FDF identification number as given in the photometric catalogue presented by Heidt et al. (2003).

$m_{I}$ - Total apparent magnitude in the $I$-band, measured with the Source Extractor.

$\nu$ - Number of pixels of the thumbnail image used for the surface brightness profile fitting. $\chi_{\min }^{2}-$ Minimum $\chi^{2}$ of the best-fitting profile. $\chi_{\text {red }}^{2}$ - Reduced minimum $\chi^{2}$ of the best-fitting profile.

$i$ - Best-fitting disk inclination angle.

$\sigma_{i}$ - Error of the best-fitting disk inclination angle.

$r_{\mathrm{d}}$ [arcsec] - Best-fitting apparent disk scale length.

$\sigma_{\text {rd }}-$ Error of the best-fitting apparent disk scale length.

$r_{\mathrm{d}}[\mathrm{kpc}]$ - Best-fitting absolute disk scale length.

$\delta$ - Slit misalignment angle derived from the best-fitting disk position angle via Eq. 4.8 in chapter 4.3.

$\sigma_{\delta}$ - Error of the misalignment angle.

qual - Quality of the corresponding rotation curve, "H" denotes the high quality data, "L" represents low quality curves, see chapter 5.2.2 for the definitions.

Tables A.5 to A.9 give the $k$-corrections for types $\mathrm{Sa}, \mathrm{Sb}, \mathrm{Sc}, \mathrm{Sdm}$ and $\mathrm{Im}$, computed via synthetic photometry as described in chapter 4.6.4. The columns of these tables have the following contents:

$z$ - Redshift with an incremental stepsize of $\Delta z=0.1$.

$B \rightarrow B_{J}-K$-correction for transformation from FORS $B$ in observer's frame to Johnson $B_{J}$ in rest-frame.

$g \rightarrow B_{J}-K$-correction for transformation from FORS $g$ in observer's frame to Johnson $B_{J}$ in rest-frame.

$R \rightarrow B_{J}-K$-correction for transformation from FORS $R$ in observer's frame to Johnson $B_{J}$ in rest-frame.

$I \rightarrow B_{J}-K$-correction for transformation from FORS $I$ in observer's frame to Johnson $B_{J}$ in rest-frame.

For comparison, the $k$-corrections within the standard Johnson-Cousins filter system are also given:

$B_{J} \rightarrow B_{J}-K$-correction for transformation from Johnson $B_{J}$ in observer's frame to Johnson $B_{J}$ in rest-frame.

$V_{J} \rightarrow V_{J}-K$-correction for transformation from Johnson $V_{J}$ in observer's frame to Johnson $V_{J}$ in rest-frame.

$R_{C} \rightarrow R_{C}-K$-correction for transformation from Cousins $R_{C}$ in observer's frame to Cousins $R_{C}$ in rest-frame.

$I_{C} \rightarrow I_{C}-K$-correction for transformation from Cousins $I_{C}$ in observer's frame to Cousins $R_{C}$ in rest-frame. 
Table A.4: The structural parameters as derived with diskmodel for the 77 spirals from the FORS Deep Field Tully-Fisher sample.

\begin{tabular}{|c|c|c|c|c|c|c|c|c|c|c|c|c|}
\hline ID & $\begin{array}{c}m_{I} \\
{[\mathrm{mag}]}\end{array}$ & $\nu$ & $\chi_{\min }^{2}$ & $\chi_{\mathrm{red}}^{2}$ & $\begin{array}{c}i \\
{[\mathrm{deg}]}\end{array}$ & $\begin{array}{c}\sigma_{i} \\
{[\mathrm{deg}]}\end{array}$ & $\begin{array}{c}r_{\mathrm{d}} \\
{[\operatorname{arcsec}]}\end{array}$ & $\begin{array}{c}\sigma_{\mathrm{rd}} \\
{[\operatorname{arcsec}]}\end{array}$ & $\begin{array}{c}r_{\mathrm{d}} \\
{[\mathrm{kpc}]}\end{array}$ & $\begin{array}{c}\delta \\
{[\mathrm{deg}]}\end{array}$ & $\begin{array}{c}\sigma_{\delta} \\
{[\mathrm{deg}]}\end{array}$ & qual \\
\hline 400 & 21.37 & 961 & 1041.0 & 1.083 & 60 & 3 & 0.70 & 0.05 & 4.03 & +23 & 11 & $\mathrm{~L}$ \\
\hline 745 & 20.35 & 441 & 485.2 & 1.100 & 42 & 5 & 0.49 & 0.03 & 3.47 & +61 & 12 & L \\
\hline 870 & 19.63 & 441 & 296.1 & 0.671 & 53 & 3 & 0.53 & 0.03 & 2.24 & -8 & 6 & $\mathrm{H}$ \\
\hline 1082 & 20.59 & 441 & 372.2 & 0.844 & 49 & 5 & 0.50 & 0.05 & 2.88 & -10 & 11 & $\mathrm{H}$ \\
\hline 1224 & 22.30 & 441 & 392.4 & 0.890 & 49 & 13 & 0.34 & 0.05 & 1.82 & +5 & 36 & $\mathrm{~L}$ \\
\hline 1327 & 19.08 & 961 & 760.2 & 0.791 & 31 & 5 & 0.62 & 0.01 & 2.85 & -1 & 13 & $\mathrm{~L}$ \\
\hline 1449 & 18.55 & 961 & 564.4 & 0.587 & 36 & 4 & 0.66 & 0.06 & 1.37 & -30 & 13 & $\mathrm{~L}$ \\
\hline 1476 & 20.46 & 441 & 288.1 & 0.653 & 77 & 1 & 0.80 & 0.09 & 4.53 & -10 & 5 & L \\
\hline 1569 & 22.63 & 441 & 392.1 & 0.889 & 37 & 20 & 0.34 & 0.07 & 1.99 & +3 & 21 & $\mathrm{~L}$ \\
\hline 1625 & 20.92 & 441 & 369.1 & 0.837 & 57 & 4 & 0.38 & 0.01 & 1.40 & +10 & 14 & $\mathrm{~L}$ \\
\hline 1655 & 20.61 & 441 & 311.1 & 0.705 & 51 & 2 & 0.49 & 0.04 & 2.37 & 0 & 9 & $\mathrm{~L}$ \\
\hline 1699 & 21.15 & 441 & 363.2 & 0.824 & 48 & 14 & 0.21 & 0.05 & 0.77 & -11 & 35 & $\mathrm{~L}$ \\
\hline 1834 & 22.08 & 121 & 58.1 & 0.480 & 49 & 13 & 0.25 & 0.03 & 1.23 & +7 & 29 & L \\
\hline 1928 & 22.33 & 121 & 34.5 & 0.285 & 60 & 8 & 0.29 & 0.05 & 2.09 & +12 & 26 & $\mathrm{~L}$ \\
\hline 2007 & 22.09 & 121 & 40.7 & 0.337 & 62 & 5 & 0.40 & 0.06 & 2.89 & -5 & 15 & $\mathrm{~L}$ \\
\hline 2067 & 21.07 & 441 & 375.8 & 0.852 & 35 & 9 & 0.40 & 0.04 & 3.00 & -19 & 23 & $\mathrm{H}$ \\
\hline 2174 & 21.23 & 441 & 401.6 & 0.911 & 57 & 5 & 0.43 & 0.03 & 3.04 & +5 & 10 & $\mathrm{H}$ \\
\hline 2246 & 20.78 & 441 & 599.9 & 1.360 & 45 & 7 & 0.60 & 0.04 & 4.16 & 0 & 12 & $\mathrm{H}$ \\
\hline 2328 & 21.06 & 441 & 404.7 & 0.918 & 68 & 4 & 0.50 & 0.06 & 2.67 & -10 & 6 & $\mathrm{~L}$ \\
\hline 2341 & 21.22 & 441 & 408.3 & 0.926 & 32 & 13 & 0.35 & 0.03 & 2.58 & -5 & 36 & $\mathrm{H}$ \\
\hline 2353 & 21.17 & 441 & 407.1 & 0.923 & 41 & 8 & 0.50 & 0.07 & 3.72 & -9 & 21 & $\mathrm{H}$ \\
\hline 2397 & 20.42 & 961 & 1217.4 & 1.267 & 40 & 5 & 0.80 & 0.05 & 4.62 & -25 & 13 & $\mathrm{H}$ \\
\hline 2484 & 20.98 & 441 & 349.0 & 0.791 & 52 & 5 & 0.52 & 0.04 & 3.61 & -15 & 11 & $\mathrm{H}$ \\
\hline 2533 & 19.15 & 961 & 960.2 & 0.999 & 65 & 3 & 0.90 & 0.01 & 4.15 & +34 & 3 & $\mathrm{H}$ \\
\hline 2572 & 21.40 & 441 & 397.4 & 0.901 & 56 & 6 & 0.38 & 0.05 & 2.19 & +8 & 15 & $\mathrm{H}$ \\
\hline 2574 & 22.33 & 121 & 47.8 & 0.395 & 68 & 5 & 0.45 & 0.08 & 3.18 & -14 & 13 & $\mathrm{~L}$ \\
\hline 2783 & 20.36 & 441 & 459.8 & 1.043 & 42 & 5 & 0.52 & 0.02 & 2.42 & -3 & 13 & L \\
\hline 2800 & 21.70 & 441 & 510.0 & 1.157 & 47 & 7 & 0.61 & 0.04 & 4.17 & -4 & 16 & $\mathrm{H}$ \\
\hline 2822 & 21.29 & 441 & 409.6 & 0.929 & 52 & 7 & 0.35 & 0.01 & 2.32 & -5 & 18 & $\mathrm{~L}$ \\
\hline 2946 & 22.48 & 121 & 53.9 & 0.445 & 56 & 10 & 0.28 & 0.05 & 2.01 & -15 & 27 & L \\
\hline 2958 & 20.35 & 961 & 1544.0 & 1.607 & 55 & 3 & 0.82 & 0.03 & 3.77 & +15 & 9 & $\mathrm{H}$ \\
\hline 3071 & 17.60 & 961 & 1077.2 & 1.121 & 38 & 3 & 0.55 & 0.05 & 0.96 & +35 & 7 & $\mathrm{H}$ \\
\hline 3108 & 21.57 & 441 & 426.6 & 0.967 & 61 & 5 & 0.62 & 0.05 & 3.68 & +3 & 10 & $\mathrm{H}$ \\
\hline 3131 & 21.78 & 441 & 465.7 & 1.056 & 54 & 10 & 0.37 & 0.05 & 2.75 & +3 & 18 & $\mathrm{~L}$ \\
\hline 3578 & 21.63 & 441 & 456.4 & 1.035 & 61 & 8 & 0.56 & 0.05 & 4.16 & -3 & 13 & $\mathrm{H}$ \\
\hline 3704 & 22.03 & 121 & 38.6 & 0.319 & 80 & 3 & 0.48 & 0.08 & 2.61 & +6 & 9 & $\mathrm{~L}$ \\
\hline 3730 & 22.97 & 121 & 58.3 & 0.482 & 68 & 6 & 0.45 & 0.07 & 3.57 & +2 & 15 & $\mathrm{H}$ \\
\hline 3921 & 17.39 & 2601 & 6240.7 & 2.399 & 59 & 5 & 1.42 & 0.01 & 5.14 & +12 & 3 & $\mathrm{H}$ \\
\hline 4113 & 21.22 & 441 & 372.6 & 0.845 & 69 & 4 & 0.48 & 0.06 & 2.56 & +2 & 9 & $\mathrm{~L}$ \\
\hline 4371 & 21.16 & 441 & 377.8 & 0.857 & 25 & 19 & 0.30 & 0.03 & 1.75 & -39 & 40 & $\mathrm{~L}$ \\
\hline 4376 & 22.36 & 121 & 50.7 & 0.419 & 71 & 3 & 0.42 & 0.08 & 2.24 & +1 & 11 & $\mathrm{~L}$ \\
\hline 4402 & 18.03 & 1681 & 2481.9 & 1.476 & 75 & 1 & 1.57 & 0.04 & 3.25 & +9 & 2 & $\mathrm{H}$ \\
\hline 4465 & 20.92 & 121 & 27.7 & 0.229 & 61 & 5 & 0.40 & 0.06 & 2.70 & -9 & 11 & L \\
\hline 4498 & 21.82 & 441 & 429.2 & 0.973 & 40 & 15 & 0.33 & 0.01 & 2.46 & +40 & 30 & $\mathrm{~L}$ \\
\hline 4657 & 19.31 & 961 & 1036.9 & 1.079 & 37 & 2 & 0.70 & 0.05 & 2.53 & +5 & 11 & $\mathrm{H}$ \\
\hline 4730 & 19.79 & 961 & 1144.6 & 1.191 & 32 & 6 & 0.90 & 0.09 & 6.71 & +3 & 24 & $\mathrm{~L}$ \\
\hline 4806 & 19.78 & 961 & 973.7 & 1.013 & 65 & 1 & 0.70 & 0.07 & 2.50 & -4 & 3 & $\mathrm{H}$ \\
\hline 4922 & 21.77 & 441 & 418.8 & 0.950 & 64 & 5 & 0.71 & 0.05 & 5.65 & +13 & 9 & $\mathrm{H}$ \\
\hline 5022 & 21.96 & 121 & 48.3 & 0.399 & 73 & 4 & 0.61 & 0.07 & 2.95 & -10 & 10 & $\mathrm{~L}$ \\
\hline 5140 & 21.79 & 441 & 382.9 & 0.868 & 50 & 7 & 0.60 & 0.05 & 2.51 & -5 & 15 & $\mathrm{~L}$ \\
\hline 5286 & 22.63 & 441 & 406.6 & 0.922 & 65 & 9 & 0.34 & 0.08 & 1.63 & +1 & 18 & $\mathrm{~L}$ \\
\hline 5317 & 21.39 & 441 & 556.1 & 1.261 & 53 & 4 & 0.59 & 0.05 & 4.70 & -5 & 11 & $\mathrm{~L}$ \\
\hline 5335 & 21.77 & 121 & 37.0 & 0.306 & 30 & 20 & 0.29 & 0.05 & 2.15 & +10 & 15 & $\mathrm{H}$ \\
\hline 5361 & 20.90 & 121 & 19.8 & 0.164 & 46 & 7 & 0.19 & 0.03 & 0.91 & -5 & 14 & $\mathrm{~L}$ \\
\hline 5515 & 21.33 & 121 & 56.8 & 0.470 & 49 & 8 & 0.39 & 0.04 & 3.03 & +35 & 21 & $\mathrm{H}$ \\
\hline 5565 & 21.82 & 121 & 78.4 & 0.648 & 63 & 5 & 0.43 & 0.04 & 1.55 & -8 & 10 & $\mathrm{~L}$ \\
\hline 6125 & 20.47 & 441 & 0.8 & 0.002 & 57 & 3 & 0.22 & 0.05 & 1.27 & -5 & 19 & $\mathrm{H}$ \\
\hline 6253 & 22.56 & 441 & 430.1 & 0.975 & 76 & 5 & 0.61 & 0.07 & 2.99 & +7 & 9 & $\mathrm{H}$ \\
\hline 6406 & 20.79 & 441 & 390.6 & 0.886 & 45 & 6 & 0.60 & 0.03 & 4.59 & 0 & 13 & $\mathrm{H}$ \\
\hline 6452 & 21.88 & 121 & 35.9 & 0.297 & 45 & 13 & 0.24 & 0.04 & 1.15 & -60 & 12 & $\mathrm{~L}$ \\
\hline 6568 & 20.98 & 441 & 341.5 & 0.774 & 53 & 6 & 0.46 & 0.06 & 2.68 & -15 & 13 & $\mathrm{~L}$ \\
\hline 6585 & 18.62 & 1681 & 3408.1 & 2.027 & 71 & 1 & 1.45 & 0.01 & 6.97 & -8 & 2 & $\mathrm{H}$ \\
\hline 6657 & 19.32 & 441 & 235.2 & 0.533 & 28 & 6 & 0.50 & 0.07 & 2.40 & +4 & 19 & $\mathrm{H}$ \\
\hline 6743 & 22.08 & 121 & 60.7 & 0.502 & 39 & 13 & 0.33 & 0.03 & 2.40 & +15 & 52 & $\mathrm{H}$ \\
\hline 6921 & 21.88 & 441 & 400.5 & 0.908 & 47 & 13 & 0.35 & 0.03 & 2.03 & $\begin{array}{l}+13 \\
\end{array}$ & 34 & $\mathrm{H}$ \\
\hline 7298 & 19.88 & 441 & 555.6 & 1.260 & 33 & 15 & 0.50 & 0.03 & 2.65 & +15 & 21 & $\mathrm{H}$ \\
\hline 7429 & 20.42 & 441 & 300.4 & 0.681 & 55 & 5 & 0.40 & 0.05 & 1.93 & -15 & 10 & $\mathrm{~L}$ \\
\hline 7526 & 19.46 & 441 & 409.9 & 0.930 & 59 & 1 & 0.77 & 0.08 & 3.87 & -5 & 4 & $\mathrm{H}$ \\
\hline 7597 & 22.66 & 121 & 50.4 & 0.416 & 68 & 9 & 0.26 & 0.05 & 1.42 & -13 & 19 & L \\
\hline 7725 & 21.71 & 441 & 353.4 & 0.801 & 62 & 7 & 0.49 & 0.05 & 3.15 & +15 & 9 & $\mathrm{~L}$ \\
\hline 7733 & 21.34 & 441 & 418.4 & 0.949 & 51 & 4 & 0.68 & 0.06 & 3.90 & -10 & 10 & $\mathrm{H}$ \\
\hline 7856 & 20.59 & 441 & 303.2 & 0.688 & 54 & 6 & 0.40 & 0.02 & 2.47 & +9 & 12 & $\mathrm{~L}$ \\
\hline 7866 & 20.66 & 121 & 21.4 & 0.177 & 53 & 8 & 0.30 & 0.03 & 1.08 & +27 & 19 & $\mathrm{~L}$ \\
\hline 8034 & 21.69 & 121 & 23.0 & 0.190 & 42 & 14 & 0.22 & 0.03 & 1.60 & 0 & 51 & $\mathrm{H}$ \\
\hline 8190 & 20.39 & 441 & 273.1 & 0.619 & 45 & 5 & 0.38 & 0.01 & 2.03 & -2 & 19 & $\mathrm{H}$ \\
\hline 8360 & 21.97 & 121 & 55.0 & 0.455 & 63 & 9 & 0.52 & 0.05 & 3.73 & +27 & 14 & $\mathrm{~L}$ \\
\hline 8526 & 21.46 & 441 & 394.1 & 0.894 & 71 & 5 & 0.53 & 0.03 & 3.57 & +12 & 7 & L \\
\hline
\end{tabular}


Table A.5: $K$-corrections for a Spectral Energy Distribution corresponding to type Sa, computed via synthetic photometry as described in chapter 4.6.4.

\begin{tabular}{ccccccccc}
\hline \multicolumn{1}{c}{$\begin{array}{c}B \rightarrow B_{J} \\
{[\mathrm{mag}]}\end{array}$} & $\begin{array}{c}g \rightarrow B_{J} \\
{[\mathrm{mag}]}\end{array}$ & $\begin{array}{c}R \rightarrow B_{J} \\
{[\mathrm{mag}]}\end{array}$ & $\begin{array}{c}I \rightarrow B_{J} \\
{[\mathrm{mag}]}\end{array}$ & $\begin{array}{c}B_{J} \rightarrow B_{J} \\
{[\mathrm{mag}]}\end{array}$ & $\begin{array}{c}V_{J} \rightarrow V_{J} \\
{[\mathrm{mag}]}\end{array}$ & $\begin{array}{c}R_{C} \rightarrow R_{C} \\
{[\mathrm{mag}]}\end{array}$ & $\begin{array}{c}I_{C} \rightarrow I_{C} \\
{[\mathrm{mag}]}\end{array}$ \\
\hline & & & & & & & \\
0.0 & +0.08 & -0.66 & -1.41 & -1.77 & 0.00 & 0.00 & 0.00 & 0.00 \\
0.1 & +0.48 & -0.50 & -1.36 & -1.73 & +0.37 & +0.11 & +0.06 & +0.04 \\
0.2 & +0.84 & -0.20 & -1.28 & -1.70 & +0.72 & +0.28 & +0.14 & +0.07 \\
0.3 & +1.10 & +0.16 & -1.19 & -1.68 & +1.01 & +0.55 & +0.24 & +0.09 \\
0.4 & +1.37 & +0.51 & -1.04 & -1.62 & +1.27 & +0.88 & +0.39 & +0.14 \\
0.5 & +1.66 & +0.71 & -0.86 & -1.54 & +1.55 & +1.17 & +0.59 & +0.23 \\
0.6 & +1.95 & +0.88 & -0.63 & -1.46 & +1.83 & +1.39 & +0.82 & +0.30 \\
0.7 & +2.13 & +1.16 & -0.40 & -1.35 & +2.06 & +1.57 & +1.05 & +0.41 \\
0.8 & +2.22 & +1.41 & -0.19 & -1.21 & +2.19 & +1.79 & +1.27 & +0.53 \\
0.9 & +2.25 & +1.71 & +0.01 & -0.99 & +2.25 & +2.03 & +1.47 & +0.74 \\
1.0 & +2.27 & +1.84 & +0.19 & -0.75 & +2.29 & +2.27 & +1.65 & +0.98 \\
& & & & & & & & \\
\hline
\end{tabular}

Table A.6: Same as table A.5, but for a Spectral Energy Distribution corresponding to type Sb.

\begin{tabular}{ccccccccc}
\hline \multicolumn{2}{c}{$\begin{array}{c}B \rightarrow B_{J} \\
{[\mathrm{mag}]}\end{array}$} & $\begin{array}{c}g \rightarrow B_{J} \\
{[\mathrm{mag}]}\end{array}$ & $\begin{array}{c}R \rightarrow B_{J} \\
{[\mathrm{mag}]}\end{array}$ & $\begin{array}{c}I \rightarrow B_{J} \\
{[\mathrm{mag}]}\end{array}$ & $\begin{array}{c}B_{J} \rightarrow B_{J} \\
{[\mathrm{mag}]}\end{array}$ & $\begin{array}{c}V_{J} \rightarrow V_{J} \\
{[\mathrm{mag}]}\end{array}$ & $\begin{array}{c}R_{C} \rightarrow R_{C} \\
{[\mathrm{mag}]}\end{array}$ & $\begin{array}{c}I_{C} \rightarrow I_{C} \\
{[\mathrm{mag}]}\end{array}$ \\
\hline & & & & & & & & \\
0.0 & +0.06 & -0.59 & -1.26 & -1.54 & 0.00 & 0.00 & 0.00 & 0.00 \\
0.1 & +0.42 & -0.47 & -1.23 & -1.55 & +0.32 & +0.08 & +0.03 & -0.01 \\
0.2 & +0.76 & -0.23 & -1.18 & -1.55 & +0.65 & +0.21 & +0.08 & -0.01 \\
0.3 & +1.01 & +0.09 & -1.12 & -1.55 & +0.93 & +0.43 & +0.15 & -0.01 \\
0.4 & +1.23 & +0.43 & -1.01 & -1.52 & +1.16 & +0.73 & +0.27 & +0.02 \\
0.5 & +1.46 & +0.63 & -0.85 & -1.46 & +1.38 & +1.01 & +0.44 & +0.08 \\
0.6 & +1.67 & +0.78 & -0.65 & -1.40 & +1.60 & +1.22 & +0.64 & +0.13 \\
0.7 & +1.80 & +0.99 & -0.45 & -1.31 & +1.76 & +1.38 & +0.86 & +0.21 \\
0.8 & +1.84 & +1.19 & -0.25 & -1.21 & +1.84 & +1.56 & +1.06 & +0.31 \\
0.9 & +1.83 & +1.42 & -0.07 & -1.02 & +1.86 & +1.76 & +1.24 & +0.48 \\
1.0 & +1.82 & +1.52 & +0.10 & -0.81 & +1.86 & +1.94 & +1.40 & +0.68 \\
& & & & & & & & \\
\hline
\end{tabular}


Table A.7: Same as table A.5, but for a Spectral Energy Distribution corresponding to type Sc.

\begin{tabular}{ccccccccr}
\hline \multicolumn{1}{c}{$\begin{array}{c}\text { z } \\
\text { [mag] }\end{array}$} & $\begin{array}{c}g \rightarrow B_{J} \\
{[\mathrm{mag}]}\end{array}$ & $\begin{array}{c}R \rightarrow B_{J} \\
{[\mathrm{mag}]}\end{array}$ & $\begin{array}{c}I \rightarrow B_{J} \\
{[\mathrm{mag}]}\end{array}$ & $\begin{array}{c}B_{J} \rightarrow B_{J} \\
{[\mathrm{mag}]}\end{array}$ & $\begin{array}{c}V_{J} \rightarrow V_{J} \\
{[\mathrm{mag}]}\end{array}$ & $\begin{array}{c}R_{C} \rightarrow R_{C} \\
{[\mathrm{mag}]}\end{array}$ & $\begin{array}{c}I_{C} \rightarrow I_{C} \\
{[\mathrm{mag}]}\end{array}$ \\
\hline & & & & & & & \\
0.0 & +0.04 & -0.51 & -1.09 & -1.33 & 0.00 & 0.00 & 0.00 & 0.00 \\
0.1 & +0.36 & -0.44 & -1.09 & -1.37 & +0.28 & +0.04 & 0.00 & -0.03 \\
0.2 & +0.67 & -0.27 & -1.07 & -1.39 & +0.58 & +0.13 & +0.03 & -0.05 \\
0.3 & +0.88 & +0.02 & -1.04 & -1.40 & +0.82 & +0.29 & +0.07 & -0.07 \\
0.4 & +1.03 & +0.35 & -0.97 & -1.39 & +0.99 & +0.56 & +0.14 & -0.06 \\
0.5 & +1.18 & +0.53 & -0.85 & -1.36 & +1.14 & +0.82 & +0.27 & -0.03 \\
0.6 & +1.31 & +0.63 & -0.68 & -1.33 & +1.28 & +1.01 & +0.45 & 0.00 \\
0.7 & +1.37 & +0.77 & -0.49 & -1.27 & +1.38 & +1.14 & +0.64 & +0.05 \\
0.8 & +1.37 & +0.90 & -0.31 & -1.20 & +1.40 & +1.26 & +0.82 & +0.11 \\
0.9 & +1.34 & +1.05 & -0.16 & -1.06 & +1.39 & +1.39 & +0.98 & +0.24 \\
1.0 & +1.31 & +1.10 & -0.03 & -0.87 & +1.37 & +1.51 & +1.11 & +0.42 \\
& & & & & & & & \\
\hline
\end{tabular}

Table A.8: Same as table A.5, but for a Spectral Energy Distribution corresponding to type Sdm.

\begin{tabular}{ccccccccc}
\hline \multicolumn{2}{c}{$\begin{array}{c}B \rightarrow B_{J} \\
{[\mathrm{mag}]}\end{array}$} & $\begin{array}{c}g \rightarrow B_{J} \\
{[\mathrm{mag}]}\end{array}$ & $\begin{array}{c}R \rightarrow B_{J} \\
{[\mathrm{mag}]}\end{array}$ & $\begin{array}{c}I \rightarrow B_{J} \\
{[\mathrm{mag}]}\end{array}$ & $\begin{array}{c}B_{J} \rightarrow B_{J} \\
{[\mathrm{mag}]}\end{array}$ & $\begin{array}{c}V_{J} \rightarrow V_{J} \\
{[\mathrm{mag}]}\end{array}$ & $\begin{array}{c}R_{C} \rightarrow R_{C} \\
{[\mathrm{mag}]}\end{array}$ & $\begin{array}{c}I_{C} \rightarrow I_{C} \\
{[\mathrm{mag}]}\end{array}$ \\
\hline & & & & & & & \\
\hline 0.0 & +0.03 & -0.45 & -0.96 & -1.16 & 0.00 & 0.00 & 0.00 & 0.00 \\
0.1 & +0.32 & -0.41 & -0.98 & -1.21 & +0.25 & +0.01 & -0.02 & -0.06 \\
0.2 & +0.62 & -0.29 & -0.98 & -1.25 & +0.54 & +0.06 & -0.02 & -0.10 \\
0.3 & +0.81 & -0.03 & -0.98 & -1.28 & +0.76 & +0.18 & -0.01 & -0.13 \\
0.4 & +0.92 & +0.31 & -0.93 & -1.29 & +0.91 & +0.43 & +0.03 & -0.14 \\
0.5 & +1.02 & +0.48 & -0.85 & -1.28 & +1.01 & +0.70 & +0.13 & -0.13 \\
0.6 & +1.10 & +0.55 & -0.69 & -1.27 & +1.10 & +0.88 & +0.30 & -0.13 \\
0.7 & +1.13 & +0.65 & -0.52 & -1.24 & +1.16 & +0.99 & +0.48 & -0.10 \\
0.8 & +1.12 & +0.74 & -0.35 & -1.20 & +1.16 & +1.07 & +0.64 & -0.06 \\
0.9 & +1.08 & +0.83 & -0.21 & -1.08 & +1.13 & +1.16 & +0.79 & +0.04 \\
1.0 & +1.04 & +0.86 & -0.09 & -0.92 & +1.10 & +1.24 & +0.90 & +0.20 \\
& & & & & & & & \\
\hline
\end{tabular}


Table A.9: Same as table A.5, but for a Spectral Energy Distribution corresponding to type Im.

\begin{tabular}{ccccccccc}
\hline \multicolumn{1}{c}{$z$} & $\begin{array}{c}B \rightarrow B_{J} \\
{[\mathrm{mag}]}\end{array}$ & $\begin{array}{c}g \rightarrow B_{J} \\
{[\mathrm{mag}]}\end{array}$ & $\begin{array}{c}R \rightarrow B_{J} \\
{[\mathrm{mag}]}\end{array}$ & $\begin{array}{c}I \rightarrow B_{J} \\
{[\mathrm{mag}]}\end{array}$ & $\begin{array}{c}B_{J} \rightarrow B_{J} \\
{[\mathrm{mag}]}\end{array}$ & $\begin{array}{c}V_{J} \rightarrow V_{J} \\
{[\mathrm{mag}]}\end{array}$ & $\begin{array}{c}R_{C} \rightarrow R_{C} \\
{[\mathrm{mag}]}\end{array}$ & $\begin{array}{c}I_{C} \rightarrow I_{C} \\
{[\mathrm{mag}]}\end{array}$ \\
\hline & & & & & & & \\
0.0 & +0.01 & -0.29 & -0.48 & -0.68 & 0.00 & 0.00 & 0.00 & 0.00 \\
0.1 & +0.23 & -0.34 & -0.57 & -0.78 & +0.17 & -0.07 & -0.09 & -0.11 \\
0.2 & +0.52 & -0.35 & -0.64 & -0.87 & +0.43 & -0.12 & -0.16 & -0.19 \\
0.3 & +0.69 & -0.15 & -0.70 & -0.95 & +0.63 & -0.11 & -0.22 & -0.27 \\
0.4 & +0.72 & +0.20 & -0.74 & -1.02 & +0.72 & +0.09 & -0.26 & -0.34 \\
0.5 & +0.74 & +0.38 & -0.74 & -1.07 & +0.75 & +0.35 & -0.24 & -0.40 \\
0.6 & +0.74 & +0.39 & -0.63 & -1.12 & +0.76 & +0.53 & -0.12 & -0.45 \\
0.7 & +0.73 & +0.41 & -0.48 & -1.16 & +0.76 & +0.60 & +0.03 & -0.48 \\
0.8 & +0.69 & +0.42 & -0.34 & -1.18 & +0.73 & +0.62 & +0.18 & -0.51 \\
0.9 & +0.64 & +0.44 & -0.22 & -1.15 & +0.69 & +0.64 & +0.30 & -0.50 \\
1.0 & +0.59 & +0.43 & -0.13 & -1.02 & +0.64 & +0.66 & +0.38 & -0.39 \\
& & & & & & & & \\
\hline
\end{tabular}




\section{Appendix B}

\section{Kinematic Data}

The results of the kinematic analysis described in chapter 5 are given in table B.1 for all 77 spirals with rotation curves that were appropriate for the derivation of $V_{\max }$. The respective columns have the following contents:

ID - FDF identification number as given in the photometric catalogue presented by Heidt et al. (2003).

line - Emission line used for the derivation of $V_{\max }$. This was either the $\mathrm{H} \alpha, \mathrm{H} \beta$ or [O III] 5007 line or the [O II] 3727 doublet.

qual - Quality of the corresponding rotation curve, "H" denotes the high quality data, "L" represents low quality curves, see chapter 5.2.2 for the definitions.

DIMM — Seeing during spectroscopy as measured with the DIMM telescope. Note that the given values had to be slightly corrected according to the wavelength of the emission line used for the rotation curve extraction, see chapter 5.2.2.

$V_{\max }$ - Maximum rotation velocity as derived from the RC modelling based on the emission line given in column "line", assuming a "riseturnover-flat" shape of the intrinsic RC and using a "by-eye" fit.

$\sigma_{\text {vmax }}$ - Error on $V_{\max }$, computed via error propagation as described in chapter 5.2.3.

$V_{\max \chi}$ - Maximum rotation velocity as derived from the RC modelling using a $\chi^{2}$-fit as defined in Eq. 5.17 in chapter 5.2.2.

$\sigma_{\operatorname{vmax} \chi}-$ Error on $V_{\max \chi}$, computed as de- scribed in chapter 5.2.3.

$V_{\max 2}$ - Maximum rotation velocity from $\chi^{2}$ fitting, based on a different emission line than given in column "line". This alternate derivation of $V_{\max }$ was feasible in the case of 24 spirals (see chapter 5.2.4).

$V_{2.2}$ - Rotation velocity at a radius $r=2.2 r_{\mathrm{d}}$, derived with a $\chi^{2}$-fit and an intrinsic RC as defined by Persic \& Salucci (1991).

$\sigma_{\mathrm{v} 2.2}$ - Error on $V_{2.2}$, computed as described in chapter 5.2.3.

$V_{\text {opt }}$ - Rotation velocity at a radius $r=3.2 r_{\mathrm{d}}$, derived with a $\chi^{2}$-fit and an intrinsic RC as defined by Persic et al. (1996).

$\sigma_{\text {vopt }}$ - Error on $V_{\text {opt }}$, computed as described in chapter 5.2.3.

$M_{\text {vir }}$ - Logarithmic virial mass, derived following van den Bosch (2003), see chapter 5.4.

$\sigma_{\text {Mvir }}$ - Error on the logarithmic virial mass, computed according to Eq. 5.23 in chapter 5.4. 
Table B.1: The kinematic data from the rotation curve modelling for the 77 spirals from the FORS Deep Field sample with rotation curves which were appropriate for the derivation of $V_{\max }$.

\begin{tabular}{|c|c|c|c|c|c|c|c|c|c|c|c|c|c|c|}
\hline ID & line & qual & $\begin{array}{c}\text { DIMM } \\
{[\operatorname{arcsec}]}\end{array}$ & $\begin{array}{r}V_{\max } \\
{[\mathrm{km} / \mathrm{s}]}\end{array}$ & $\begin{array}{l}\sigma_{\mathrm{vmax}} \\
{[\mathrm{km} / \mathrm{s}]}\end{array}$ & $\begin{array}{l}V_{\max } \chi \\
{[\mathrm{km} / \mathrm{s}]}\end{array}$ & $\begin{array}{r}\sigma_{\mathrm{vmax}} \\
{[\mathrm{km} / \mathrm{s}]}\end{array}$ & $\begin{array}{l}V_{\max 2} \\
{[\mathrm{~km} / \mathrm{s}]}\end{array}$ & $\begin{array}{r}V_{2} .2 \\
{[\mathrm{~km} / \mathrm{s}]}\end{array}$ & $\begin{array}{r}\sigma_{\mathrm{v} 2} .2 \\
{[\mathrm{~km} / \mathrm{s}]}\end{array}$ & $\begin{array}{r}V_{\mathrm{opt}} \\
{[\mathrm{km} / \mathrm{s}]}\end{array}$ & $\begin{array}{r}\sigma_{\mathrm{vopt}} \\
{[\mathrm{km} / \mathrm{s}]}\end{array}$ & $\begin{array}{l}M_{\mathrm{vir}} \\
{\left[M_{\odot}\right]}\end{array}$ & $\begin{array}{c}\sigma_{\mathrm{Mvir}} \\
{\left[M_{\odot}\right]}\end{array}$ \\
\hline 400 & $\mathrm{H} \beta$ & $\mathrm{L}$ & 0.80 & 74 & 19 & 74 & 19 & 74 & 74 & 18 & 74 & 17 & 10.75 & 10.47 \\
\hline 745 & $\mathrm{H} \beta$ & $\mathrm{L}$ & 0.76 & 402 & 167 & 363 & 153 & 275 & 363 & 152 & 363 & 152 & 12.15 & 12.07 \\
\hline 870 & [OIII] & $\mathrm{H}$ & 0.66 & 96 & 6 & 101 & 6 & 98 & 106 & 9 & 111 & 7 & 10.72 & 9.84 \\
\hline 1082 & {$[\mathrm{OII}]$} & $\mathrm{H}$ & 0.43 & 118 & 16 & 108 & 15 & & 108 & 15 & 113 & 14 & 11.01 & 10.46 \\
\hline 1224 & [OII $]$ & $\mathrm{L}$ & 0.43 & 43 & 16 & 43 & 16 & & 43 & 15 & 48 & 17 & 9.95 & 9.81 \\
\hline 1327 & [OIII] & $\mathrm{L}$ & 0.76 & 204 & 36 & 228 & 39 & 170 & 222 & 37 & 234 & 39 & 11.46 & 11.02 \\
\hline 1449 & $\mathrm{H} \alpha$ & $\mathrm{L}$ & 0.66 & 51 & 16 & 39 & 15 & & 41 & 38 & 46 & 42 & 9.98 & 9.77 \\
\hline 1476 & [OII $]$ & $\mathrm{L}$ & 0.51 & 283 & 26 & 278 & 26 & & 278 & 26 & 298 & 29 & 11.96 & 11.30 \\
\hline 1569 & [OII] & $\mathrm{L}$ & 0.43 & 57 & 50 & 44 & 47 & & 48 & 41 & 53 & 45 & 10.24 & 10.46 \\
\hline 1625 & [OII $]$ & $\mathrm{L}$ & 0.66 & 122 & 42 & 127 & 42 & 125 & 127 & 41 & 142 & 45 & 10.75 & 10.56 \\
\hline 1655 & [OIII] & $\mathrm{L}$ & 0.81 & 126 & 8 & 121 & 8 & 155 & 121 & 8 & 130 & 10 & 10.97 & 10.15 \\
\hline 1699 & $\mathrm{H} \beta$ & $\mathrm{L}$ & 0.81 & 55 & 15 & 55 & 15 & 42 & 55 & 17 & 58 & 17 & 9.78 & 9.54 \\
\hline 1834 & [OIII] & $\mathrm{L}$ & 0.82 & 62 & 37 & 57 & 37 & & 57 & 38 & 63 & 42 & 10.08 & 10.16 \\
\hline 1928 & {$[\mathrm{OII}]$} & $\mathrm{L}$ & 0.82 & 105 & 29 & 76 & 28 & & 76 & 29 & 80 & 31 & 10.78 & 10.53 \\
\hline 2007 & [OII] & $\mathrm{L}$ & 0.81 & 84 & 20 & 93 & 21 & & 93 & 21 & 100 & 24 & 10.71 & 10.42 \\
\hline 2067 & [OII $]$ & $\mathrm{H}$ & 0.76 & 210 & 58 & 210 & 58 & & 205 & 57 & 205 & 57 & 11.53 & 11.28 \\
\hline 2174 & [OII] & $\mathrm{H}$ & 0.51 & 175 & 24 & 175 & 24 & & 170 & 22 & 175 & 24 & 11.37 & 10.83 \\
\hline 2246 & [OII] & $\mathrm{H}$ & 0.51 & 183 & 29 & 178 & 29 & & 178 & 31 & 174 & 29 & 11.55 & 11.06 \\
\hline 2328 & [OII] & $\mathrm{L}$ & 0.80 & 150 & 18 & 291 & 20 & & 285 & 20 & 301 & 20 & 11.19 & 10.61 \\
\hline 2341 & [OII] & $\mathrm{H}$ & 0.74 & 279 & 107 & 258 & 99 & & 258 & 101 & 258 & 100 & 11.71 & 11.60 \\
\hline 2353 & [OII $]$ & $\mathrm{H}$ & 0.66 & 209 & 44 & 219 & 45 & & 214 & 43 & 214 & 43 & 11.61 & 11.26 \\
\hline 2397 & [OII] & $\mathrm{H}$ & 0.81 & 98 & 22 & 71 & 20 & 74 & 71 & 21 & 71 & 18 & 11.05 & 10.72 \\
\hline 2484 & [OII] & $\mathrm{H}$ & 0.80 & 169 & 36 & 159 & 36 & & 159 & 38 & 164 & 40 & 11.42 & 11.06 \\
\hline 2533 & [OII $]$ & $\mathrm{H}$ & 0.66 & 230 & 16 & 244 & 16 & 240 & 239 & 16 & 239 & 14 & 11.74 & 10.88 \\
\hline 2572 & [OII] & $\mathrm{H}$ & 0.66 & 74 & 19 & 57 & 19 & & 58 & 18 & 58 & 17 & 10.51 & 10.21 \\
\hline 2574 & {$[\mathrm{OII}]$} & $\mathrm{L}$ & 0.76 & 162 & 29 & 176 & 29 & & 176 & 31 & 186 & 32 & 11.32 & 10.93 \\
\hline 2783 & $\mathrm{H} \beta$ & $\mathrm{L}$ & 0.66 & 84 & 17 & 65 & 16 & 37 & 66 & 17 & 66 & 15 & 10.64 & 10.25 \\
\hline 2800 & [OII] & $\mathrm{H}$ & 0.66 & 143 & 27 & 156 & 28 & & 160 & 32 & 174 & 34 & 11.33 & 10.92 \\
\hline 2822 & [OII $]$ & $\mathrm{L}$ & 0.74 & 107 & 21 & 80 & 20 & & 78 & 21 & 81 & 22 & 10.83 & 10.42 \\
\hline 2946 & [OII $]$ & $\mathrm{L}$ & 0.43 & 108 & 34 & 63 & 30 & & 60 & 25 & 60 & 10 & 10.78 & 10.59 \\
\hline 2958 & [OIII] & $\mathrm{H}$ & 0.74 & 141 & 13 & 89 & 11 & 130 & 89 & 9 & 99 & 12 & 11.27 & 10.55 \\
\hline 3071 & $\mathrm{H} \beta$ & $\mathrm{H}$ & 0.81 & 176 & 20 & 130 & 16 & 110 & 136 & 17 & 147 & 17 & 10.88 & 10.27 \\
\hline 3108 & [OII] & $\mathrm{H}$ & 0.51 & 104 & 26 & 99 & 25 & & 104 & 27 & 113 & 29 & 11.01 & 10.70 \\
\hline 3131 & [OII] & $\mathrm{L}$ & 0.89 & 166 & 26 & 155 & 25 & & 155 & 27 & 155 & 25 & 11.29 & 10.82 \\
\hline 3578 & [OII $]$ & $\mathrm{H}$ & 0.89 & 154 & 37 & 213 & 39 & & 208 & 36 & 213 & 37 & 11.39 & 11.09 \\
\hline 3704 & [OIII] & $\mathrm{L}$ & 0.74 & 40 & 20 & 36 & 20 & & 35 & 19 & 38 & 21 & 10.04 & 10.03 \\
\hline 3730 & {$[\mathrm{OII}]$} & $\mathrm{H}$ & 0.89 & 156 & 41 & 145 & 40 & & 140 & 37 & 145 & 40 & 11.34 & 11.08 \\
\hline 3921 & [OII $]$ & $\mathrm{H}$ & 0.80 & 245 & 19 & 235 & 19 & 320 & 221 & 16 & 235 & 19 & 11.89 & 11.09 \\
\hline 4113 & [OII] & $\mathrm{L}$ & 0.80 & 249 & 53 & 305 & 54 & & 310 & 55 & 336 & 59 & 11.62 & 11.26 \\
\hline 4371 & $\mathrm{H} \beta$ & $\mathrm{L}$ & 0.76 & 361 & 330 & 329 & 301 & & 329 & 301 & 345 & 316 & 11.76 & 12.03 \\
\hline 4376 & $\mathrm{H} \beta$ & $\mathrm{L}$ & 0.66 & 74 & 18 & 69 & 18 & 48 & 74 & 21 & 79 & 22 & 10.52 & 10.21 \\
\hline 4402 & $\mathrm{H} \alpha$ & $\mathrm{H}$ & 0.51 & 95 & 3 & 90 & 3 & & 90 & 3 & 90 & 3 & 10.87 & 9.71 \\
\hline 4465 & [OII] & $\mathrm{L}$ & 0.81 & 170 & 26 & 165 & 26 & & 165 & 29 & 170 & 30 & 11.30 & 10.83 \\
\hline 4498 & [OII $]$ & $\mathrm{L}$ & 0.51 & 95 & 58 & 46 & 37 & & 45 & 37 & 45 & 37 & 10.74 & 10.84 \\
\hline 4657 & $\mathrm{H} \beta$ & $\mathrm{H}$ & 0.81 & 212 & 14 & 226 & 14 & 165 & 233 & 16 & 253 & 17 & 11.46 & 10.63 \\
\hline 4730 & [OII] & $\mathrm{L}$ & 0.43 & 430 & 84 & 381 & 77 & & 365 & 72 & 349 & 71 & 12.50 & 12.10 \\
\hline 4806 & $\mathrm{H} \beta$ & $\mathrm{H}$ & 0.74 & 209 & 17 & 199 & 17 & & 209 & 19 & 230 & 18 & 11.44 & 10.73 \\
\hline 4922 & [OII] & $\mathrm{H}$ & 0.43 & 156 & 27 & 152 & 27 & & 143 & 23 & 133 & 23 & 11.54 & 11.10 \\
\hline 5022 & [OIII] & $\mathrm{L}$ & 0.80 & 75 & 6 & 50 & 5 & 43 & 55 & 8 & 58 & 7 & 10.61 & 9.90 \\
\hline 5140 & $\mathrm{H} \beta$ & $\mathrm{L}$ & 0.82 & 125 & 39 & 114 & 39 & & 125 & 43 & 141 & 46 & 11.00 & 10.80 \\
\hline 5286 & [OIII] & $\mathrm{L}$ & 0.66 & 23 & 7 & 20 & 7 & & 23 & 10 & 24 & 10 & 9.35 & 9.16 \\
\hline 5317 & {$[\mathrm{OII}]$} & $\mathrm{L}$ & 0.81 & 236 & 36 & 144 & 35 & & 135 & 33 & 135 & 32 & 11.83 & 11.33 \\
\hline 5335 & [OII] & $\mathrm{H}$ & 0.43 & 220 & 135 & 118 & 75 & & 117 & 74 & 124 & 79 & 11.44 & 11.51 \\
\hline 5361 & $\mathrm{H} \beta$ & $\mathrm{L}$ & 0.89 & 75 & 19 & 45 & 18 & & 40 & 16 & 46 & 19 & 10.11 & 9.84 \\
\hline 5515 & [OII] & $\mathrm{H}$ & 0.43 & 269 & 87 & 253 & 83 & & 237 & 76 & 242 & 79 & 11.76 & 11.56 \\
\hline 5565 & [OIII] & $\mathrm{L}$ & 0.81 & 41 & 10 & 43 & 10 & & 41 & 10 & 47 & 13 & 9.81 & 9.52 \\
\hline 6125 & {$[\mathrm{OII}]$} & $\mathrm{H}$ & 0.43 & 152 & 18 & 142 & 18 & 130 & 127 & 13 & 137 & 17 & 10.87 & 10.39 \\
\hline 6253 & [OIII] & $\mathrm{H}$ & 0.80 & 95 & 19 & 110 & 19 & & 114 & 18 & 134 & 22 & 10.83 & 10.46 \\
\hline 6406 & {$[\mathrm{OII}]$} & $\mathrm{H}$ & 0.51 & 250 & 36 & 240 & 35 & & 225 & 33 & 225 & 31 & 11.86 & 11.32 \\
\hline 6452 & [OIII] & $\mathrm{L}$ & 0.51 & 109 & 52 & 107 & 52 & & 114 & 55 & 131 & 63 & 10.56 & 10.53 \\
\hline 6568 & {$[\mathrm{OII}]$} & $\mathrm{L}$ & 0.80 & 144 & 30 & 135 & 30 & & 135 & 28 & 144 & 29 & 11.17 & 10.80 \\
\hline 6585 & [OIII] & $\mathrm{H}$ & 0.43 & 295 & 7 & 329 & 7 & 200 & 324 & 10 & 319 & 9 & 12.20 & 10.89 \\
\hline 6657 & $\mathrm{H} \beta$ & $\mathrm{H}$ & 0.82 & 254 & 54 & 281 & 59 & 210 & 281 & 60 & 286 & 60 & 11.59 & 11.25 \\
\hline 6743 & [OII] & $\mathrm{H}$ & 0.74 & 118 & 48 & 108 & 45 & & 108 & 45 & 113 & 46 & 10.92 & 10.84 \\
\hline 6921 & $\mathrm{H} \beta$ & $\mathrm{H}$ & 0.66 & 111 & 46 & 113 & 46 & & 116 & 47 & 129 & 53 & 10.81 & 10.72 \\
\hline 7298 & [OIII] & $\mathrm{H}$ & 0.74 & 115 & 56 & 78 & 44 & & 75 & 41 & 80 & 44 & 10.95 & 10.94 \\
\hline 7429 & $\mathrm{H} \beta$ & $\mathrm{L}$ & 0.80 & 90 & 17 & 85 & 16 & 82 & 85 & 16 & 92 & 18 & 10.60 & 10.19 \\
\hline 7526 & $\mathrm{H} \beta$ & $\mathrm{H}$ & 0.51 & 181 & 7 & 185 & 7 & 170 & 181 & 7 & 190 & 8 & 11.50 & 10.63 \\
\hline 7597 & [OIII] & $\mathrm{L}$ & 0.89 & 79 & 34 & 50 & 33 & & 45 & 28 & 50 & 32 & 10.36 & 10.30 \\
\hline 7725 & [OIII] & $\mathrm{L}$ & 0.66 & 84 & 38 & 74 & 37 & 125 & 74 & 37 & 74 & 36 & 10.75 & 10.70 \\
\hline 7733 & [OIII] & $\mathrm{H}$ & 0.51 & 89 & 17 & 73 & 17 & 77 & 76 & 19 & 84 & 22 & 10.90 & 10.49 \\
\hline 7856 & {$[\mathrm{OII}]$} & $\mathrm{L}$ & 0.80 & 273 & 35 & 256 & 34 & & 251 & 32 & 262 & 35 & 11.67 & 11.09 \\
\hline 7866 & $\mathrm{H} \beta$ & $\mathrm{L}$ & 0.74 & 50 & 45 & 55 & 45 & & 55 & 45 & 60 & 49 & 9.84 & 10.09 \\
\hline 8034 & {$[\mathrm{OII}]$} & $\mathrm{H}$ & 0.80 & 173 & 49 & 178 & 51 & & 173 & 49 & 178 & 51 & 11.09 & 10.85 \\
\hline 8190 & $\mathrm{H} \beta$ & $\mathrm{H}$ & 0.74 & 113 & 12 & 118 & 13 & 115 & 118 & 13 & 124 & 15 & 10.84 & 10.15 \\
\hline 8360 & [OII] & $\mathrm{L}$ & 0.89 & 120 & 62 & 125 & 62 & & 120 & 58 & 130 & 65 & 11.12 & 11.15 \\
\hline 8526 & [OIII] & $\mathrm{L}$ & 0.82 & 135 & 10 & 90 & 10 & & 90 & 11 & 92 & 10 & 11.21 & 10.44 \\
\hline
\end{tabular}




\section{Appendix $\mathrm{C}$}

\section{Data on the Scaling Relations}

The results from the analysis of the distant scaling relations in chapter 6 are given in table C.1. The respective columns have the following contents:

ID - FDF identification number as given in the photometric catalogue presented by Heidt et al. (2003).

qual - Quality of the corresponding rotation curve, "H" denotes the high quality data, "L" represents low quality curves, see chapter 5.2.2 for the definitions.

$\Delta M_{B}^{\mathrm{pt} 92}$ - Offsets of the FDF spirals from the local Tully-Fisher Relation given by Pierce \& Tully (1992).

$\sigma_{\Delta T F}-$ Errors on the offsets $\Delta M_{B}^{\mathrm{pt} 92}$, computed according to Eq. 6.10 in chapter 6.2.

$\Delta \log r_{\mathrm{d}}$ - Offsets of the FDF spirals from the local velocity-size relation of the Haynes et al. (1999) sample.

$\sigma_{\Delta V S}-$ Errors on the offsets $\Delta \log r_{\mathrm{d}}$, computed according to Eq. 6.23 in chapter 6.3.

$m_{\text {bias }}$ - Incompleteness bias, derived following Giovanelli et al. (1997).

$(B-R)$ - Rest-frame $B-R$ color index in the Johnson-Cousins standard filter system, computed from aperture magnitudes as described in chapter 6.4.2.

$D_{\mathrm{nn}}$ - Projected distance to the nearest neighboring galaxy with a relative line-of-sight velocity of $\Delta V_{\text {sys }} \leq 250 \mathrm{~km} / \mathrm{s}$. In the case of $14 \mathrm{spi}$ rals, no neighbor was found within these limits in the field-of-view of the FDF, see chapter 6.4.3. $\psi$ - Star Formation Rate as calculated from the [O II] equivalent width following Kennicutt (1992b), see chapter 6.4.4.

$A_{B}^{i, V}$ - Intrinsic $B$-band absorption following Tully et al. (1998), see chapter 4.6 .5 for the definition.

$M_{B 2}$ - Absolute $B$-band magnitude computed using the Tully et al. convention for intrinsic absorption correction.

$\Delta M_{B 2}^{\text {ver01 }}$ - Offsets of the FDF spirals from the local Tully-Fisher Relation as given by Verheijen (2001), see chapter 6.4.6 for details. 
Table C.1: Data from the analysis of the distant scaling relations as described in chapter 6 .

\begin{tabular}{|c|c|c|c|c|c|c|c|c|c|c|c|c|}
\hline ID & qual & $\begin{array}{c}\Delta M_{B}^{\mathrm{pt} 92} \\
{[\mathrm{mag}]}\end{array}$ & $\begin{array}{r}\sigma_{\Delta T F} \\
{[\mathrm{mag}]}\end{array}$ & $\underset{[\mathrm{kpc}]}{\Delta \log r_{\mathrm{d}}}$ & $\begin{array}{c}\sigma_{\Delta V S} \\
{[\mathrm{kpc}]}\end{array}$ & $\begin{array}{l}m_{\text {bias }} \\
{[\mathrm{mag}]}\end{array}$ & $\begin{array}{c}(B-R) \\
{[\mathrm{mag}]}\end{array}$ & $\begin{array}{c}D_{\mathrm{nn}} \\
{[\mathrm{kpc}]}\end{array}$ & $\begin{array}{c}\psi \\
{\left[M_{\odot} / \mathrm{yr}\right]}\end{array}$ & $\begin{array}{l}A_{B}^{i, V} \\
{[\mathrm{mag}]}\end{array}$ & $\begin{array}{l}M_{B 2} \\
{[\mathrm{mag}]}\end{array}$ & $\begin{array}{c}\Delta M_{B 2}^{\text {ver01 }} \\
{[\mathrm{mag}]}\end{array}$ \\
\hline 400 & $\mathrm{~L}$ & -2.65 & 0.87 & -0.492 & 0.157 & -1.19 & 0.21 & 220 & 3.3 & 0.18 & -19.84 & -2.63 \\
\hline 745 & $\mathrm{~L}$ & +1.02 & 1.44 & +0.566 & 0.126 & +0.05 & 1.00 & 1392 & 9.6 & 0.33 & -21.95 & +1.21 \\
\hline 870 & $\mathrm{H}$ & -2.04 & 0.21 & -0.082 & 0.033 & -0.86 & 0.73 & 177 & 3.8 & 0.20 & -20.18 & -2.04 \\
\hline 1082 & $\mathrm{H}$ & -1.63 & 0.44 & -0.069 & 0.083 & -0.63 & 0.75 & 219 & 4.9 & 0.21 & -20.48 & -1.61 \\
\hline 1224 & $\mathrm{~L}$ & -3.29 & 1.25 & -0.465 & 0.208 & -2.04 & 0.26 & 73 & 3.3 & 0.00 & -18.64 & -3.34 \\
\hline 1327 & $\mathrm{~L}$ & -0.26 & 0.58 & +0.253 & 0.062 & -0.19 & 0.91 & 386 & & 0.12 & -20.86 & -0.08 \\
\hline 1449 & $\mathrm{~L}$ & -2.61 & 1.08 & -0.240 & 0.218 & -1.75 & 0.67 & 295 & & 0.02 & -18.59 & -2.69 \\
\hline 1476 & $\mathrm{~L}$ & +1.28 & 0.32 & +0.244 & 0.073 & -0.04 & 1.52 & & 0.8 & 1.19 & -20.92 & +1.01 \\
\hline 1569 & $\mathrm{~L}$ & -2.01 & 4.27 & -0.334 & 0.993 & -1.56 & 0.69 & & 0.8 & 0.03 & -18.39 & -2.07 \\
\hline 1625 & $\mathrm{~L}$ & +0.94 & 1.16 & +0.263 & 0.192 & -0.60 & 0.84 & 52 & 0.2 & 0.31 & -18.05 & +0.94 \\
\hline 1655 & $\mathrm{~L}$ & -0.73 & 0.22 & +0.051 & 0.050 & -0.57 & 0.84 & 574 & & 0.24 & -19.79 & -0.71 \\
\hline 1699 & $\mathrm{~L}$ & -2.23 & 0.91 & +0.052 & 0.115 & -1.63 & 0.11 & 52 & & 0.05 & -18.43 & -2.27 \\
\hline 1834 & $\mathrm{~L}$ & -0.94 & 2.25 & -0.077 & 0.512 & -1.44 & 1.39 & & 0.2 & 0.08 & -17.56 & -0.96 \\
\hline 1928 & $\mathrm{~L}$ & -1.77 & 0.94 & -0.004 & 0.212 & -0.77 & 0.42 & 60 & 6.7 & 0.30 & -20.20 & -1.78 \\
\hline 2007 & $\mathrm{~L}$ & -2.73 & 0.82 & -0.276 & 0.141 & -1.03 & 0.48 & 60 & 6.7 & 0.24 & -20.35 & -2.71 \\
\hline 2067 & $\mathrm{H}$ & -0.76 & 0.93 & +0.248 & 0.067 & -0.18 & 0.97 & 1713 & 9.4 & 0.16 & -21.48 & -0.60 \\
\hline 2174 & $\mathrm{H}$ & -0.70 & 0.46 & +0.135 & 0.082 & -0.30 & 1.21 & 294 & 3.3 & 0.41 & -20.95 & -0.71 \\
\hline 2246 & $\mathrm{H}$ & -0.83 & 0.53 & +0.024 & 0.059 & -0.27 & 1.03 & & 3.8 & 0.25 & -21.14 & -0.76 \\
\hline 2328 & $\mathrm{~L}$ & -0.35 & 0.40 & +0.103 & 0.063 & -0.41 & 0.78 & 663 & 3.7 & 0.57 & -20.12 & -0.41 \\
\hline 2341 & $\mathrm{H}$ & +0.56 & 1.31 & +0.480 & 0.083 & -0.04 & 1.07 & 51 & 7.8 & 0.15 & -21.09 & +0.79 \\
\hline 2353 & $\mathrm{H}$ & -0.61 & 0.69 & +0.151 & 0.094 & -0.18 & 1.21 & & 7.9 & 0.22 & -21.35 & -0.49 \\
\hline 2397 & $\mathrm{H}$ & -2.43 & 0.76 & -0.386 & 0.159 & -0.84 & 0.71 & 916 & 5.0 & 0.11 & -20.61 & -2.41 \\
\hline 2484 & $\mathrm{H}$ & -0.88 & 0.71 & +0.041 & 0.135 & -0.32 & 1.10 & 27 & 1.8 & 0.32 & -20.98 & -0.86 \\
\hline 2533 & $\mathrm{H}$ & -0.14 & 0.24 & +0.159 & 0.029 & -0.13 & 0.99 & 174 & 6.2 & 0.68 & -21.44 & -0.25 \\
\hline 2572 & $\mathrm{H}$ & -2.39 & 0.86 & -0.226 & 0.207 & -1.19 & 0.86 & 1173 & 1.8 & 0.15 & -19.61 & -2.39 \\
\hline 2574 & $\mathrm{~L}$ & -0.18 & 0.61 & +0.069 & 0.118 & -0.36 & 0.95 & 1105 & 3.7 & 0.60 & -20.21 & -0.25 \\
\hline 2783 & $\mathrm{~L}$ & -2.03 & 0.67 & -0.194 & 0.165 & -1.02 & 0.83 & 173 & & 0.10 & -19.70 & -2.03 \\
\hline 2800 & $\mathrm{H}$ & -0.79 & 0.64 & -0.122 & 0.079 & -0.46 & 0.86 & & 4.0 & 0.23 & -20.27 & -0.75 \\
\hline 2822 & $\mathrm{~L}$ & -1.91 & 0.65 & -0.034 & 0.135 & -0.74 & 0.85 & & 4.7 & 0.22 & -20.42 & -1.91 \\
\hline 2946 & $\mathrm{~L}$ & -1.64 & 1.05 & +0.033 & 0.307 & -0.73 & 0.35 & & 5.9 & 0.26 & -20.20 & -1.64 \\
\hline 2958 & $\mathrm{H}$ & -0.52 & 0.30 & -0.084 & 0.064 & -0.47 & 0.73 & 386 & 3.0 & 0.32 & -20.01 & -0.52 \\
\hline 3071 & $\mathrm{H}$ & +0.92 & 0.39 & +0.638 & 0.053 & -0.29 & 0.83 & 107 & & 0.17 & -19.22 & +1.03 \\
\hline 3108 & $\mathrm{H}$ & -1.21 & 0.82 & -0.254 & 0.147 & -0.78 & 1.01 & 1102 & 0.8 & 0.31 & -19.61 & -1.21 \\
\hline 3131 & $\mathrm{~L}$ & -0.92 & 0.53 & +0.149 & 0.086 & -0.33 & 0.92 & 1002 & 9.7 & 0.35 & -20.97 & -0.91 \\
\hline 3578 & $\mathrm{H}$ & -1.04 & 0.81 & -0.077 & 0.104 & -0.40 & 1.06 & 879 & 3.1 & 0.44 & -20.85 & -1.07 \\
\hline 3704 & $\mathrm{~L}$ & -3.76 & 1.79 & -0.664 & 0.388 & -2.17 & 1.27 & 1583 & & 0.00 & -18.30 & -3.26 \\
\hline 3730 & $\mathrm{H}$ & -0.80 & 0.88 & -0.003 & 0.185 & -0.39 & 0.73 & & 8.3 & 0.59 & -20.69 & -0.87 \\
\hline 3921 & $\mathrm{H}$ & -0.34 & 0.28 & +0.105 & 0.034 & -0.10 & 1.01 & 135 & 3.6 & 0.55 & -21.80 & -0.38 \\
\hline 4113 & $\mathrm{~L}$ & +1.68 & 0.72 & +0.417 & 0.150 & -0.09 & 1.12 & 56 & 0.5 & 0.83 & -19.97 & +1.52 \\
\hline 4371 & $\mathrm{~L}$ & +2.85 & 5.03 & +0.800 & 0.110 & +0.03 & 1.16 & 548 & 0.6 & 0.10 & -19.61 & +3.18 \\
\hline 4376 & $\mathrm{~L}$ & -1.45 & 0.82 & -0.240 & 0.176 & -1.20 & 0.62 & 396 & 1.1 & 0.28 & -18.54 & -1.35 \\
\hline 4402 & $\mathrm{H}$ & -1.39 & 0.14 & -0.251 & 0.022 & -0.88 & 0.75 & 294 & & 0.47 & -19.39 & -1.30 \\
\hline 4465 & $\mathrm{~L}$ & -0.93 & 0.51 & +0.169 & 0.104 & -0.32 & 1.04 & 17 & 4.5 & 0.47 & -21.10 & -0.96 \\
\hline 4498 & $\mathrm{~L}$ & -2.54 & 2.33 & -0.131 & 0.427 & -0.88 & 0.82 & 108 & 6.2 & 0.10 & -20.61 & -2.52 \\
\hline 4657 & $\mathrm{H}$ & +1.34 & 0.23 & +0.328 & 0.045 & -0.17 & 0.91 & 67 & & 0.18 & -19.43 & +1.49 \\
\hline 4730 & $\mathrm{~L}$ & +0.59 & 0.65 & +0.318 & 0.082 & +0.05 & 1.41 & 108 & 3.7 & 0.19 & -22.50 & +0.90 \\
\hline 4806 & $\mathrm{H}$ & +1.38 & 0.28 & +0.325 & 0.067 & -0.18 & 0.72 & & & 0.64 & -19.58 & +1.29 \\
\hline 4922 & $\mathrm{H}$ & -1.85 & 0.59 & -0.200 & 0.098 & -0.38 & 0.22 & 235 & 18.4 & 0.50 & -21.74 & -1.89 \\
\hline 5022 & $\mathrm{~L}$ & -1.53 & 0.28 & -0.351 & 0.077 & -1.18 & 0.51 & 283 & & 0.31 & -18.64 & -1.40 \\
\hline 5140 & $\mathrm{~L}$ & +1.03 & 1.06 & +0.020 & 0.208 & -0.58 & 0.85 & 724 & & 0.23 & -17.99 & +1.05 \\
\hline 5286 & $\mathrm{~L}$ & -4.33 & 1.07 & -0.788 & 0.238 & -3.31 & 0.64 & 50 & & 0.00 & -17.46 & -4.38 \\
\hline 5317 & $\mathrm{~L}$ & -0.91 & 0.51 & +0.121 & 0.140 & -0.11 & 0.71 & 1000 & 16.5 & 0.42 & -22.18 & -0.89 \\
\hline 5335 & $\mathrm{H}$ & +0.17 & 2.33 & +0.418 & 0.166 & -0.15 & 0.92 & 880 & 6.2 & 0.12 & -20.67 & +0.37 \\
\hline 5361 & $\mathrm{~L}$ & -1.78 & 0.86 & +0.162 & 0.243 & -1.18 & 1.05 & 50 & & 0.10 & -19.05 & -1.79 \\
\hline 5515 & $\mathrm{H}$ & -0.16 & 1.09 & +0.389 & 0.113 & -0.06 & 0.81 & & 2.6 & 0.38 & -21.85 & -0.09 \\
\hline 5565 & $\mathrm{~L}$ & -2.46 & 0.84 & -0.430 & 0.155 & -2.15 & 0.25 & 547 & & 0.00 & -17.48 & -2.38 \\
\hline 6125 & $\mathrm{H}$ & -0.31 & 0.40 & +0.432 & 0.122 & -0.41 & 1.52 & 858 & 1.6 & 0.37 & -20.06 & -0.32 \\
\hline 6253 & $\mathrm{H}$ & -0.25 & 0.69 & -0.213 & 0.110 & -0.87 & 0.69 & & & 0.49 & -18.25 & -0.15 \\
\hline 6406 & $\mathrm{H}$ & -0.79 & 0.47 & +0.165 & 0.063 & -0.09 & 1.15 & 1573 & 6.8 & 0.30 & -22.18 & -0.69 \\
\hline 6452 & $\mathrm{~L}$ & +0.11 & 1.71 & +0.276 & 0.168 & -0.73 & 0.78 & 180 & & 0.16 & -18.42 & +0.14 \\
\hline 6568 & $\mathrm{~L}$ & -0.43 & 0.70 & +0.077 & 0.128 & -0.45 & 1.06 & 180 & 1.4 & 0.30 & -19.99 & -0.42 \\
\hline 6585 & $\mathrm{H}$ & +0.16 & 0.11 & +0.080 & 0.013 & -0.02 & 1.46 & 345 & & 0.98 & -22.13 & -0.06 \\
\hline 6657 & $\mathrm{H}$ & +0.73 & 0.71 & +0.457 & 0.076 & -0.08 & 1.26 & 768 & & 0.11 & -20.59 & +0.96 \\
\hline 6743 & $\mathrm{H}$ & -1.45 & 1.41 & +0.005 & 0.115 & -0.64 & 0.77 & 780 & 5.4 & 0.13 & -20.24 & -1.40 \\
\hline 6921 & $\mathrm{H}$ & -0.57 & 1.42 & +0.046 & 0.178 & -0.70 & 0.86 & 656 & & 0.18 & -19.20 & -0.55 \\
\hline 7298 & $\mathrm{H}$ & -1.87 & 1.74 & -0.051 & 0.233 & -0.66 & 0.98 & 1454 & & 0.09 & -20.58 & -1.82 \\
\hline 7429 & $\mathrm{~L}$ & -2.05 & 0.61 & -0.057 & 0.118 & -0.94 & 0.73 & 127 & 2.3 & 0.20 & -19.95 & -2.05 \\
\hline 7526 & $\mathrm{H}$ & -0.87 & 0.15 & +0.050 & 0.050 & -0.27 & 0.93 & & & 0.46 & -21.26 & -0.90 \\
\hline 7597 & $\mathrm{~L}$ & -0.79 & 1.50 & +0.004 & 0.473 & -1.10 & 0.87 & 103 & & 0.28 & -18.19 & -0.73 \\
\hline 7725 & $\mathrm{~L}$ & -2.22 & 1.58 & -0.313 & 0.320 & -1.03 & 0.86 & 351 & & 0.24 & -19.84 & -2.21 \\
\hline 7733 & $\mathrm{H}$ & -1.97 & 0.64 & -0.372 & 0.118 & -0.96 & 0.75 & 1287 & & 0.16 & -19.81 & -1.97 \\
\hline 7856 & $\mathrm{~L}$ & +1.05 & 0.43 & +0.486 & 0.071 & -0.05 & 1.23 & 359 & 2.0 & 0.48 & -20.74 & +1.06 \\
\hline 7866 & $\mathrm{~L}$ & -2.21 & 4.82 & -0.150 & 0.646 & -1.79 & 0.97 & 447 & & 0.04 & -18.06 & -2.22 \\
\hline 8034 & $\mathrm{H}$ & -0.63 & 0.96 & +0.407 & 0.078 & -0.31 & 0.90 & 780 & 5.6 & 0.20 & -20.75 & -0.55 \\
\hline 8190 & $\mathrm{H}$ & -1.67 & 0.36 & +0.054 & 0.039 & -0.68 & 0.76 & 1120 & & 0.17 & -20.34 & -1.65 \\
\hline 8360 & $\mathrm{~L}$ & -1.60 & 1.85 & -0.174 & 0.304 & -0.62 & 0.46 & 838 & 10.5 & 0.39 & -20.53 & -1.62 \\
\hline 8526 & $\mathrm{~L}$ & -1.48 & 0.29 & -0.087 & 0.064 & -0.51 & 0.75 & & & 0.58 & -20.85 & -1.52 \\
\hline
\end{tabular}




\section{References}

Abraham, R. G., Tanvir, N. R., Santiago, B. X., et al. 1996, MNRAS, 279, L47 (A96)

Allen, C. W. 1973, "Astrophysical Quantities", 3rd Edition, London

Appenzeller, I., Fricke, K. J., Fürtig, W., et al. 1998, The Messenger, 94, 1

Appenzeller, I., Bender, R., Böhm, A., et al., 2000, The Messenger, 100, 44

Babul, A., \& Rees, M. J. 1992, MNRAS, 255, 346

Bahcall, N., Ostriker, J. P., Perlmutter, S., \& Steinhardt, P. J. 1999, Science, 284, 1481

Barden, M., Lehnert, M. D., Tacconi, L., et al. 2003, ApJ, submitted (astro-ph/0302392) (Bar03)

Bell, E. F., Baugh, C. M., Cole, S., Frenk, C. S., \& Lacey, C. G. 2003, MNRAS, 343, 367

Bender, R., Burstein, D., \& Faber, S. M. 1992, ApJ, 399, 462

Bender, R., Appenzeller, I., Böhm, A., et al. 2001, in "Deep Fields", Cristiani, S., Renzini, A., \& Williams, R. E. (eds.), ESO astrophysics symposia, Springer, p. 96

Bershady, M. A. 1997, in "Dark and Visible Matter in Galaxies", Persic, M., \& Salucci, P. (eds.), ASP Conf. Ser. 117, p. 537

Bertin, E., \& Arnouts, S. 1996, A\&AS, 117, 393

Bik, A., Lamers, H. J. G. L. M., Bastian, N., Panagia, N., \& Romanniello, M. 2003, A\&A, 397,473
Binney, J., \& Tremaine, S. 1987, "Galactic Dynamics", Princeton University Press

Böhm, A., Ziegler, B. L., \& Fricke, K. J. 2001, AG Abstract Series Vol. 18, p. 57

Böhm, A., Ziegler, B. L., Fricke, K. J., et al. 2003a, Ap\&SS, 284, 689

Böhm, A., Ziegler, B. L., Saglia, R. P., et al. 2003b, A\&A, submitted (astro-ph/0309263)

Boissier, S., \& Prantzos, N. 2001, MNRAS, 325, 321

Burles, S., \& Tytler, D. 1998, ApJ, 499, 699

Burstein, D., \& Heiles, C. 1982, AJ, 87, 1165

Burstein, D., Bender, R., Faber, S. M., \& Nolthenius, R. 1997, AJ, 114, 1365

Cardelli, J. A., Clayton, G. C., \& Mathis, J. S. 1989, ApJ, 345, 245

Carroll, S. M., \& Press, W. H. 1992, ARA\&A, 30,499

Castillo-Morales, A., \& Schindler, S. 2003, in "Highlights of Spanish Astrophysics III", Gallego, J., Zamorano, J., \& Cardiel, N. (eds.), Kluwer (astro-ph/0303609)

Cole, S., \& Lacey, C. 1996, MNRAS, 281, 716

Combes, F., Boisse, P., Mazure, A., Blanchard, A., \& Seymour, A. 1995, "Galaxies and Cosmology", Springer

Contardo, G., Steinmetz, M., \& Fritzev.Alvensleben, U. 1998, ApJ, 507, 497 
Courteau, S. 1997, AJ, 114, 2402

Dopita, M. A., \& Ryder, S. D. 1994, ApJ, 430, 163

Doroshkevich, A. G., Müller, V., Retzlaff, J., \& Turchaninov, V. 1999, MNRAS, 306, 575

Dressler, A. 1980, ApJ, 236, 351

Dressler, A., Lynden-Bell, D., Burstein, D., et al. 1987, ApJ, 313, 42

Driver, S. P., Warrick, J. C., Phillips, S., \& Windhorst, R. A. 1996, ApJ, 466, L5

Efstathiou, G., \& Silk, J. 1983, Fund. Cosmic Physics, 9, 1

Eggen, O. J., Lynden-Bell, D., \& Sandage, A. R. 1962, 136, 748

Ellis, R. S. 1997, ARA\&A, 35, 389

Elmegreen, B. G. 2003, Ap\&SS, 284, 819

Ferreras, I., \& Silk, J. 2001, ApJ, 557, 165

Freeman, K. C. 1970, ApJ, 160, 811

Frei, Z., \& Gunn, J. E. 1994, AJ, 108, 1476

Frei, Z., Guhathakurta, P., Gunn, J. E., \& Tyson, J. A. 1996, AJ, 111, 174

Fried, J. W., v. Kuhlmann, B., Meisenheimer, K., et al. 2001, A\&A, 367, 788

Fritze-v. Alvensleben, U. 1989, PhD thesis, Universtät Göttingen

Fukugita, M., Shimasaku, K., \& Ichikawa, T. 1995, PASP, 107, 945

Gabasch, A., Bender, R., Hopp, U., et al. 2003, Kennicutt, R. C. 1998, ARA\&A, 36, 189 in prep.

Geller, M. J., \& Huchra J. P. 1989, Science, 246, 879

Giallongo, E., Menci, N., Poli, F., D'Odorico, S., \& Fontana, A. 2000, ApJ, 530, L73
Giovanelli, R., Haynes, M. P., Herter, T., et al. 1997, AJ, 113, 53 (Gio97)

Haynes, M. P., Giovanelli, R., Chamaraux, P., et al. 1999, AJ, 117, 2039 (Ha99)

Heidmann, J., Heidmann, N., \& de Vaucouleurs, G. 1971, Mem. R. Astron. Soc., 75, 85

Heidt, J., Appenzeller, I., Gabasch, A., et al. 2003, A\&A, 398, 49

Hubble, E. 1926, ApJ, 64, 321

Jimenez, R., Padoan, P., Matteucci, F., \& Heavens, A. 1998, MNRAS, 299, 123

Jog, C. J. 1999, ApJ, 522, 661

Jørgensen, I. 1994, PASP, 106, 967

Kajizawa, M. \& Yamada, T. 2001, PASJ, 53, 833

Kannappan, S. J., Barton Gillespie, E., Fabricant, D. G., Franx, M., \& Vogt, N. P. 2002a, proceedings of "Galaxy Evolution: Theory and Observations", Avila-Reese, V., Firmani, C., Frenk, C. S., \& Allen, P. (eds.), RevMexAA, in press (astro-ph/0206088)

Kannappan, S. J., Fabricant, D. G., \& Franx, M. 2002b, AJ, 123, 2358

Karachentsev, I. D., Mitronova, S. N., Karachentseva, V. E., Kudrya, Y. N., \& Jarrett, T. H. 2002, A\&A, 396, 431

Kennicutt, R. C. 1983, ApJ, 272, 54

Kennicutt, R. C. 1992a, ApJS, 79, 255

Kennicutt, R. C. 1992b, ApJ, 388, 310

Kobulnicky, H. A., Willmer, C. N. A., Weiner, B. J., et al. 2003, ApJ, accepted (astro$\mathrm{ph} / 0310347$ )

Koda, J., Sofue, Y., \& Wada, K. 2000a, ApJ, 531, L17 
Koda, J., Sofue, Y., \& Wada, K. 2000b, ApJ, 532,214

Koo, D. C. 2001, in "Deep Fields", Cristiani, S., Renzini, A., \& Williams, R. E. (eds.), ESO astrophysics symposia, Springer, p. 107

Kron, R. G. 1980, ApJS, 43, 305

Lambas, D. G., Tissera, P. B., Sol Alonso, M., \& Coldwell, G. 2002, MNRAS, submitted (astro$\mathrm{ph} / 0212222)$

Longair, M. S. 1998, "Galaxy Formation", Springer

Mao, S., Mo, H. J., \& White, S. D. M. 1998, MNRAS, 297, L71

Marleau, F. R., \& Simard, L. 1998, ApJ, 507, 585 (MS98)

Mathewson, D. S., \& Ford, V. L. 1996, ApJS, 107, 97 (MF96)

McGaugh, S. S., Schombert, J. M., Bothun, G. D., \& de Blok, W. J. G. 2001, ApJ, 533, L99

Mehlert, D., Noll, S., Appenzeller, I., et al. 2002, A\&A, 393, 809

Metcalfe, N., Shanks, T., Campos, A., McCracken, H. J., \& Fong, R. 2001, MNRAS, 323, 779

Milgrom, M. 1983, ApJ, 270, 365

Milvang-Jensen, B. 2003, PhD thesis, University of Nottingham

Milvang-Jensen, B., Aragón-Salamanca, A., Hau, G. K. T., Jørgensen, I., \& Hjorth, J. 2003, MNRAS, 339, L1 (Mil03)

Mo, H. J., Mao, S., \& White, S. D. M. 1998, MNRAS, 295, 319 (MMW98)

Möller, C. S., Fritze-v.Alvensleben, U., Fricke, K. J., \& Calzetti, D. 2001, Ap\&SS, 276, 799
Navarro, J. F., \& Steinmetz, M. 2000, ApJ 538, 477

Noll, S., Mehlert, D., Appenzeller, I., et al. 2003, A\&A, submitted

Osterbrock, D. E. 1993, ApJ, 404, 551

Peacock, J. A. 2003, in "The search for dark matter and dark energy in the universe", from the January 2003 Royal Society Discussion Meeting (astro-ph/0309238)

Peebles, P. J. E. 1993, "Principles of Physical Cosmology", Princeton University Press

Perlmutter, S., Aldering, G., Goldhaber, G., et al. 1999, ApJ, 517, 565

Persic, M., \& Salucci, P. 1991, ApJ, 368, 60 (PS91)

Persic, M., Salucci, P., \& Stel, F. 1996, MNRAS, 281, 27 (Pe96)

Pickles, A. J. 1992, PASP, 110, 863

Pierce, M. J., \& Tully, R. B. 1992, ApJ, 387, 47 (PT92)

Press, W. H., Teukolsky, S. A., Vetterling, W. T., \& Flannery, B. P. 1992, "Numerical Recipes in Fortran 77", Cambridge University Press

Rix, H.-W., Guhathakurta, P., Colless, M., \& Ing, K. 1997, MNRAS, 285, 779

Rubin, V. C., Burstein, D., Ford Jr., W. K., \& Thonnard, N. 1985, ApJ, 289, 81

Rubin, V. C., Waterman, A. H., \& Kenney, J. D. P. 1999, AJ, 118, 236

Ryder, S. D., \& Dopita, M. A. 1994, ApJ, 430, 142

Saha, P. \& Williams, T. B. 1994, AJ, 107, 1295

Sakai, S., Mould, J. R., Hughes, S. M. G., et al. 2000, ApJ, 529, 698 
Sandage, A. 1986, A\&A, 161, 89

Sellwood, J. A., \& Kosowsky, A. 2001, in "Gas and Galaxy Evolution", ASP Conf. Proceedings, Vol. 240, Hibbard, E., Rupen, M. \& van Gorkum, J. H. (eds.), Astronomical Society of the Pacific, p. 311

Shane, N. S., \& James, P. A. 2002, in "Disks of Galaxies: Kinematics, Dynamics and Perturbations", Athanassoula, E., Bosma, A., \& Mujica, R. (eds.), ASP Conf. Proceedings, Vol. 275, p. 323

Silk, J. 2000, MNRAS, 324, 313

Simard, L., \& Pritchet, C. J. 1998, ApJ, 505, 96

Simard, L., \& Pritchet, C. J. 1999, PASP, 111, 453 (SP99)

Simard, L., Willmer, C. N., Vogt, N. P., et al. 2002, ApJS, 142, 1

Sinachopoulos, D., \& van Dessel, E. 1998, A\&AS, 130, 290

Sofue, Y., \& Rubin, V. 2001, ARA\&A, 39, 137

Spergel, D. N., Verde, L., Peiris, H. V., et al. 2003, ApJS, 148, 175

Steinmetz, M., \& Navarro, J. F. 1999, ApJ, 513, 555

Struck-Marcell, C., \& Tinsley, B. M. 1978, ApJ, 221,562

Swinbank, A. M., Smith, J., Bower, R. G., et al. 2003, ApJ, submitted (astro-ph/0307521)

Tegmark, M., Zaldarriaga, M., \& Hamilton, J. S. 2001, in "Sources and Detection of Dark Matter and Dark Energy in the Universe", Cline, D. B. (ed.), Springer, p. 128

Tully, R. B., \& Fisher, J. R. 1977, A\&A, 54, 661

Tully, R. B., \& Fouqué, P. 1985, ApJS, 58, 67 (TF85)
Tully, R. B., Pierce, M. J., Huang, J.-S., et al. 1998, AJ, 115, 2264 (Tu98)

Tully, R. B., \& Pierce, M. J. 2000, ApJ, 533, 744 (TP00)

van den Bergh, S., Abraham, R. G., Ellis, R. S., Tanvir, R. S., Santiago, B. X., \& Glazebrook, K. G. 1996, AJ, 112, 359 (vdB96)

van den Bergh, S. 2002, PASP, 114, 797

van den Bosch, F. C. 2003, in "The Mass of Galaxies at Low and High Redshift", proceedings of the ESO Workshop held in Venice, Italy 24-26 October 2001, p. 250

Verheijen, M. A. W. 2001, ApJ, 563, 694

Vogt, N. P. 2000, in "Dynamics of Galaxies: from the Early Universe to the Present", ASP Conf. Ser. 197, Combes, F., Mamon, G. A., \& Charmandrais, V. (eds.), p. 435

Vogt, N. P. 2001, in "Deep Fields", Cristiani, S., Renzini, A., \& Williams, R. E. (eds.), ESO astrophysics symposia, Springer, p. 112

Vogt, N. P., Forbes, D. A., Phillips, A. C., et al. 1996, ApJ, 465, L15

Vogt, N. P., Phillips, A. C., Faber, S. M., et al. 1997, ApJ, 479, L121

Wagner, R. M., in "Astronomical CCD observing and reduction techniques", ASP Conf. Series Vol. 23, Howell., S. B. (ed.), Astronomical Society of the Pacific, 1992, p. 160

Williams, R. E., Blacker, B., Dickinson, M., et al. 1996, AJ, 112, 1335

Williams, R. E., Baum, S., Bergeron, L., et al. 2000, AJ, 120, 2735

Ziegler, B. L., Bower, R. G., Smail, I., Davies, R. L., \& Lee, D. 2001, MNRAS, 325, 1571

Ziegler, B. L., Böhm, A., Fricke, K. J., et al. 2002, ApJ, 564, L69 
Ziegler, B. L., Thomas, D., Böhm, A., et al. 2003, A\&A, in prep. 


\section{Acknowledgements}

At first, I want to thank Prof. Dr. K. J. Fricke for his continous support and for giving me the opportunity to acquire the doctoral level in a spellbinding field of modern extragalactic astronomy with data from a "state-of-the-art" telescope, the VLT; last but not least for showing confidence in my scientific abilities on so many occasions. My further thanks go to Prof. Dr. F. Kneer, who kindly agreed to become the secondary referee of my thesis. I am also very grateful to Dr. B. L. Ziegler, the leader of our Junior Research Group (sponsored by the Volkswagen Foundation) for his support and encouragement, many helpful and interesting discussions, and also for the thorough proof-reading of the draft and many useful comments on it.

Furthermore, I wish to thank the other members of the disputation committee, Prof. Dr. A. Bahr, Prof. Dr. U. Christensen, Prof. Dr. S. Dreizler and Prof. Dr. W. Kollatschny.

Thanks also to Dr. I. Berentzen, Priv. Doz. Dr. U. Fritze-von Alvensleben, Dr. K. Jäger and Dr. P. Papaderos for their proof-reading of some chapters.

I am also grateful to the P.I. of the FORS Deep Field project, Prof. Dr. I. Appenzeller, for his continous support of this project.

Thanks a lot to the members of our Junior Research Group, Dr. I. Berentzen, A. Fritz, B. Gerken and Dr. K. Jäger, for all helpful and interesting discussions, many helps and hints and the nice working atmosphere.

I am also grateful to Drs. J. Heidt, D. Mehlert and S. Noll (all Heidelberg), who performed the main parts of the spectroscopy for this project during the Garantueed Time Observations in 2000 and 2001. I also wish to thank Drs. K. Reinsch and C. Möllenhoff (Heidelberg) for the performance of the pilot observation in December 1999.

Other people from foreign institutes who contributed to my understanding of the project in several ways and whom I want to thank are Prof. Dr. R. Bender (München) and Drs. I. Ferreras (Oxford), U. Hopp (München), S. Kannappan (Cambridge, USA), B. MilvangJensen (Garching), R. P. Saglia (München), P. Salucci (Trieste) and M. A. W. Verheijen (Potsdam).

A very big help during the course of my main study period has been the support of my parents, my grandmothers and my grandaunt, and I'm deeply thankful to them all. Thanks also to my sister Verena, my brother-in-law to be, Gernot, and my cousin Dagmar for their love and many chats which were important to me.

Last but not least, thanks to Bernhard, Christian, Jan-Hauke and Michael, for enriching my life with their friendship and for having cheered me up so many times.

I am furthermore grateful for the financial support by the Volkswagen Foundation.

This thesis has been created on computers of the Universitätssternwarte Göttingen and the Gesellschaft für Wissenschaftliche Datenverarbeitung Göttingen (GWDG). 


\section{Lebenslauf}

Asmus Böhm

Lange-Geismar-Str. 33

37073 Göttingen

Geburtsdatum: 21.09.1969

Geburtsort: Kassel

Staatsangehörigkeit: deutsch

Familienstand: ledig

Seit 12/1999 Wissenschaftlicher Mitarbeiter an der Universitätssternwarte Göttingen; Mitwirkung u.a. am ,FORS Deep Field“-Projekt und der spektrophotometrischen und kinematischen Analyse von Haufen-Galaxien; Vorträge auf Tagungen z.B. in München, Kiel, Berlin;

Betreuung des Physik-Anfängerpraktikums

07/1999 Beginn der Doktorarbeit an der Universitätssternwarte Göttingen unter der Betreuung von Prof. Dr. K. J. Fricke mit dem Thema ,,The Evolution of Distant Spiral Galaxies in the FORS Deep Field“

05/1999 Studienabschluss an der Universität Göttingen:

Diplom in Physik mit der Gesamtnote ,,Sehr gut“

11/1997 Beginn der Diplomarbeit an der Universitätssternwarte Göttingen unter der Betreuung von Prof. Dr. K. J. Fricke mit dem Thema „,Analyse von Quasarumgebungen im niedrigen Rotverschiebungsbereich anhand von Direktbildern, Multiobjektspektren und Datenbanken“

10/1992 Vordiplom Physik

10/1990 Aufnahme des Physikstudiums in Göttingen

06/1988 Allgemeine Hochschulreife am Geschwister-Scholl-Gymnasium Melsungen

1985 bis 1988 Geschwister-Scholl-Gymnasium Melsungen

1978 bis 1985 Integrierte Gesamtschule Guxhagen

1974 bis 1978 Grundschule Guxhagen 